\title{
Altersspezifische Anpassungen an ein Dehntraining
}

\author{
Dissertation \\ zur Erlangung des akademischen Grades eines \\ Doktors der Philosophie \\ der Fakultät HW \\ Bereich Empirische Humanwissenschaften \\ der Universität des Saarlandes
}

\author{
vorgelegt von \\ Thomas Haab \\ aus Wiesbaden
}

Saarbrücken, 2017 


\section{Dekan:}

Univ.-Prof. Dr. Cornelius König

\section{Berichterstatter:}

Univ.-Prof. Dr. Georg Wydra

Univ.-Prof. Dr. Michael Fröhlich

\section{Tag der Disputation:}

21.11.2017 


\section{Danksagung}

Mein größter Dank gilt meinem Doktorvater, Univ.-Prof. Dr. Georg Wydra, der mir dieses Promotionsprojekt ermöglicht hat. Vielen Dank für das Vertrauen und die vielfältige Unterstützung in all den Jahren. Ebenso bedanke ich mich herzlich für die wissenschaftliche und methodische Betreuung mit wichtigen Denkanstößen sowie den nötigen Freiraum, um dieses Projekt zu realisieren.

Mein nächster Dank gilt Univ.-Prof. Dr. Michael Fröhlich für die methodische Unterstützung und Betreuung von trainingswissenschaftlicher Seite. Herzlichen Dank für die netten Gespräche und vielen Ratschläge.

Ich danke ganz besonders meinem Kollegen vom Sportwissenschaftlichen Institut der Universität des Saarlandes und guten Freund, Christian Kaczmarek, für seine großartige Unterstützung und Hilfsbereitschaft in allen Lebenslagen. Vielen Dank für die sensationelle Zeit im Saarland! Auch meinen anderen lieben Kolleginnen und Kollegen danke ich sehr herzlich für die vielfältige Unterstützung: Andrea Dincher, Christina Lutz, Christoph Engert, Claudia Silberberg, Dr. Franz Marschall, Dr. Markus Schwarz, Dr. Oliver Ludwig, Matthias Massing und Olaf Karthein.

Vielen Dank an alle Probanden, Studentinnen und Studenten, die an diesem Promotionsprojekt mitgewirkt haben.

Nicht zuletzt danke ich meiner Familie und meinen Freunden für ihr Verständnis, dass ich in den letzten Jahren nicht viel Zeit hatte.

Ein ganz besonderer Dank gilt meiner Freundin Alissa Steinmetz für ihre unglaubliche Unterstützung in den letzten Jahren. Ich freue mich jetzt darauf mehr Zeit mit Dir verbringen zu können. Ich hätte es ohne Dich nicht geschafft! 


\section{Inhaltsverzeichnis}

VORWORT 1

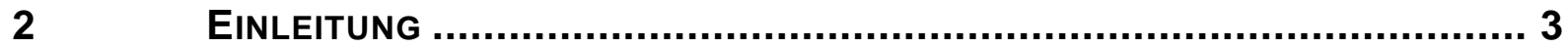

2.1 Grundlagen eines Dehntrainings.................................................... 4

2.1.1 Dehntraining und Beweglichkeit ..................................................................

2.1.2 Gestaltung eines Dehntrainings................................................................

2.2 Einflussfaktoren auf die Beweglichkeit .......................................... 11

2.2.1 Anatomische Einflussfaktoren .....................................................................14

2.2.2 Neurophysiologische Einflussfaktoren ..................................................21

2.2.3 Lebensalter ..........................................................................................

2.3 Biomechanische Grundlagen ....................................................... 32

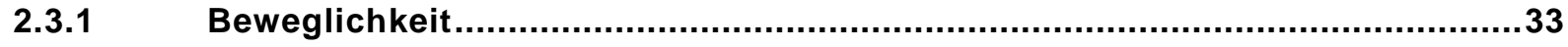

2.3.2 Dehnungsspannung..............................................................................

2.3.3 Viskoelastische Eigenschaften ...................................................................37

2.3.4 Stiffness ................................................................................................

2.3.5 Fläche unterhalb der Dehnungsspannungskurve .........................................42

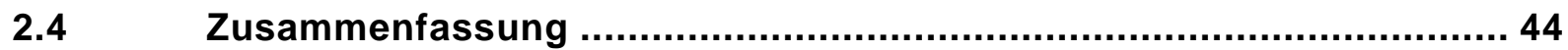

3 Altersspezifische Anpassungen an ein Dehntraining: Ein

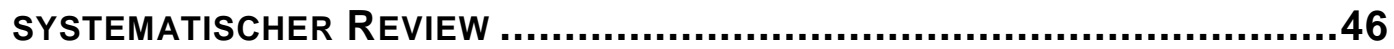

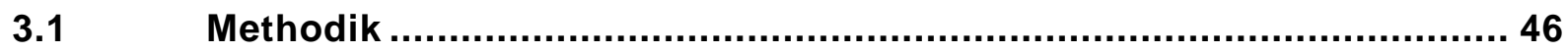

3.1.1 Abhängige Variablen ............................................................................

3.1.2 Bewertung der Primärstudien ...............................................................50

3.1.3 Datenextraktion ...................................................................................

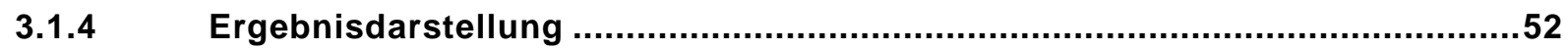

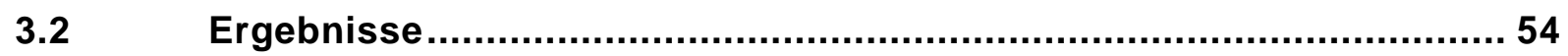

3.2.1 Methodologische Qualität der Primärstudien …………..............................57

3.2.2 Darstellung der Primärstudien ..................................................................59

3.2.3 Maximale Beweglichkeit ................................................................................

3.2.4 Maximale Dehnungsspannung ……………................................................86

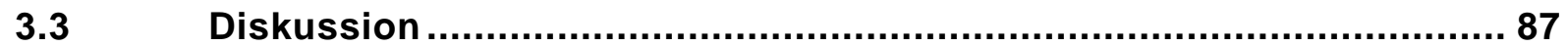

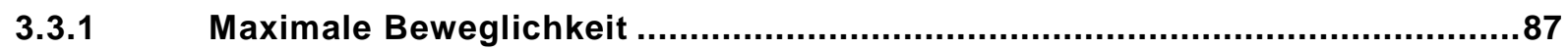




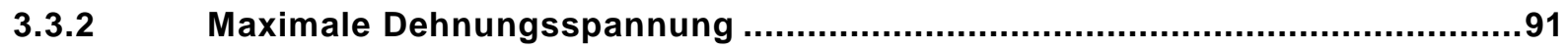

3.3.3 Subanalyse weiterer biomechanischer Parameter .......................................91

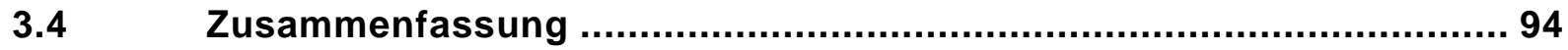

4 Altersspezifische Anpassungen an ein Dehntraining: Eine EMPIRISCHE UNTERSUCHUNG ...................................................96

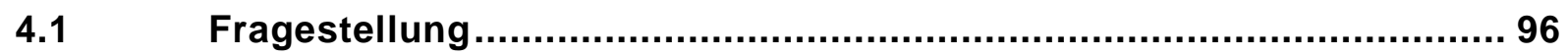

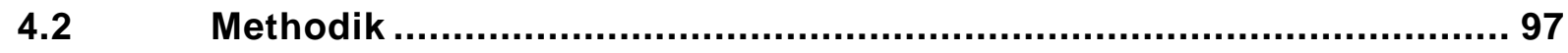

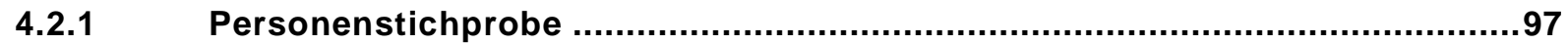

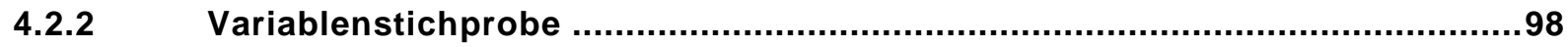

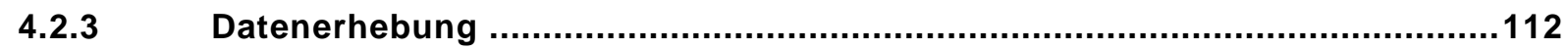

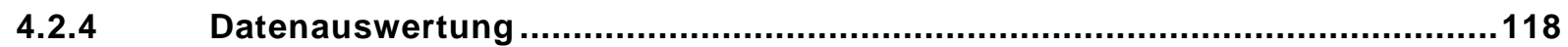

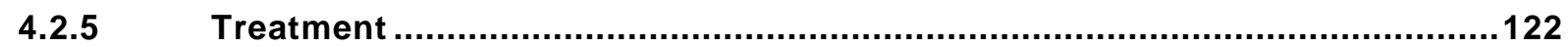

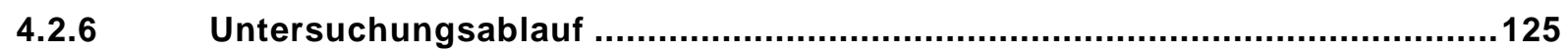

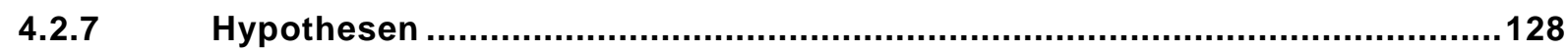

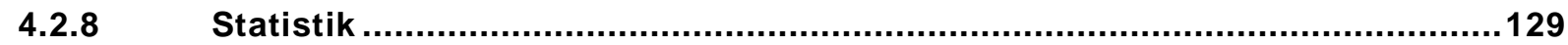

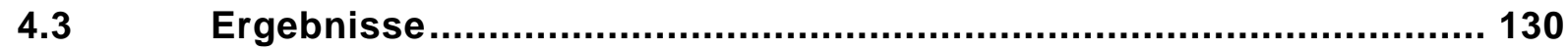

4.3.1 Biomechanische Parameter..................................................................... 132

4.3.2 Neurophysiologische Parameter ................................................................134

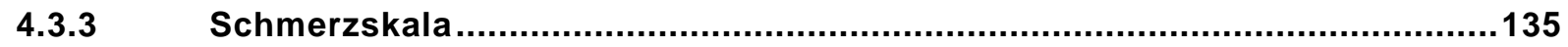

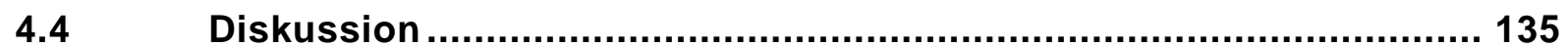

4.4.1 Biomechanische Parameter...................................................................136

4.4.2 Neurophysiologische Parameter ................................................................139

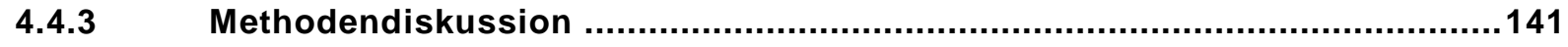

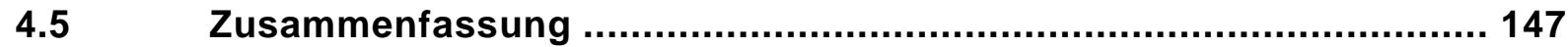

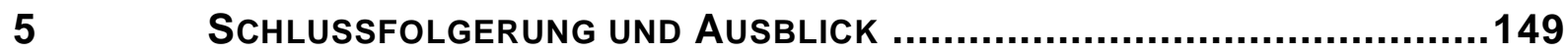

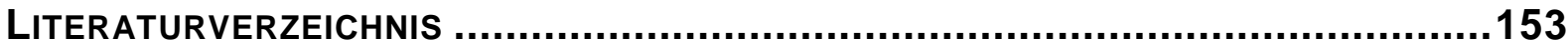

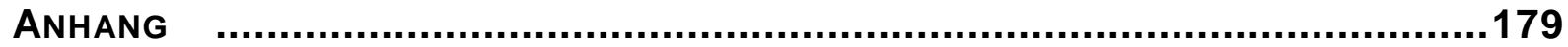




\section{Abbildungsverzeichnis}

Abb. 1 Differenzierung der verschiedenen Formen der Muskeldehnung …....................

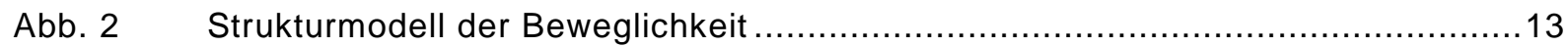

Abb. 3 Darstellung verschiedener Gelenktypen ....................................................... 15

Abb. $4 \quad$ Ultrastrukturelle Darstellung einer Muskelfaser ............................................16

Abb. 5 Das Drei-Komponenten-Modell mit den seriell-elastischen, den parallelelastischen und den kontraktilen Komponenten ........................................17

Abb. 6 Schematische Darstellung eines Sarkomers mit Myosin-, Aktin- und Titinfilament

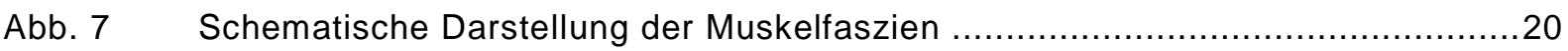

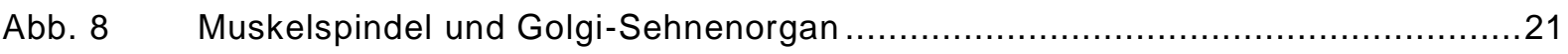

Abb. 9 Lage der Muskelspindeln und Golgi-Sehnenorgane (oben) und Entladungsmuster bei Beanspruchung der Muskulatur (unten) ................................................24

Abb. 10 Schematische Darstellung einer Oberflächenelektromyographie ......................25

Abb. 11 Veränderung der Beweglichkeit im Alternsgang am Beispiel des Finger-BodenAbstandes 29

Abb. 12 Exemplarische Darstellung einer Dehnungsspannungskurve .............................35

Abb. 13 Exemplarische Dehnungsspannungskurve mit Darstellung der elastischen und plastischen Region sowie dem Yield-Point ................................................. 36

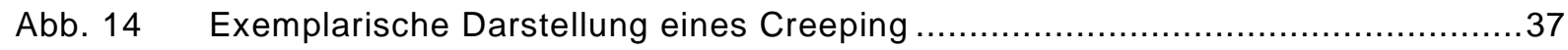

Abb. 15 Exemplarische Darstellung einer Spannungsrelaxation ...................................

Abb. 16 Exemplarische Darstellung einer Hystereseschleife ..........................................40

Abb. 17 Exemplarische Darstellung von zwei Spannungskurven bei hoher Dehnungsgeschwindigkeit (links) und bei geringer Dehnungsgeschwindigkeit (rechts)

Abb. 18 Exemplarische Darstellung der Fläche unterhalb der Dehnungsspannungskurve 43

Abb. 19 Fließdiagramm zur Literaturrecherche ...................................................55

Abb. 20 Apparativer Straight-Leg-Raise-Test (ASLRT) …..........................................99

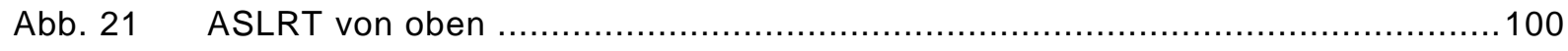

Abb. 22 Schematische Darstellung einer Longitudinalverschiebung während einer Dehnung auf dem ASLRT .................................................................. 102

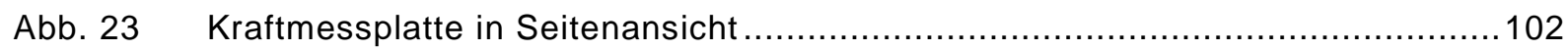

Abb. 24 Auf dem Hebelarm montierte Kraftmessplatte in Vorderansicht ........................104

Abb. 25 Sprunggelenksbandage mit eingenähtem Klettband an der Rückseite ...............105 
Abb. 26 Beispiel einer Dehnungsspannungskurve während einer Muskeldehnung mit Darstellung der abhängigen Variablen .............................................. 108

Abb. 27 Beispielhafte Darstellung der EMG-Parameter eines Probanden ...................110

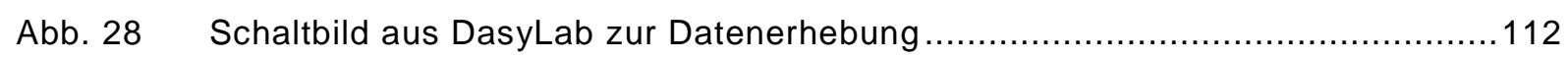

Abb. 29 Schaltbild aus DasyLab zur Weiterverarbeitung der Rohdaten aus der

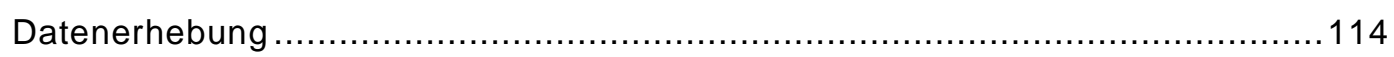

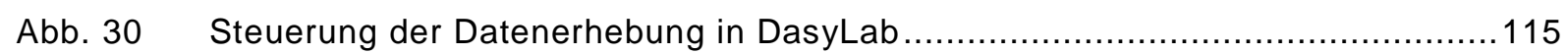

Abb. 31 Proband auf ASLRT mit EMG-Ableitungen an M. biceps femoris .................118

Abb. 32 Beispielhafte Darstellung eines gleichgerichteten EMG-Signals in MatLab eines Probanden zur Erfassung des EMG-Onset..................................... 122

Abb. 33 Schriftliche Anweisung des Dehntrainings für die Probanden ......................123

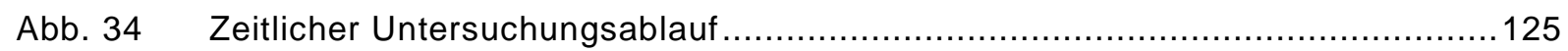

Abb. 35 Prozentuale Veränderung der biomechanischen Parameter zwischen Pre- und

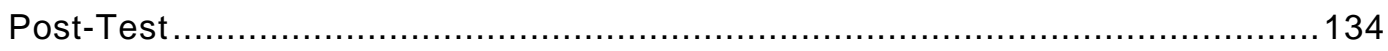

Abb. 36 Prozentuale Veränderung der neurophysiologischen Parameter zwischen Pre- und

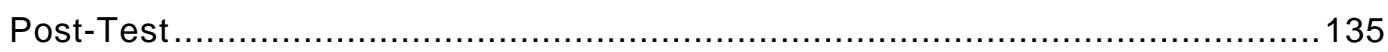




\section{Tabellenverzeichnis}

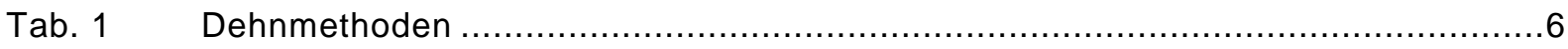

Tab. 2 Filter in den verwendeten Datenbanken ................................................... 47

Tab. 3 Deskriptoren zur Literaturrecherche ........................................................... 48

Tab. $4 \quad$ Kodierung und Studiendetails der Primärstudien ..........................................52

Tab. 5 Methodologische Beurteilung der Primärstudien mit der modifizierten PEDro-Skala

Tab. 6 Darstellung von Studiendetails und Effektstärken bei der maximalen Beweglichkeit der Kniebeugemuskulatur

Tab. 7 Darstellung von Studiendetails und Effektstärken bei der maximalen Beweglichkeit der Kniestreckmuskulatur .74

Tab. 8 Darstellung von Studiendetails und Effektstärken bei der maximalen Beweglichkeit der Hüftbeugemuskulatur .75

Tab. 9 Darstellung von Studiendetails und Effektstärken bei der maximalen Beweglichkeit der Wadenmuskulatur

Tab. 10 Darstellung von Studiendetails und Effektstärken bei der maximalen Dehnungsspannung der Kniebeugemuskulatur

Tab. 11 Darstellung von Studiendetails und Effektstärken bei der maximalen Dehnungsspannung der Wadenmuskulatur

Tab. 12 Ergebnisse der ANOVA und Darstellung der Effektstärken durch die Veränderung der ROM in Abhängigkeit von Merkmalen eines Dehntrainings .86

Tab. 13 Reliabilitätskoeffizienten der Dehnungsspannung unterteilt in die beiden

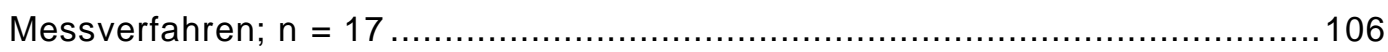

Tab. 14 Beschreibung der abhängigen Variablen ................................................... 107

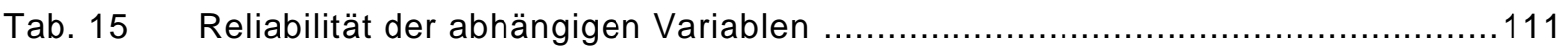

Tab. 16 Ergebnisse der ANOVA und Messwerte zu Pre- und Post-Test ......................131 


\section{Abkürzungsverzeichnis}

\begin{tabular}{|c|c|}
\hline ANOVA & Varianzanalyse \\
\hline ASLRT & Apparativer Straight-Leg-Raise-Test \\
\hline $\mathrm{cm}$ & Zentimeter \\
\hline EMG & Elektromyographie \\
\hline ES & Effektstärke \\
\hline $\mathrm{Hz}$ & Hertz \\
\hline ICC & Intraklassenkorrelation \\
\hline $\mathrm{kg}$ & Kilogramm \\
\hline $\mathrm{mm}$ & Millimeter \\
\hline $\mathrm{ms}$ & Millisekunde \\
\hline MTU & Muscle Tendon Unit \\
\hline $\mathrm{N}$ & Newton \\
\hline $\mathrm{Nm}$ & Newtonmeter \\
\hline OEMG & Oberflächenelektromyographie \\
\hline PEE & Passive-elastische Energie \\
\hline PES & Passive-elastische Stiffness \\
\hline PNF & Propriozeptive Neuromuskuläre Fazilitation \\
\hline ROM & Range of Motion \\
\hline sec. & Sekunde \\
\hline $\mathrm{U} / \mathrm{min}$ & Umdrehungen pro Minute \\
\hline $\mathrm{W} / \mathrm{kg} \mathrm{KG}$ & Watt pro Kilogramm Körpergewicht \\
\hline$\circ$ & Winkelgrad \\
\hline${ }^{\circ} \mathrm{C}$ & Grad Celsius \\
\hline
\end{tabular}




\section{Vorwort}

Die vorliegende Arbeit beschäftigt sich mit altersspezifischen Anpassungserscheinungen an ein Dehntraining. Hierfür wurde eine Metaanalyse und eine empirische Untersuchung durchgeführt. Die methodische Vorgehensweise sowie zentrale Ergebnisse dieser Untersuchungen wurden als wissenschaftliche Beiträge auf Kongressen vorgestellt oder in Fachzeitschriften publiziert. Es soll nachfolgend erläutert werden, welche Inhalte der hier vorliegenden Arbeit als wissenschaftliche Beiträge publiziert wurden und deshalb in den Formulierungen in den Publikationen ganz oder teilweise identisch sein können. Auf die entsprechenden Publikationen wird im Verlauf dieser Arbeit an gegebener Stelle hingewiesen.

Im dritten Kapitel erfolgt die Aufarbeitung des aktuellen Forschungsstandes im Rahmen einer systematischen Literaturrecherche und einer metaanalytischen Betrachtung. Diese Untersuchung wurde im German Journal of Exercise and Sport Research im Juli 2017 veröffentlicht:

Haab, T., Martini, J., Baluktsian, S., \& Wydra, G. (2017). Altersspezifische Anpassungen an ein Dehntraining. Eine metaanalytische Betrachtung. German Journal of Exercise and Sport Research. doi: 10.1007/s12662-017-0464-1

Im vierten Kapitel erfolgt die Darstellung einer empirischen Untersuchung. Hier wird eine Voruntersuchung dargestellt, die im Rahmen einer Evaluierung eines Messsystems notwendig war. Diese Voruntersuchung wurde als Poster auf dem 22. Sportwissenschaftlichen Hochschultag der Deutschen Vereinigung für Sportwissenschaft vorgestellt. Der Abstract zum Poster wurde im Oktober 2015 veröffentlicht:

Haab, T., Schmid, S., Sahner, D., Fröhlich, M., Ludwig, O., \& Wydra, G. (2015). Ausgleich einer Longitudinalverschiebung des Beines an einem apparativen Straight-Leg-Raise-Test. In T. Könecke, H. Preuß, \& W.I. Schöllhorn (Hrsg.), 
Moving Minds - Crossing Boundaries in Sport Science (S. 326). Hamburg: Feldhaus.

Im Rahmen der empirischen Untersuchung wurden erste Ergebnisse als Vortrag auf der 10. Jahrestagung der Deutschen Gesellschaft für Biomechanik vorgestellt. Der Abstract zum Vortrag wurde im März 2017 veröffentlicht:

Haab, T., De Borger, S., \& Wydra, G. (2017). Altersspezifische Unterschiede nach einem zehnwöchigen Dehntraining. In DGfB (Hrsg.), 10. Jahrestagung der Deutschen Gesellschaft für Biomechanik (DGfB). Abstractband. (S. 168). Hannover.

Die Operationalisierung der Anpassungserscheinungen in der empirischen Untersuchung erfolgte durch biomechanische und neurophysiologische Parameter. Die Untersuchung mit dem Schwerpunkt auf den biomechanischen Parametern wurde im Journal of Physical Therapy Science im Juni 2017 veröffentlicht:

Haab, T., \& Wydra, G. (2017). The effect of age on hamstring passive properties after a 10-week stretch training. Journal of Physical Therapy Science, 29(6), 1048-1053. doi: 10.1589/jpts.29.1048

Die Untersuchung zu den neurophysiologischen Parametern wurde im Journal of Exercise Physiology Online im August 2017 veröffentlicht:

Haab, T., Massing, M., \& Wydra, G. (2017). EMG onset after a 10 week stretching intervention. A comparison between older and younger adults. Journal of Exercise Physiology Online, 20(4), 121-131. 


\section{Einleitung}

Das Dehnen der Muskulatur ist ein weitverbreiteter Bestandteil im Sport, im rehabilitativen als auch im gesundheitlichen Kontext. Aus sportlicher Perspektive wird eine Dehnung unter anderem zur Verbesserung der Bewegungsreichweite im Rahmen eines Aufwärmprogramms durchgeführt (Smith, 1994; Young \& Behm, 2002; Young, 2007). Des Weiteren wird ein Dehntraining als Präventionsmaßnahme zur Vermeidung von Verletzungen diskutiert (Schneider, Schmitt, Zalewski, \& Gantz, 2011; Witvrouw, Mahieu, Danneels, \& McNair, 2004). Aus gesundheitlicher Perspektive kommt eine Muskeldehnung zur Verringerung von Muskelverspannungen im therapeutischen Kontext zum Einsatz (Carlson \& Curran, 1994; Carlson, Collins, Nitz, Sturgis, \& Rogers, 1990) sowie zur Behandlung und Vorbeugung von Muskelkrämpfen (Bertolasi, Grandis, Bongiovanni, Zanette, \& Gasperini, 1993; Edouard, 2014; Miller, Stone, Huxel, \& Edwards, 2010) und zur Behandlung von Rückenschmerzen (Han, Choi, \& Shin, 2016; Van Tulder, Malmivaara, Esmail, \& Koes, 2000).

Die Beweglichkeit stellt bei Personen im höheren Lebensalter eine wichtige Ressource dar, um eine adäquate Alltagsmotorik zu gewährleisten (Farage, Miller, Ajayi, \& Hutchins, 2012). Aus diesem Grund wird ein Dehntraining zur Verbesserung der Beweglichkeit empfohlen (Chodzko-Zajko et al., 2009). Ältere Personen weisen eine verringerte Beweglichkeit auf (Gajdosik, Vander Linden, \& Williams, 1999; Nonaka et al., 2002). Dennoch zeigen sie kurzfristig nach einer Dehnungsintervention ähnliche Zuwachsraten hinsichtlich der maximalen Beweglichkeit wie eine jüngere Vergleichsgruppe (Ryan, Herda, Costa, Herda, \& Cramer, 2014). Weiterhin konnte Wydra (2008) eine Abhängigkeit der Beweglichkeit vom Lebensalter zeigen und, dass die Trainierbarkeit altersunabhängig zu sein scheint. Allerdings wurde bisher bei älteren Personen die Trainierbarkeit der Muskulatur hinsichtlich der Muskeldehnfähigkeit und der Beweglichkeit über einen längeren Zeitraum kaum untersucht. Aus diesem Grund wird in Kapitel 4 eine empirische Untersuchung zu altersspezifischen Anpassungserscheinungen durch ein langzeitiges Dehntraining vorgestellt. Zuvor wird in Kapitel 3 der aktuelle Forschungsstand zu Anpassungserscheinun- 
gen an ein Dehntraining im Rahmen einer systematischen Literaturrecherche aufgearbeitet und einer metaanalytischen Betrachtung unterzogen. In diesem einleitenden Kapitel erfolgt zunächst eine Einführung in die Thematik des Dehntrainings. In Kapitel 2.2.3 wird explizit auf die Veränderung der Beweglichkeit im Alter eingegangen.

\subsection{Grundlagen eines Dehntrainings}

Bevor die Grundlagen eines Dehntrainings erläutert werden, ist eine Begriffsbestimmung notwendig, um die Begriffe Dehntraining und Beweglichkeitstraining voneinander abzugrenzen.

Eine Muskeldehnung wird nach Olivier, Marschall und Büsch (2008, S. 233) definiert als eine „... mechanische Zugbelastung, die als Entfernung von Muskelursprung und -ansatz beschrieben werden kann. Die dadurch insbesondere im Bindegewebe ausgelösten Adaptationen tragen zur Verbesserung der $\mathrm{Be}$ weglichkeit bei“. Wird durch ein Dehntraining das Ziel verfolgt die Beweglichkeit zu verbessern, so wird manchmal fälschlicherweise der Begriff Beweglichkeitstraining verwendet. Kisner und Colby (2012, S. 91) beschreiben den Unterschied zwischen Dehntraining (stretching) und einem Beweglichkeitstraining (ROM exercise) wie folgt: "Stretching takes soft tissue structures beyond their available length to increase ROM. ROM exercises stay within the limits of tissue extensibility to maintain the available length of tissues". In der vorliegenden Arbeit liegt der Schwerpunkt auf den Anpassungserscheinungen durch ein Dehntraining. Die Verbesserung der Beweglichkeit wird dabei eine durchaus relevante Rolle spielen. Dennoch werden die Anpassungserscheinungen auch auf muskulärer Ebene im Sinne einer Muskeldehnfähigkeit thematisiert. Ausgehend von der oben aufgeführten Definition von Kisner und Colby (2012, S. 91) wird in dieser Arbeit der Begriff des Dehntrainings verwendet. 


\subsubsection{Dehntraining und Beweglichkeit}

Das primäre Ziel eines Dehntrainings besteht zumeist in einer Erweiterung der Beweglichkeit (Range of Motion = ROM). Im deutschsprachigen Raum beschreibt die Beweglichkeit allgemein nach Freiwald $(2009$, S. 18) „... die maximal mögliche Amplitude in einem Gelenk". Nach Olivier, Marschall und Büsch (2008, S. 230) ist die Beweglichkeit bezogen auf sportliche Aktivitäten „... eine trainierbare zusammengesetzte Einflussgröße sportlicher Bewegungen. Sie beschreibt den Teilaspekt der sportlichen Leistungsfähigkeit, sportliche Bewegungen mit optimaler Reichweite ausführen zu können“. Die Beweglichkeit beruht unter anderem auch auf der muskulären Dehnbarkeit. Diesen Aspekt greift Thienes (2000, S. 33) unter folgender Definition mit auf: „Beweglichkeit ist die Fähigkeit, auf der Grundlage muskulärer Dehnbarkeit eine durch die jeweilige Gelenkstruktur vorgegebene Amplitude innerhalb eines Bewegungsablaufes auszuschöpfen sowie Körperhaltungen in maximalen Winkelstellungen der beteiligten Gelenke einnehmen zu können". Im englischsprachigen Raum wird bezugnehmend auf den deutschen Begriff "Beweglichkeit“ eine Zusammensetzung der Begriffe "flexion" und "capability" verwendet: Flexibility (Alter, 2004; de Araújo, 2004). Auch im englischsprachigen Raum wird die Flexibility auf eine maximal zu erreichende Beweglichkeit definiert: „The maximum physiological passive range of motion of a given joint movement" (de Araújo, 2004, S. 5). Die Bedeutung eines Dehntrainings und der hiermit verbundenen Verbesserung der Beweglichkeit verdeutlicht das American College of Sports Medicine, das sowohl für jüngere (Pollock et al., 1998) als auch für ältere Erwachsene (Garber et al., 2011) ein Dehntraining ausdrücklich empfiehlt. Insbesondere der Einfluss einer guten Beweglichkeit in der Alltagsmotorik wird von Klee und Wiemann (2005, S. 12) hervorgehoben. Hierbei beschreiben sie die Bedeutung der Beweglichkeit hinsichtlich der Altagstätigkeiten wie zum Beispiel Ankleiden, Körperpflege oder Haus- und Gartenarbeit sowie in manchen alltäglichen Situationen die Notwendigkeit, Hindernissen auszuweichen oder Gefahrensituationen zu umgehen. Des Weiteren kann aufgrund einer guten Beweglichkeit das Gefühl einer guten körperlichen Fitness gefördert werden (ebd., S. 13). 


\subsubsection{Gestaltung eines Dehntrainings}

Die Gestaltung eines Dehntrainings hängt zum einen von der Auswahl der Dehnmethode und zum anderen von der Gestaltung der Belastungsparameter ab. Im Folgenden werden die unterschiedlichen Dehnmethoden beschrieben und anschließend wird auf die Belastungsparameter eingegangen.

Die Dehnmethoden lassen sich in statische und dynamische Grundmethoden sowie in die "Propriozeptive Neuromuskuläre Fazilitation“ (PNF-Methoden) unterteilen (Alter, 2004, S. 157 -181). Die PNF-Methode nutzt neuromuskuläre Mechanismen durch die Stimulation von Propriozeptoren (Knott \& Voss, 1968, S. 4). Das Ziel der verschiedenen Techniken der PNF-Methode ist „... die funktionelle Bewegung durch Fazilitation, Inhibition, Kräftigung und Entspannung von Muskelgruppen zu fördern" (Beckers, 2013, S. 36). Da in der Literatur eine Vielzahl an unterschiedlichen Dehnmethoden existiert, hat Klee (2003, S. 127) die fünf gebräuchlichsten Dehnmethoden zusammengefasst (Tab. 1).

Tab. 1 Dehnmethoden (mod. nach Klee, 2003, S. 127)

\begin{tabular}{|c|c|}
\hline \multicolumn{1}{|c|}{ Dehnmethode } & \multicolumn{1}{c|}{ Beschreibung } \\
\hline Dynamisches Dehnen & Gleichmäßiges Einnehmen und Lösen der Dehnposition \\
\hline Statisches Dehnen & Einnehmen und Halten der Dehnposition \\
\hline PNF: AC-Dehnen & $\begin{array}{l}\text { Antagonist-Contract; der Muskel wird durch Kontraktion der an- } \\
\text { tagonistischen Muskulatur statisch gedehnt }\end{array}$ \\
\hline PNF: CR-Dehnen & $\begin{array}{l}\text { Contract-Relax; der Muskel wird vor der Dehnung isometrisch } \\
\text { kontrahiert und dann passiv in die Dehnposition geführt }\end{array}$ \\
\hline PNF: CR-AC-Dehnen & $\begin{array}{l}\text { Contract-Relax-Antagonist-Contract; der Muskel wird vor der } \\
\text { Dehnung isometrisch kontrahiert und dann durch Kontraktion der } \\
\text { antagonistischen Muskulatur in die Dehnposition geführt }\end{array}$ \\
\hline
\end{tabular}

Neben den oben genannten Dehnmethoden findet sich in der Literatur zudem oftmals der Zusatz der aktiven und passiven Dehnung (Schnabel, 2005, S. 281; Thienes, 2000, S. 85; Weineck, 2007, S. 749). Ein aktives Dehnen bedeutet demnach, dass durch Kontrahieren des Antagonisten die zu dehnende Musku- 
latur in die Endstellung gebracht wird. Ein passives Dehnen bedeutet, dass beispielsweise durch Partnerhilfe ohne Aktivierung des Antagonisten die zu dehnende Muskulatur passiv in die Endstellung gebracht wird. Da diese zusätzlichen Begriffe in Anlehnung an Harre (1975, S. 172) missverständlich sind, schlagen Wydra, Glück und Roemer (1999, S. 10) stattdessen die Begriffe Eigen- und Fremddehnung vor. Diese Begriffe, die die Dehnungsart präzisieren, gehen auf handlungstheoretische Überlegungen zurück (ebd.). Bei einer Eigendehnung dehnt sich die Person selbst und bei einer Fremddehnung wird die Person gedehnt (Abb. 1). Die Autoren führen bei einer Eigendehnung den Vorteil einer direkten kinästhetischen Rückmeldung an. Das bedeutet, dass man über „... die kinästhetischen Rückmeldungen aus der gedehnten und der zur Dehnung eingesetzten Muskulatur die direkte und uneingeschränkte Möglichkeit der sensorisch-motorischen Handlungsregulation" hat (ebd., S. 10). Auf diesen Vorteil verweisen Glück, Schwarz, Hoffmann und Wydra (2002) in einer Untersuchung, in der sie zeigen konnten, dass durch Eigendehnung eine höhere Beweglichkeit erreicht werden konnte als durch eine Fremddehnung. 


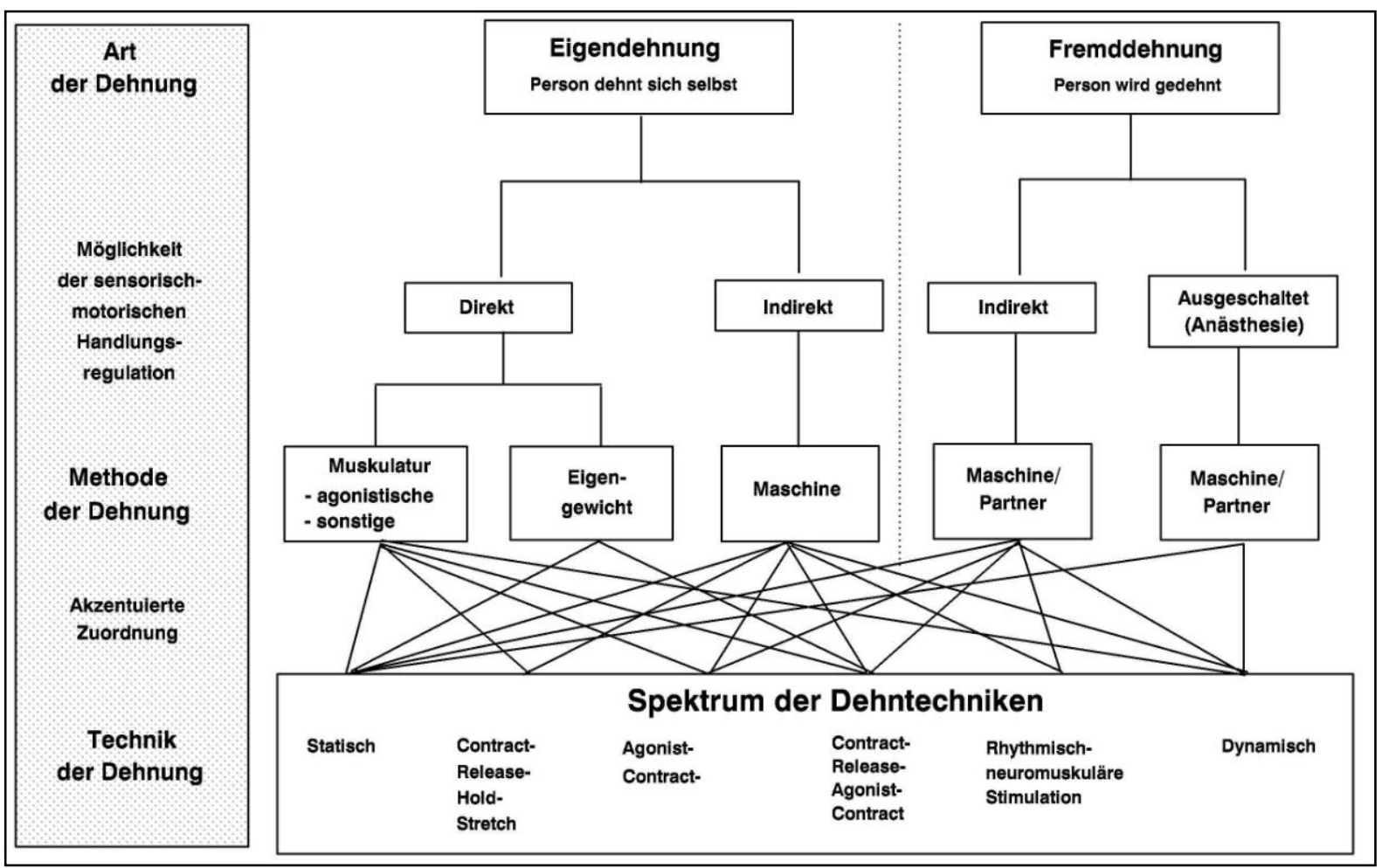

Abb. 1 Differenzierung der verschiedenen Formen der Muskeldehnung (Wydra et al., 1999, S. 11)

Eine Muskeldehnung beziehungsweise ein Dehntraining lässt sich nach Wiemann (1994, S. 55) zeitlich eingrenzen: „Die erste Gruppe der singulären Dehnungsmaßnahmen behandelt einzelne bzw. nur in wenigen Wiederholungen durchgeführte Dehnungen. Kurzzeitiges Dehnen stellt demgegenüber ein 15bis 30-minütiges Dehnprogramm dar, das aus mehreren Sätzen mit mehrfachen Wiederholungen von Dehnungsprozeduren für einen Muskel oder eine Muskelgruppe zusammengesetzt ist, so wie es in der Therapie oder in der Trainingspraxis durchgeführt wird. Langzeitige Dehnungsprogramme enthalten über mehrere Tage oder Wochen täglich oder mehrmals wöchentlich zu absolvierende Kurzzeitprogramme". Ein Dehntraining wird in der hier vorliegenden Arbeit in Anlehnung an die vorausgegangene zeitliche Einteilung als ein langzeitiges Dehnungsprogramm verstanden.

Wie ein Dehntraining gestaltet werden sollte, darüber können keine allgemeingültigen Empfehlungen ausgesprochen werden. Anders als bei einem Krafttrai- 
ning, für das überwiegend einheitliche Handlungsempfehlungen hinsichtlich der Belastungsparameter existieren (vgl. Fröhlich, Links, \& Pieter, 2012), fehlen solche konkreten Empfehlungen für das Dehntraining. In der Vergangenheit erklärte Marschall (1999, S. 5), dass „... widersprüchliche Handlungsempfehlung in der uneinheitlichen und teilweise fehlenden Beschreibung der für die Beweglichkeit relevanten Belastungsparameter zu suchen" sind. Weiter heißt es, dass insbesondere für die Trainingsanpassungen die „... essentiellen $\mathrm{Pa}$ rameter Intensität, Dauer, Dichte und Häufigkeit einer Dehnbelastung nicht hinreichend präzisiert sind ..." (ebd.).

Schönthaler und Ohlendorf (2002, S. 22 - 27) zeigten im Rahmen einer Literaturrecherche, dass die Empfehlungen der Belastungsparameter für die Dehndauer in der Trainingspraxis zwischen zwei und 45 Sekunden, der Dehnungsumfang zwischen einer und sechs Serien, die Dehndichte zwischen zehn und 120 Sekunden, die Trainingshäufigkeit zwischen drei und sieben Tagen pro Woche variieren. Es existieren durchaus Untersuchungen, die verschiedene Belastungsparameter miteinander vergleichen und hieraus Handlungsempfehlungen ableiten. Beispielsweise soll nach Bandy, Irion und Briggler (1997) sowie Pollock et al. (1998) ein Muskel 10 bis 30 Sekunden und bei älteren Erwachsenen bis zu 60 Sekunden gedehnt werden. Ein Dehntraining sollte in Anlehnung an Decoster, Cleland, Altieri und Russell (2005) mindestens zwei- bis dreimal pro Woche durchgeführt werden. Das American College of Sports Medicine empfiehlt ein Dehntraining von mindestens drei bis vier Wochen (Garber et al., 2011). Die abhängige Variable in diesen Studien war das Erreichen einer maximalen Beweglichkeit. Jede der vorgenannten Studien zeigte eine Verbesserung der Beweglichkeit, allerdings mit unterschiedlicher Ausprägung. Selbst eine einmalige Dehnungsintervention führt zu einer signifikant höheren Beweglichkeit, sowohl bei jüngeren als auch bei älteren Personen (Ryan et al., 2014). Darüber hinaus scheint die gesamte Dehndauer wichtiger zu sein als der Dehnungsumfang. Johnson, Mitchell, Meek und Feland (2014) verglichen zwei Belastungsprotokolle (10 mal $9 \mathrm{sec}$. statisches Dehnen vs. einmal $90 \mathrm{sec}$. statisches Dehnen) bezüglich des Erreichens einer maximalen Beweglichkeit. Das Dehntraining wurde an sechs Tagen pro Woche über einen Zeit- 
raum von sechs Wochen an der ischiocruralen Muskulatur durchgeführt. Sie konnten zeigen, dass beide Belastungsprotokolle die Beweglichkeit signifikant steigerten und dass es zwischen den Belastungsprotokollen keinen Unterschied gab.

Bezüglich der Dehndichte verglichen Freitas et al. (2015) eine fünfmalige 90sekündige Dehnung der ischiocruralen Muskulatur mit und ohne Pause zwischen den einzelnen Wiederholungen ( 0 sec. Pause vs. $30 \mathrm{sec}$. Pause). Sie stellten fest, dass die Dehnung ohne Pause zwischen den fünf Wiederholungen eine signifikant höhere Steigerung der Beweglichkeit bewirkte als mit Pause. Freitas et al. (2015, S. 191) schließen daraus, dass eine Pause zwischen einzelnen Wiederholungen nicht nötig ist, um in kurzer Zeit die Beweglichkeit zu steigern.

Eine besondere Problematik stellt die Erfassung der Intensität dar. Bei einem Krafttraining kann die Intensität beispielsweise durch ein konzentrisches Muskelversagen konkret angegeben werden. Bei einem Dehntraining ist die Intensität stark vom subjektiven Schmerzempfinden abhängig (Marshall \& Siegler, 2014; Weppler \& Magnusson, 2010). Marschall (1999) verglich die Effekte von zwei unterschiedlichen Dehnintensitäten. Die Intensitätsstufen waren ein „weiches Dehnen“ und ein "maximales Dehnen“. Weiches Dehnen wurde als submaximale Intensität („deutlich spürbares Dehngefühl an der gedehnten Muskulatur“) und maximales Dehnen als ein Dehnen an der Schmerzgrenze („größtmögliches Dehngefühl“) definiert. Beide Intensitäten führten zu einer verbesserten Beweglichkeit. Allerdings war die maximale Intensität dem weichen Dehnen überlegen. Ebenso konnte in einer neueren Untersuchung nach einem vierwöchigen Dehntraining der ischiocruralen Muskulatur kein Unterschied zwischen zwei verschiedenen Dehnintensitäten hinsichtlich des Erreichens einer maximalen Beweglichkeit festgestellt werden (Muanjai et al., 2017). Die Dehnintensitäten waren bei Muanjaj et al. (2017) ähnlich wie bei Marschall (1999) in ein maximales Dehnen („point of pain“) und ein submaximales Dehnen („point of discomfort") unterteilt. Im Gegensatz zu Marschall (1999) scheint nach Muanjai et al. (2017) ein maximales Dehnen gegenüber einem submaximalen Dehnen keinen besonderen Vorteil zu haben. Allerdings ist ein direkter Ver- 
gleich dieser Studien problematisch, da eine Dehnintensität nur schwierig zu standardisieren ist. Eine Möglichkeit der Standardisierung wäre eine relative Dehnintensität ausgehend von der individuell maximal erreichten Beweglichkeit. Allerdings lässt sich dies im Rahmen einer langzeitigen Dehnstudie nur schwer realisieren. Freitas, Vilarinho et al. (2015) verglichen verschiedene Dehnintensitäten bei einer Dehnung der ischiocruralen Muskulatur, die relativ zur maximal zu ertragenden Dehnungsspannung errechnet wurde (50\%, 75 \%, $100 \%)$. Im Ergebnis stellten sie fest, dass sich durch eine relative Dehnintensität von $50 \%$ und $75 \%$ die Beweglichkeit nicht verbesserte.

\subsection{Einflussfaktoren auf die Beweglichkeit}

Die Einflussfaktoren auf die Beweglichkeit lassen sich in interne und externe Faktoren unterteilen (Alter, 2004, S. 147; Klee \& Wiemann, 2005, S. 11). Zu den internen Einflussfaktoren zählen das Geschlecht, das Alter, die Psyche sowie anatomische und neurophysiologische Parameter. Zu den externen Einflussfaktoren gehören die Tageszeit und die Temperatur. Die anatomischen und die neurophysiologischen Einflussfaktoren sowie das Alter bedürfen im Rahmen der hier vorliegenden Arbeit einer ausführlicheren Darstellung. Deshalb werden die anatomischen Einflussfaktoren in Kapitel 2.2.1, die neurophysiologischen Einflussfaktoren in Kapitel 2.2.2 sowie das Lebensalter in Kapitel 2.2.3 ausführlich dargestellt.

Das Geschlecht hat einen Einfluss auf die Beweglichkeit zugunsten der Frauen. Hier spielen in Anlehnung an Alter (2004, S. 120) unter anderem anatomische sowie hormonelle Unterschiede eine Rolle. Frauen zeigen einen höheren Östrogenspiegel sowie einen erhöhten Fettgewebsanteil und eine geringere Muskelmasse als Männer. Aus diesem Grund weisen Frauen eine geringere Gewebedichte auf, die zu einer verbesserten Beweglichkeit im Gegensatz zu Männern führt (Thienes, 2000, S. 56). Des Weiteren zeigen Männer gegenüber Frauen eine höhere Stiffness aufgrund einer höheren Muskelmasse (Blackburn, Bell, Norcross, Hudson, \& Kimsey, 2009; Blackburn, Riemann, Padua, \& Guskiewicz, 2004). Marshall und Siegler (2014) beschreiben darüber hinaus 
bei Frauen eine höhere Toleranz gegenüber dem Dehnungsschmerz während einer Muskeldehnung, sodass sie gegenüber Männern bei einer gleichen Dehnungsübung eine größere Beweglichkeit erreichen.

Die Psyche kann den Muskeltonus durch private, berufliche oder sportliche Stressfaktoren beeinflussen (Thienes, 2000, S. 59). Da nach Freiwald (2009, S. 237) der psychische Zustand mit nervösen und hormonellen Veränderungen gekoppelt ist, haben psychische Faktoren Einfluss auf die Muskelaktivität. Lundberg et al. (1994) untersuchten die Auswirkungen von mentalem Stress auf die EMG-Aktivität des M. trapezius. Sie fanden heraus, dass der erzeugte Stress unter anderem einen signifikanten Einfluss auf die Herzfrequenz, den Blutdruck sowie auf die EMG-Aktivität der Muskulatur hatte. Freiwald (2009, S. 236) konnte ebenfalls den Einfluss von subjektiver Entspannung auf die elektrische Aktivität einer gedehnten Person zeigen. Glück (2005) untersuchte im Rahmen ihrer Dissertation unter anderem den psychischen Einfluss von Stress auf die Beweglichkeit. Sie konnte zeigen, dass sich die Muskelaktivität unter Stresseinfluss und die maximale Beweglichkeit im Vergleich zu einer Kontrollgruppe nicht unterschieden. Allerdings beschreibt Glück (2005, S. 217), dass die Stresseinwirkung möglicherweise zu gering war, um ein hohe psychische Beanspruchung auszulösen.

Die Tageszeit hat aufgrund des zirkadianen Rhythmus Einfluss auf die Beweglichkeit (Freiwald, 2009, S. 140). Ensink, Saur, Frese, Seeger und Hildebrandt (1996) zeigten, dass die Wirbelsäulenbeweglichkeit über den Tag, von morgens bis abends, sukzessive zunimmt. Grosser (1977) konnte in einer Untersuchung zeigen, dass die Hüft-Rumpf-Beweglichkeit um neun Uhr morgens schlechter ausgeprägt war als um $18 \mathrm{Uhr}$ abends. Allerdings weist Thienes (2000, S. 57) darauf hin, dass aus der tageszeitlichen Abhängigkeit der Beweglichkeit nicht fälschlicherweise auf eine gleichzeitig bessere Trainierbarkeit der Beweglichkeit zu unterschiedlichen Tageszeiten geschlossen werden darf. 
Die Temperatur hat einen negativen Einfluss auf die Beweglichkeit, wenn sich beispielsweise eine Person bei niedrigen Temperaturen im Freien aufhält (Thienes, 2000, S. 56). Ist ein Muskel erwärmt, entweder passiv durch höhere Außentemperaturen oder aktiv durch Bewegung, so hat dies einen positiven Einfluss auf die Beweglichkeit. Dieser positive Effekt lässt sich auf einen geringeren Dehnungswiderstand zurückführen (Freiwald, 2009, S. 257). Ein geringer Dehnungswiderstand aufgrund einer verminderten Stiffness durch eine aktiv aufgewärmte Muskulatur bestätigt ebenfalls Knudson (2006, S. 7).

Die Beweglichkeit ist neben den oben genannten Einflussfaktoren abhängig von der Dehnfähigkeit der Skelettmuskulatur, den Sehnen und Bandstrukturen sowie der Gelenkigkeit, die anatomisch-strukturell limitierend wirkt (Alter, 2004; Klee \& Wiemann, 2005). Wydra (2009) entwickelte ein Strukturmodell der Beweglichkeit, dessen einzelne Komponenten in den nachfolgenden Kapiteln beschrieben werden (Abb. 2)

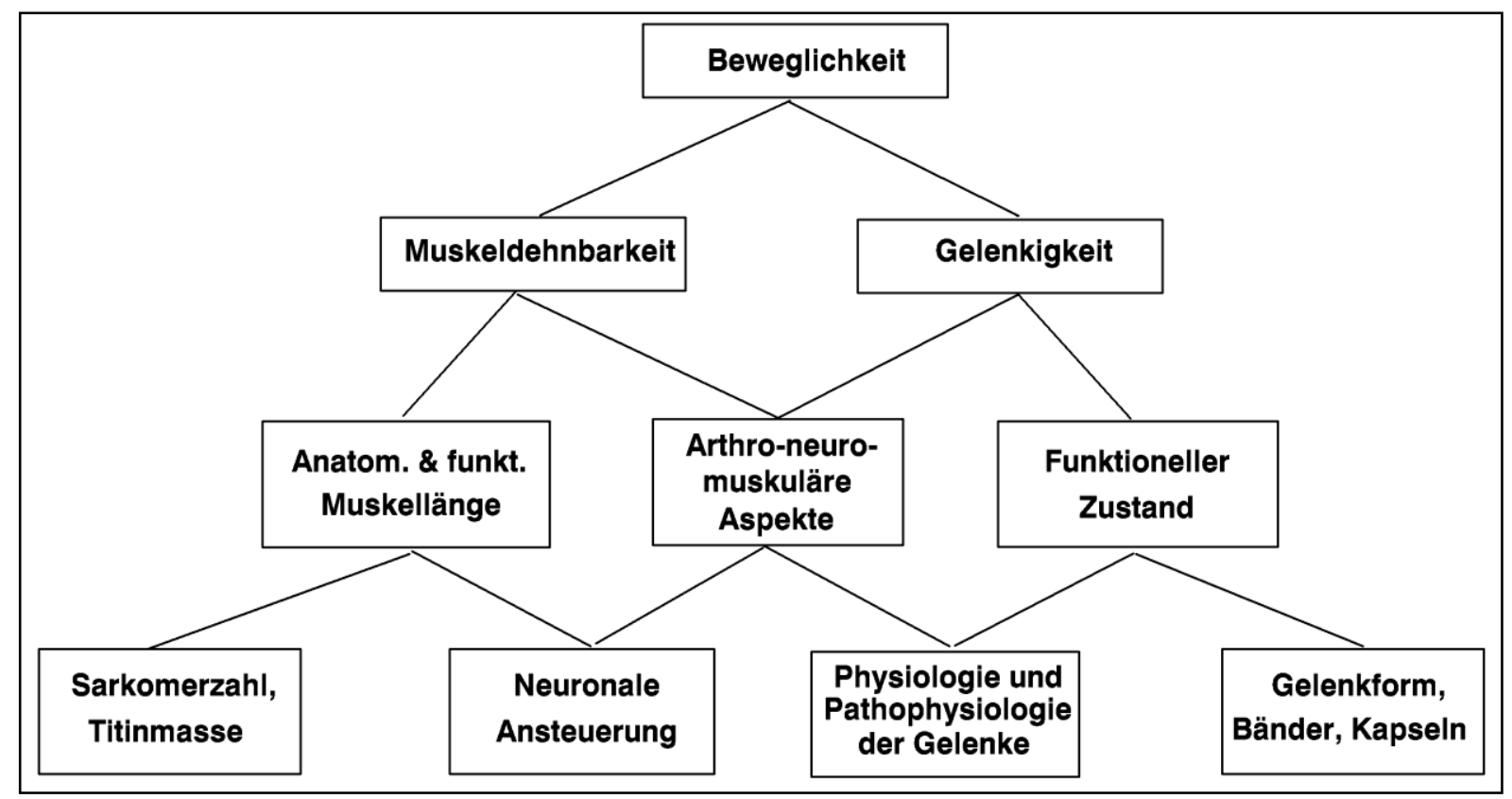

Abb. 2 Strukturmodell der Beweglichkeit (Wydra, 2009, S. 187) 


\subsubsection{Anatomische Einflussfaktoren}

Johns und Wright (1962, S. 824) zeigten anhand des Handgelenks einer Katze folgende anatomische Einflussfaktoren auf die Beweglichkeit: Gelenkkapsel $47 \%$, Muskulatur inklusive Faszien $41 \%$, Sehne $10 \%$, Haut $2 \%$. Die Autoren beschreiben die Ähnlichkeit mit den genannten limitierenden Einflussfaktoren auf die Beweglichkeit beim Menschen. Zudem geben sie an, dass diese erhobenen Daten nur für submaximale Bewegungsausmaße gelten. Würden die genannten Strukturen bis zu einer maximalen Beweglichkeit beansprucht werden, dann würde die Sehne einen durchaus prominenteren Einflussfaktor darstellen. Die genannten prozentualen Angaben sollen lediglich die Bedeutung anatomischer Strukturen als limitierende Einflussfaktoren verdeutlichen. Insbesondere unterschiedliche Gelenke sowie veränderte Strukturen im Lebensalter würden die anatomischen Einflussfaktoren anders gewichten (de Araújo, 2004, S. 6).

Die Gelenkigkeit wird bestimmt durch die Form des Gelenkes und dessen Freiheitsgrade (Martin, Carl, \& Lehnertz, 2001, S. 217). Je nach Gelenktyp können durch unterschiedliche Freiheitsgrade mehr oder weniger große Bewegungsamplituden erreicht werden. Zu unterscheiden sind in Anlehnung an Schiebler und Korf (2007, S. 163) Gelenktypen, die nachfolgend von der größten zur kleinsten Bewegungsamplitude genannt werden: dreiachsige Gelenke mit drei Freiheitsgraden (Kugelgelenk), zweiachsige Gelenke mit zwei Freiheitsgraden (Eigelenk, Sattelgelenk), einachsige Gelenke mit einem Freiheitsgrad (Scharniergelenk, Radgelenk). Die einzelnen Gelenkformen sind in Abbildung 3 übersichtlich dargestellt. Durch eine Muskeldehnung kann die Beweglichkeit durch Anpassungserscheinungen an der Skelettmuskulatur und dem Bindegewebe verbessert werden. Nicht beeinflussbar hingegen sind die knöchernen Strukturen eines Gelenkes. 


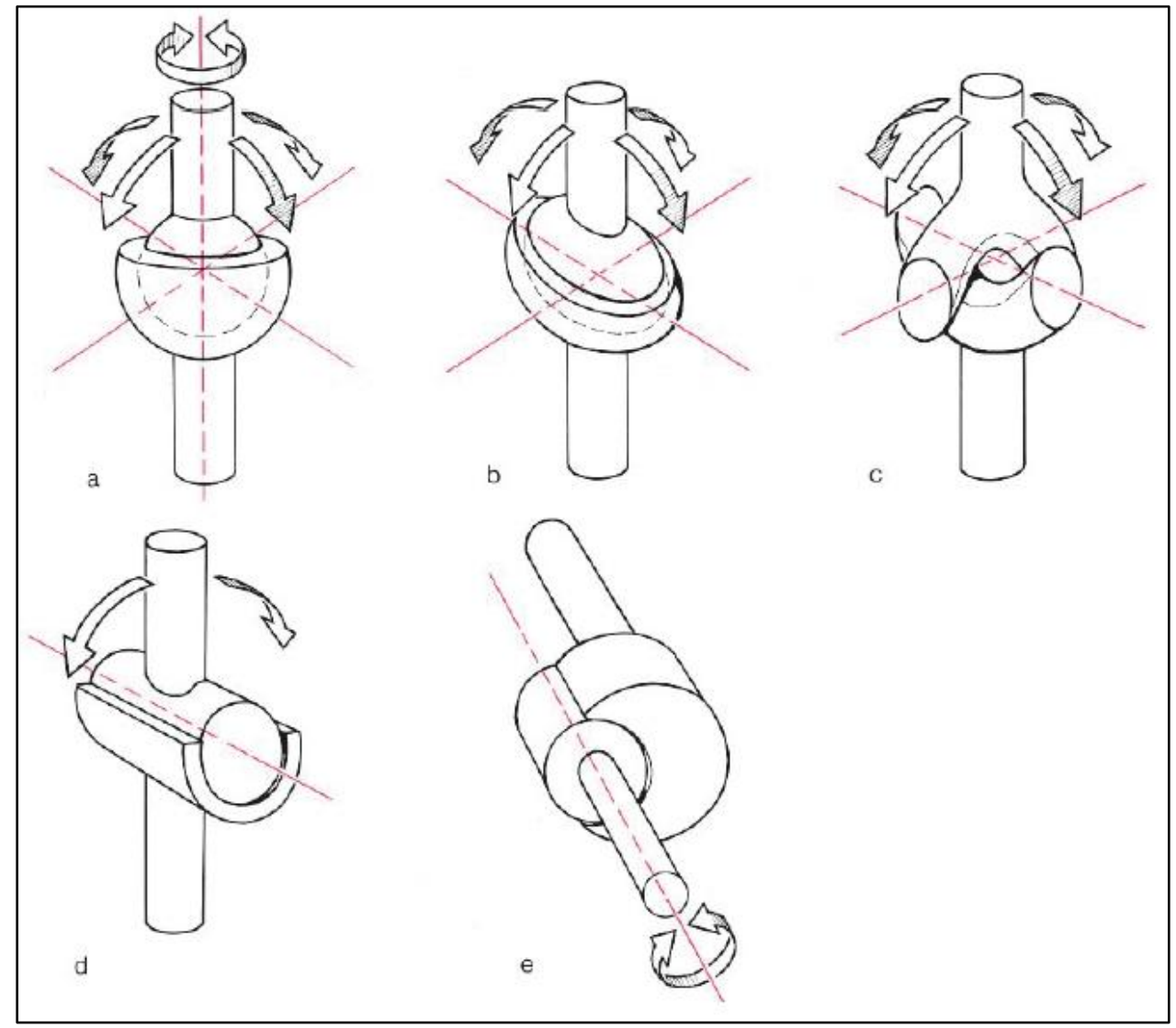

Abb. 3 Darstellung verschiedener Gelenktypen ( $a=$ dreiachsiges Gelenk, bspw. Schultergelenk als Kugelgelenk; $b=z$ weiachsiges Gelenk, bspw. proximales Handgelenk als Eigelenk; $c=$ zweiachsiges Gelenk, bspw. Grundgelenk des Daumens als Sattelgelenk; $d$ = einachsiges Gelenk, bspw. Mittel- und Endgelenk der Finger als Scharniergelenk; $e=$ einachsiges Gelenk, bspw. Gelenk zwischen Atlas und Axis als Radgelenk) (Schiebler \& Korf, 2007, S. 165)

Die Skelettmuskulatur besteht aus einer Vielzahl von quergestreiften Muskelfasern und längsangeordneten Myofibrillen (Abb. 4). Ultrastrukturell bestehen die Myofibrillen aus den Myofilamenten (Aktin- und Myosinfilament). Während einer Muskeldehnung beeinflussen die Aktin- und Myosinfilamente das Erreichen einer maximalen Beweglichkeit. Entsprechend der Filamentgleittheorie (Huxley, 2004; Huxley \& Hanson, 1954) schieben sich die Myofilamente bei einer Kontraktion ineinander (Muskelursprung und -ansatz nähern sich an) und bei einer Muskeldehnung gleiten sie auseinander (Muskelursprung und -ansatz entfernen sich), ohne dass sich die Länge der Myofilamente ändert. Es kommt zu einer mehr und weniger großen Überlappung der Aktin- und Myosinfilamente (Abb. 4, f und g). 


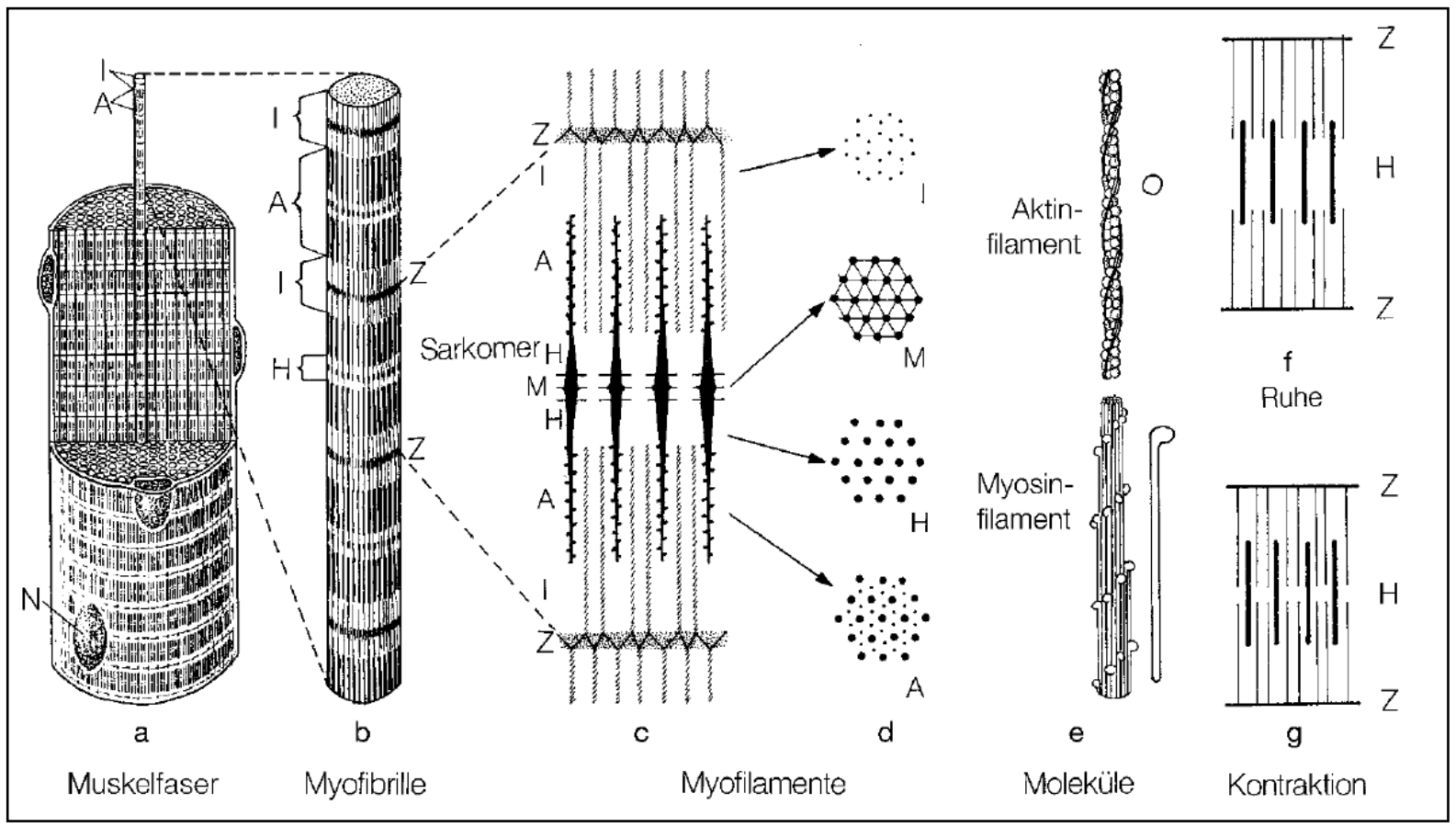

Abb. 4 Ultrastrukturelle Darstellung einer Muskelfaser (a) Quergestreifte Skelettmuskelfaser, $N=$ Nukleus, $I=$ helle Streifen einer Myofibrille, $A=$ dunkle Streifen einer Myofibrille; (b) Myofibrille mit I-, A-, $\mathrm{H}$ - und Z-Streifen; (c) Sarkomere von Z- zu ZStreifen mit dünnen Aktin- und dicken Myosinfilamenten; (d) Querschnitte durch die verschiedenen Segmente (I, M, H, A), (e) Molekularer Bau von Aktin- und Myosinfilamenten; ( $f$ ) Sarkomere in Ruhestellung, $(g)$ bei Kontraktion (Schiebler \& Korf, 2007, S. 62)

Wird ein Muskel gedehnt, so muss genau genommen von einer Dehnung der Muskulatur inklusive des Bindegewebes gesprochen werden. Zusammengefasst findet man den Begriff der Muskel-Sehnen-Einheit oder im englischsprachigen Raum die Muscle-Tendon-Unit (MTU). Holt, Pelham und Holt (2008, S. 114) beschreiben diesbezüglich ein Drei-Komponenten-Modell: den MuskelFaszien-Sehnen-Komplex (Muscle/Fascia/Tendon Complex). Demnach sind bei einer Muskeldehnung eine kontraktile und zwei elastische Komponenten beteiligt (Abb. 5). 


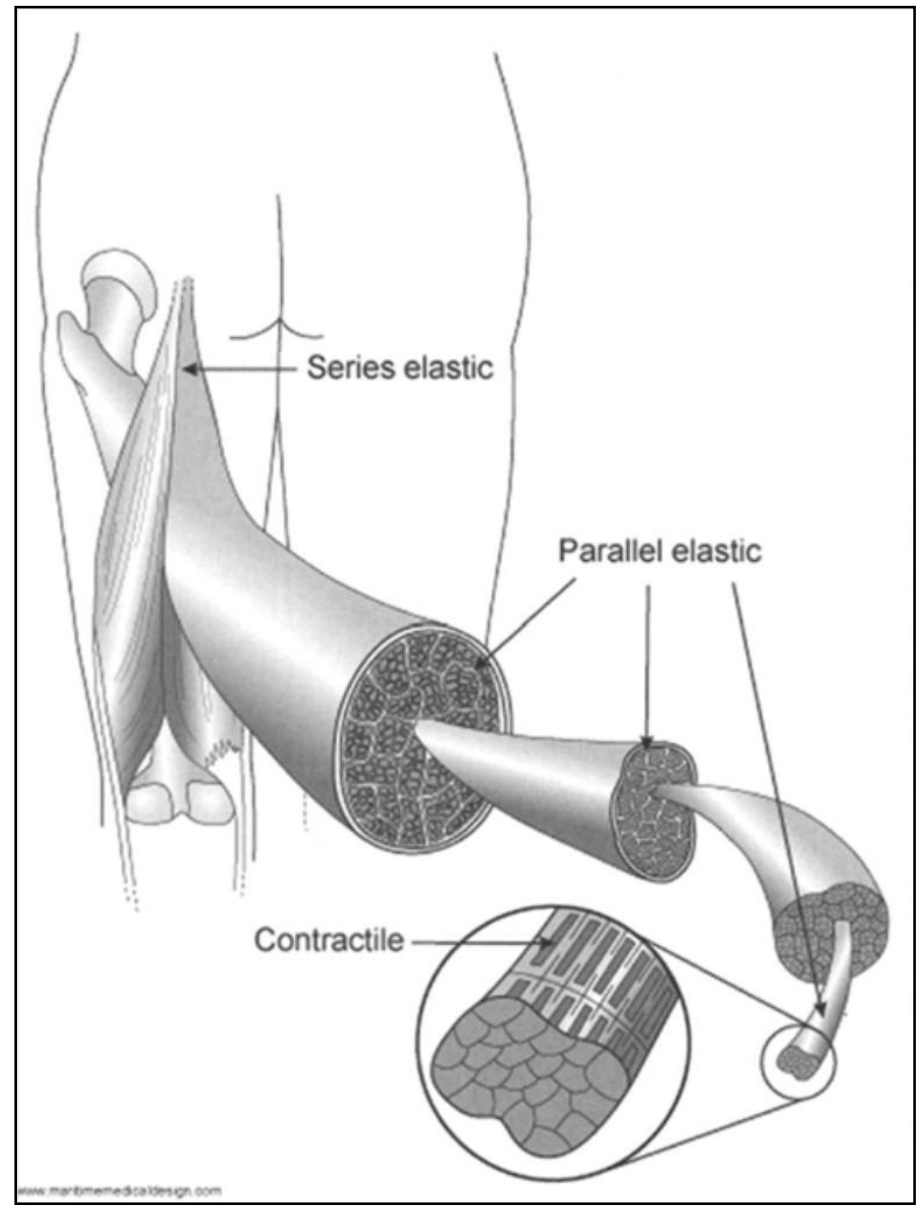

Abb. 5 Das Drei-Komponenten-Modell mit den seriell-elastischen, den parallelelastischen und den kontraktilen Komponenten (Holt et al., 2008, S. 114)

Die kontraktilen Komponenten dieses Drei-Komponenten-Modells stellen die in der Skelettmuskulatur befindlichen Sarkomere mit den Myofilamenten dar. Die zwei elastischen Komponenten sind die seriell-elastischen und parallelelastischen Elemente des Muskels. Mit den seriell-elastischen Elementen, die in Serie mit den kontraktilen Elementen liegen, sind primär die Sehnen gemeint (Alter, 2004, S. 63; Schomacher, 2005, S. 108). Sehnen bestehen wie auch Bänder aus parallelfaserigem dichten Bindegewebe (Schiebler \& Korf, 2007, S. 43). Die Sehnen bestehen in Anlehnung an Kjaer (2004, S. 652) aus Kollagen (65-80\%) und zu einem kleineren Teil aus Elastin (ca. $2 \%$ ). Kollagen besteht nach Alter (2004, S. 39) aus mehreren Grundsubstanzen, die als "cement substances" beschrieben werden: "They are composed of glycosaminoglycans (GAGs), plasma proteins, a variety of small proteins, and 
water" (ebd.). Da die GAGs eine hohe Wasserbindungskapazität aufweisen, besteht das Bindegewebe insgesamt zu etwa 60 -70\% aus Wasser (ebd.). Das in der Sehne vorkommende Kollagen besteht zu einem großen Teil aus Kollagen-Typ-I (ca. $60 \%$ ), das große Zugkräfte aushält. Die Kollagenfasern besitzen eine wellenförmige Struktur, die bei einem Muskelzug ausgeglichen werden und die Funktion eines mechanischen Puffers gegenüber einer auftretenden Dehnungsspannung wahrnehmen (Franchi et al., 2007). Das Elastin in der Sehne hat die Aufgabe äußere Kräfte abzufedern sowie die wellenförmige Anordnung der kollagenen Fasern nach einer Muskeldehnung wieder herzustellen (Butler, Grood, Noyes, \& Zernicke, 1978; Kannus, 2000). Die Sehnen bilden zusammen mit der Skelettmuskulatur eine funktionelle Einheit und übertragen Kräfte der Muskulatur über den Muskelsehnenansatz auf das Knochengewebe (Curwin, 2005; Knudsen et al., 2015). Der Muskelsehnenübergang stellt einen Bereich dar, in dem sich Muskelfasern mit kollagenen Fasern der Sehnen verbinden (Ciena et al., 2010). Die Verbindung zwischen Sehne und Muskulatur ist essentiell für eine gut funktionierende Muskel-Sehnen-Einheit, um Gelenkbewegungen zu ermöglichen. Der Muskelsehnenübergang reagiert sehr sensibel auf von außen wirkende Kräfte. Bleiben beispielsweise äußere Kräfte aus, so wird der Muskel-Sehnen-Übergang geschwächt und ist somit nicht mehr stark belastbar (Curwin, 2005, S. 79).

Die parallel-elastischen Elemente liegen parallel zu den kontraktilen Elementen und sind für die Ruhespannung der Muskulatur verantwortlich. Alter (2004, S. 63) sowie Horowits und Podolsky (1987) beschreiben hierbei das Titin als wichtigste Komponente (vgl. auch Linke, 2000; Maruyama, Kimura, Ohashi, \& Kuwano, 1981; Maruyama, Matsubara, Natori, Nonomura, \& Kimura, 1977; Tskhovrebova \& Trinick, 2010; Tskhovrebova, Trinick, Sleep, \& Simmons, 1997). Abbildung 6 zeigt, dass das Titinfilament mit dem Myosin- und Aktinfilament verbunden ist (Horowits \& Podolsky, 1987; Liversage, Holmes, Knight, Tskhovrebova, \& Trinick, 2001; Young, Ferguson, Bañuelos, \& Gautel, 1998). Außerdem reguliert das Titinfilament nach Whiting, Wardale und Trinick (1989) insgesamt die Filamentlänge und wirkt darüber hinaus als viskoelastischer Dämpfer und verhindert eine Überdehnung des Sarkomers (Bianco, 
Mártonfalvi, Naftz, Kőszegi, \& Kellermayer, 2015). Aufgrund der elastischen Eigenschaften der Titinfilamente sind sie in der Lage, nach einer Dehnung wieder in ihre Ruhespannungsposition zurückzukehren und verhalten sich somit wie eine mechanische Feder (Kellermayer, Smith, Granzier, \& Bustamante, 1997; Klee, Jöllenbeck, \& Wiemann, 2002; Politou, Thomas, \& Pastore, 1995; Wang, McCarter, Wright, Beverly, \& Ramirez-Mitchell, 1991).

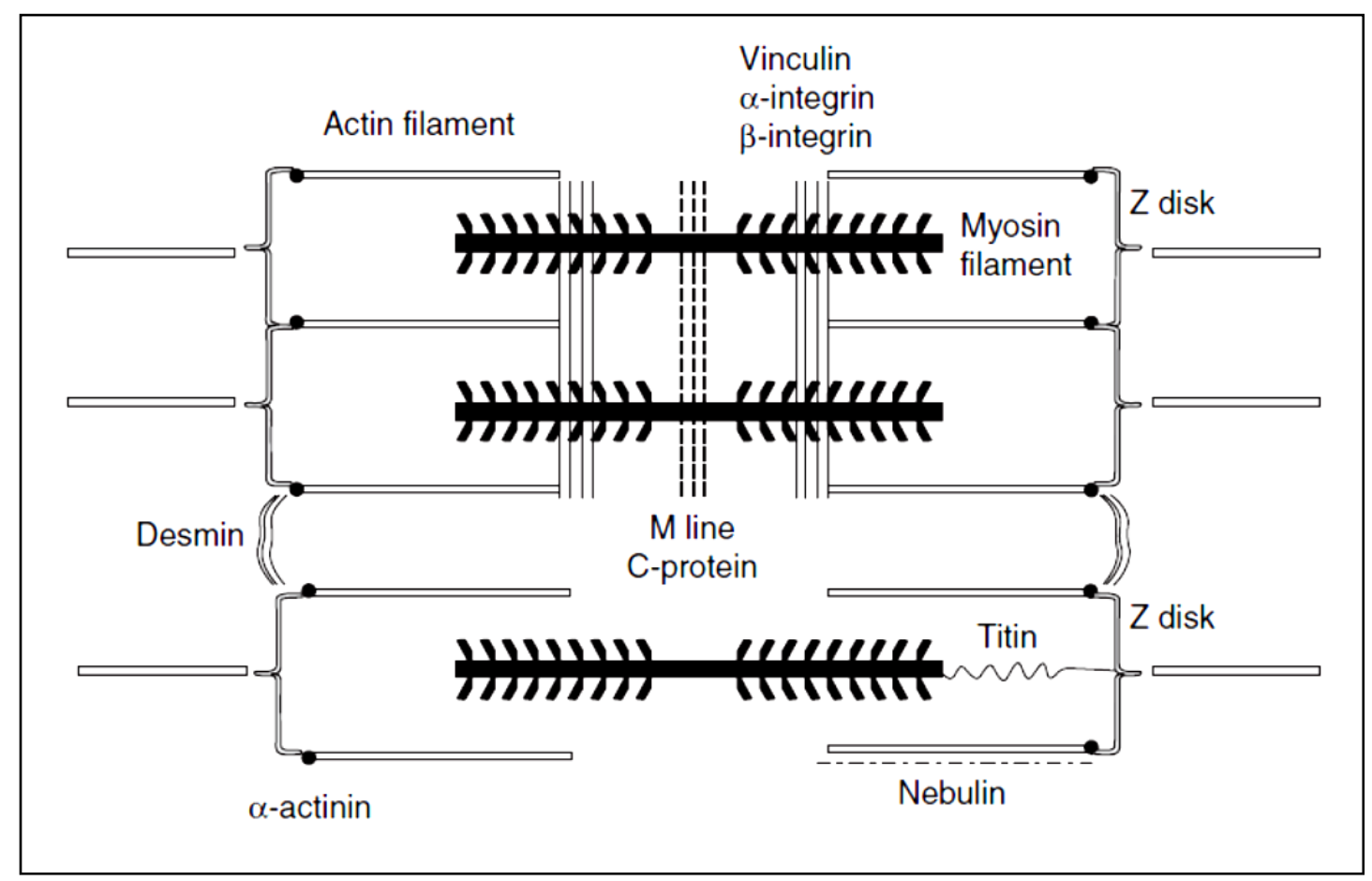

Abb. 6 Schematische Darstellung eines Sarkomers mit Myosin-, Aktin- und Titinfilament (Tricoli, 2011, S. 6)

Holt et al. (2008, S. 114) zählen die bindegewebigen Hüllen der Muskulatur (Faszien) zu den parallel-elastischen Komponenten. Faszien bestehen aus straffem Bindegewebe und stellen nach Schiebler und Korf (2007, S. 169) ein bindegewebiges Hüllsystem der Muskeln und Organe dar (vgl. auch Schleip, 2003). Sie sind darüber hinaus für die Übertragung von Kontraktionskräften zwischen einzelnen Muskelfasern zuständig (Purslow \& Delage, 2014). Des Weiteren können Faszien mehrere Muskeln überspannen, die dann als „myofascial chains“ beschrieben werden (Krause, Wilke, Vogt, \& Banzer, 2016; Myers, Frenk, \& Hamilton, 1997). Das Bindegewebe um und an der Skelett- 
muskulatur kann nach Purslow und Delage (2014, S. 4) als Muskelfaszien zusammengefasst werden. Die Anordnung der einzelnen Faszienstrukturen besteht in Anlehnung an Eckstein, Paulsen und Tilmann (2010, S. 90) in einer Art Schachtelsystem „... das von der Faszie auf der Muskeloberfläche über das Perimysium der Muskelfaserbündel bis zum Endomysium der einzelnen Muskelfaser reicht" (Abb. 7).

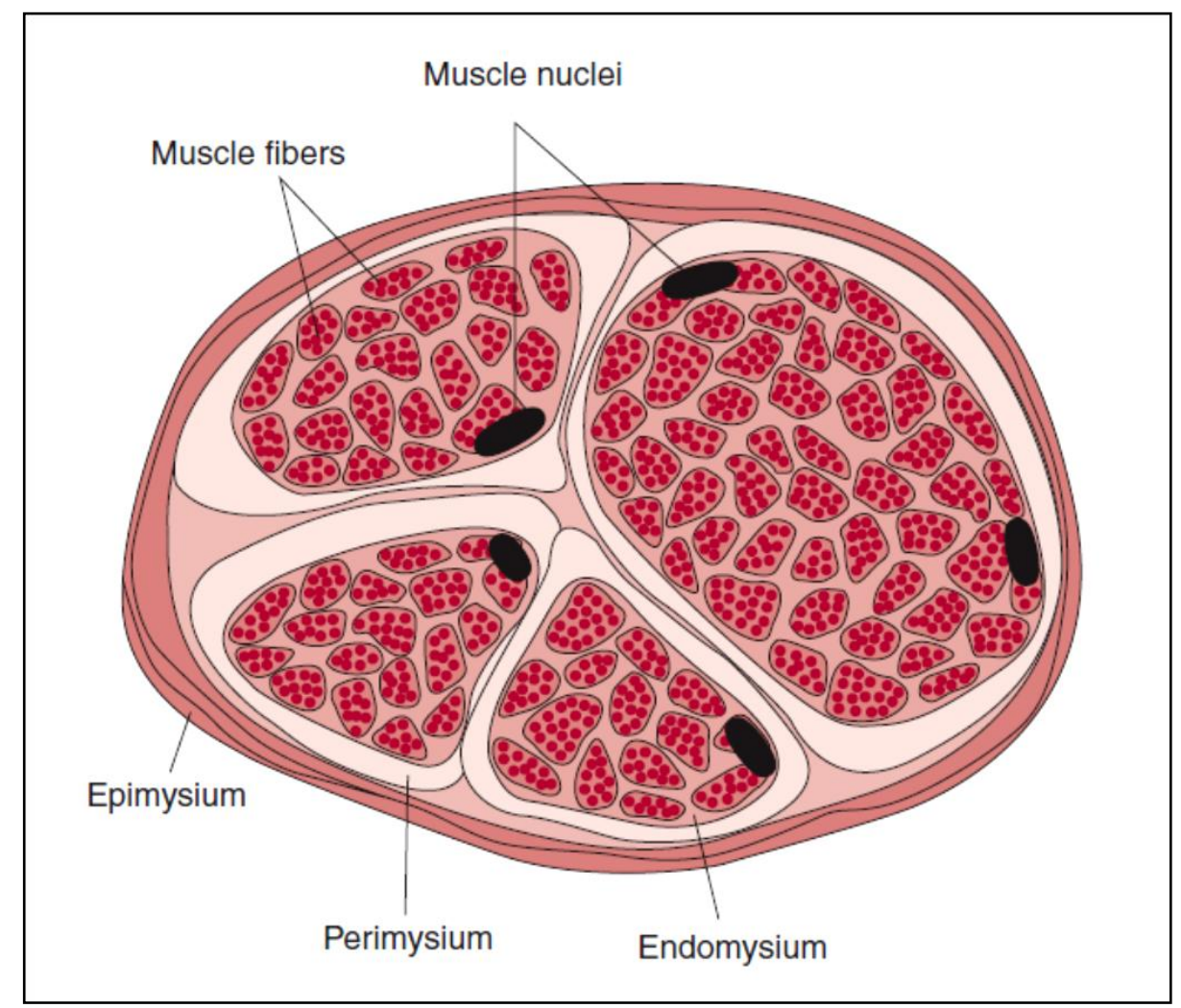

Abb. 7 Schematische Darstellung der Muskelfaszien (Chleboun, 2005, S. 121)

Die Muskulatur besteht in Anlehnung an Alter (2004, S. 49) zu ca. $30 \%$ aus Bindegewebe und beeinflusst die Muskulatur inklusive der Faszien zu ca. $41 \%$ in der Beweglichkeit (Johns \& Wright, 1962, S. 824). Das bedeutet, dass die Faszien einen wesentlichen Einflussfaktor auf die Beweglichkeit haben (Alter, 2004, S. 49). Insbesondere das Perimysium hat während einer Muskeldehnung nach Purslow (1989, S. 21) einen besonderen Einfluss auf die Dehnungsspannung, da die Muskelfaszie stärker durch perimysiales Bindegewebe als durch endomysiales Bindegewebe vertreten ist. Nach Schomacher (2005, S. 108) 
kommen die parallel-elastischen Einheiten „... frühestens unter Spannung, wenn der Muskel endgradig verlängert wurde. Dann werden sie zu den in Serie geschalteten elastischen Elementen hinzugezählt“.

\subsubsection{Neurophysiologische Einflussfaktoren}

Inwieweit ein Muskel dehnfähig ist, hängt mitunter von seiner Entspannungsfähigkeit ab. Ein möglichst wenig oder nicht aktiver Muskel ist wichtig, um eine Bewegung über eine maximale Bewegungsamplitude auszuführen (Regnier \& Cheng, 2016). Bei Körperbewegungen und auch Dehnungsvorgängen sind immer spezifische Rezeptoren beteiligt, die bei entsprechender Reizung aktiv werden und ,... die im zentralen Nervensystem subjektive Wahrnehmungen von Bewegung und das Gefühl von mechanischen Dehnungsspannungen im Muskel-Sehnen-Gelenksystem erzeugen" (Freiwald, 2009, S. 97). Es existieren zwei Typen sensorischer Rezeptoren, die das Zentralnervensystem mit Informationen über eine Längenänderung im Rahmen einer Dehnung der MuskelSehnen-Einheit informieren (Ghez \& Gordon, 1996, S. 518). Es handelt sich hierbei um die Muskelspindeln und die Golgi-Sehnenorgane (Abb. 8). Beide Rezeptoren sind für die sensomotorische Regulation der Muskeltätigkeit durch auslösende Muskel- und Sehnenreflexe wichtig, die durch eine Dehnung der Muskulatur bzw. der Sehne ausgelöst werden (Schnabel, 2005, S. 137).

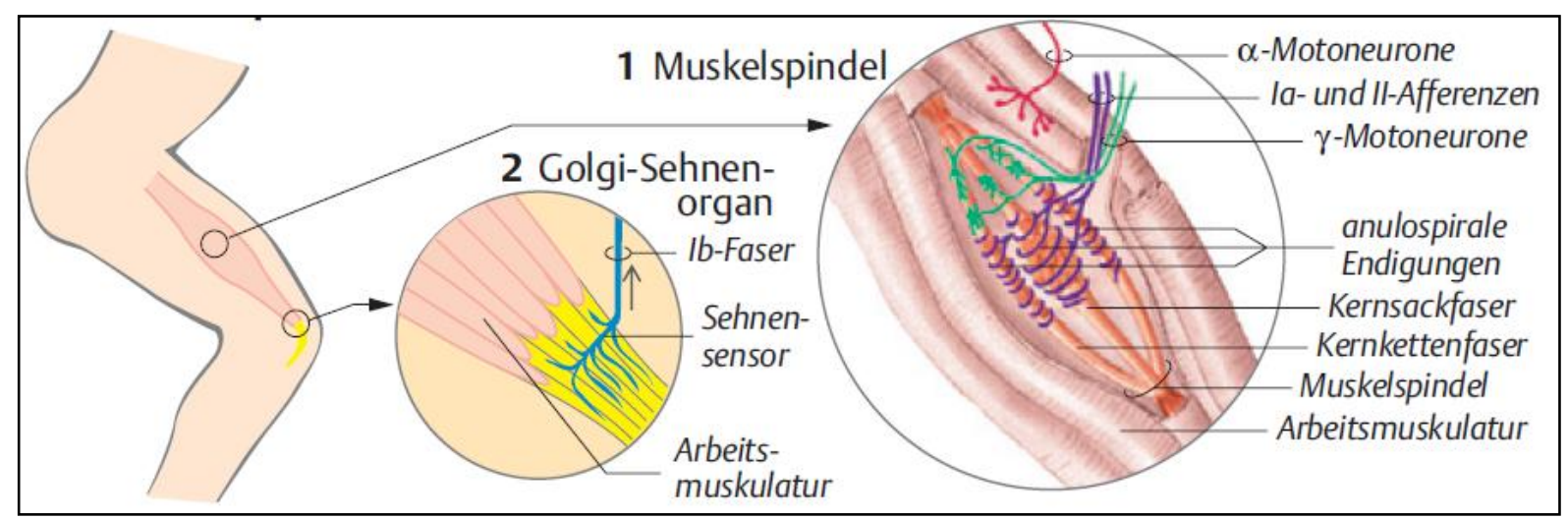

Abb. 8 Muskelspindel und Golgi-Sehnenorgan (Silbernagl \& Despopoulos, 2007, S. 319) 


\subsubsection{Muskelspindeln}

Die Muskelspindeln stellen die primären Dehnungsrezeptoren der Skelettmuskulatur dar (Matthews, 1964, 1972). Sie liegen parallel zu den extrafusalen Muskelfasern und sind kurze Muskelfasern, die von einer bindegewebigen Kapsel umgeben sind. In Anlehnung an Alter (2004, S. 75) werden die in der Kapsel liegenden Muskelfasern als intrafusale Muskelfasern und die eigentliche Arbeitsmuskulatur als extrafusale Muskelfasern bezeichnet (Abb. 9). Intrafusale Muskelfasern sind die Kernkettenfasern und die Kernsackfasern, die von afferenten Typ-la-Neuronen spiralförmig umwickelt sind (Abb. 8, rechts). Die Kernkettenfasern sind zusätzlich von afferenten Typ-II-Neuronen umwickelt (Silbernagl \& Despopoulos, 2007, S. 318). „Diese anulospiralen Endigungen sind die Sensoren für die (Längs-) Dehnung der intrafusalen Fasern und melden sowohl ihre Länge (la- und II-Afferenzen) als auch die Längenänderung (la-Afferenzen) zum Rückenmark“ (ebd.).

\subsubsection{Golgi-Sehnenorgane}

Die Golgi-Sehnenorgane bestehen aus kollagenen Bindegewebsfasern und einer sich zwischen diesen Fasern aufzweigenden markhaltigen Nervenfaser (de Mareés, 2003, S. 71). Sie werden von einer bindegewebigen Kapsel umhüllt und befinden sich hintereinander beziehungsweise in Serie zur extrafusalen Muskulatur (Lehmann-Horn, 2010, S. 130). Eine Besonderheit der GolgiSehnenorgane ist, dass sie nur afferent innerviert werden (Jami, 1992). Die intrafusalen Fasern der Muskelspindel hingegen werden afferent und efferent innerviert (Birbaumer \& Schmidt, 2003, S. 254). Die Nervenendigungen der Golgi-Sehnenorgane „... werden durch Zug der extrafusalen Muskelfasern an den Sehnensträngen durch Querkräfte gequetscht und dadurch aktiviert" (Lehmann-Horn, 2010, S. 131). 


\subsubsection{Einfluss einer Muskeldehnung auf die sensorischen Rezeptoren}

Die Muskelspindel und die Golgi-Sehnenorgane weisen ein unterschiedliches Antwortverhalten auf eine Muskeldehnung auf. Bei beiden Rezeptoren kann es zu Aktionspotentialen durch die afferenten Fasern kommen wenn der Muskel gedehnt wird. Eine Dehnung wird größtenteils von den Muskelfasern abgefangen, sodass die Golgi-Sehnenorgane nur in geringem Maße mechanisch deformiert werden. Aus diesem Grund feuern die afferenten Fasern der GolgiSehnenorgane wesentlich schwächer als die der Muskelspindeln (Ghez \& Gordon, 1996, S. 521). In Anlehnung an Lehmann-Horn (2010, S. 148) registrieren die Muskelspindeln die Länge und die Längenänderung eines Muskels. Die Sehnenorgane hingegen registrieren die Muskelspannung. Aus diesem Grund kommt es bei unterschiedlicher Beanspruchung der Muskulatur zu verschiedenen Entladungsmustern (Abb. 9 unten). 


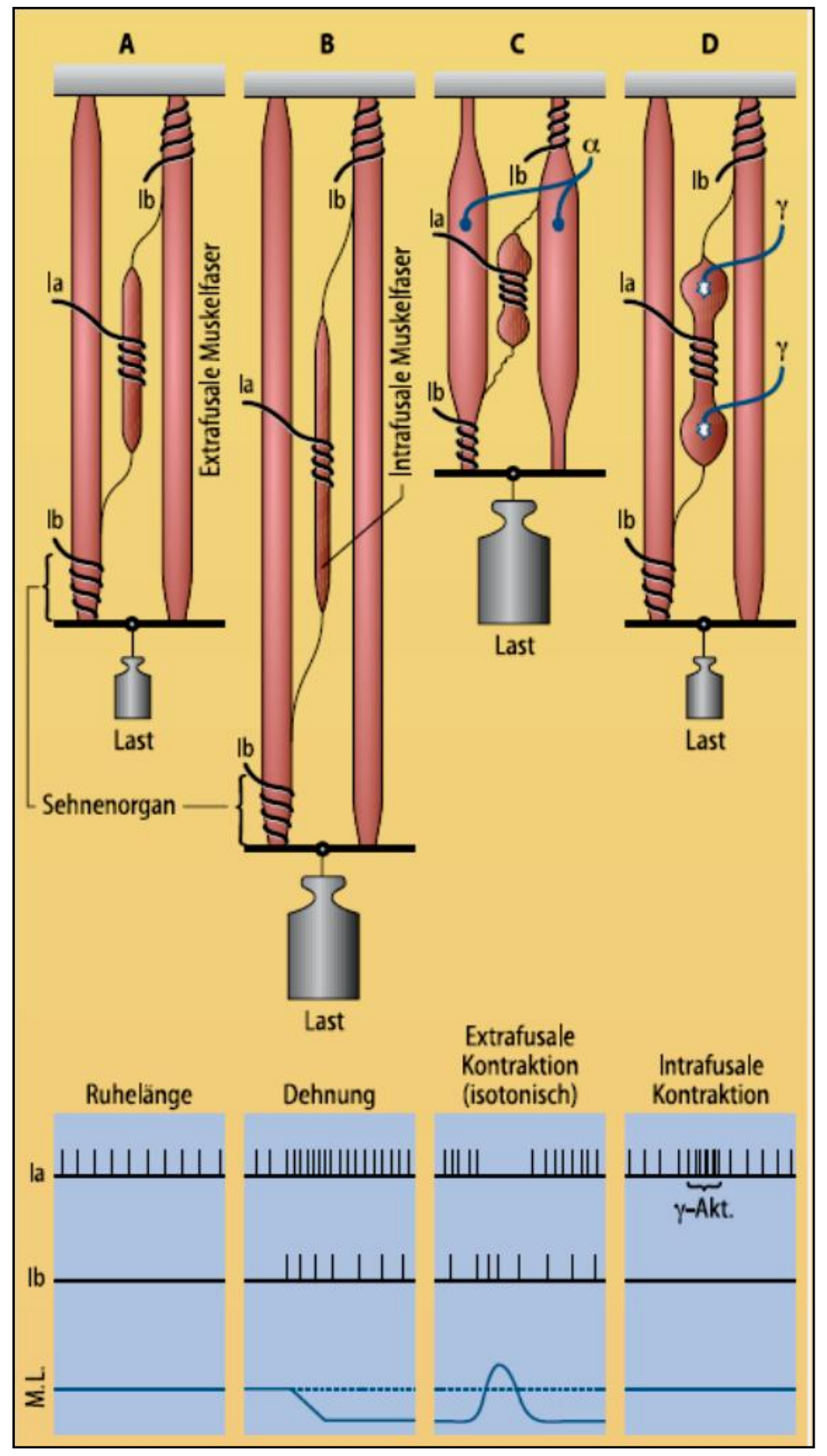

Abb. 9 Lage der Muskelspindeln und Golgi-Sehnenorgane (oben) und Entladungsmuster bei Beanspruchung der Muskulatur (unten) ( $A=$ Lage im Muskel in Ruhe; $B=$ Formveränderungen bei passiver Dehnung; $C=$ bei isotonischer Kontraktion der extrafusalen Muskelfasern; $D=$ bei alleiniger Kontraktion der intrafusalen Muskelfasern ( $\gamma$-Aktivierung); la = Entladungsmuster der primären Muskelspindelafferenzen über la-Fasern; Ib = Entladungsmuster der Sehnenorgane über Ib-Fasern; M.L. = Muskellänge) (Lehmann-Horn, 2010, S. 149)

Die Erfassung von Entladungsmustern beziehungsweise Aktionspotentialen in der Muskulatur erfolgt über die Elektromyographie mithilfe von Oberflächenelektroden auf dem Muskel, oder direkt im Muskel über Nadelelektroden. Bei 
einer Oberflächenelektromyographie (OEMG) werden Aktionspotentiale der Skelettmuskulatur gemessen, die ausgelöst durch einen DepolarisationsRepolarisationsablauf längs an der Muskelfasermembran entstehen. „Durch De- und Repolarisationsvorgänge an der Muskelfaseroberfläche entsteht ein elektrisches Signal, das der Elektromyographie zugrunde liegt. Die Zone, in der diese elektrischen Vorgänge ablaufen, beträgt ca. 1 bis $3 \mathrm{~mm}^{2}$ und bewegt sich mit einer Geschwindigkeit von 2 bis $6 \mathrm{~m} / \mathrm{s}$ entlang der Muskelfaser. Diese Zone wird als Dipol betrachtet" (Bochdansky, 1994, S. 62). Die OEMG-Signale werden bipolar durch die Spannungsdifferenz von zwei auf der Haut über dem Zielmuskel angebrachten Elektroden erfasst. Für die OEMG werden zwei Oberflächenelektroden nebeneinander in Faserlängsrichtung auf den Muskelbauch der Zielmuskulatur geklebt. Eine zusätzliche Oberflächenelektrode wird als Referenzelektrode verwendet, die auf ein nicht-aktives Gewebe geklebt wird. Hier bietet sich ein Knochenvorsprung in der Nähe des abzuleitenden elektromyographischen Signales an. Die zwei erfassten Signale der Muskulatur werden in Relation zu dem Signal der Referenzelektrode erfasst, anschließend verstärkt und ergeben somit das EMG-Signal (Abb. 10).

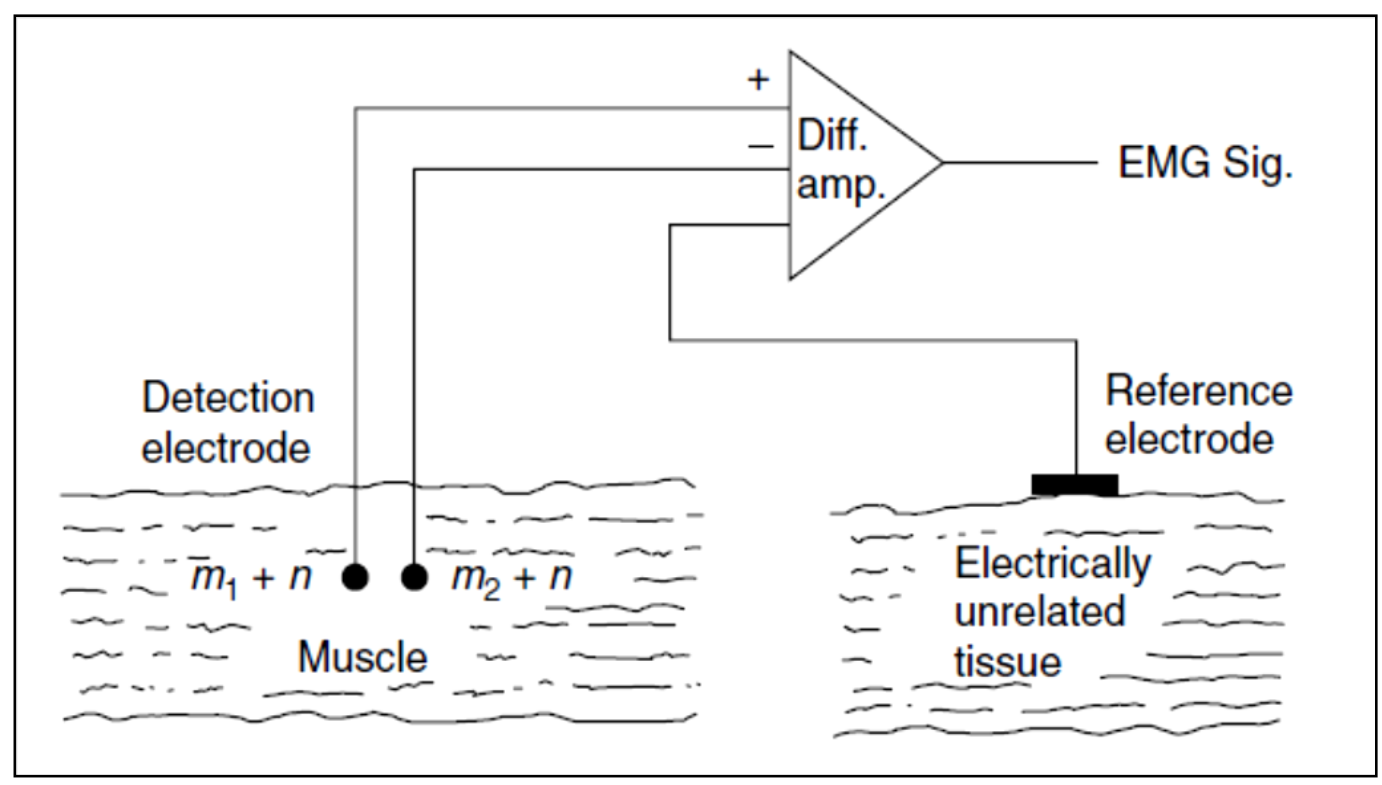

Abb. 10 Schematische Darstellung einer Oberflächenelektromyographie (zwei Oberflächenelektroden auf dem Zielmuskel - links unten; eine Oberflächenelektrode als Referenzelektrode - rechts unten) (De Luca, 2006, S. 105) 
In Anlehnung an Pfeifer, Vogt und Banzer (2003, S. 331) stellt ein gemessenes EMG-Signal „... ein extrazellulär abgeleitetes Summensignal aller Aktionspotentiale der aktiven motorischen Einheiten dar".

\subsubsection{Neuromuskuläre Dysbalance}

Aufgrund der nervösen Ansteuerung der Muskulatur kann das Nerv-MuskelZusammenspiel einen negativen Einfluss auf die Beweglichkeit haben. Nach Freiwald, Engelhardt und Reuter (1998, S. 167) steht das Nerv-MuskelZusammenspiel in einem nicht trennbaren Funktionszusammenhang zueinander. Dieser Funktionszusammenhang äußert sich in einer mehr oder minder ausgeprägten muskulären Balance. Während einer muskulären Balance nimmt das Gelenk eine Stellung ein, „... in der sich die Momente der Ruhespannung von Agonisten und Antagonisten die Waage halten" (Wiemann, Klee, \& Startmann, 1998, S. 115). Eine neuromuskuläre Dysbalance wird von Neumann (1998, S. 16) definiert als: „Abweichung vom normalen (individuellen) motorischen Stereotyp (Bewegungsprogramm), die durch zeitlich veränderte und instabile gezielt nervale Aktivierung einzelner Muskelgruppen zu Bewegungseinschränkung, Leistungsabnahme und/oder arthromuskulären Beschwerden führt". Freiwald, Engelhardt und Reuter (1998, S. 167) definieren die neuromuskuläre Balance als „... anforderungsgerechte Homöostase (Balance) aller an den arthronalen Systemen beteiligten nervösen und humoralen Funktionen und Strukturen ....". Eine muskuläre Dysbalance hingegen ist durch eine Störung dieser Homöostase gekennzeichnet (ebd.). In Anlehnung an Schomburg (1998, S. 40) können muskuläre Dysbalancen aufgrund pathologischer Prozesse, wie Störungen des Nervensystems oder Störungen des Bewegungsapparates, ausgelöst werden. Darüber hinaus können auch nicht-pathologische Ursachen, wie Fehlhaltungen im Alltags- und Berufsleben, ursächlich sein (ebd.). Als primäre Ursache einer muskulären Dysbalance geben Wiemann, Klee und Startmann (1998, S. 115) eine Hypertrophie oder eine Atrophie der gelenkwirksamen Muskeln an. Darüber hinaus beschreibt Wydra (2009, S. 189) eine Verringerung oder eine Erhöhung des Muskeltonus, die durch eine Störung im Gelenksystem ausgelöst werden kann, um ein Gelenk vor Überlastung zu schüt- 
zen. Ebenso beschreibt Nürnberger (1998, S. 35), dass aufgrund von entzündlichen oder traumatischen Veränderungen die Afferenzen der Gelenkrezeptoren negativ beeinflusst werden und durch eine vermehrte Ausschüttung von Nozizeptoren das entsprechende Gelenk ruhig gestellt wird, was wiederum zu einer Atrophie der gelenkumgebenden Muskeln führt.

\subsubsection{Lebensalter}

Altern bedeutet in einer offenen Definition nach Danner und Schröder (1994, S. 95) zeitabhängige, irreversible und vorhersagbare Veränderungen sowie in Anlehnung an Baltes und Baltes (1994, S. 10) eine altersbezogene Verringerung der biologischen Kapazität beziehungsweise Funktionstüchtigkeit. Spirduso, Francis und MacRae (2005, S. 5) unterscheiden in Anlehnung an Hershey (1984) und Busse (1969) einen Alterungsprozess (aging process) und einen Prozess des Alterns (process of aging). Diese in der nachfolgenden Definition beschriebenen Prozesse sind individuell ausgeprägt und beeinflussen sich gegenseitig, sodass sie nicht unabhängig voneinander gesehen werden können: „Aging processes (primary aging) are universal age-related changes within a species that are independent of disease or environmental influence. The process of aging (secondary aging) refers to clinical symptoms (the syndrome of aging) and includes the effects of environment and disease" (Spirduso et al., 2005, S. 5).

Nachfolgend wird zunächst die Veränderung der Beweglichkeit über das Lebensalter beschrieben und anschließend wird auf die anatomisch-strukturellen Veränderungen eingegangen.

Ältere Menschen zeigen zumeist schlechtere Beweglichkeitsmaße als jüngere, wie Wydra (2008) anhand des Finger-Boden-Abstandes zeigen konnte. Der Finger-Boden-Abstand ist ein globales Maß für die Beweglichkeit, mit dem die Dehnfähigkeit der Muskeln an Oberschenkelrückseite und Rücken sowie die Gelenkigkeit der Hüfte und der Wirbelsäule betrachtet werden kann. Wydra (2008) stellte aus Daten von Beck (2006) von insgesamt 252.591 Versuchspersonen die Ergebnisse des Finger-Boden-Abstandes dar. Vom Kindesalter (6 - 9 
Jahre) bis ins Jugend- und frühe Erwachsenenalter (15 - 18 Jahre) nahm die Beweglichkeit zu, um mit steigendem Lebensalter sukzessive wieder abzunehmen (Abb. 11). Am Finger-Boden-Abstand wird Kritik geübt, da dieser Test den limitierenden Einfluss des aktiven und passiven Bewegungsapparates nicht eindeutig differenzieren kann (Alter, 2004, S. 239). Ensink et al. (1996, S. 1341) beschreiben diesbezüglich „... it neither differentiates movement of the hip from that of the lumbar spine nor does it take into consideration different arm length". Unabhängig von den Ergebnissen von Wydra (2008) konnten auch andere Untersuchungen einen negativen Einfluss des Lebensalters auf die Beweglichkeit zeigen. Nonaka et al. (2002) untersuchten die Beweglichkeit des Hüft- und Kniegelenkes bei Personen im Alter zwischen 15 und 73 Jahren. Sie konnten zeigen, dass die Beweglichkeit der Hüfte mit zunehmendem Lebensalter abnimmt, wobei die Beweglichkeit des Kniegelenks annähernd unverändert bleibt. Dies lag an den anatomisch limitierenden Faktoren während der Testung dieser beiden Gelenke. Bei der Testung des Hüftgelenkes wirkte der Muskel-Band-Apparat eher limitierend als bei der Testung des Kniegelenkes. Gajdosik, Vander Linden und Williams (1999) zeigten bei einem Vergleich von drei Altersgruppen (30 Jahre, 50 Jahre, 73 Jahre), dass das Alter einen negativen Einfluss auf die maximale Beweglichkeit des Sprunggelenkes hat. Ryan et al. (2014) verglichen akute Effekte einer Dehnung an der Wadenmuskulatur zwischen zwei Altersgruppen (23 Jahre vs. 69 Jahre) und konnten zeigen, dass sowohl die maximale Beweglichkeit als auch die maximale Dehnungsspannung bei den älteren Probanden im Vortest geringer ausgeprägt war. Weitere Untersuchungen, die einen negativen Einfluss des Lebensalters auf die Beweglichkeit zeigen, existieren beispielsweise auch für die Halswirbelsäule (Kuhlman et al., 1993; Youdas et al., 1992) und für die Schulter (Allander, Björnsson, Ólafsson, Sigfússon, \& Thorsteinsson, 1974). 


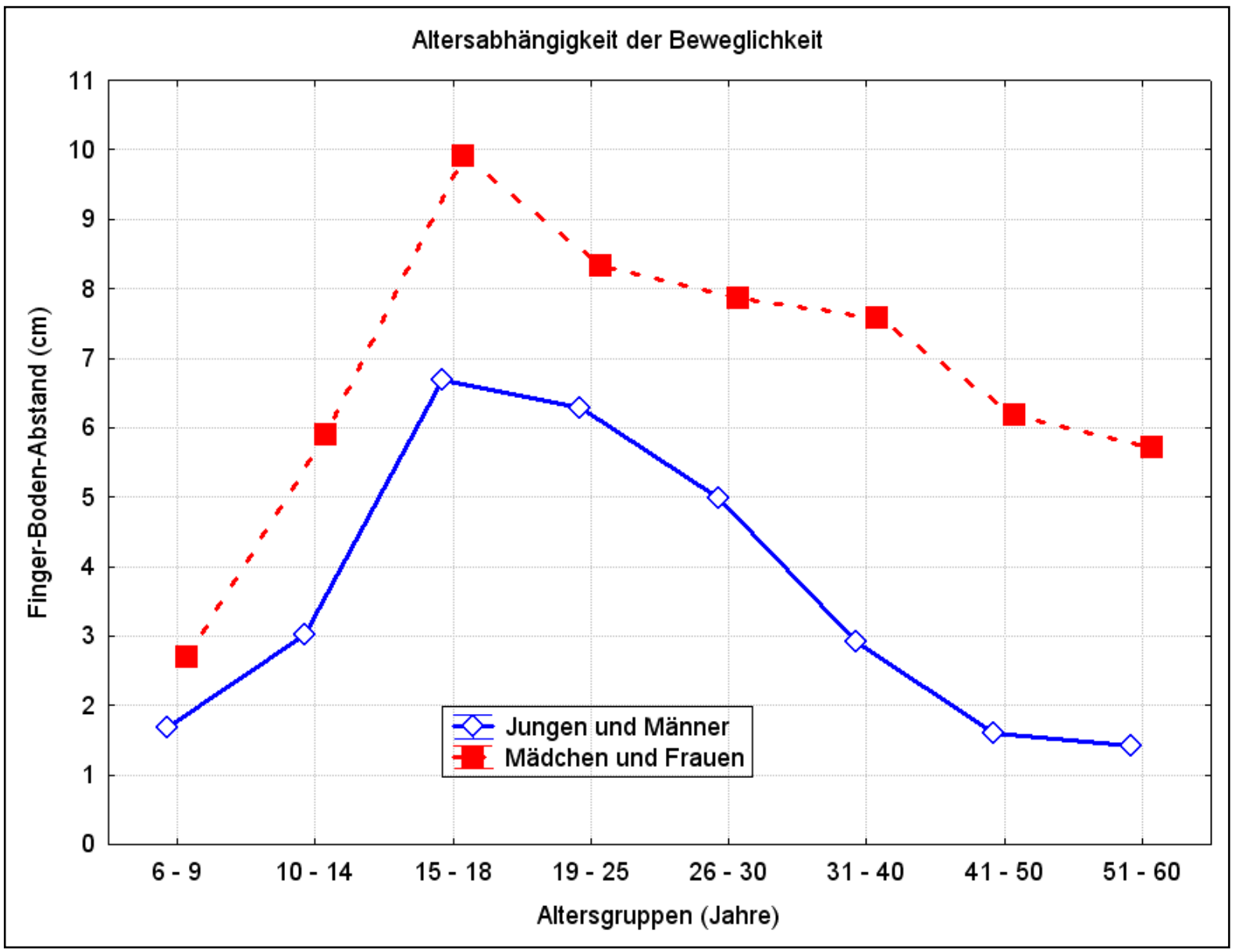

Abb. 11 Veränderung der Beweglichkeit im Alternsgang am Beispiel des Finger-BodenAbstandes (in Anlehnung an Wydra, 2009, S. 191)

Eine Abnahme der Beweglichkeit im Alternsgang besteht sowohl für Frauen als auch für Männer, wobei Frauen über die Lebensspanne insgesamt eine bessere Beweglichkeit zeigen (Grimston, Nigg, Hanley, \& Engsberg, 1993; Holland, Tanaka, Shigematsu, \& Nakagaichi, 2002; de Oliveira Medeiros, Araújo, \& Araújo, 2013). Die Verringerung der Beweglichkeit lässt sich auf Veränderungen in den Bereichen der Muskulatur und des Gelenksystems zurückführen (Holland et al., 2002; Nonaka et al., 2002). Veränderungen des Gelenksystems gehen zumeist mit einer Arthrose einher. Die Arthrose stellt einen Verschleiß eines Gelenkes beziehungsweise eine degenerative Gelenkerkrankung dar, und wird nach Krämer und Grifka (2005, S. 119) wie folgt definiert: „Degeneratives Gelenkleiden durch ein Missverhältnis zwischen Belastung und Belastbarkeit des Gelenkknorpels“. Pathologische Gelenkveränderungen wie die 
Arthrose, können mit Adaptionen im Sinne von muskulären Dysbalancen einhergehen (Wydra, 2009, S. 189). Nach Nuki (1999, S. 142) entwickelt sich die Arthrose, „... wenn das Gleichgewicht zwischen Abbau und Aufbau der Gelenkstrukturen gestört ist. Ein strukturelles Versagen des Gelenkknorpels kann die Folge abnormer mechanischer Belastungen eines gesunden Gelenkknorpels wie auch physiologischer Belastungen eines pathologisch gestörten Gelenkknorpels sein." Die Inzidenz der Arthrose ist nach Pereira et al. (2011) schwierig zu erheben. Dies liegt daran, dass die Definition einer Arthrose in der Literatur uneinheitlich ist. Sun, Stürmer, Günther und Brenner (1997, S. 190) beschreiben beispielsweise, dass nicht alle Patienten mit radiologisch diagnostizierter Arthrose auch klinisch erfassbare Symptome aufweisen. Felson et al. (1987, S. 916) konnten im Rahmen der Framingham Osteoarthritis Study zeigen, dass nur $40 \%$ der radiologisch diagnostizierten Arthrose mit klinischen Symptomen verbunden waren.

Mit zunehmendem Lebensalter nimmt die Muskelmasse ab (Lexell, 1995). Hierbei verringert sich sowohl die Größe als auch die Anzahl der Muskelfasern (Hooper, 1981; Lexell, Taylor, \& Sjöström, 1988). Lexell et al. (1988) beschreiben eine Abnahme der Muskelmasse ab ungefähr dem 25. Lebensjahr. Während die Muskelmasse abnimmt kommt es zu einer vermehrten Einlagerung von kollagenem Bindegewebe in der Muskulatur (Overend, Cunningham, Paterson, \& Lefcoe, 1992). Alter (2004, S. 31) beschreibt eine sehr geringe Compliance von Kollagen ,... which implies that small increases in the quantity of collagen in a muscle considerably increases the stiffness of the tissue". Neben der vermehrten Einlagerung von Bindegewebe in der Muskulatur lässt nach Krämer und Grifka (2005, S. 28) die Dehnbarkeit und die Elastizität von Sehnen, Bändern und Faszien „... aufgrund einer Verminderung der Anzahl elastischer Fasern nach". Insbesondere eine Vermehrung des perimysialen Bindegewebes der Muskulatur hat einen negativen Einfluss auf die Stiffness (Gajdosik, 1997, S. 164). Es kommt zusätzlich zu einem Verlust von Mukopolysacchariden und Wasser (Thienes, 2000, S. 56). „Mukopolysaccharide bestimmen durch ihre Eigenschaft, Wasser zu binden, maßgeblich das mechanische Verhalten des Gewebes bei Dehnung" (ebd.). 
Die Verminderung der Dehnbarkeit und Elastizität von kollagenem Gewebe sowie der Wasserverlust führen zu einer altersbedingten Zunahme der Stiffness. Chesworth und Vandervoort (1989) untersuchten die Stiffness der Wadenmuskulatur in drei verschiedenen Altersklassen (29 Jahre, 51 Jahre, 69 Jahre) bei Ansteuerung eines vorgegebenen Gelenkwinkels. Die Autoren konnten zwar eine höhere Stiffness bei den älteren Probanden im Vergleich zu der jüngsten Probandengruppe feststellen, allerdings war der Unterschied zwischen den drei Altersklassen nicht signifikant. Sobolewski, Ryan, Thompson, McHugh und Conchola (2014) untersuchten die viskoelastischen Eigenschaften der Wadenmuskulatur ebenfalls bei Ansteuerung eines vorgegebenen Gelenkwinkels zwischen jüngeren und älteren Personen (24 Jahre vs. 67 Jahre). Im Ergebnis konnte festgestellt werden, dass ältere Personen eine signifikant höhere Stiffness aufwiesen. Die genannten Untersuchungen lassen erkennen, dass die Studienlage bezüglich der Stiffness im Alter nicht eindeutig ist. Ein möglicher Grund liegt in den Voraussetzungen der Probanden. Bei Sobolewski et al. (2014) wurden die Probanden hinsichtlich ihrer physischen beziehungsweise sportlichen Ausgangslage nicht weiter spezifiziert. Bei Chesworth und Vandervoort (1989) wurde unter anderem als Einschlusskriterium das Erreichen eines bestimmten Gelenkwinkels genannt. Aus diesem Grund könnten diese Probanden eine homogenere Gruppe gebildet haben. Die Bedeutsamkeit der Ausgangsbedingungen der Probanden ist deshalb nicht zu unterschätzen, da insbesondere bei inaktiven Personen die Stiffness höher ausfallen kann. Bei Gajdosik (2001, S. 96) heißt es: „A minimal level of physical activity may prevent the accumulation of connective tissue in the aged muscle of active people".

Da es nach Wydra (2009, S. 195) aufgrund der Komplexität der Beweglichkeit determinierenden Faktoren schwierig ist eine Verminderung der Beweglichkeit auf differenzierte Veränderungen des passiven und aktiven Bewegungsapparates zurückzuführen, muss auch motorisches Verhalten berücksichtigt werden. Möglicherweise lässt sich eine Abnahme der Beweglichkeit durch eine verminderte körperliche Aktivität erklären. Stathokostas, McDonald, Little und Paterson (2013) untersuchten ältere Erwachsene in einem Lebensalter zwischen 55 
und 86 Jahren hinsichtlich der Beweglichkeit des Schulter- und Hüftgelenkes und des Einflusses der körperlichen Aktivität auf die Beweglichkeit. Sie konnten eine signifikante Abnahme der Beweglichkeit mit zunehmendem Lebensalter feststellen, allerdings hatte hier die körperliche Aktivität keinen signifikanten Einfluss auf die Beweglichkeit. Smith und Walker (1983) untersuchten die Beweglichkeit von Ellenbogen- und Kniegelenk bei Personen zwischen dem 55. und dem 84. Lebensjahr. Es konnte kein Zusammenhang zwischen der Beweglichkeit und der körperlichen Aktivität festgestellt werden. Morini, Bassi, Cerulli, Marinozzi und Ripani (2004) untersuchten die Hüft- und Kniebeweglichkeit zwischen zwei Altersgruppen (19 Jahre vs. 64 Jahre), wobei die ältere Untersuchungsgruppe in zwei Subgruppen unterteilt wurde (nicht aktiver Lebensstil vs. aktiver Lebensstil). Des Weiteren wurde der Einfluss der körperlichen Aktivität auf die Beweglichkeit untersucht. Im Ergebnis konnte festgestellt werden, dass die jüngere Altersgruppe eine bessere Beweglichkeit aufwies. Außerdem zeigte die ältere Untersuchungsgruppe mit aktivem Lebensstil eine bessere Beweglichkeit als diejenigen mit dem inaktiven Lebensstil. „It can be concluded that age-related decay of mobility in the joints herein studied can be efficiently contrasted with an active style of life and that a training period can further improve the mobility of the lower limb" (ebd., S. 25). Aus den vorgenannten Untersuchungen wird deutlich, dass bezüglich des Einflusses der körperlichen Aktivität auf die Beweglichkeit in höherem Lebensalter keine einheitlichen Ergebnisse vorliegen. Um die Untersuchungsergebnisse korrekt einordnen zu können ist zu berücksichtigen, dass sowohl unterschiedliche Erhebungsinstrumente zur Messung der körperlichen Aktivität als auch unterschiedlich gemessene Gelenke eine Vergleichbarkeit solcher Studien einschränken.

\subsection{Biomechanische Grundlagen}

Nachfolgend werden biomechanische Parameter dargestellt, die während einer Muskeldehnung oder nach einem Dehntraining in vivo erhoben werden können. Dabei werden überblicksartig einzelne Anpassungseffekte beschrieben. Eine ausführliche Darstellung von Anpassungseffekten erfolgt anschließend in Kapitel 3 auf Grundlage eines systematischen Reviews. 
Sollen biomechanische Anpassungserscheinungen nach oder während einer Muskeldehnung erfasst werden, so wird zumeist die Wadenmuskulatur sowie die ischiocrurale Muskulatur getestet. Weitere aber wesentlich weniger bevorzugte Muskeln sind die Kniestreck- und die Hüftbeugemuskulatur. Der Vorteil der genannten Muskeln liegt darin, dass diese sich ohne Massenhemmung anderer Weichteile oder der Limitierung eines Knochen-Gelenksystems maximal dehnen lassen. Das bedeutet, dass der limitierende Faktor zur Erreichung einer maximalen Beweglichkeit und somit auch Muskeldehnfähigkeit nur durch eine Muskelhemmung besteht. Klee und Wiemann (2005, S. 24) beschreiben als Muskelhemmung „... wenn die Muskeln aufgrund ihrer passiven Spannung die Gelenkbewegung stoppen. Dies tritt in der Regel nur dort auf, wo Muskeln mehr als ein Gelenk überspannen“. Notwendig zur Erfassung biomechanischer Anpassungen sind darüber hinaus adäquate Messsysteme, die primär für die Wadenmuskulatur (vgl. Buckner et al., 2015; Gajdosik, Allred, Gabbert, \& Sonsteng, 2007) und die ischiocrurale Muskulatur (vgl. Glück et al., 2002; Magnusson, Simonsen, Aagaard, Sorensen, \& Kjaer, 1996; Schönthaler et al., 1998; Wiemann, 1991) vorhanden sind. Darüber hinaus existieren auch Messapparaturen für die Kniestreckmuskulatur (vgl. Klee, Jöllenbeck, \& Wiemann, 2000).

\subsubsection{Beweglichkeit}

Durch ein Dehntraining wird die Beweglichkeit verbessert. Diese Erkenntnis gilt als gesichert (Decoster et al., 2005; Medeiros, Cini, Sbruzzi, \& Lima, 2016). Die Beweglichkeit stellt dabei „... die maximal mögliche Amplitude in einem Gelenk dar" (Freiwald, 2009, S. 18) und wird in Winkelgraden [ $\left.{ }^{\circ}\right]$ angegeben. Sowohl nach kurz- als auch langzeitigen Dehnungsinterventionen wird eine Verbesserung der Beweglichkeit erreicht. In den nachfolgend dargestellten Studien ist zu erkennen, dass dieser Effekt unabhängig von einer bestimmten Dehnmethode ist. Covert, Alexander, Petronis und Davis (2010) konnten im Rahmen eine Metaanalyse zeigen, dass sowohl die statische als auch die dynamische Dehnmethode an der ischiocruralen Muskulatur zu einer Verbesserung der Beweglichkeit führt. Jedoch war die statische Dehnmethode der dynamischen überlegen. Wydra, Bös und Karisch (1991) verglichen drei Dehnmethoden (sta- 
tisch, dynamisch, postisometrisch) an der ischiocruralen Muskulatur über einen Zeitraum von zwei Wochen. Alle drei Dehnmethoden verbesserten die aktive und passive Beweglichkeit. Allerdings war hier die dynamische Dehnmethode den anderen beiden Methoden signifikant überlegen. Borges, Medeiros, Minotto und Lima (2017) verglichen in einer Metaanalyse den Unterschied zwischen der statischen Dehnmethode und der PNF-Methode an der ischiocruralen Muskulatur hinsichtlich einer Verbesserung der Beweglichkeit. Es wurde kein Unterschied zwischen den beiden Dehnmethoden festgestellt.

\subsubsection{Dehnungsspannung}

Nach Ryan et al. (2014) korreliert die maximale Beweglichkeit signifikant mit der maximalen Dehnungsspannung. Sie kann in eine maximale und submaximale Dehnungsspannung unterteilt werden (Klee, 2003, S. 4). Die Dehnungsspannung ist „... der Widerstand, den der Muskel aufgrund seiner Materialeigenschaften (plastische, elastische und visköse Eigenschaften) und einer eventuellen muskulären Aktivierung einer Längenveränderung entgegensetzt“ (Schönthaler et al., 1998, S. 225). Die submaximale Dehnungsspannung hingegen lässt sich definieren, als die Dehnungsspannung beim Erreichen eines submaximalen Gelenkwinkels. Die maximale Dehnungsspannung kann als mechanische Spannung der gedehnten Muskulatur in dem maximal zu erreichenden Gelenkwinkel definiert werden. Die submaximale Dehnungsspannung wird herangezogen, um zu überprüfen, ob sich die Ruhedehnungsspannung nach einer Dehnungsintervention herabsetzen lässt. Die Dehnungsspannung kann als Kraft [N] oder als Drehmoment [Nm] erfasst werden. Konrad und Tilp (2014) untersuchten die submaximale Dehnungsspannung nach einem sechswöchigen Dehntraining der Wadenmuskulatur und konnten keine signifikante Veränderung zwischen Pre- und Post-Test feststellen. Wiemann (1994) zeigte, dass es zwischen zwei einzelnen Dehnungen zu einer akuten Reduzierung der submaximalen Dehnungsspannung kommt. Allerdings wird hier eher ein Gewöhnungsbeziehungsweise Aufwärmeffekt vermutet, der keinen besonderen Einfluss auf die Muskeldehnfähigkeit hat (vgl. Knudson, 2006). 
Die während einer Muskeldehnung entstehende Dehnungsspannungskurve hat einen exponentiell ansteigenden Verlauf mit drei charakteristischen Eigenschaften (Abb. 12).

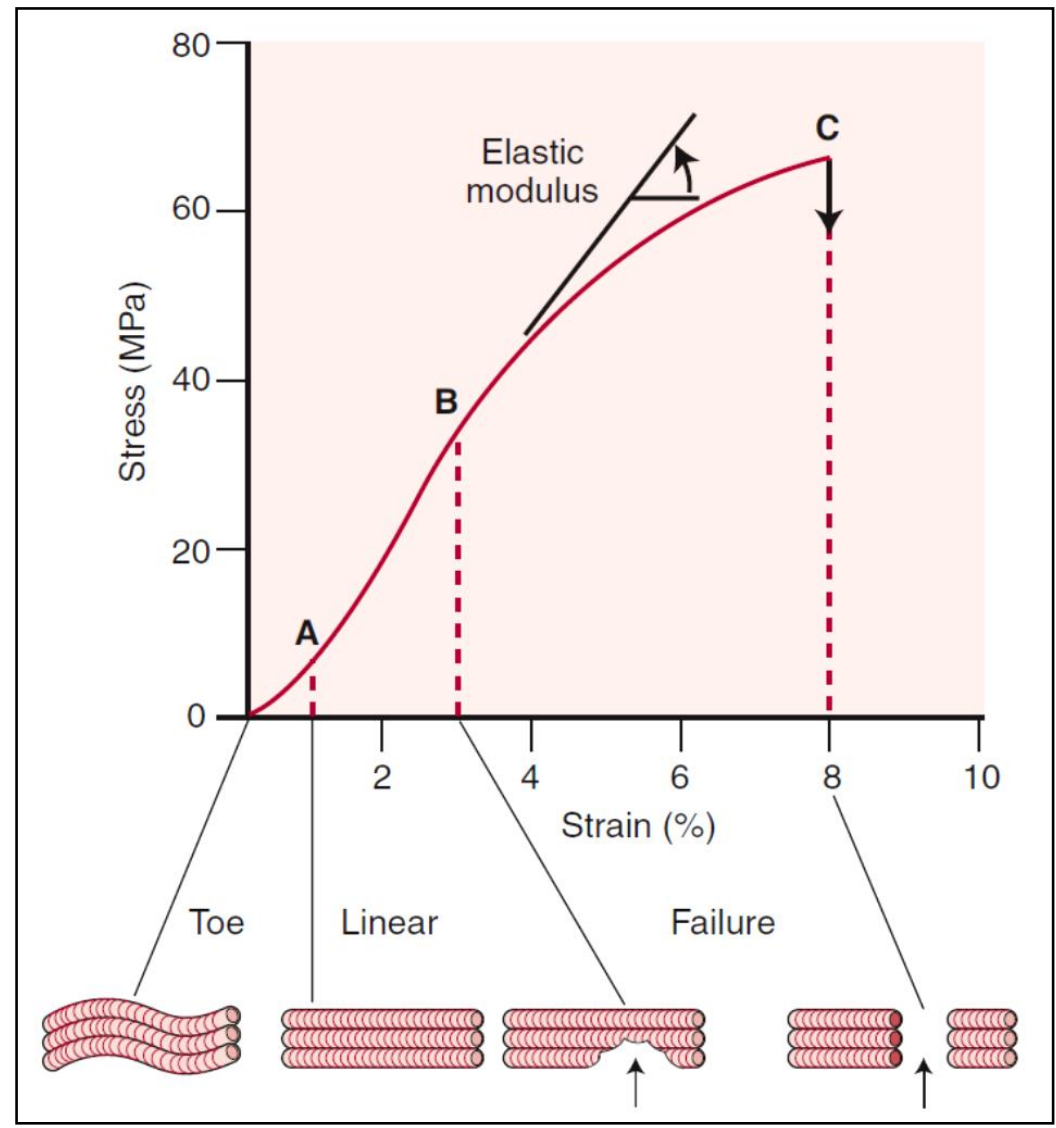

Abb. 12 Exemplarische Darstellung einer Dehnungsspannungskurve ( $A=$ Entfaltung der wellenförmigen Kollagenfasern, $B$ = elastischer Bereich, $C=$ Zerstörung des $G e-$ webes) (Chleboun, 2005, S. 86)

Während einer Muskeldehnung haben sowohl die kontraktilen als auch die parallel- und seriell-elastischen Komponenten einen Einfluss auf die Dehnungsspannung. Ein Muskel kann mit wenig Kraft beziehungsweise geringer Dehnungsspannung zunächst einfach gedehnt werden. Je weiter ein Muskel gedehnt wird, desto mehr steigt die Dehnungsspannung an. Lindel (2010, S. 14) beschreibt die Dehnungsspannung als Dehnungswiderstand. Ein steiler Anstieg des Dehnungswiderstandes wird primär durch die bindegewebigen Strukturen bestimmt. Im ersten Abschnitt der Dehnungsspannungskurve (Abb. 12, A) 
kommt es bei sehr geringer Dehnung zu einer Entfaltung der wellenförmigen Kollagenfasern (Dourte, Kuntz, \& Soslowsky, 2008; Hansen, Weiss, \& Barton, 2002). Der zweite Abschnitt wird als elastische Region beschrieben, in der die Dehnungsspannungskurve linear verläuft (Abb. 12, B). Es kommt bei Einwirkung einer Dehnung zu einer reversiblen Deformation des Gewebes. Nachdem die Dehnung nachlässt, kehrt das Gewebe unmittelbar in seine Ursprungsposition zurück (Curwin, 2005, S. 83). Der dritte Abschnitt der Dehnungsspannungskurve gilt als plastische Region (Abb. 12, C). Diese ergibt sich aus einer immer höheren Dehnung, die über die elastischen Eigenschaften der Strukturen hinausgeht. Es kommt zur weiteren Deformation des Gewebes bis hin zur Belastungsgrenze, an der das Gewebe irreversibel zerstört wird (Curwin, 2005, S. 83). In Anlehnung an Wang, Mc Carter, Wright, Beverly und RamirezMitchell (1993) kommt es bei einer maximalen Dehnung zu einem Abflachen der Dehnungsspannungskurve, dem sogenannten Yield-Point (Abb. 13).

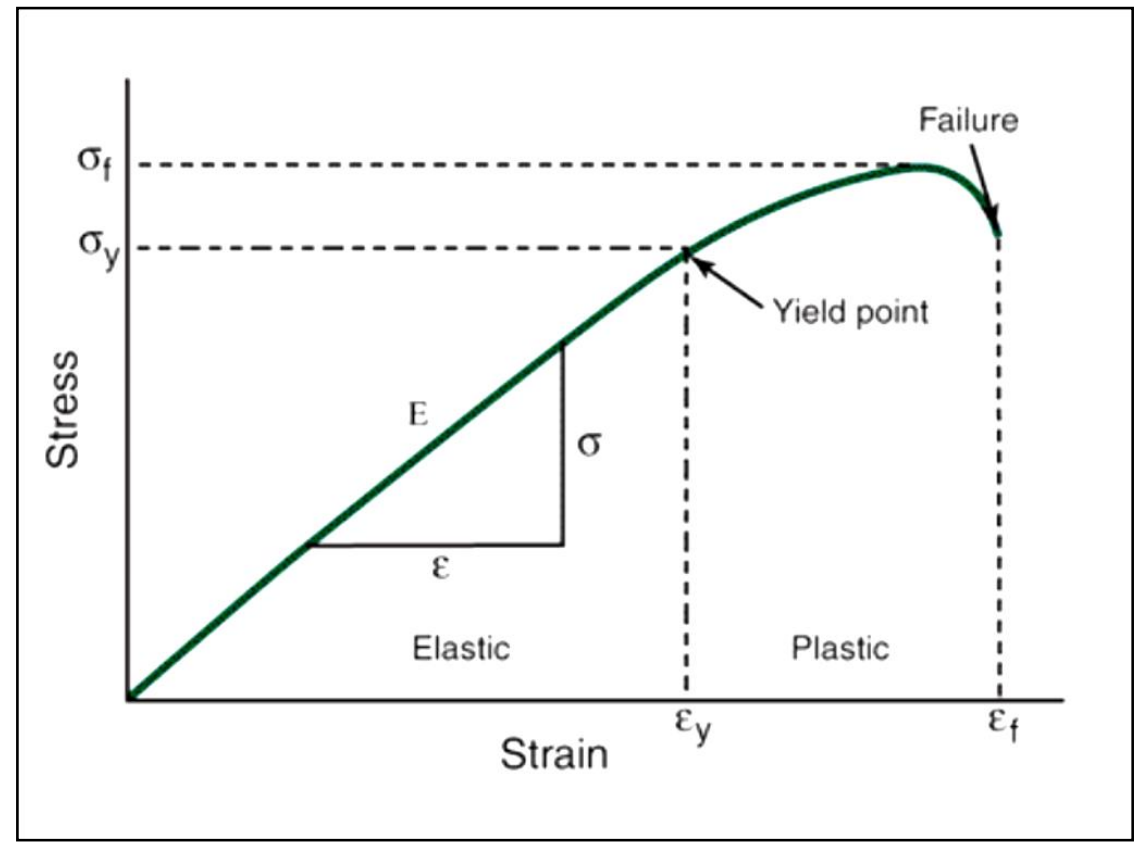

Abb. 13 Exemplarische Dehnungsspannungskurve mit Darstellung der elastischen und plastischen Region sowie dem Yield-Point ( $E$ = Ableitung der Stiffness) (Hamill \& Knutzen, 2009, S. 29) 


\subsubsection{Viskoelastische Eigenschaften}

Bezüglich der Muskeldehnfähigkeit lassen sich viskoelastische Eigenschaften der Muskulatur abbilden. Aufgrund der elastischen Eigenschaften, ähnlich einer mechanischen Feder, sowie der viskosen Eigenschaften, ähnlich eines Dämpfers, wird die Muskulatur als viskoelastisch charakterisiert (Butler et al., 1978, S. 134; Hung \& Mow, 2012, S. 69). Die Viskoelastizität wird von Klee (2003, S. 301) beschrieben a) als plastische Eigenschaften der Muskulatur, wie der Längenzunahme (Creeping) bei einer Dehnung mit gleichbleibender Dehnungsspannung, b) durch eine Spannungsrelaxation bei einer Dehnung bis zu einer gleichbleibenden Länge, c) durch eine Hystereseschleife, d) durch eine Zunahme der Dehnungsspannung mit zunehmender Dehnungsgeschwindigkeit.

\subsubsection{Creeping}

Wird die Muskulatur mit einer gleichbleibenden Spannung gedehnt und die Dehnungsspannung über einen gewissen Zeitraum beibehalten, so kommt es zu einem sogenannten Creeping-Phänomen. Hierbei kommt es bei gleichbleibender Dehnungsspannung zu einer Längenzunahme der Muskulatur (Abb. 14).

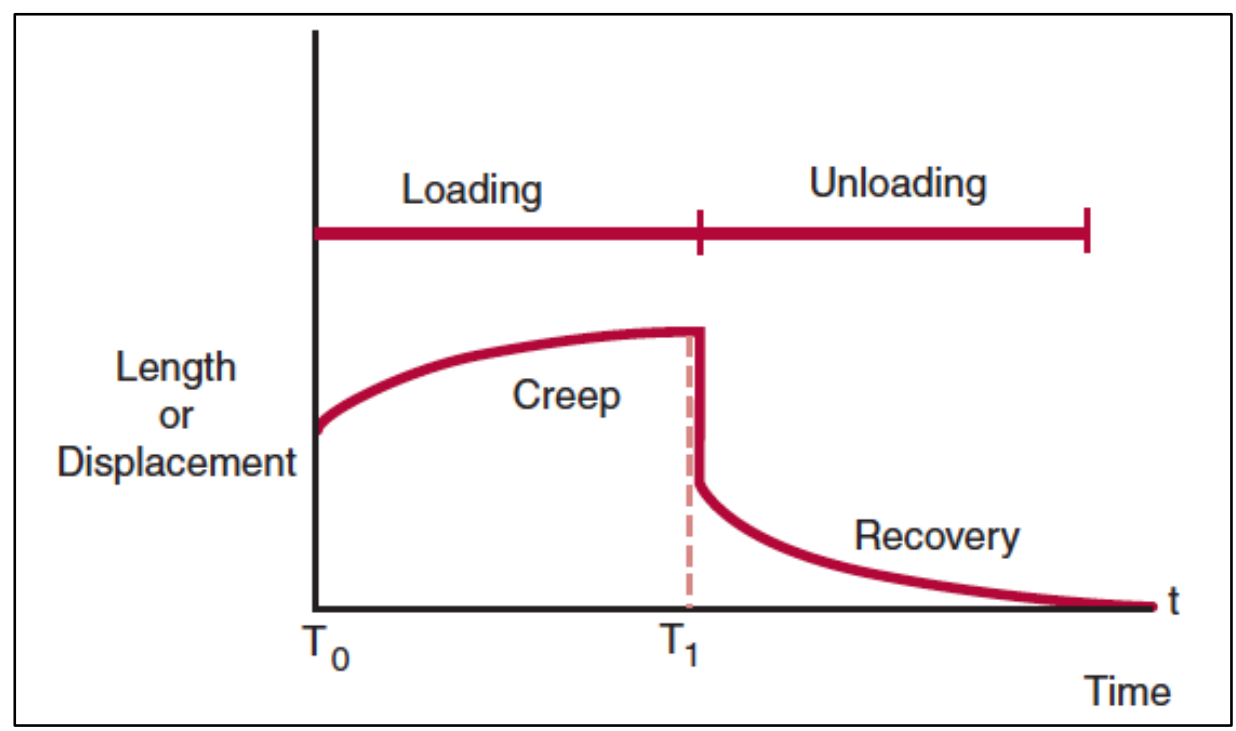

Abb. 14 Exemplarische Darstellung eines Creeping (Curwin, 2005, S. 88) 
Ryan, Herda, Costa, Walter und Cramer (2012) untersuchten den CreepingEffekt an der Wadenmuskulatur während einer viermaligen 30-sekündigen Dehnung bei einer konstanten Dehnungsspannung. In allen vier Dehnungen vergrößerte sich der Gelenkwinkel, wobei $84 \%$ der gesamten Veränderung in den ersten 15 bis 20 Sekunden einer Dehnung stattfand. Die Veränderung der Gelenkwinkel war zwischen der ersten und der zweiten Dehnung signifikant. Ab der dritten Dehnung resultierten keine signifikanten Veränderungen.

\subsubsection{Spannungsrelaxation}

Eine Spannungsrelaxation tritt auf, wenn die Muskulatur bei einer bestimmten Länge (Gelenkwinkel) gedehnt wird und in dieser Position verharrt. Hierbei wird die Dehnungsspannung allmählich nachlassen (Abb. 15).

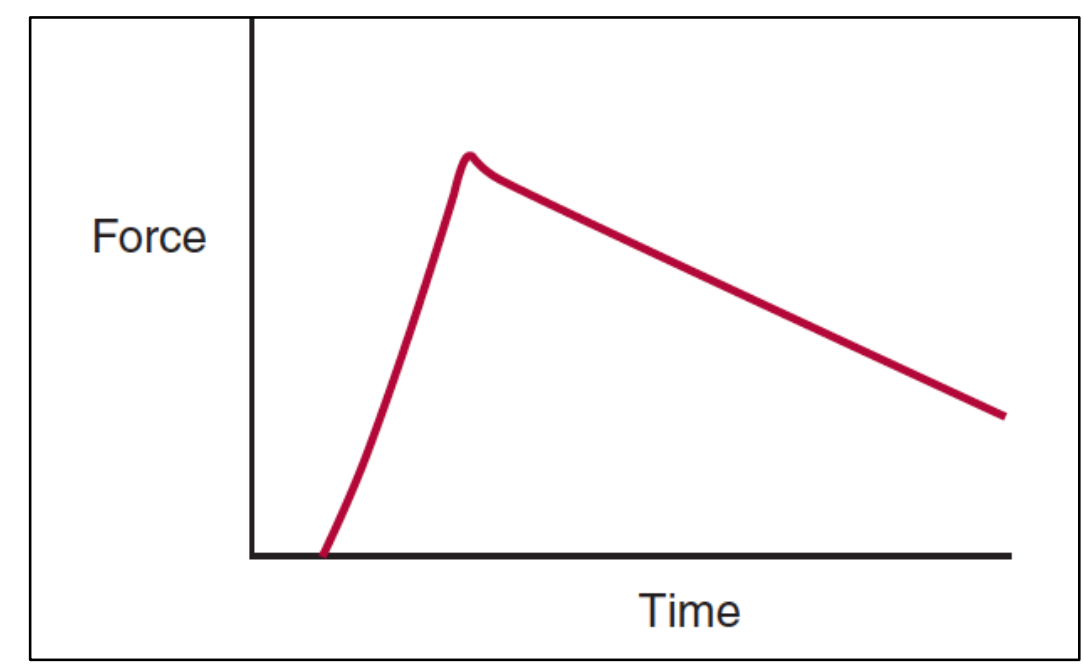

Abb. 15 Exemplarische Darstellung einer Spannungsrelaxation (Curwin, 2005, S. 88)

Magnusson, Aagaard und Nielson (2000) zeigten nach einer dreimaligen 45sekündigen Dehnung der ischiocruralen Muskulatur eine Spannungsrelaxation in der ersten Dehnung von $20 \%$ und in der dritten Dehnung von $18 \%$. Es konnte kein signifikanter Unterschied zwischen der ersten und dritten Dehnung bezüglich der Spannungsrelaxation festgestellt werden. Nach einem zehnwöchigen statischen Dehntraining an der ischiocruralen Muskulatur untersuchten 
Peixoto et al. (2015) die Spannungsrelaxation an einer sechsmaligen 30sekündigen Dehnung. An einer vorgegebenen Dehnungsspannung reduzierte sich die relative Spannungsrelaxation zwischen Pre- (25\%) zu Post-Test (21\%) signifikant. Des Weiteren zeigte sich eine signifikante Verringerung der Spannungsrelaxation zwischen der zweiten und dritten Dehnung. Zwischen der vierten und sechsten Dehnung war der Unterschied nicht mehr signifikant. Aus diesem Grund geben Peixoto et al. (2015, S. 761) an, dass ein Dehntraining mit zwei bis vier Sätzen durchgeführt werden sollte.

Magnusson et al. (1996) untersuchten akute viskoelastische Anpassungen an der ischiocruralen Muskulatur nach einer fünfmaligen 90-sekündigen Dehnung. Zwischen der ersten und fünften Dehnung reduzierte sich die Dehnungsspannung signifikant über die 90-sekündige Dehnung. Nach einer Wiederholungsmessung eine Stunde später war dieser Effekt reversibel. Es konnte kein Unterschied zwischen der ersten Dehnung und der Wiederholungsmessung festgestellt werden.

\subsubsection{Hysterese}

Die Muskulatur weist unterschiedliche Dehnungsspannungskurven bei der Dehnung und der Entdehnung auf, die als Hystereseschleife beschrieben wird (Weppler \& Magnusson, 2010, S. 440). Die Kurve während der Entdehnung (Entlastung des Gewebes) weist eine insgesamt geringere Dehnungsspannung auf (Abb. 16). 


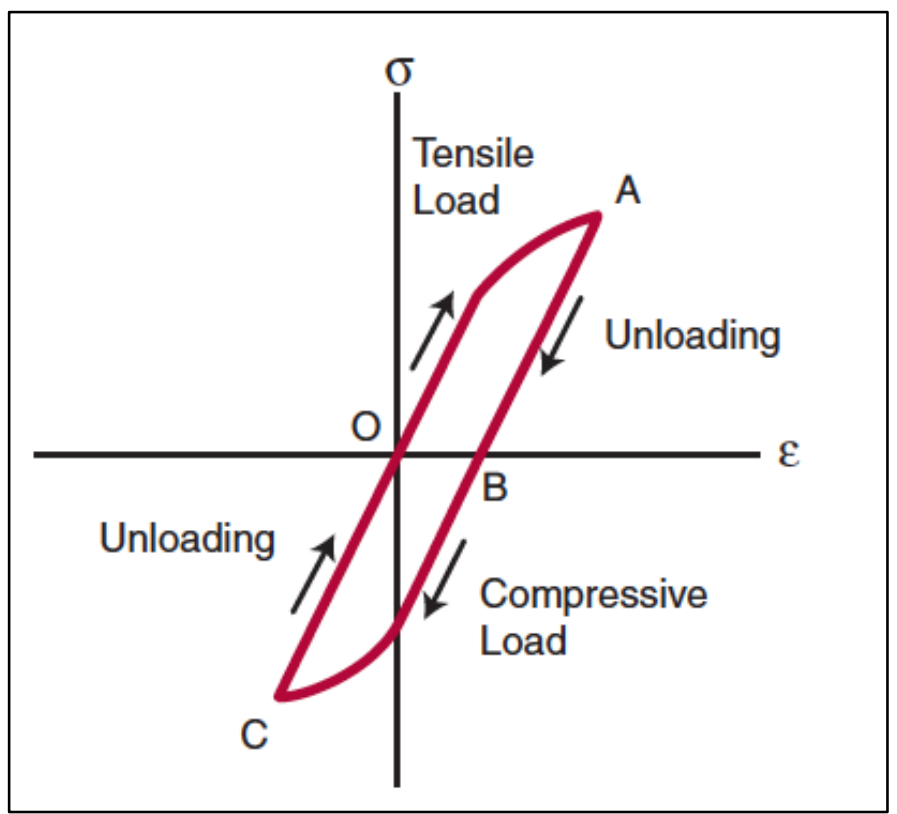

Abb. 16 Exemplarische Darstellung einer Hystereseschleife (die Dehnung beginnt bei O; die Dehnungsspannung verläuft über $A$ - B - C) (Curwin, 2005, S. 88)

Kubo, Kanehisa und Fukunaga (2002) untersuchten die Hysterese nach einem dreiwöchigen statischen Dehntraining an der Wadenmuskulatur. Die Probanden dehnten sich zweimal täglich (morgens und abends). Pro Dehneinheit sollte die Muskulatur fünfmal für jeweils 35 Sekunden gedehnt werden. Es konnte festgestellt werden, dass sich die Fläche unter der Dehnungs- und Entdehnungskurve als Hystereseschleife um $37 \%$ signifikant verringerte. Ebenso verringerte sich die Hysterese nach einer einmal durchgeführten zehnminütigen statischen Dehnung der Wadenmuskulatur (Kubo, Kanehisa, Kawakami, \& Fukunaga, 2001). Nordez, McNair, Casari und Cornu (2008) konnten ebenfalls eine Reduzierung der Hysterese nach einer sechsmaligen 30 -sekündigen Dehnung der ischiocruralen Muskulatur beobachten.

\subsubsection{Dehnungsgeschwindigkeit}

Je nach Geschwindigkeit der Muskeldehnung kommt es zu unterschiedlich steilen Dehnungsspannungskurven. Wird ein Muskel relativ schnell gedehnt, so wird eine höhere Dehnungsspannung erreicht (Abb. 17). Die daran anschlie- 
Bende Spannungsrelaxation wird nach Curwin (2005, S. 88) größer ausfallen als bei einer Dehnung mit geringerer Geschwindigkeit.

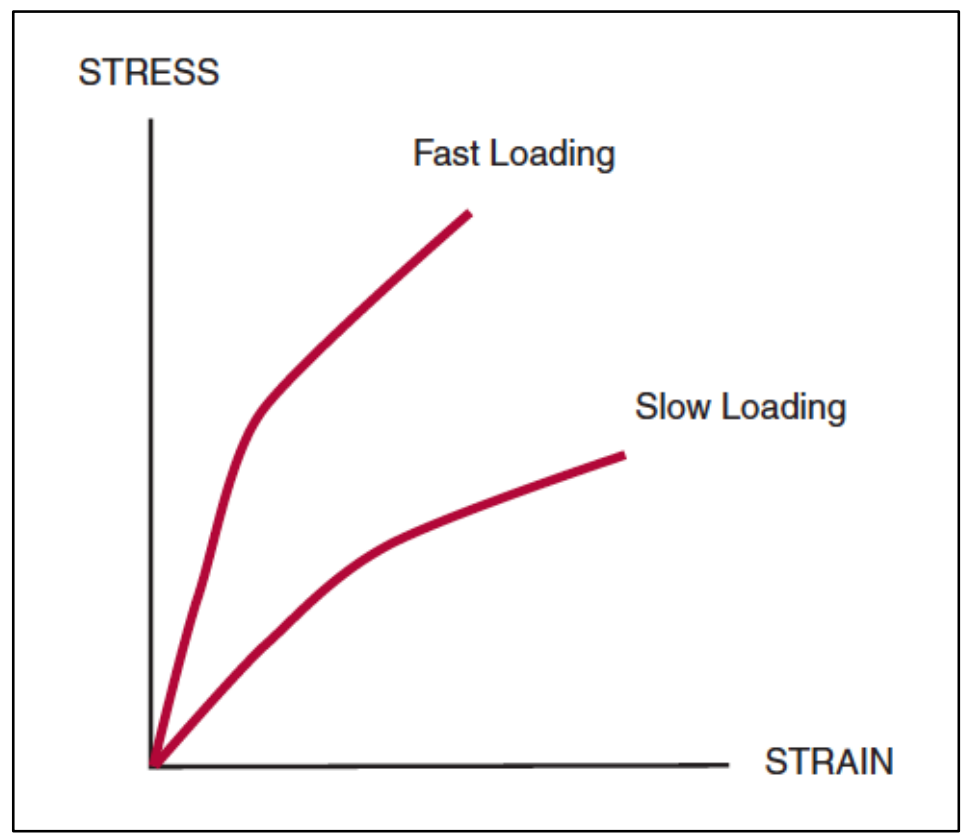

Abb. 17 Exemplarische Darstellung von zwei Spannungskurven bei hoher Dehnungsgeschwindigkeit (links) und bei geringer Dehnungsgeschwindigkeit (rechts) (Curwin, 2005, S. 88)

McNair, Hewson, Dombrowski und Stanley (2002) untersuchten zwei Dehnungsgeschwindigkeiten (5 deg/s vs. $25 \mathrm{deg} / \mathrm{s}$ ) während einer Dehnung der Wadenmuskulatur. Die Dehnung wurde bei $80 \%$ der individuellen maximalen Beweglichkeit für zwei Minuten gehalten. Bei höherer Dehnungsgeschwindigkeit $(25 \mathrm{deg} / \mathrm{s})$ resultierte eine signifikant größere maximale Dehnungsspannung als bei niedriger Geschwindigkeit ( $5 \mathrm{deg} / \mathrm{s}$ ). Zudem lag die Spannungsrelaxation bei der höheren Dehnungsgeschwindigkeit bei $21 \%$ und bei der geringeren Geschwindigkeit bei $13 \%$.

\subsubsection{Stiffness}

In Abhängigkeit der Dehnungsspannung sowie der Beweglichkeit lassen sich die Stiffness (Abb. 13, E) sowie die Fläche unterhalb der Dehnungsspannungskurve (Kapitel 2.3.5) als elastische Parameter der Muskeldehnfähigkeit ableiten 
(LaRoche \& Connolly, 2006; Magnusson, 1998; Reid \& McNair, 2004). Die Stiffness wird nach Curwin (2005, S. 85) als der Widerstand gegen eine äußere Kraft verstanden. Die Stiffness beschreibt demnach die elastische muskelphysiologische Response auf eine Muskeldehnung (Alter, 2004, S. 55). Nach Hutton (1994, S. 43) wird die Stiffness definiert „... als die Änderung der auf den Muskel einwirkenden Kraft dividiert durch die Änderung der Muskellänge“. Die Beweglichkeit ist bezüglich dieser Definition äquivalent zur Muskellänge. Somit ist die Stiffness die Änderung der Dehnungsspannung [N] oder [Nm] dividiert durch die Beweglichkeit $\left[^{\circ}\right]$ und wird angegeben als $\left[\mathrm{N} /{ }^{\circ}\right]$ oder $\left[\mathrm{Nm} /{ }^{\circ}\right]$. Die Problematik bei deren Erhebung ist, dass die Stiffness in der Literatur unterschiedlich definiert wird und somit ein Vergleich von Studien erschwert (Latash \& Zatsiorsky, 1993). Beispielsweise untersuchten La Roche und Connolly (2006) die Stiffness anhand eines vorher festgelegten Winkelbereiches und konnten keine Veränderung nach einem vierwöchigen Dehntraining zeigen. Reid und McNair (2004) untersuchten die Stiffness anhand der letzten $10 \%$ der Dehnungsspannungskurve. Auch hier konnte keine Veränderung der Stiffness nach einem sechswöchigen Dehntraining festgestellt werden.

\subsubsection{Fläche unterhalb der Dehnungsspannungskurve}

Die Fläche unterhalb der Dehnungsspannungskurve wird als Integral angegeben und repräsentiert in Anlehnung an Magnusson (1998) die absorbierte passive-elastische Energie (Abb. 18). 


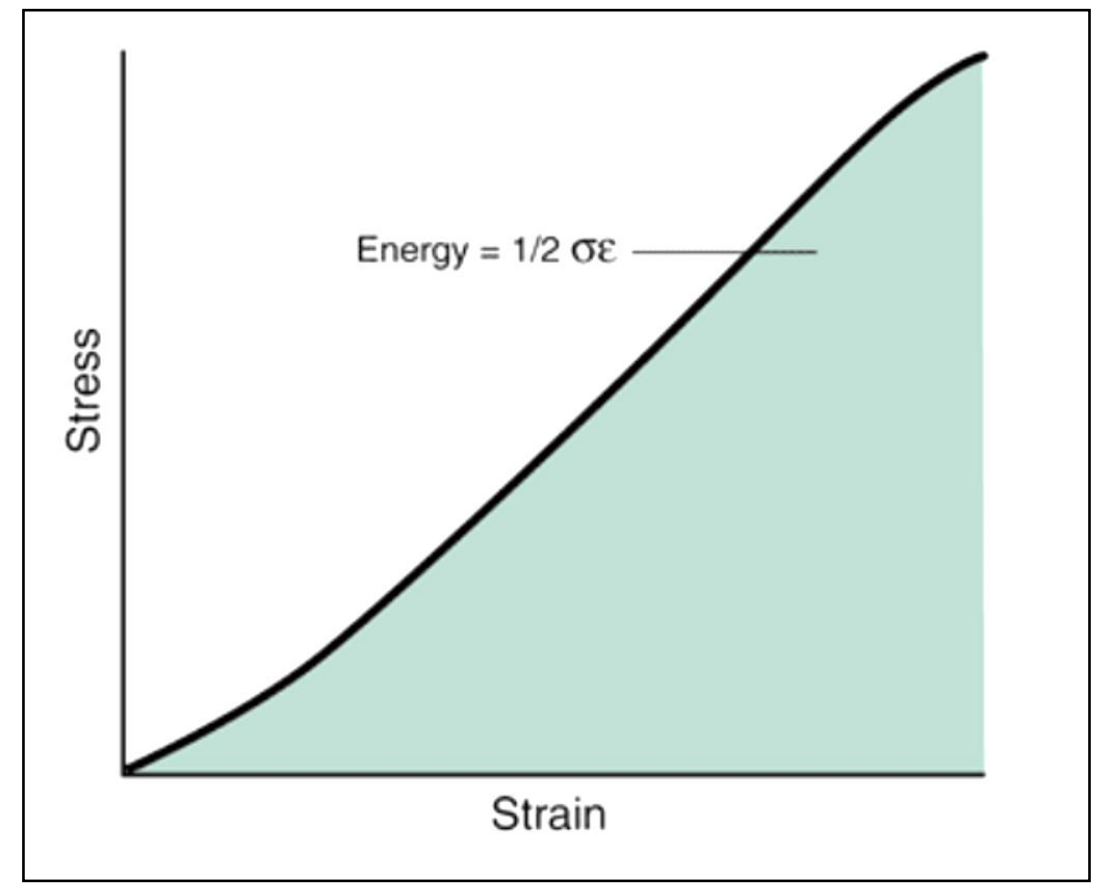

Abb. 18 Exemplarische Darstellung der Fläche unterhalb der Dehnungsspannungskurve (Hamill \& Knutzen, 2009, S. 30)

Zur Berechnung der absorbierten passiven-elastischen Energie wird die Beweglichkeit $\left[{ }^{\circ}\right]$ mit der Dehnungsspannung $[\mathrm{N}]$ oder $[\mathrm{Nm}]$ multipliziert. Angegeben wird die passive-elastische Energie als $\left[{ }^{\circ} \mathrm{N}\right]$ oder [ $\left.{ }^{\circ} \mathrm{Nm}\right]$. Die Stiffness sowie die Fläche unterhalb der Dehnungsspannungskurve ist direkt abhängig von der maximal erreichten Beweglichkeit sowie der Dehnungsspannung. Demnach werden unterschiedliche Ergebnisse erzielt, wenn eine Muskeldehnung bis zu einer maximalen oder nur bis zu einer submaximalen Dehnungsspannung und somit einer mehr oder weniger großen Bewegungsreichweite erfolgt. Dieses Dilemma wird bei Magnusson (1998, S. 72) wie folgt beschrieben: „... for a given joint angle, tight subjects display greater stiffness and therefore more energy will be absorbed by the tissue. On the other hand, at a maximal joint angle normal subjects achieve a greater maximal torque and maximal stiffness and can therefore absorb more energy at this point than tight subjects". Aufgrund der Tatsache, dass sich sowohl die Beweglichkeit als auch die Dehnungsspannung nach einer Dehnungsintervention verändert (Chagas \& Schmidtbleicher, 2004; Gajdosik, 1991), wird sich auch die Fläche unterhalb der Dehnungsspannungskurve entsprechend verändern. Die Muskulatur wird also bei einer 
Verbesserung der Beweglichkeit und der Dehnungsspannung auch mehr passive-elastische Energie absorbieren können.

\subsection{Zusammenfassung}

Die Einleitung diente einer Einführung in die Thematik des Dehntrainings. Es wurden zunächst die wichtigsten Begriffe hinsichtlich eines Dehntrainings sowie der Beweglichkeit definiert. Ein Dehntraining wurde demnach als ein langzeitiges Dehnprogramm beschrieben mit dem primären Ziel, die Beweglichkeit zu verbessern. Es existieren keine allgemeingültigen Handlungsempfehlungen zur Durchführung eines Dehntrainings. Dies liegt an der Durchführung von Trainingsstudien mit unterschiedlichsten Belastungsparametern, die teilweise uneinheitlich oder aber auch unvollständig angegeben wurden. Eine weitere Problematik besteht in der nur schwierig zu quantifizierbaren Angabe der Trainingsintensität.

Zu den Einflussfaktoren auf die Beweglichkeit wurden neben dem Geschlecht, der Tageszeit, der Psyche, der Temperatur und dem Lebensalter auch anatomische und neurophysiologische Einflussfaktoren beschrieben.

Die Beweglichkeit ist abhängig von der Gelenkigkeit, der Dehnfähigkeit der Muskulatur sowie dem Bindegewebe. Im Rahmen eines Dehntrainings wird eine anatomische Struktur niemals einzeln gedehnt. Man spricht dann genau genommen von einer Dehnung des Muskel-Faszien-Sehnen-Komplexes, welcher aus kontraktilen sowie seriell- und parallel-elastischen Elementen besteht. Hierbei spielt das Titinfilament als parallel-elastisches Element eine bedeutende Rolle bezüglich der Ruhespannung und der Muskeldehnfähigkeit. Weiterhin besteht die Muskulatur zu ca. 30 \% aus Bindegewebe, sodass den Faszien, ebenfalls ein parallel-elastisches Element, sowie den kollagenen Fasern eine bedeutende Rolle bei der Erreichung einer maximalen Beweglichkeit beziehungsweise der Muskeldehnfähigkeit zukommt. Wird ein Muskel gedehnt, so kommt es zu einer exponentiell ansteigenden Dehnungsspannungskurve. An- 
hand dieser Dehnungsspannungskurve lassen sich biomechanische Parameter ableiten. Dazu gehören die Dehnungsspannung, die Stiffness, die Fläche unterhalb der Dehnungsspannungskurve sowie viskoelastische Parameter.

Neben den anatomischen Einflussfaktoren haben außerdem neurophysiologische Einflussfaktoren eine Wirkung auf die Beweglichkeit. Dementsprechend wurden die beiden sensorischen Rezeptoren Muskelspindeln und GolgiSehnenorgane ausführlich beschrieben. Die Muskelspindeln wurden als primärer Dehnungsrezeptor identifiziert. Wird ein Muskel gedehnt, so kann es ausgehend von der Aktivierung der beiden sensorischen Rezeptoren zu spezifischen Aktionspotentialen in der Muskulatur kommen. Diese können über eine Elektromyographie erfasst werden.

Im Rahmen der neurophysiologischen Einflussfaktoren wurde weiterhin eine neuromuskuläre Dysbalance beschrieben. Aufgrund von pathologischen Störungen, wie beispielsweise einer Arthrose, oder aufgrund von nichtpathologischen Störungen, wie beispielsweise Fehlhaltungen im Alltag, kann eine neuromuskuläre Dysbalance die Beweglichkeit negativ beeinflussen.

Es ist festzuhalten, dass mit zunehmendem Lebensalter die Beweglichkeit, sowohl für Männer als auch für Frauen, stetig abnimmt. Frauen zeigen allerdings bessere Beweglichkeitsmaße als Männer. Die Gründe für die Verringerung der Beweglichkeit bestehen in degenerativen Veränderungen des Gelenksystems. Außerdem nimmt mit zunehmendem Lebensalter die Muskelmasse ab und die Einlagerung von kollagenem Bindegewebe in die Muskulatur nimmt zu. Daher kommt es insgesamt zu einer Verminderung der Dehnbarkeit und Elastizität von Muskel- und Bindegewebe, was zu einer altersbedingten Zunahme der Stiffness führt. 


\section{Altersspezifische Anpassungen an ein Dehntraining: Ein systematischer Review}

Nachdem im vorherigen einleitenden Kapitel grundlegende Aspekte bezüglich des Dehntrainings sowie der Einflussfaktoren auf die Beweglichkeit und deren Veränderung im Alternsgang beschrieben wurden, wird nachfolgend der aktuelle Forschungsstand zu den Anpassungserscheinungen an ein Dehntraining bei jüngeren und älteren Erwachsenen dargestellt. Dabei wird nicht das Ziel verfolgt, Aussagen über die Effektivität eines Dehntrainings auf physiologischer, morphologischer oder struktureller Art zu treffen, sondern primär die zwei wesentlichen biomechanischen Parameter Beweglichkeit und Dehnungsspannung zur Beurteilung eines Dehntrainings altersspezifisch zu vergleichen. Um den aktuellen Forschungsstand zu den Anpassungserscheinungen an ein Dehntraining darzulegen und darüber hinaus diese Erkenntnisse zwischen jüngeren und älteren Erwachsenen zu vergleichen, wurde eine systematische Literaturrecherche durchgeführt ${ }^{1}$. Die Ergebnisse der Literaturrecherche wurden zudem einer metaanalytischen Betrachtung unterzogen.

\subsection{Methodik}

Die Literaturrecherche erfolgte über die Internetdatenbanken Pubmed, Cochrane, Web of Science sowie über das BISp-Recherchesystem Sport (Bundesinstitut für Sportwissenschaft) mit den Datenbanken SPOFOR und SPOLIT. Die Recherche in den Datenbanken wurde durch zusätzliche Filter ergänzt (Tab. 2).

${ }^{1}$ Diese Untersuchung wurde unter dem Titel „Altersspezifische Anpassungen an ein Dehntraining. Eine metaanalytische Betrachtung." im German Journal of Exercise and Sport Research im Juli 2017 veröffentlicht (Haab, Martini, Baluktsian, \& Wydra, 2017). 
Tab. 2 Filter in den verwendeten Datenbanken

\begin{tabular}{|c|c|}
\hline Datenbank & Filter \\
\hline Pubmed & $\begin{array}{l}\text { - } \quad \text { clinical trial } \\
\text { - } \quad 01.01 .1990-31.10 .2015\end{array}$ \\
\hline Cochrane & $\begin{array}{ll}\text { - } & \text { trials } \\
\text { - } & 1990-2015 \\
\text { - } & \text { Title-Abstract-Keywords }\end{array}$ \\
\hline Web of Science & $\begin{array}{l}\text { - } \quad \text { clinical trial } \\
\text { - } 1990-2015\end{array}$ \\
\hline BISp-Recherchesystem & - $1990-2015$ \\
\hline
\end{tabular}

Bei den Deskriptoren handelte es sich zunächst um die allgemeinen Schlagwörter "stretch" und "stretching" beziehungsweise "dehnung“ und „dehnen“ mit und ohne dem Zusatz "muscle“ beziehungsweise „muskel“, die sodann über Boolesche Operatoren in Kombination mit einem entsprechenden Dehnparameter in die Suchmaske eingegeben wurden ${ }^{2}$. Nicht erwünschte Treffer wurden vorab in der Suchanfrage mit einem entsprechenden Filter versehen (Tab. 3). Es wurde in Anlehnung an Rustenbach (2003, S. 26) eine Vorabrecherche durchgeführt, um bezüglich einer multiplen Recherchestrategie eine möglichst hohe Identifikation potentieller Quellen zu gewährleisten sowie mit einer möglichst hohen Präzision die Primärstudien einzugrenzen und irrelevante Treffer auszuschließen.

2 Beispiel einer Suchanfrage über Pubmed: (stretch OR "muscle stretch" OR stretching OR "muscle stretching") AND (rom OR "range of motion" OR "joint range") NOT strength NOT balance NOT osteoarthritis NOT contracture NOT parkinson NOT "multiple sclerosis" NOT fibromyalgia NOT yoga NOT "tai chi" NOT injury NOT surgery 
Tab. 3 Deskriptoren zur Literaturrecherche

\begin{tabular}{|c|c|c|}
\hline Allgemein & Dehnparameter (AND / OR) & Filter (NOT) \\
\hline stretch & rom & strength \\
\hline stretching & „range of motion“ & balance \\
\hline „muscle stretch“ & „joint range” & osteoarthritis \\
\hline „muscle stretching“ & viscoelastic* & contracture \\
\hline dehnung & „passive tension” & „multiple sclerosis” \\
\hline dehnen & „passive resistive tension” & fibromyalgia \\
\hline muskeldehnung & „passive torque” & „tai chi“ \\
\hline \multirow[t]{13}{*}{ muskeldehnen } & „passive resistive torque” & yoga \\
\hline & „passive force” & injury \\
\hline & „passive resistive force” & surgery \\
\hline & dehnfähigkeit & kraft $^{*}$ \\
\hline & dehnbarkeit & gleichgewicht* \\
\hline & beweglichkeit & arthrose \\
\hline & gelenkbeweglichkeit & osteoarthrose \\
\hline & bewegungsreichweite & kontraktur \\
\hline & viskoelast* $^{*}$ & parkinson \\
\hline & & „multiple sklerose” \\
\hline & & fibromyalgie \\
\hline & & verletzung \\
\hline & & operation \\
\hline
\end{tabular}

In Studien zum Dehntraining werden zumeist Anpassungserscheinungen der unteren Extremitäten erhoben. Aus diesem Grund beschränkt sich die Literaturrecherche auf Studien, die ein Dehntraining der Waden- und Hüftbeugemuskulatur sowie der Beinbeuge- und Beinstreckmuskulatur durchführten. Außerdem sollten Studienergebnisse mit trainingspraktischer Relevanz dargestellt werden. Das bedeutet, dass die Studien ein langzeitiges Dehnungsprogramm von mindestens zwei Wochen Dauer aufweisen sollten. Es wurden sowohl Studien 
mit als auch ohne Kontrollgruppe ausgewählt. Die Literaturrecherche beschränkte sich bewusst nicht auf kontrollierte randomisierte Studien (RCT), da dies zu einer Verzerrung der Primärbefunde sowie einer Einschränkung der Repräsentativität führen würde (Rustenbach, 2003, S. 55). Ausgeklammert wurden Studien, die neben einer Dehnungsintervention zusätzlich andere Treatments zur Verbesserung einer sportmotorischen Fähigkeit durchführten, da in diesem Fall der Treatmenteffekt nicht eindeutig auf die Dehnungsintervention zurückzuführen wäre. Als Primärstudien wurden Untersuchungen in englischer und deutscher Sprache aufgenommen, die folgende Kriterien erfüllten:

a) Publikationszeitraum zwischen 1990 und 10/2015,

b) Intervention durch Muskeldehnung im Pre-Post-Design von mindestens zwei Wochen,

c) Versuchspersonen ohne orthopädische Erkrankungen,

d) Versuchspersonen in einem Alter zwischen dem 18. und 90. Lebensjahr,

e) Angabe und Beschreibung der verwendeten Dehnmethode und dazugehörigen Belastungsparametern,

f) die abhängige Variable wurde operationalisiert über die passive Beweglichkeit und/oder die Dehnungsspannung,

g) das Treatment sollte ein Dehntraining einer Muskelgruppe der unteren Extremitäten beinhalten,

h) Angaben der statistischen Kennwerte zur Berechnung der Effektstärke.

Bei den Einschlusskriterien der Primärstudien ist anzumerken, dass es bei älteren Probanden sicherlich schwierig ist, geeignete Personen ohne degenerative Verschleißerkrankungen in eine Interventionsstudie zu integrieren. Es kann nicht ausgeschlossen werden, dass zumindest subtile orthopädische Erkrankungen vorliegen. Diese Problematik ist ebenfalls für die hier vorliegende Literaturrecherche relevant. Allerdings sollte durch die genannten Einschlusskriterien versucht werden, ein möglichst homogenes Kollektiv an Primärstudien zu erhalten. 
Die Vorgehensweise der Literaturrecherche orientierte sich an den Leitlinien des Cochrane Handbook for Systematic Reviews of Intervention (Higgins \& Green, 2008) und ist unterteilt in drei Schritte (Abb. 19). Im ersten Schritt wurde über die Schlagwortsuche Literatur aus den oben genannten Datenbanken recherchiert. Über die Überschriften wurden relevante Studien und gleichzeitig Duplikate herausgefiltert. Im zweiten Schritt wurden die Studien sowohl über die Überschriften als auch über die Abstracts weiter eingegrenzt. Im dritten Schritt erfolgte eine inhaltliche Betrachtung der noch verbleibenden Studien und bei nicht relevantem Inhalt wurden diese Studien nicht weiter analysiert. Um Publikationsverzerrungen zu vermeiden, fand in einem Zwischenschritt eine Auswertung der Literaturverzeichnisse der aus dem ersten Schritt hervorgebrachten Studien statt (Hand searching of references). Diese Auswertung wurde den Rechercheergebnissen im zweiten Schritt hinzugefügt.

Zur Literaturverwaltung wurde das Programm Citavi 4 (Swiss Academic Software $\mathrm{GmbH}$ ) verwendet.

\subsubsection{Abhängige Variablen}

Die Anpassungserscheinungen an ein Dehntraining wurden über die abhängigen Variablen maximale Beweglichkeit als Gelenkwinkel [ ${ }^{\circ}$ ] und/oder die maximale Dehnungsspannung als Kraft [N] oder als Drehmoment [Nm] operationalisiert.

\subsubsection{Bewertung der Primärstudien}

Die Bewertung der Primärstudien erfolgte anhand der PEDro-Skala (Maher, Sherrington, Herbert, Moseley, \& Elkins, 2003), die in Anlehnung an Young, Nix, Wholohan, Bradhurst und Reed (2013) sowie Law et al. (1998) modifiziert wurde. Die Primärstudien wurde von zwei unabhängig arbeitenden Personen bewertet ${ }^{3}$. Insgesamt wurden 14 Kriterien herangezogen, die die interne und externe Validität sowie statistische Informationen der Primärstudien beurteilten (Tab. 5). Zu den ursprünglichen 11 Items der PEDro-Skala prüften zwei zusätz-

${ }^{3}$ Die Primärstudien wurden in Zusammenarbeit mit einer Bachelorarbeit bewertet (Baluktsian, 2017) 
liche Items, ob die Outcome-Messungen als hinreichend reliabel und valide beschrieben wurden. Ein weiteres Item sollte klären, ob die Stichprobengröße begründet wurde. Hier wurde das Item mit „Ja“ beantwortet, wenn die Stichprobengröße a priori begründet wurde. Um alle anderen Kriterien mit „Ja“ zu beantworten, mussten die Angaben in der Studie explizit beschrieben worden sein. Ansonsten erfolgte eine Bewertung mit "Nein“. Diese Vorgehensweise sollte für eine konsistente Bewertung der Primärstudien sorgen.

\subsubsection{Datenextraktion}

Die Datenextraktion erfolgte nach einem a priori festgelegten Kodierschema durch zwei unabhängig arbeitende Personen ${ }^{4}$. Das Kodierschema ist in Tabelle 4 in der Übersicht dargestellt und orientierte sich an den Cochrane Guidelines (Higgins \& Green, 2008) sowie an Stock (1994) und Wortman (1994).

In den Primärstudien konnten die Dehnmethoden als passiv-statische, passivdynamische, aktiv-statische bzw. aktiv-dynamische Dehnung oder als PNFMethode dargestellt werden. Weiterhin wurden die Dehnmethoden noch in die Dehnungsform (Eigen- und Fremddehnung) unterteilt. Die erfassten Daten wurden zur weiteren Verarbeitung in die Tabellenkalkulationssoftware Excel (Microsoft Office, 2007) eingegeben und sodann in die Statistiksoftware SPSS (IBM Corp., Version 23) übertragen.

${ }^{4}$ Die Datenextraktion wurde in Zusammenarbeit mit einer Bachelorarbeit durchgeführt (Martini, 2016) 
Tab. 4 Kodierung und Studiendetails der Primärstudien

\begin{tabular}{|l|l|}
\hline \multicolumn{1}{|c|}{ Item } & \multicolumn{1}{c|}{ Beschreibung } \\
\hline Publikation & Autor, Publikationsjahr \\
\hline Stichprobe & Versuchsgruppen, Probandenanzahl, Altersangaben \\
\hline Treatment & $\begin{array}{l}\text { Untersuchungszeitraum, Dehnmethode, Dehnungsform, Muskulatur, Be- } \\
\text { lastungsparameter (Häufigkeit, Dehndauer, Wiederholungen, Intensi- } \\
\text { tätsangabe) }\end{array}$ \\
\hline Testdurchführung & $\begin{array}{l}\text { manuelle oder apparative Testung, Testübung, Dehnungsform, Intensi- } \\
\text { tätsangabe }\end{array}$ \\
\hline Statistik & Angaben zur Berechnung der Effektstärke \\
\hline
\end{tabular}

\subsubsection{Ergebnisdarstellung}

Im Rahmen der Datenextraktion erfolgte die Bewertung und Überprüfung der Beurteilerübereinstimmung kategorialer Items nach Spitzer, Cohen, Fleiss und Endicott (1967) durch die Ermittlung der Interraterreliabilität über Cohens Kappa (к). Die Interraterreliabilität bei kontinuierlichen Items wurde nach McGraw und Wong (1996) durch Intraklassenkorrelationen (ICC) überprüft. Eine anschließende Bestimmung der Gesamtreliabilität ( $\kappa_{\text {gesamt}}$ ) erfolgte durch eine Interraterreliabilität über den gewichteten Mittelwert aller erhobenen Koeffizienten (Rustenbach, 2003, S. 50). Die Kategorisierung der Beurteilerübereinstimmung sowie der Gesamtreliabilität orientierte sich an Landis und Koch (1977): schlechte Übereinstimmung < 0.0, geringe Übereinstimmung $0.0-0.2$, ausreichende Übereinstimmung $0.21-0.4$, mittelmäßige Übereinstimmung $0.41-0.6$, erhebliche Übereinstimmung $0.61-0.8$, nahezu vollständige Übereinstimmung 0.81 - 1.0. Die Berechnung der Beurteilerübereinstimmung erfolgte mit der Statistiksoftware SPSS (IBM Corp., Version 23). 
Die Berechnung der Effektstärke (ES) im Rahmen der metaanalytischen Betrachtung erfolgte nach Hedges und Olkin (1985, S. 79) über die gepoolte Standardabweichung. Die Effektstärke wurde bestimmt anhand der primären abhängigen Variablen maximale Dehnungsspannung sowie der maximalen $\mathrm{Be}$ weglichkeit. Zur Überprüfung der Effektstärken zwischen jüngeren und älteren Erwachsenen wurden die Probanden aus den Primärstudien in eine Gruppe jüngere Erwachsene $\left(n_{\text {Studien-jung }}=33\right)$ mit einem mittleren Lebensalter von $23.1 \pm 2.7$ (Min. = 19.3, Max. = 34) Jahren und eine Gruppe ältere Erwachsene $\left(n_{\text {Studien-alt }}=9\right)$ mit einem mittleren Lebensalter von $62.7 \pm 12.0$ (Min. $=44.9$, Max. = 83.8) Jahren aufgeteilt.

Die Berechnung von signifikanten Unterschieden wurde mit t-Test und ANOVA durchgeführt. Bei den statistischen Verfahren wurde das Signifikanzniveau mit einem $\alpha$-Niveau von $p \leq .05$ festgesetzt. Um mögliche Verzerrungen der Effektstärkengröße zu vermeiden, wurde die beobachtete Effektstärke durch einen Korrekturfaktor nach Hedges (1981, S. 114) an eine erwartungsgetreue Effektstärke angepasst. Insbesondere bei Studien mit geringem Stichprobenumfang kann es ohne einen Korrekturfaktor zu einer Überschätzung der beobachteten Effektstärke kommen (ebd., S. 112). Die Beurteilung der Effektstärken orientierte sich an Cohen (1992): kleiner Effekt $\geq 0.2$, mittlerer Effekt $\geq 0.5$, großer Effekt $\geq 0.8$. Fehlten entsprechende statistische Angaben zur Berechnung der Effektstärken, so wurden diese nachträglich berechnet. Bei zwei Studien (Sainz de Baranda \& Ayala, 2010; Ylinen et al., 2009) musste die Standardabweichung aus den angegebenen Mittelwerten und Konfidenzintervallen berechnet werden. Bei zwei Studien (Mahieu et al., 2007; Paul, Balakrishnan, \& Izham, 2014) musste die Standardabweichung aus der Stichprobengröße und dem Standardfehler berechnet werden. Zur Berechnung der fehlenden Standardabweichungen wurde die Software Review Manager (Cochrane Collaboration, Version 5.3) verwendet. Für eine Studie (Davis, Ashby, McCale, McQuain, \& Wine, 2005) musste die gepoolte Standardabweichung aufgrund fehlender Streuungsmaße nach Seifert (1991) rekonstruiert werden. In drei Studien (Godges, MacRae, \& Engelke, 1993; Johnson et al., 2014; Muyor, LopezMinarro, \& Casimiro, 2012) mit insgesamt vier Versuchsgruppen wurden die Ergebnisse individuell für das linke und rechte Bein angegeben. Die Berech- 
nung der Effektstärken erfolgte nur für das linke Bein, da sich die Beinseiten hinsichtlich der Effektstärken nicht voneinander unterschieden $(t(3)=1.0$, $p=.4$ ). Die Berechnung der Effektstärken erfolgte immer über den maximalen Untersuchungszeitraum. Wurde beispielsweise eine zehnwöchige Trainingsstudie mit insgesamt vier Messzeitpunkten (t1, t2, t3, t4) durchgeführt, so wurde nur der erste (t1) mit dem letzten Messzeitpunkt (t4) für die Ergebnisdarstellung herangezogen.

\subsection{Ergebnisse}

Es wurden insgesamt 1205 Studien in vier Datenbanken recherchiert. Nachdem die irrelevanten Studien sowie Duplikate im Level 1 Screening entfernt wurden, kamen weitere 26 Studien hinzu, die aus den Literaturverzeichnissen der übriggebliebenen 58 Studien extrahiert wurden (Abb. 19). Nachdem Titel und Abstract (Level 2 Screening) sowie der Volltext (Level 3 Screening) überprüft wurden, blieben zur Auswertung der systematischen Literaturrecherche 42 Primärstudien $\left(N_{\text {Studien }}\right)$ mit insgesamt 1476 Probanden übrig. Es handelt sich insgesamt um eine Hochschulschrift (Schönthaler \& Ott, 1994), ein Buch (Schönthaler \& Ohlendorf, 2002) und 40 Zeitschriftenbeiträge. Drei Zeitschriftenbeiträge (Chagas \& Schmidtbleicher, 2004; Wiemann, 1991; Wydra et al., 1991), die Hochschulschrift und das Buch lagen in deutscher Sprache, alle weiteren Zeitschriftenbeiträge in englischer Sprache vor. Von den 42 Primärstudien verteilen sich $21.4 \%\left(n_{\text {Studien-alt }}=9\right)$ der Studien mit 254 Probanden auf ältere Erwachsene und $78.6 \%\left(n_{\text {Studien-jung }}=33\right)$ der Studien mit 1222 Probanden auf jüngere Erwachsene. Bei drei Studien wurde das Lebensalter der Probanden nur ungenau angegeben. In der Studie von Kerrigan, XenopoulosOddsson, Sullivan, Lelas und Riley (2003) wurde das Lebensalter mit gröBer/gleich 65 Jahren und bei der Studie von Paul et al. (2014) zwischen 18 und 25 Jahren angegeben. Bei Wiemann (1991) wurde kein Lebensalter angegeben. Hier handelte es sich bei den Probanden um Sportstudenten. In keiner Studie wurde ein direkter Vergleich einer Dehnungsintervention zwischen jüngeren und älteren Erwachsenen durchgeführt. 


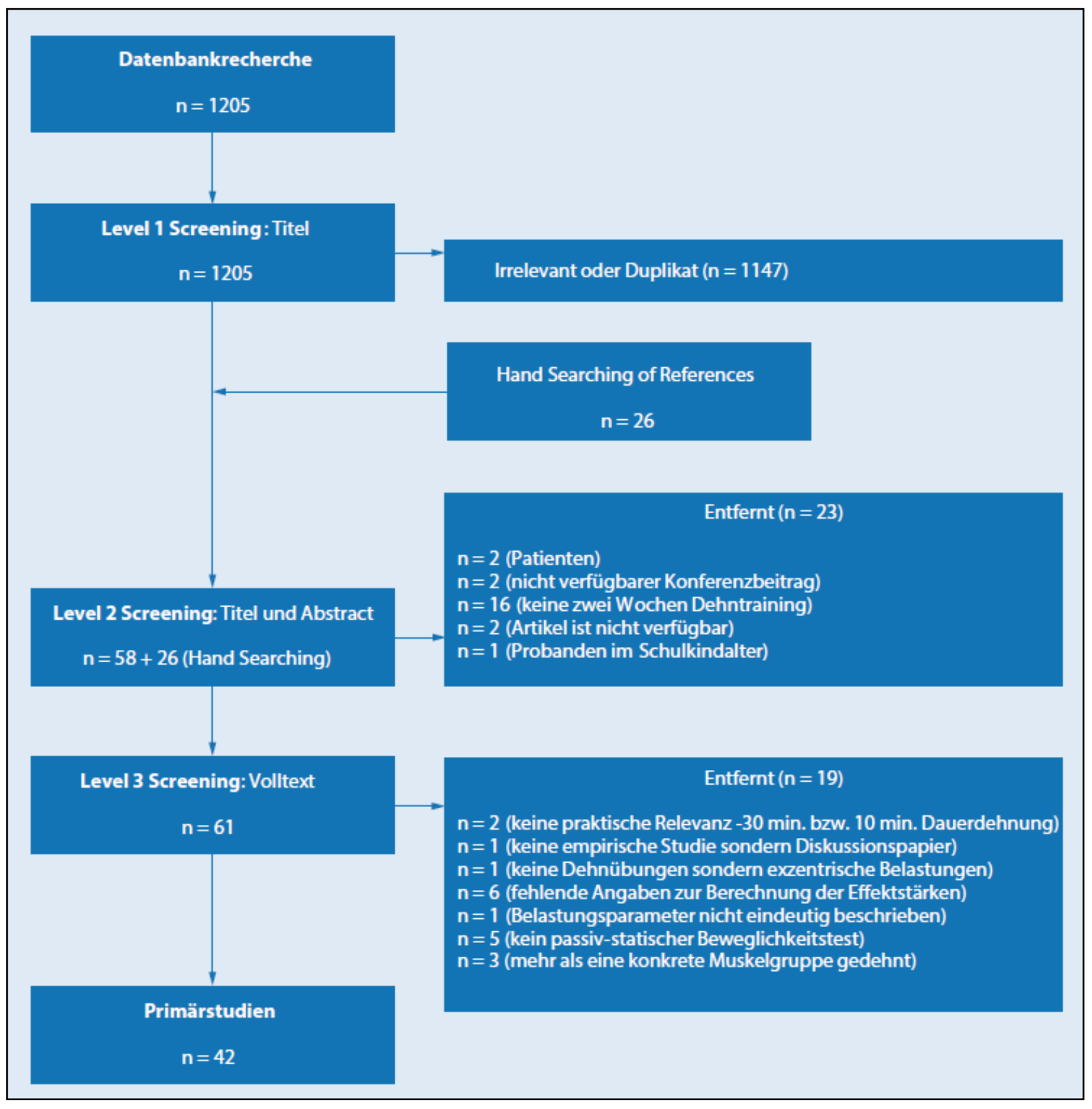

Abb. 19 Fließdiagramm zur Literaturrecherche (Haab, Martini, et al., 2017)

Die Beurteilerübereinstimmung kategorialer Items ergab bei den Items Dehnungsform (Eigen- vs. Fremddehnung), Testgerät (apparativ vs. manuell), gedehnter Muskel (Wadenmuskulatur, Kniestreckmuskulatur, Kniebeugemuskulatur, Hüftbeugemuskulatur) und Dehnintensität (maximal vs. submaximal) laut der Definition von Landis und Koch (1977) eine nahezu vollständige Übereinstimmung der beiden Beurteiler $(k=1.0)$. Ebenfalls wurde eine nahezu vollständige Übereinstimmung bei den Items Dehnmethode (passiv-statisch, pas- 
siv-dynamisch, aktiv-statisch, aktiv-dynamisch, PNF) $(k=0.99)$ sowie der Dehnungsform (Eigen- vs. Fremddehnung) $(k=0.97)$ gefunden. Die Berechnung der Interraterreliabilität bei den kontinuierlichen Items ergab ebenfalls eine nahezu vollständige Übereinstimmung der beiden Beurteiler (Lebensalter, Dauer eines Dehntrainings, Dauer einer Muskeldehnung, Häufigkeit eines Dehntrainings, ROM, Dehnungsspannung: $I C C=1.0$; PEDro-Skala: $I C C=0.94)$. Die mittlere Gesamtreliabilität aller berechneten Koeffizienten zeigt insgesamt eine nahezu vollständige Übereinstimmung der Beurteiler $\left(\kappa_{\text {gesamt }}=0.99\right)$.

In den Primärstudien wurden überwiegend entweder mehrere Dehnmethoden miteinander verglichen oder es fand ein Vergleich zwischen unterschiedlichen Belastungsparametern statt. Somit konnte kein direkter Vergleich der einzelnen Studien hinsichtlich der hier interessierenden unabhängigen Variable „Lebensalter" vorgenommen werden. Aus diesem Grund wurden stattdessen die in den Studien aufgeführten Versuchsgruppen einzeln untersucht. Aus den 42 Primärstudien ( $N_{\text {Studien }}$ ) ergab sich eine Analyse von insgesamt 83 Versuchsgruppen $\left(N_{V G \text {-gesamt }}\right)$, die sich aus 71 Versuchsgruppen mit jüngeren Erwachsenen ( $\left.n_{V G-j u n g}\right)$ und 12 Versuchsgruppen mit älteren Erwachsenen ( $\left.n_{V G-a l t}\right)$ zusammensetzt. Anzumerken ist, dass in der Studie von Wiemann (1991) das Geschlecht separat betrachtet wurde. Aus diesem Grund wurden im Rahmen der metaanalytischen Betrachtung aus der Studie von Wiemann (1991) zwei Versuchsgruppen (eine männliche und eine weibliche Versuchsgruppe) gebildet.

Die mittlere Trainingshäufigkeit in Tagen pro Woche betrug bei den jüngeren Erwachsenen ( $\left.n_{V G-j u n g}=71\right) 4.8 \pm 1.7$ (Min. $=2$, Max. $=7$ ) Tage und die Trainingshäufigkeit über den gesamten Untersuchungszeitraum betrug im Mittel $28.4 \pm 15.5$ (Min. = 6, Max. = 84) Trainingseinheiten. Im Durchschnitt dauerte ein Treatment bei den jüngeren Erwachsenen $6.5 \pm 2.8($ Min. $=2$, Max. $=12)$ Wochen. Die mittlere Trainingshäufigkeit betrug bei den älteren Erwachsenen $\left(n_{V G \text {-alt }}=12\right) 4.6 \pm 2.3$ (Min. $=2$, Max. $\left.=7\right)$ Tage und die Trainingshäufigkeit über den gesamten Untersuchungszeitraum betrug im Mittel $41.3 \pm 46.8$ 
(Min. = 8, Max. = 140) Trainingseinheiten. Im Durchschnitt dauerte ein Treatment bei den älteren Erwachsenen 7.5 $\pm 4.3($ Min. = 2, Max. = 13) Wochen.

Es verteilen sich $85.5 \%$ der Versuchsgruppen auf Dehnungsinterventionen an der Kniebeugemuskulatur ( $\left.n_{V G-j u n g}=63, n_{V G-a / t}=8\right), 3.6 \%$ auf Dehnungsinterventionen der Hüftbeugemuskulatur ( $\left.n_{V G-j u n g}=1, n_{V G-a l t}=2\right), 9.6 \%$ auf Dehnungsinterventionen der Wadenmuskulatur ( $\left.n_{V G-j u n g}=6, n_{V G-a l t}=2\right)$ und $1.2 \%$ auf eine Dehnungsintervention an der Kniestreckmuskulatur $\left(n_{V G-j u n g}=1\right)$.

\subsubsection{Methodologische Qualität der Primärstudien}

Die methodologische Beurteilung der einzelnen Primärstudien befindet sich in Tabelle 5. Die einzelnen Items der modifizierten PEDro-Skala (1 - 14) sind unter der Tabelle beschrieben. Die mittlere methodologische Qualität der 42 Primärstudien beträgt auf der modifizierten PEDro-Skala 7.4. Die Studien mit älteren Erwachsenen $\left(n_{\text {Studien-alt }}=9\right)$ erreichten eine mittlere Bewertung von 6.4 und die Studien mit jüngeren Erwachsenen $\left(n_{\text {Studien-jung }}=33\right)$ erreichten eine mittlere Bewertung von 7.6. Insgesamt wurde bei zwei Studien die Stichprobengröße a priori begründet. Bei 31 Studien wurde ein Kontrollgruppendesign durchgeführt. Bei 33 Studien erfolgte die Aufteilung der Versuchsgruppen randomisiert. In 19 Studien waren die Untersucher, die ein zentrales Outcome gemessen haben, geblindet gegenüber der Intervention der Versuchspersonen. Eine Reliabilität der Outcome-Messungen gaben 29 Studien an. Valide Outcome-Messungen gaben acht Studien an. In keiner Studie wurde berichtet, dass die Zuordnung der Versuchsgruppen verborgen erfolgte (Item 3), dass alle Probanden geblindet waren (Item 5) oder alle Therapeuten geblindet waren (Item 6). 
Tab. 5 Methodologische Beurteilung der Primärstudien mit der modifizierten PEDro-Skala $\left(^{*}=\right.$ Studien mit älteren Erwachsenen) (mod. nach Haab, Martini, et al., 2017)

\begin{tabular}{|c|c|c|c|c|c|c|c|c|c|c|c|c|c|c|c|}
\hline Studie ID & 1 & 2 & 3 & 4 & 5 & 6 & 7 & 8 & 9 & 10 & 11 & 12 & 13 & 14 & $\Sigma$ \\
\hline Ayala (2010) & + & + & - & + & - & - & + & + & + & + & + & - & + & - & 9 \\
\hline Ayala (2013) & + & + & - & - & - & - & + & + & + & + & + & - & + & - & 8 \\
\hline Bandy (1994) & + & + & - & - & - & - & + & + & + & + & + & - & + & - & 8 \\
\hline Bandy (1997) & + & + & - & + & - & - & - & + & + & + & + & - & + & - & 8 \\
\hline Batista $(2009)^{*}$ & + & - & - & - & - & - & - & - & + & + & + & - & - & - & 4 \\
\hline Björklund (2001) & + & + & - & - & - & - & + & + & + & + & + & - & + & + & 9 \\
\hline Chagas (2004) & + & - & - & + & - & - & - & - & + & + & + & - & + & - & 6 \\
\hline Chan (2001) & + & + & - & - & - & - & - & + & + & + & + & - & - & - & 6 \\
\hline Cipriani (2003) & + & + & - & - & - & - & + & - & + & + & + & - & + & - & 7 \\
\hline Cipriani (2012) & + & + & - & + & - & - & + & + & + & + & + & - & + & - & 9 \\
\hline Covert (2010) & + & + & - & + & - & - & + & + & + & + & + & - & + & - & 9 \\
\hline Davis (2005) & + & + & - & + & - & - & + & + & + & + & - & - & + & - & 8 \\
\hline Ferreira (2007) & + & - & - & - & - & - & + & + & + & - & + & - & + & + & 7 \\
\hline Ford (2005) & + & + & - & + & - & - & + & + & + & + & + & - & + & - & 9 \\
\hline Gajdosik (1991) & + & + & - & + & - & - & - & + & + & + & + & - & + & + & 9 \\
\hline Gajdosik (2007) & + & + & - & - & - & - & + & - & + & + & + & - & - & - & 6 \\
\hline Gajdosik $(2005)^{\star}$ & + & + & - & + & - & - & - & + & + & + & + & - & + & - & 8 \\
\hline Gallon $(2011)^{*}$ & + & + & - & - & - & - & - & + & + & + & + & - & - & - & 6 \\
\hline Godges (1993) & + & + & - & - & - & - & - & + & + & + & + & - & + & - & 7 \\
\hline González-Ravé (2012)* & + & - & - & + & - & - & - & + & + & + & + & - & + & - & 7 \\
\hline Gribble (1999) & + & + & - & - & - & - & - & + & + & + & + & - & + & + & 8 \\
\hline Hayes (2012) & + & + & - & - & - & - & - & + & + & + & + & - & + & - & 7 \\
\hline Johnson (2014) & + & + & - & + & - & - & + & - & + & + & + & - & + & - & 8 \\
\hline Johnson $(2007)^{*}$ & + & - & - & - & - & - & - & - & + & - & + & - & + & - & 4 \\
\hline Kerrigan $(2003)^{*}$ & + & + & - & + & - & - & + & + & + & + & + & - & - & - & 8 \\
\hline Konrad (2014) & + & + & - & + & - & - & - & - & + & + & + & - & + & + & 8 \\
\hline La Roche (2006) & + & + & - & + & - & - & - & + & + & + & + & - & - & - & 7 \\
\hline López-Bedoya (2013) & + & + & - & + & - & - & - & + & + & + & + & - & + & - & 8 \\
\hline Mahieu (2009) & + & + & - & + & - & - & + & - & + & + & + & - & + & + & 9 \\
\hline Mahieu (2007) & + & + & - & + & - & - & + & - & + & + & + & - & + & + & 9 \\
\hline Muyor $(2012)^{*}$ & + & + & - & + & - & - & - & + & + & + & + & - & - & - & 7 \\
\hline Paul (2014) & + & + & - & + & - & - & - & + & + & + & + & + & - & - & 8 \\
\hline Rancour (2009) & + & + & - & + & - & - & + & + & + & + & + & - & + & - & 9 \\
\hline Rowlands (2003) & + & + & - & - & - & - & - & + & + & + & + & - & + & - & 7 \\
\hline Sainz de Baranda (2010) & + & + & - & - & - & - & + & - & + & + & + & - & + & - & 7 \\
\hline Schönthaler (1994) & + & - & - & + & - & - & - & + & + & + & + & - & - & - & 6 \\
\hline Schönthaler (2002) & + & + & - & + & - & - & - & + & + & + & + & + & + & - & 9 \\
\hline Watt $(2011)^{\star}$ & + & + & - & - & - & - & + & - & + & + & + & - & - & - & 6 \\
\hline Wiemann (1991) & + & - & - & - & - & - & - & + & + & + & + & - & - & - & 5 \\
\hline Wydra $(1991)^{*}$ & + & - & - & + & - & - & - & + & + & + & + & - & + & + & 8 \\
\hline
\end{tabular}


Tab. 5 (Fortsetzung)

\begin{tabular}{|l|c|c|c|c|c|c|c|c|c|c|c|c|c|c|c|}
\hline Ylinen (2009) & + & - & - & + & - & - & - & - & + & + & + & - & - & - & 5 \\
\hline Yuktasir (2009) & + & + & - & - & - & - & + & + & + & + & + & - & - & - & 7 \\
\hline
\end{tabular}

1. Die Ein- und Ausschlusskriterien wurden spezifiziert.

2. Die Probanden wurden den Gruppen randomisiert zugeordnet.

3. Die Zuordnung der Gruppen erfolgte verborgen.

4. Zu Beginn der Studie waren die Gruppen bzgl. der wichtigsten prognostischen Indikatoren einander ähnlich.

5. Alle Probanden waren geblindet.

6. Alle Therapeuten/innen, die eine Therapie durchgeführt haben, waren geblindet.

7. Alle Untersucher, die zumindest ein zentrales Outcome gemessen haben, waren geblindet.

8. Von mehr als $85 \%$ der ursprünglich den Gruppen zugeordneten Probanden wurde ein zentrales Outcome gemessen.

9. Alle Probanden, die für die Ergebnismessungen zur Verfügung standen, haben die Behandlung oder Kontrollanwendung bekommen wie zugeordnet.

10. Für mindestens ein zentrales Outcome wurden die Ergebnisse statistischer Gruppenvergleiche berichtet.

11. Die Studie berichtet sowohl Punkt- als auch Streuungsmaße für zumindest ein zentrales Outcome.

12. Die Stichprobengröße wurde a priori begründet.

13. Die Outcome-Messungen waren reliabel.

14. Die Outcome-Messungen waren valide.

\subsubsection{Darstellung der Primärstudien}

Nachfolgende Tabellen stellen die Studiendetails der im systematischen Review erhobenen Primärstudien dar. Die Tabellen sind in der Reihenfolge nach dem Erstautor der Primärstudien sortiert. Zur besseren Lesbarkeit wurden nur die Namen der Erstautoren und das Publikationsjahr aufgeführt. Des Weiteren unterteilen sich die Tabellen in Studien zur Erhebung der maximalen Beweglichkeit an der Kniebeugemuskulatur (Tab. 6), der Kniestreckmuskulatur (Tab. 7), der Hüftbeugemuskulatur (Tab. 8), der Wadenmuskulatur (Tab. 9) sowie der maximalen Dehnungsspannung an der Kniebeugemuskulatur (Tab. 10) und der Wadenmuskulatur (Tab. 11). 


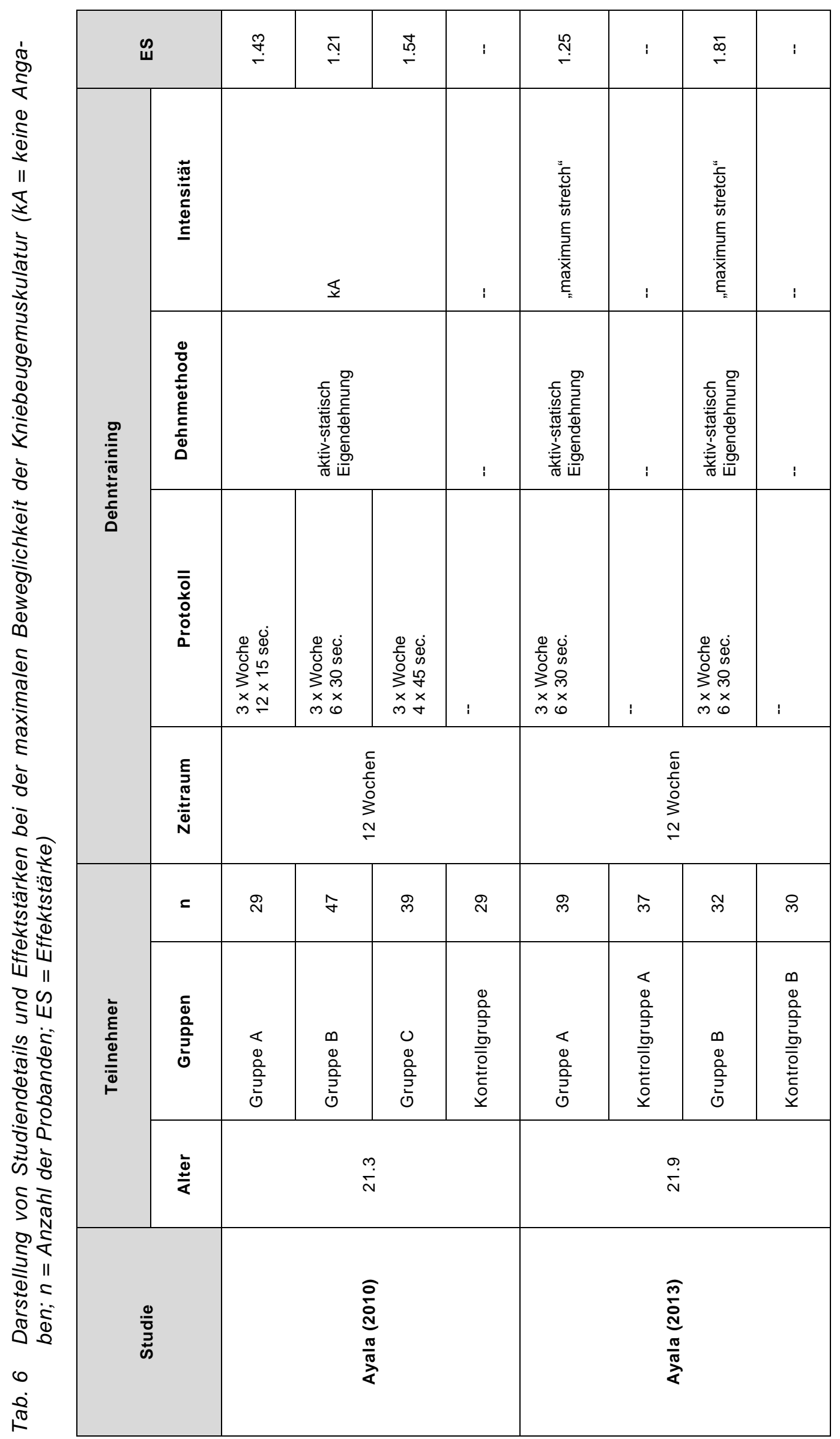




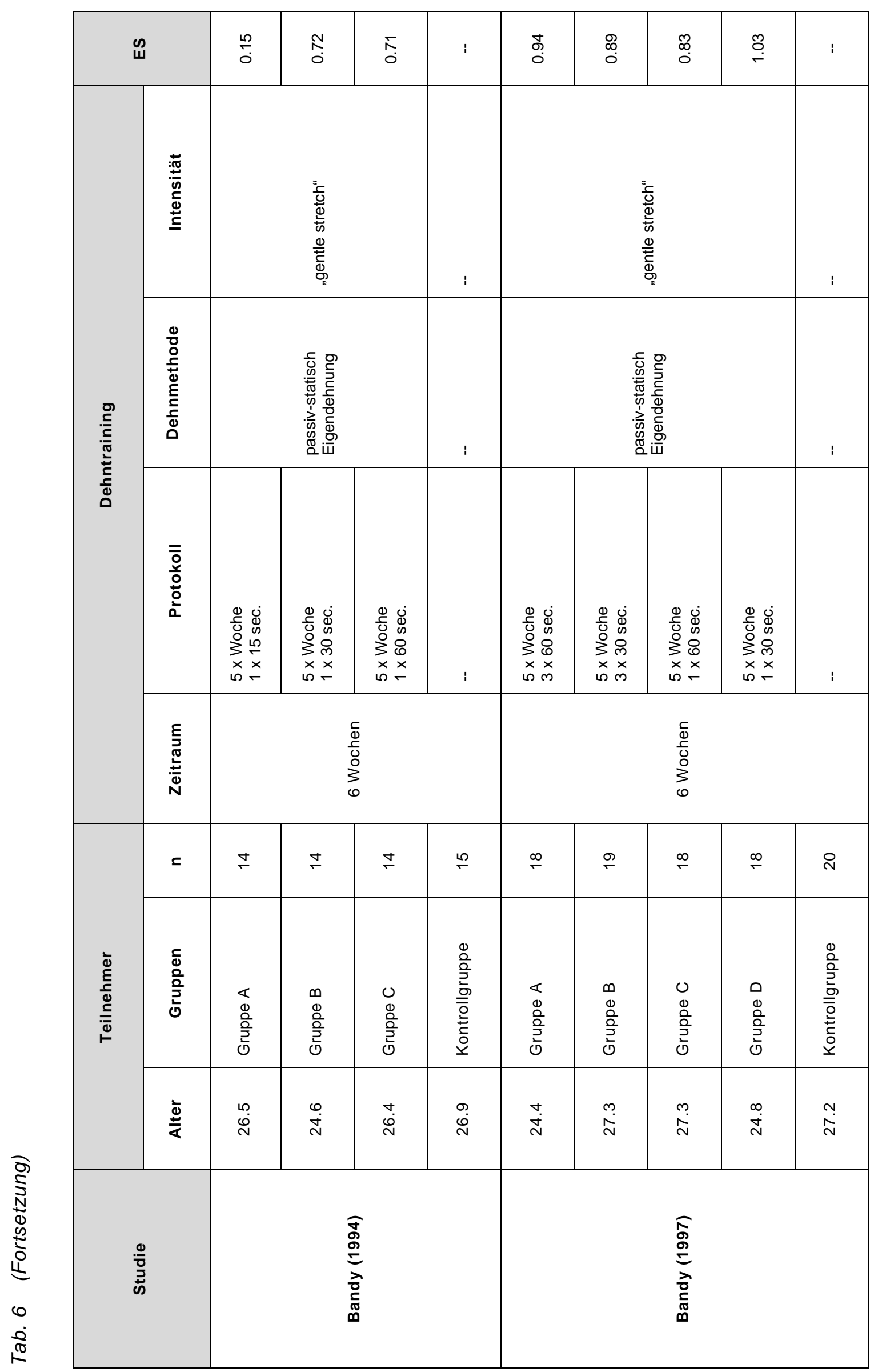




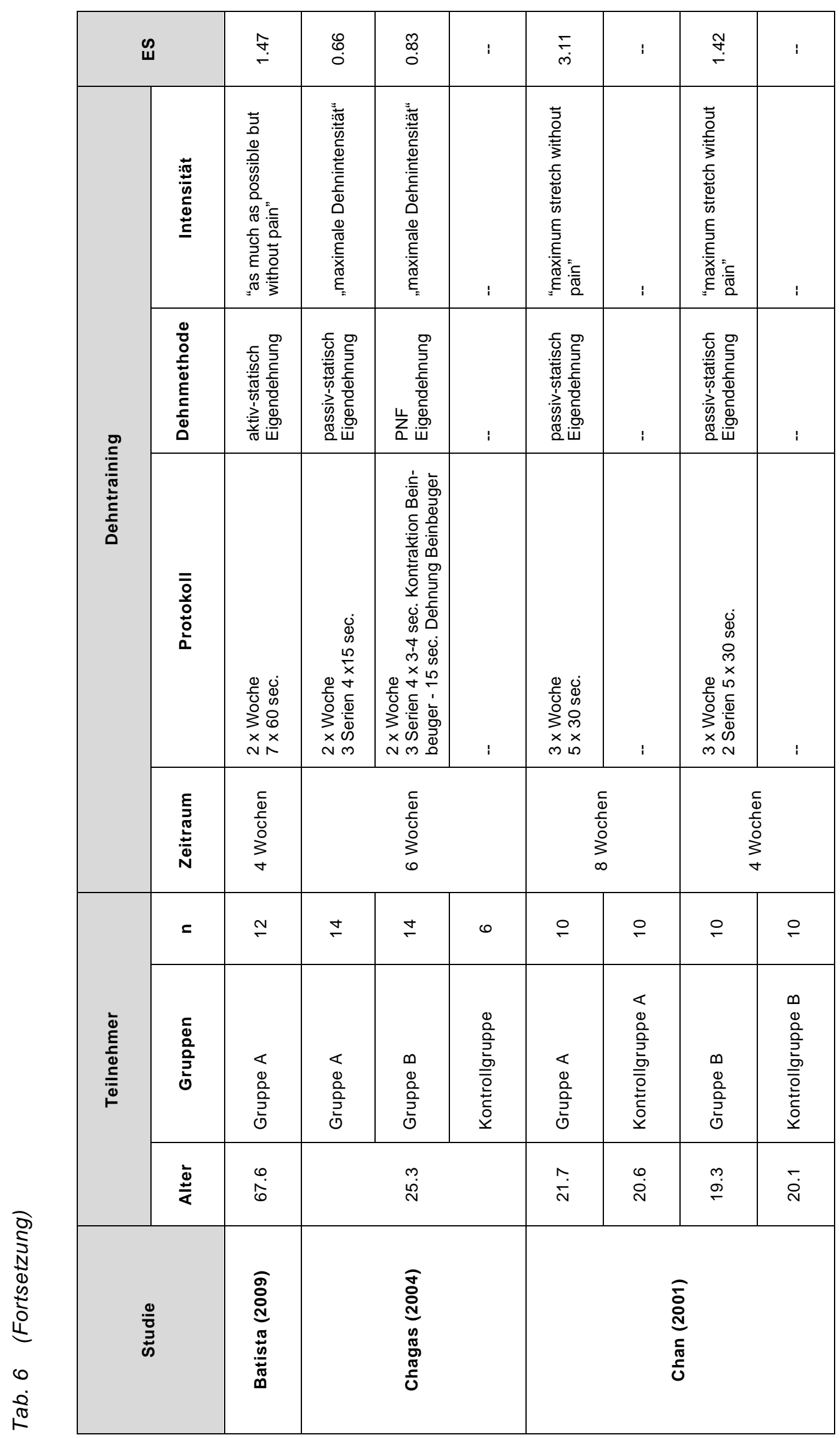


Altersspezifische Anpassungen an ein Dehntraining: Ein systematischer Review

63

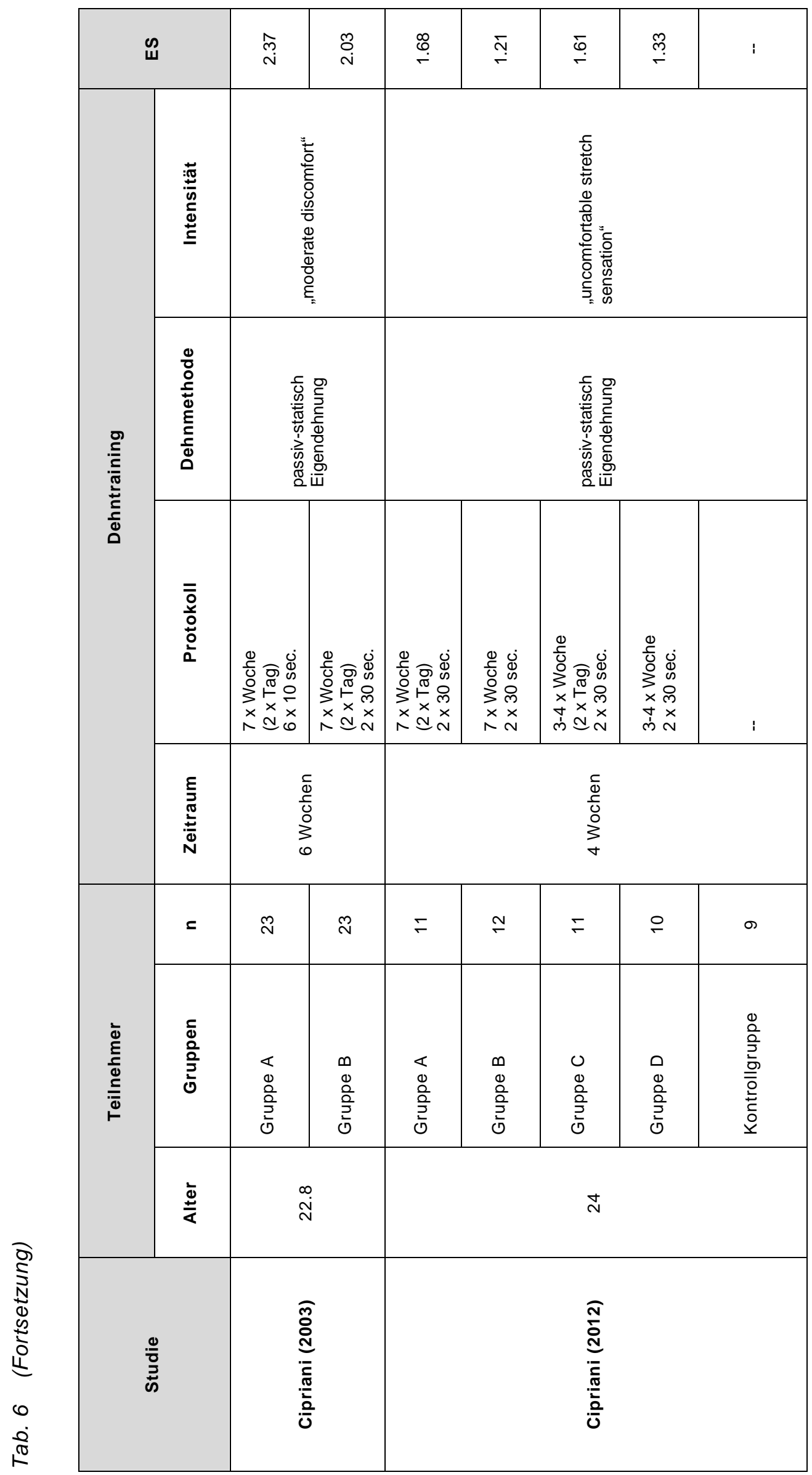




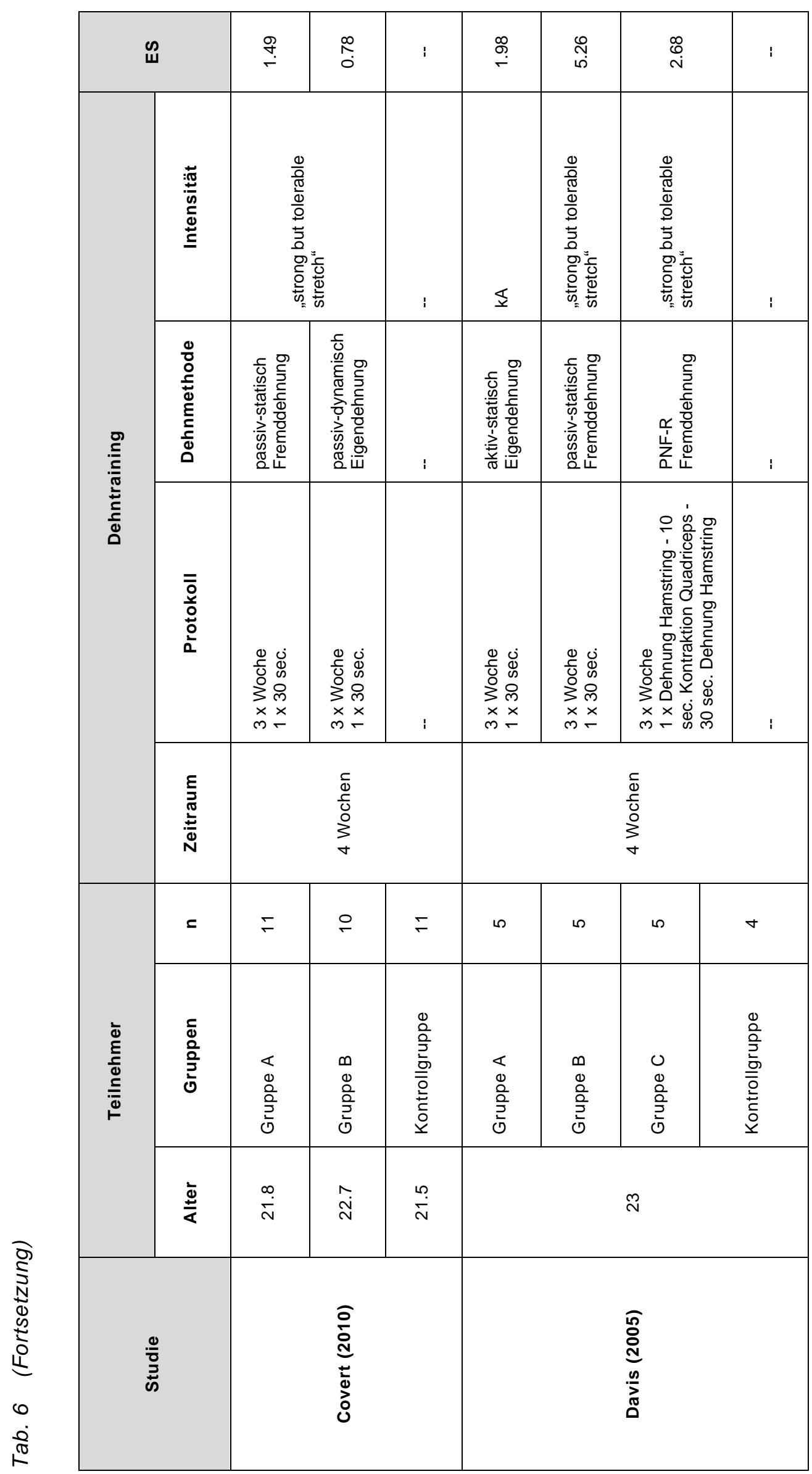




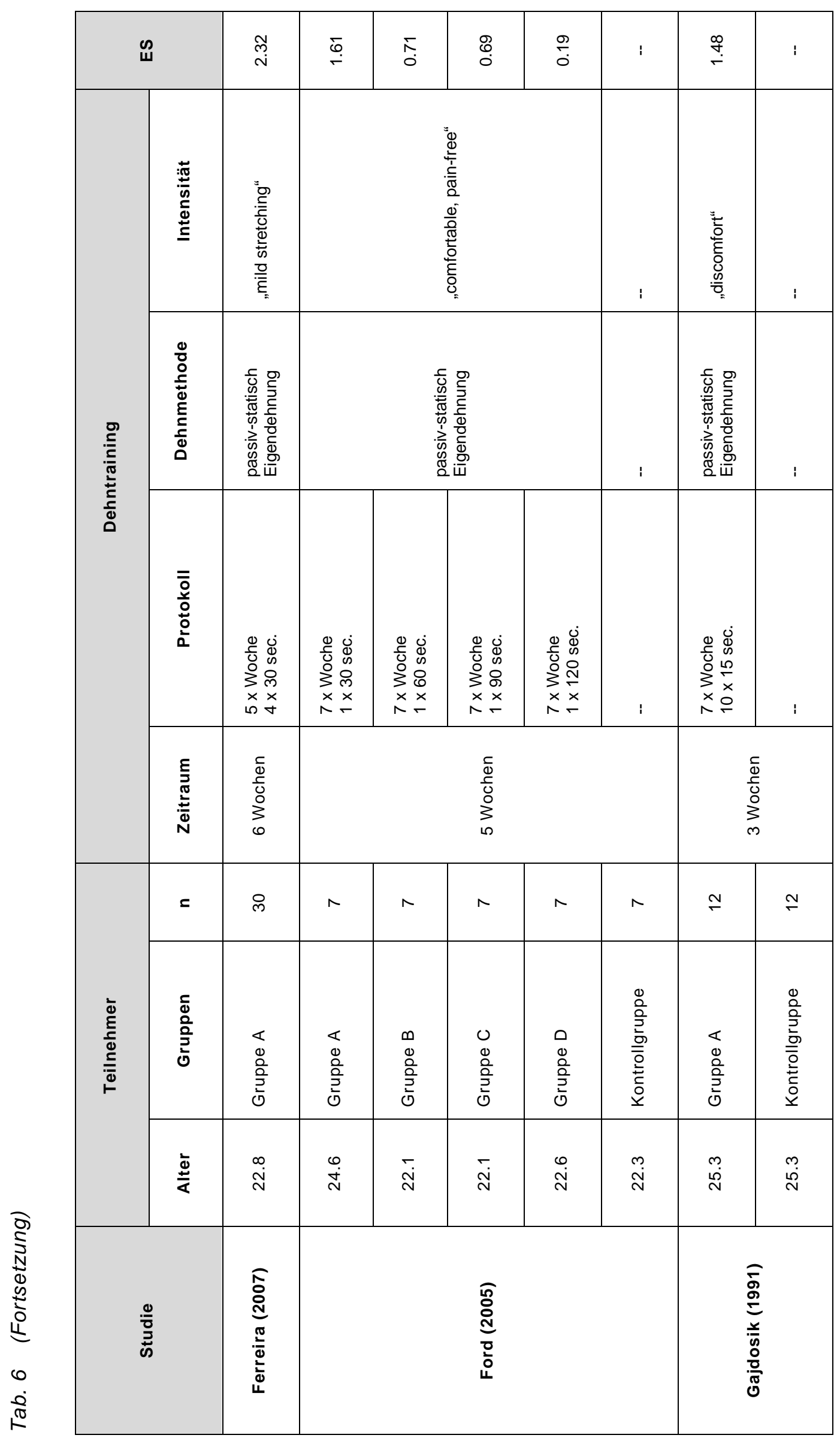




\begin{tabular}{|c|c|c|c|c|c|c|c|c|c|c|}
\hline \multicolumn{2}{|c|}{ 邑 } & $\begin{array}{l}\text { ஜ } \\
0\end{array}$ & ; & $\stackrel{\sigma}{\check{r}}$ & 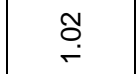 & : & $\stackrel{\text { @্ }}{r}$ & 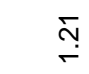 & & : \\
\hline \multirow{4}{*}{ 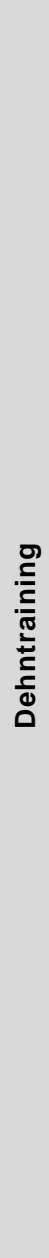 } & 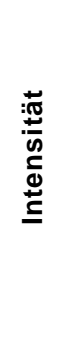 & 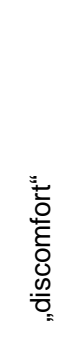 & : & 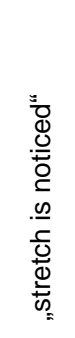 & $\mathbb{\Sigma}$ & : & \multicolumn{3}{|c|}{ 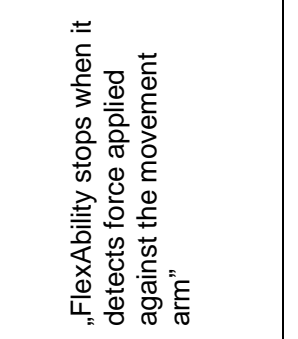 } & i \\
\hline & 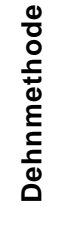 & 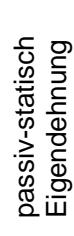 & ; & 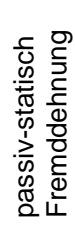 & 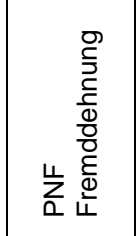 & ; & 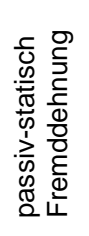 & \multicolumn{2}{|c|}{ 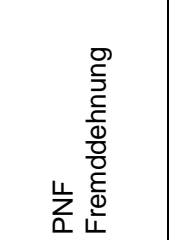 } & ; \\
\hline & $\begin{array}{l}\overline{\overline{0}} \\
\text { o } \\
\dot{0} \\
\vdots \\
0\end{array}$ & 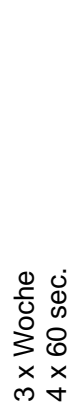 & : & 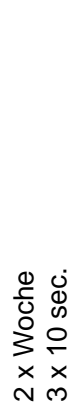 & 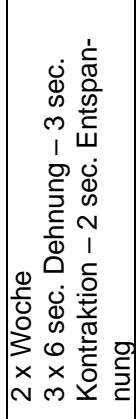 & : & 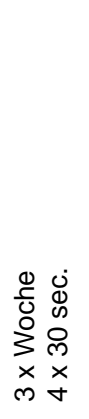 & \multicolumn{2}{|c|}{ 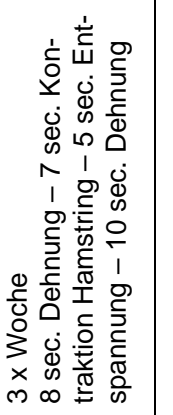 } & : \\
\hline & 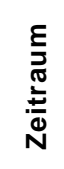 & \multicolumn{2}{|c|}{$\infty$} & \multicolumn{3}{|c|}{$\begin{array}{l}\frac{c}{0} \\
\frac{1}{0} \\
0 \\
3 \\
m \\
\end{array}$} & \multicolumn{4}{|c|}{\begin{tabular}{l}
\multicolumn{1}{c}{} \\
$\Phi$ \\
$\frac{1}{0}$ \\
0 \\
0 \\
0
\end{tabular}} \\
\hline \multirow{3}{*}{ 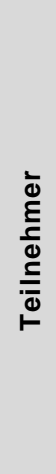 } & $=$ & $\infty$ & $\sigma$ & 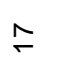 & 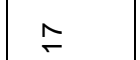 & $\stackrel{\varphi}{\circ}$ & $\cong$ & $\underset{\tau}{ }$ & $\stackrel{0}{\underline{0}}$ & \\
\hline & $\begin{array}{l}\frac{5}{0} \\
\frac{0}{0} \\
\frac{2}{2} \\
0\end{array}$ & $\begin{array}{l}\varangle \\
0 \\
\frac{0}{2} \\
\frac{2}{2} \\
0\end{array}$ & 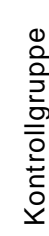 & $\begin{array}{l}\varangle \\
0 \\
\frac{0}{2} \\
\frac{2}{2} \\
0\end{array}$ & $\begin{array}{l}\infty \\
0 \\
0 \\
00 \\
0 \\
0 \\
0\end{array}$ & 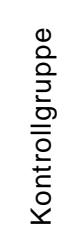 & $\begin{array}{l}\varangle \\
0 \\
0 \\
00 \\
2 \\
0 \\
0\end{array}$ & $\begin{array}{l}\infty \\
0 \\
00 \\
\frac{0}{2} \\
\frac{2}{0}\end{array}$ & & \\
\hline & 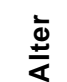 & \multicolumn{2}{|c|}{$\hat{\varphi}$} & $\begin{array}{l}n \\
\ddot{\theta} \\
0\end{array}$ & $\stackrel{\sim}{\dot{0}}$ & $\stackrel{\forall}{\dot{0}}$ & \multicolumn{4}{|c|}{$\hat{\sim}$} \\
\hline & & \multicolumn{2}{|c|}{ 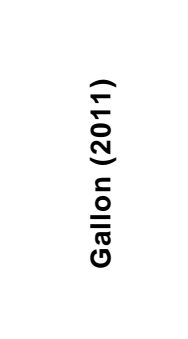 } & \multicolumn{3}{|c|}{ 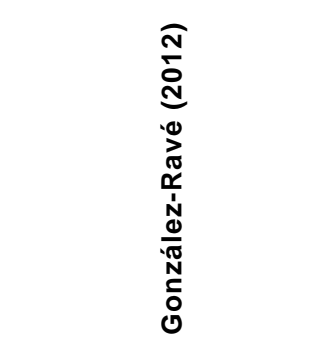 } & \multicolumn{3}{|c|}{ 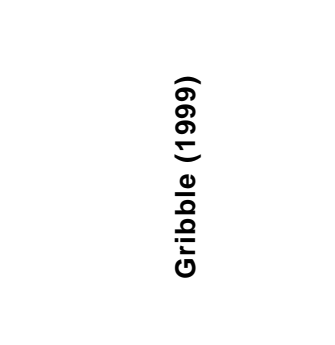 } & \\
\hline
\end{tabular}




\begin{tabular}{|c|c|c|c|c|c|c|c|c|c|c|}
\hline \multicolumn{2}{|c|}{ 邑 } & $\stackrel{\stackrel{\leftrightarrow}{\leftrightarrow}}{\stackrel{\leftrightarrow}{-}}$ & $\stackrel{\text { ల్}}{r}$ & : & $\stackrel{\text { L̊ }}{\circ}$ & \&: & : & $\begin{array}{l}\text { g. } \\
\text { O }\end{array}$ & $\stackrel{\llcorner}{\rightleftarrows}$ & $\stackrel{ }{\stackrel{ }{r}}$ \\
\hline \multirow{4}{*}{ 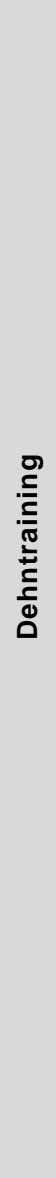 } & 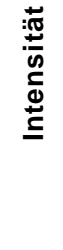 & 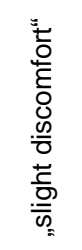 & 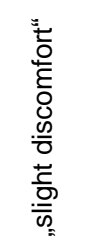 & : & \multicolumn{2}{|c|}{ 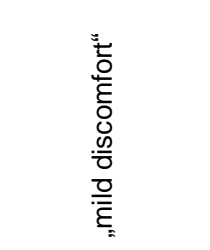 } & : & $\mathbb{\Sigma}$ & 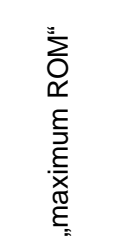 & 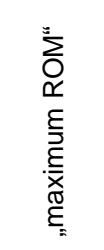 \\
\hline & 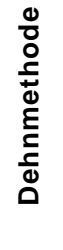 & 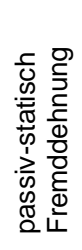 & 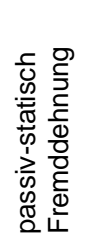 & $i$ & 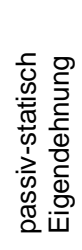 & 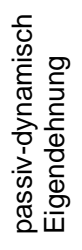 & : & 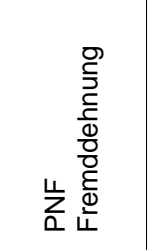 & 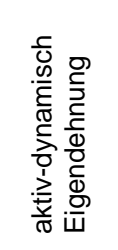 & 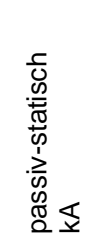 \\
\hline & $\begin{array}{l}\overline{\overline{0}} \\
\text { 응 } \\
\frac{0}{0} \\
\frac{0}{0}\end{array}$ & 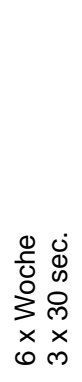 & 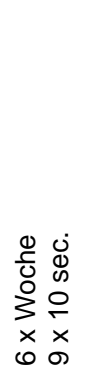 & : & 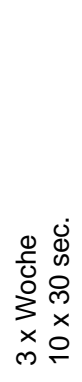 & 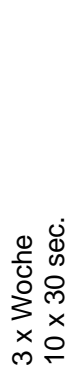 & : & 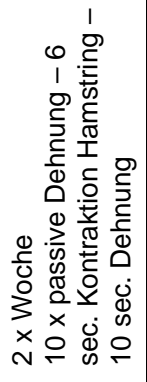 & 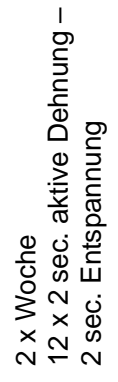 & 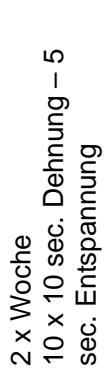 \\
\hline & 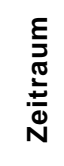 & \multicolumn{3}{|c|}{$\begin{array}{l}\frac{c}{0} \\
\frac{0}{0} \\
\vdots \\
0 \\
0\end{array}$} & \multicolumn{3}{|c|}{$\begin{array}{l}\frac{c}{\Phi} \\
\frac{5}{0} \\
0 \\
3 \\
\forall\end{array}$} & \multicolumn{3}{|c|}{$\begin{array}{l}\frac{5}{0} \\
\frac{1}{0} \\
0 \\
3 \\
0\end{array}$} \\
\hline \multirow{3}{*}{ 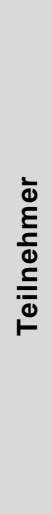 } & $=$ & $\cong$ & $\stackrel{\nabla}{\sim}$ & $\infty$ & $\sigma$ & 으 & 우 & $\sigma$ & $\infty$ & $\mp$ \\
\hline & $\begin{array}{l}\frac{1}{0} \\
\frac{0}{2} \\
\frac{2}{2} \\
0\end{array}$ & $\begin{array}{l}\varangle \\
0 \\
00 \\
\frac{0}{2} \\
\frac{0}{1}\end{array}$ & 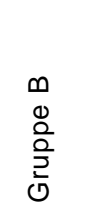 & 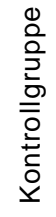 & 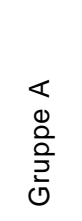 & 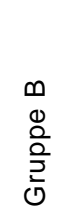 & 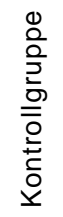 & $\begin{array}{l}\varangle \\
\Phi \\
\frac{0}{2} \\
2 \\
\frac{0}{0}\end{array}$ & $\begin{array}{l}\infty \\
0 \\
0 \\
\frac{0}{2} \\
\frac{2}{0} \\
0\end{array}$ & $\begin{array}{l}0 \\
0 \\
\frac{0}{2} \\
\frac{2}{2} \\
0\end{array}$ \\
\hline & $\stackrel{\ddagger}{\stackrel{亠}{\varangle}}$ & \multicolumn{3}{|c|}{$\tilde{N}$} & $\bar{m}$ & $\stackrel{\substack{\infty \\
\sim}}{\sim}$ & 庐 & \multicolumn{3}{|c|}{$\stackrel{\infty}{\stackrel{\infty}{N}}$} \\
\hline & & \multicolumn{3}{|c|}{ 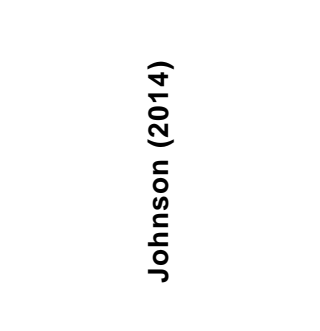 } & \multicolumn{3}{|c|}{ 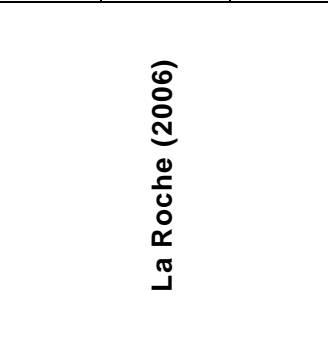 } & \multicolumn{3}{|c|}{ 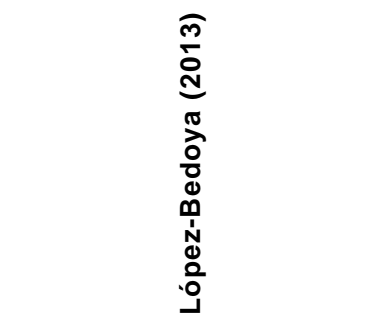 } \\
\hline
\end{tabular}




\begin{tabular}{|c|c|c|c|c|c|c|c|c|c|}
\hline \multicolumn{2}{|c|}{ 号 } & \multirow{2}{*}{ 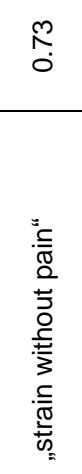 } & \multirow[t]{2}{*}{ : } & \multirow{2}{*}{ 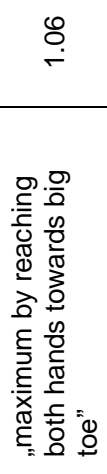 } & \multirow{2}{*}{ 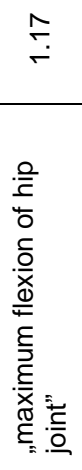 } & \multirow{2}{*}{ 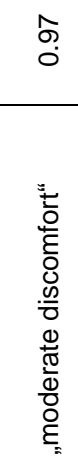 } & \multirow{2}{*}{$\begin{array}{l}\text { ' } \\
\\
\end{array}$} & \multicolumn{2}{|c|}{ بم } \\
\hline \multirow{5}{*}{ 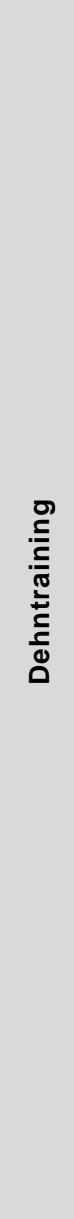 } & 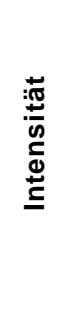 & & & & & & & & \\
\hline & 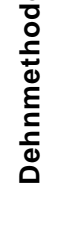 & 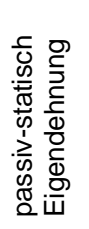 & : & 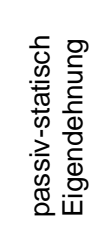 & 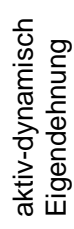 & 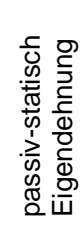 & : & & \\
\hline & $\begin{array}{l}\overline{\bar{o}} \\
\frac{8}{0} \\
\frac{0}{0} \\
\dot{0}\end{array}$ & 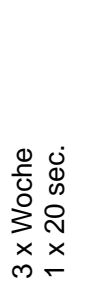 & : & 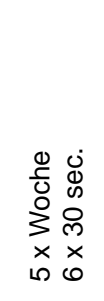 & $\begin{array}{ll}0 & \dot{0} \\
\frac{5}{0} & \mathbb{0} \\
0 & 0 \\
3 & 0 \\
\times & \times \\
1 & 0\end{array}$ & 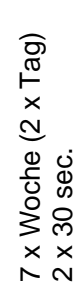 & : & 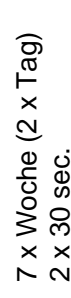 & 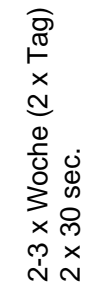 \\
\hline & & \multirow{2}{*}{\multicolumn{2}{|c|}{$\begin{array}{l}\frac{1}{0} \\
\frac{1}{0} \\
0 \\
3 \\
\stackrel{N}{N}\end{array}$}} & \multirow{2}{*}{\multicolumn{2}{|c|}{ 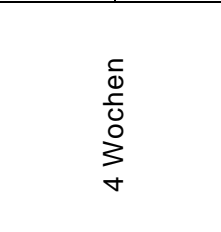 }} & $\nabla$ & $\nabla$ & $\nabla$ & $\sigma$ \\
\hline & 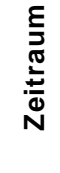 & & & & & & & & \\
\hline \multirow{3}{*}{ 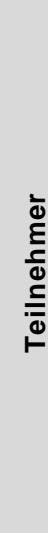 } & $=$ & $\hat{N}$ & হ & $\hat{m}$ & $\hat{m}$ & & & & \\
\hline & 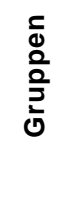 & $\begin{array}{l}\varangle \\
\searrow \\
\frac{0}{2} \\
\frac{2}{2} \\
0\end{array}$ & 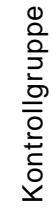 & $\begin{array}{l}\varangle \\
\searrow \\
\frac{0}{2} \\
\frac{0}{2} \\
0\end{array}$ & $\begin{array}{l}\infty \\
0 \\
\stackrel{0}{0} \\
\frac{0}{3} \\
\overline{0}\end{array}$ & & & & \\
\hline & 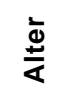 & $\stackrel{\leftrightarrow}{\stackrel{+}{\forall}}$ & 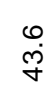 & & & & & & \\
\hline & & 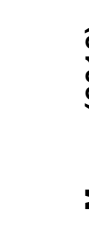 & & & & & & & \\
\hline
\end{tabular}




\begin{tabular}{|c|c|c|c|c|}
\hline \multicolumn{2}{|c|}{ 邑 } & 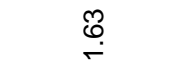 & 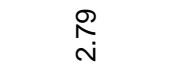 & : \\
\hline \multirow{4}{*}{ 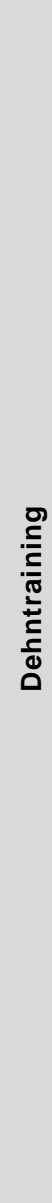 } & 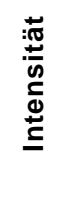 & 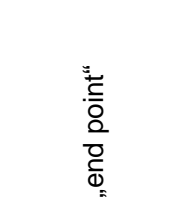 & 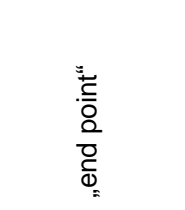 & : \\
\hline & 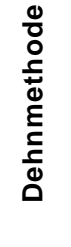 & 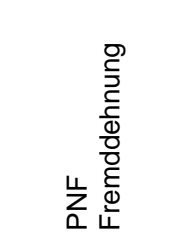 & 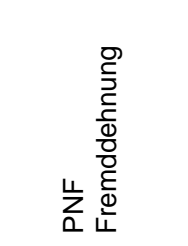 & : \\
\hline & $\begin{array}{l}\overline{\overline{0}} \\
\text { 응 } \\
\frac{0}{0} \\
\frac{0}{0}\end{array}$ & 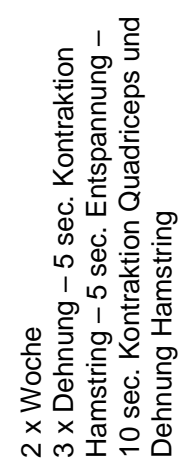 & 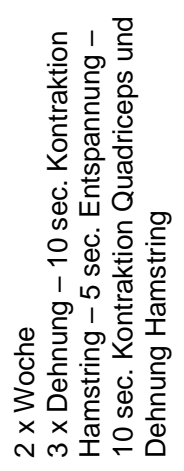 & : \\
\hline & 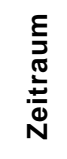 & \multicolumn{3}{|c|}{$\begin{array}{l}\frac{1}{0} \\
\frac{1}{0} \\
0 \\
\vdots \\
0\end{array}$} \\
\hline \multirow{3}{*}{ 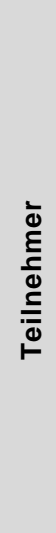 } & c & $\mp$ & $\stackrel{m}{\sim}$ & $\stackrel{m}{\longrightarrow}$ \\
\hline & $\begin{array}{l}\frac{}{0} \\
\frac{0}{2} \\
\frac{2}{2} \\
0\end{array}$ & $\begin{array}{l}\varangle \\
0 \\
\frac{0}{0} \\
\sum_{0} \\
0\end{array}$ & $\begin{array}{l}\infty \\
0 \\
\frac{0}{2} \\
\frac{2}{3} \\
0\end{array}$ & 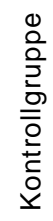 \\
\hline & 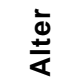 & \multicolumn{3}{|c|}{ 尺 } \\
\hline & & \multicolumn{3}{|c|}{ 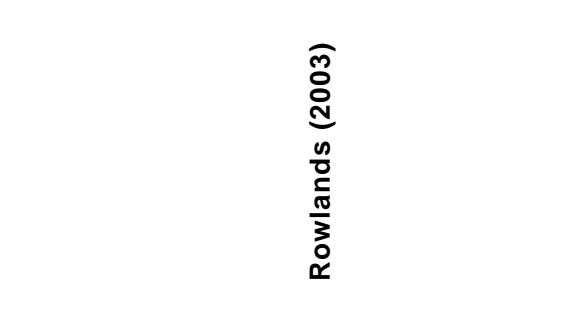 } \\
\hline
\end{tabular}




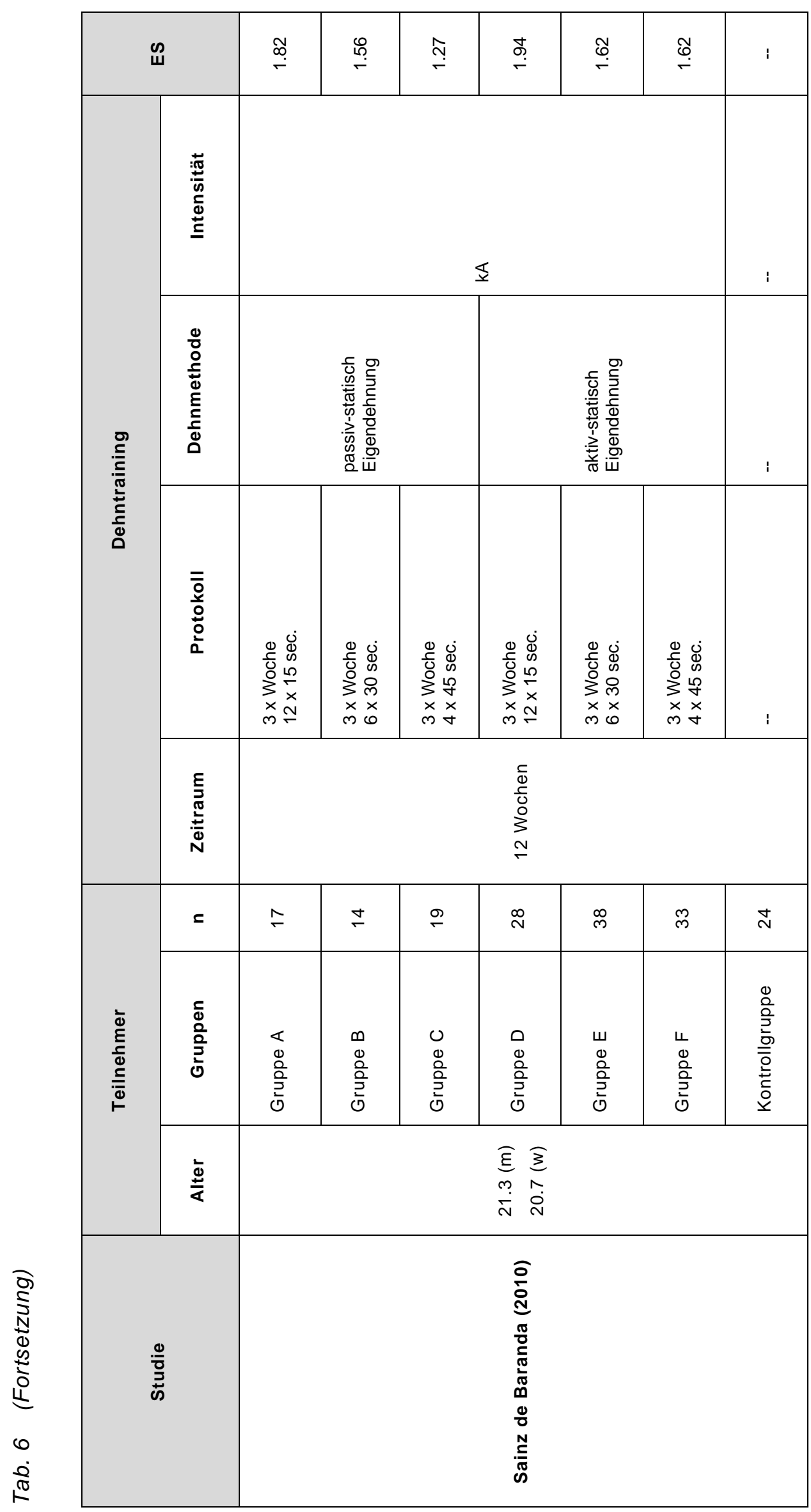




\begin{tabular}{|c|c|c|c|c|c|c|c|c|c|}
\hline \multicolumn{2}{|c|}{ 邑 } & 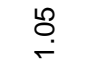 & $\stackrel{\infty}{\stackrel{\infty}{\circ}}$ & 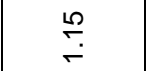 & ; & $\begin{array}{l}\infty \\
\stackrel{\infty}{0} \\
\stackrel{0}{0}\end{array}$ & : & \begin{tabular}{l}
\multirow{0}{0}{} \\
$\stackrel{0}{0}$
\end{tabular} & 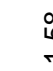 \\
\hline \multirow{4}{*}{ 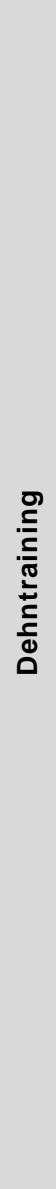 } & 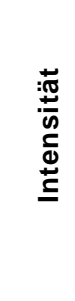 & \multicolumn{3}{|c|}{ 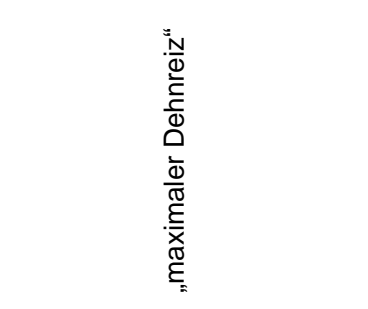 } & : & 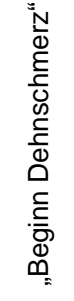 & : & \multicolumn{2}{|c|}{ 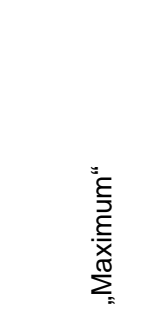 } \\
\hline & 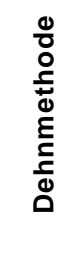 & 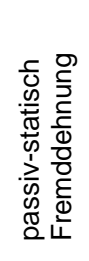 & 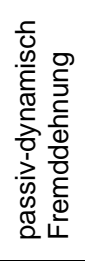 & 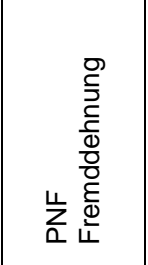 & : & 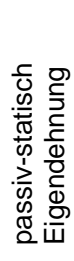 & : & \multicolumn{2}{|c|}{ 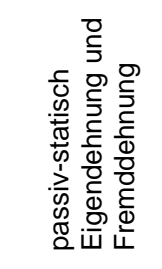 } \\
\hline & $\begin{array}{l}\overline{\bar{o}} \\
\frac{8}{o} \\
\overline{0} \\
\bar{a}\end{array}$ & 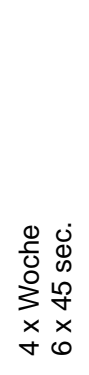 & 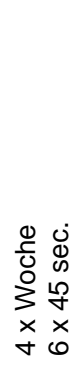 & 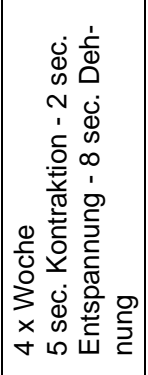 & i & 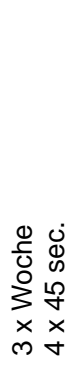 & : & \multicolumn{2}{|c|}{ 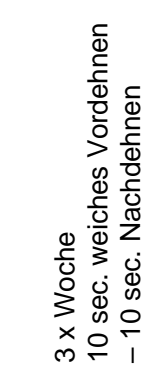 } \\
\hline & 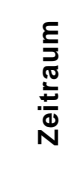 & \multicolumn{4}{|c|}{$\begin{array}{l}\frac{D}{\Phi} \\
\frac{d}{U} \\
0 \\
3 \\
\forall\end{array}$} & \multicolumn{2}{|c|}{ 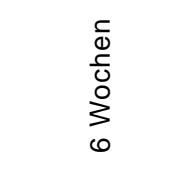 } & \multicolumn{2}{|c|}{ 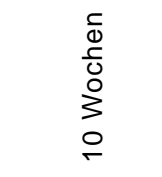 } \\
\hline \multirow{3}{*}{ 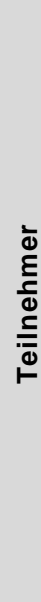 } & c & $\sigma$ & $\wedge$ & $\mp$ & $\sigma$ & $\simeq$ & $\simeq$ & $\stackrel{\llcorner}{\sim}$ & $\stackrel{L}{a}$ \\
\hline & $\begin{array}{l}\Xi \\
\vdots \\
\frac{0}{2} \\
\frac{2}{2} \\
0\end{array}$ & $\begin{array}{l}\varangle \\
0 \\
00 \\
\frac{0}{2} \\
0 \\
0\end{array}$ & $\begin{array}{l}\infty \\
\mathbb{0} \\
\frac{0}{2} \\
\frac{2}{2} \\
0\end{array}$ & 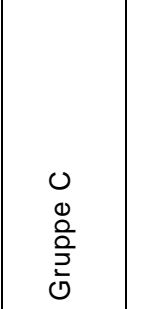 & 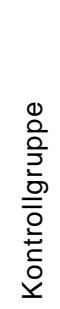 & $\begin{array}{l}\nwarrow \\
\searrow \\
\frac{0}{2} \\
\frac{2}{2} \\
0\end{array}$ & 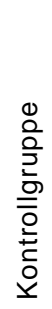 & 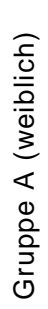 & 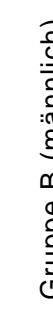 \\
\hline & 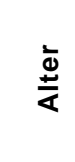 & \multicolumn{4}{|c|}{ 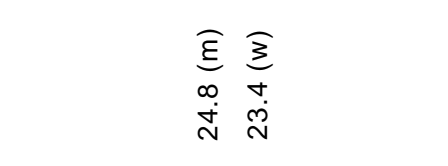 } & 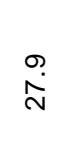 & $\stackrel{m}{\stackrel{m}{N}}$ & \multicolumn{2}{|c|}{ 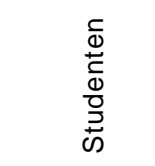 } \\
\hline & & \multicolumn{4}{|c|}{ 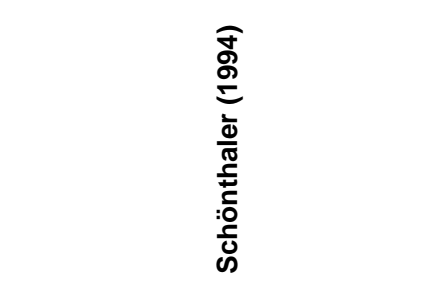 } & \multicolumn{2}{|c|}{ 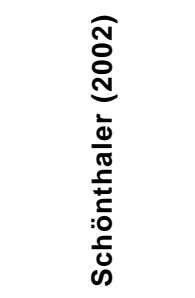 } & \multicolumn{2}{|c|}{ 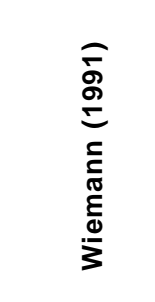 } \\
\hline
\end{tabular}




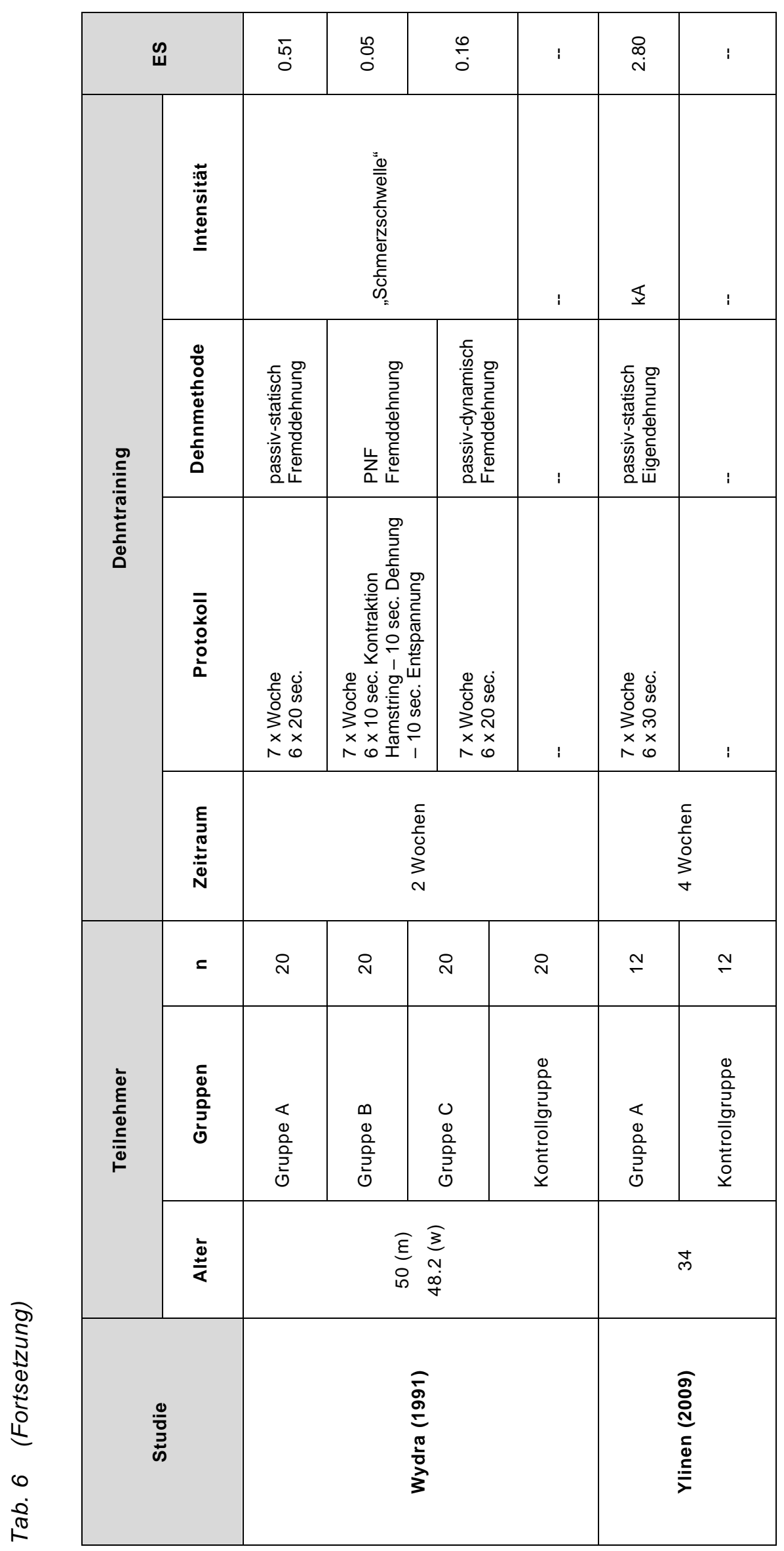




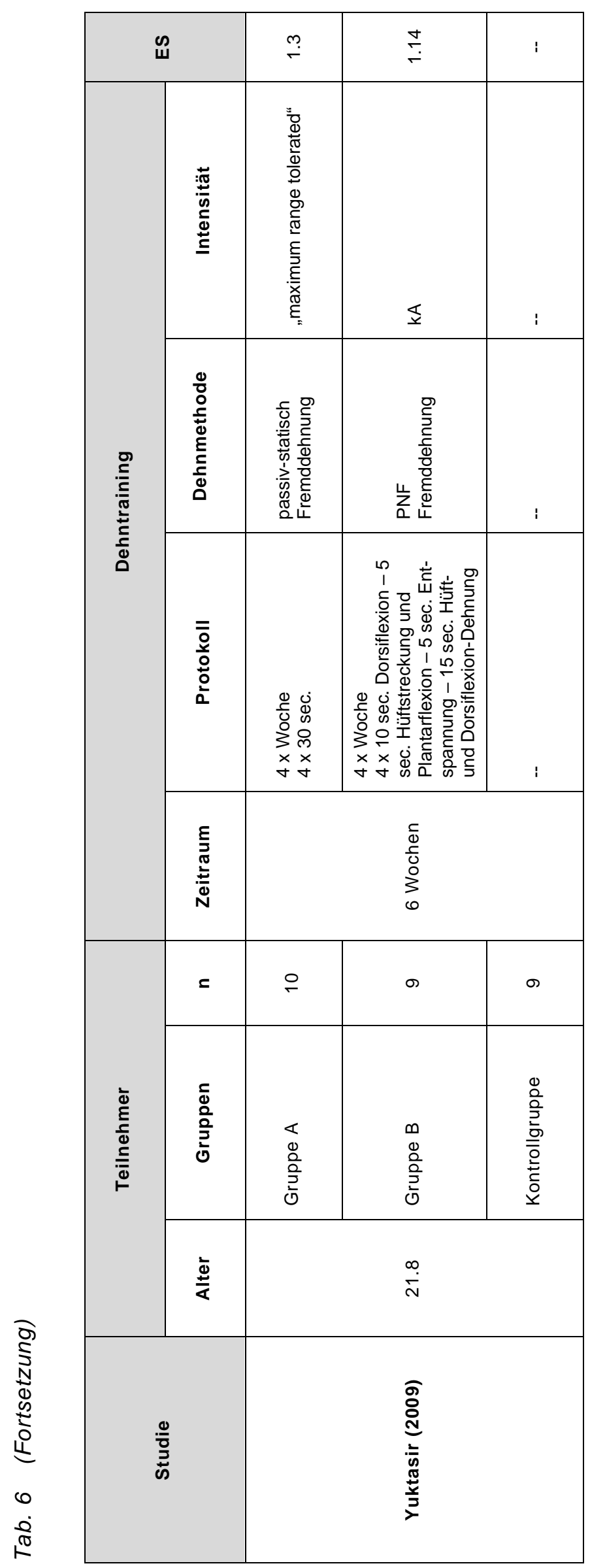




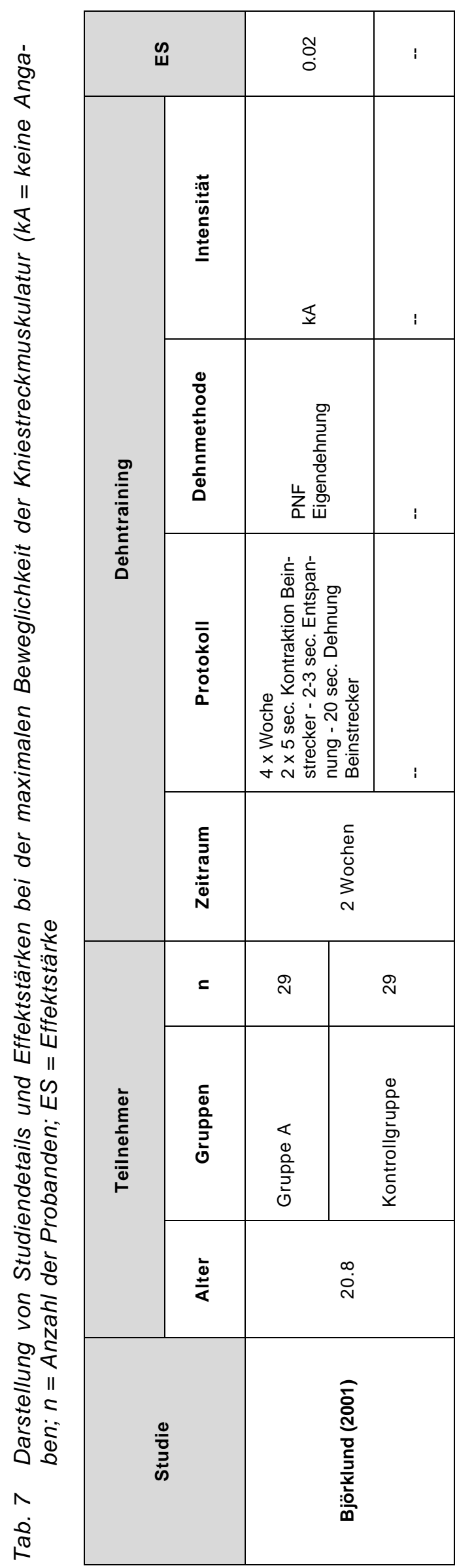




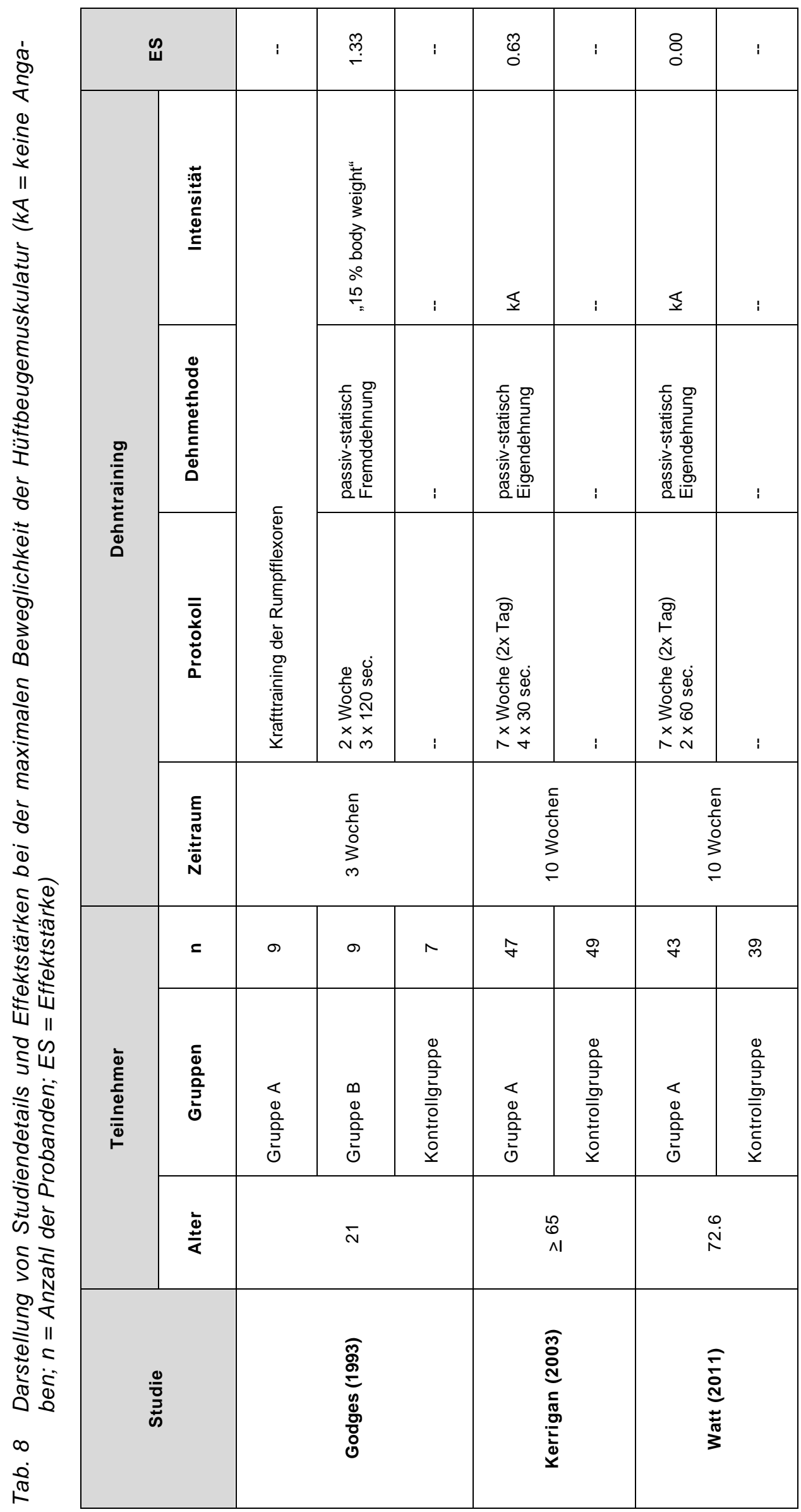




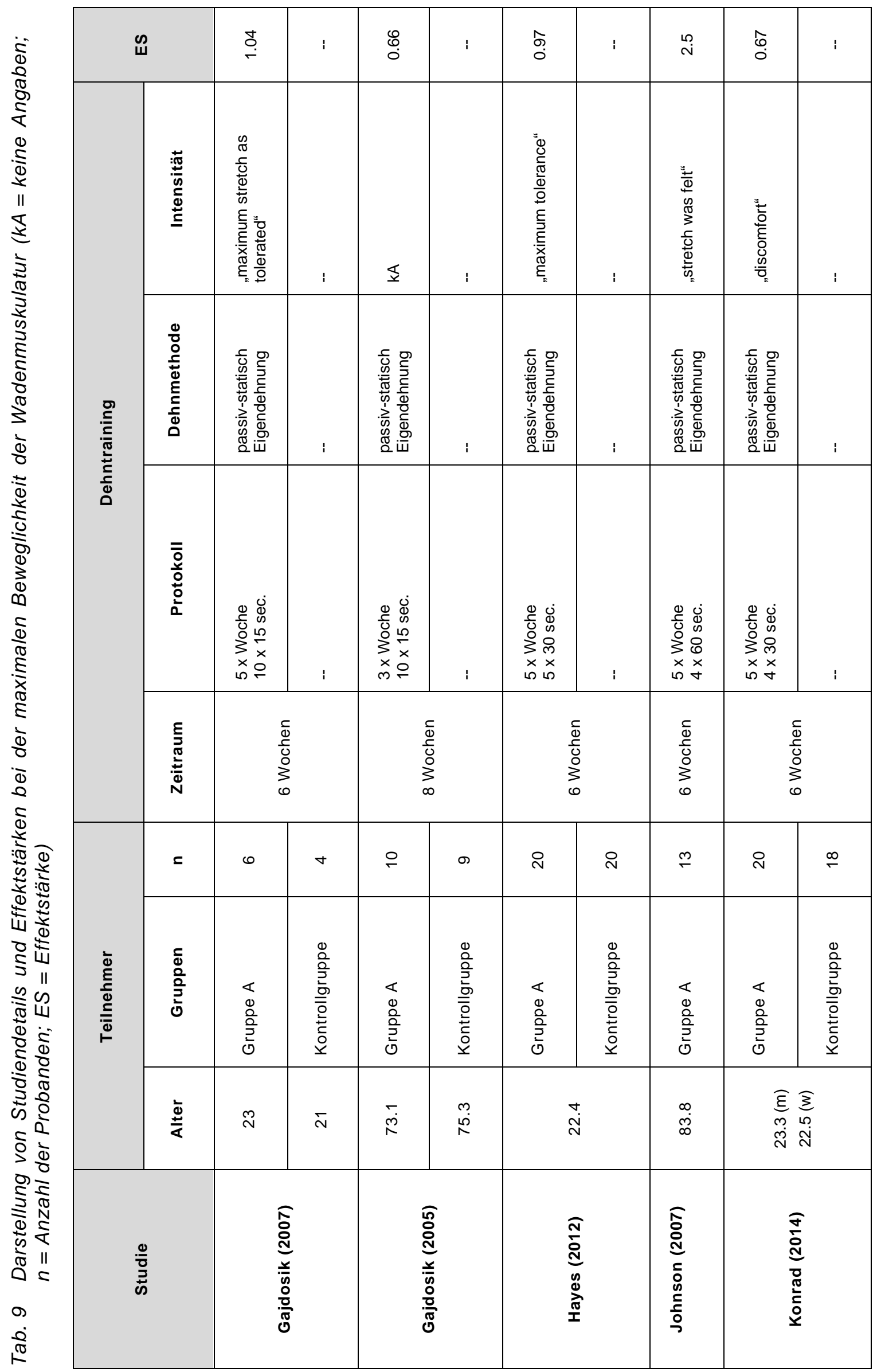




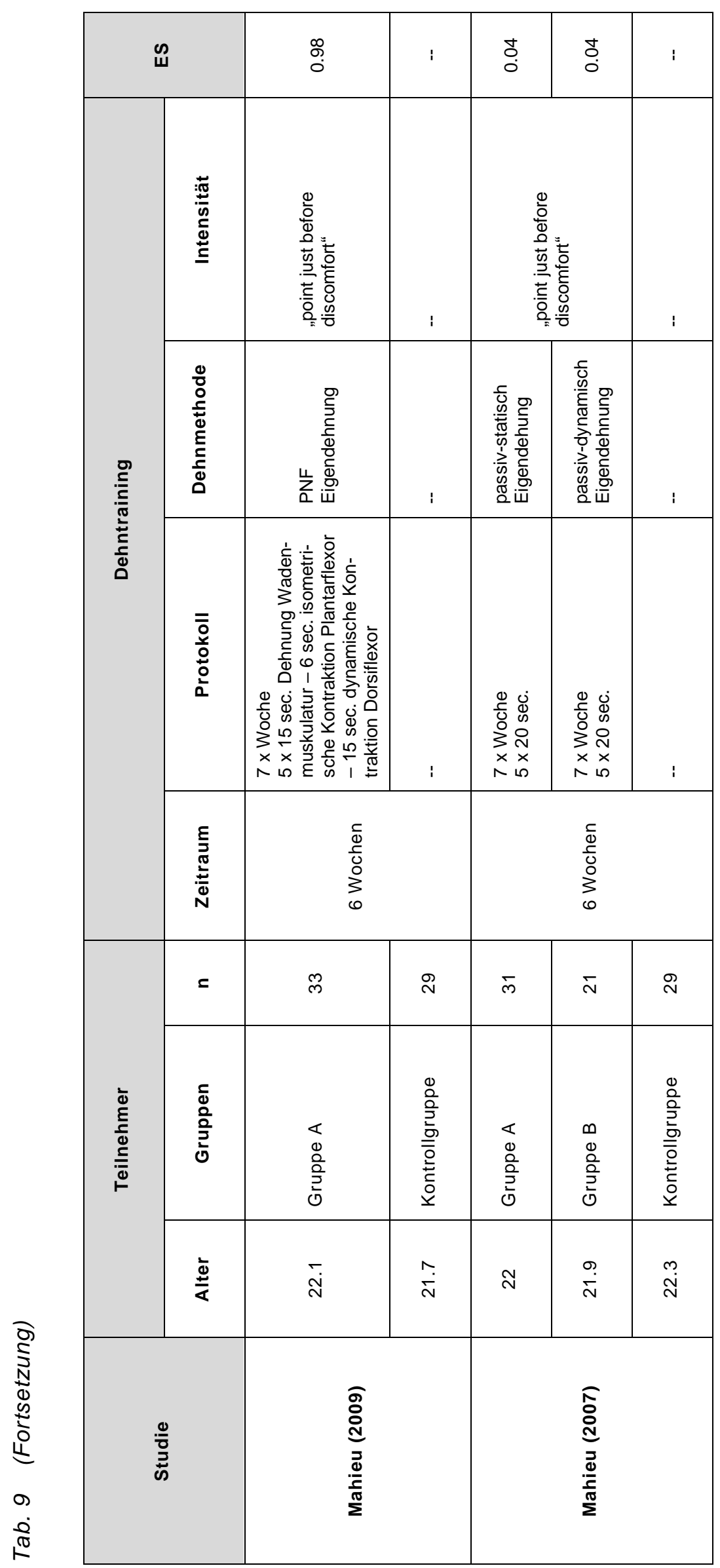




\begin{tabular}{|c|c|c|c|c|c|c|c|c|c|c|}
\hline \multicolumn{2}{|c|}{ 吕 } & $\underset{\sim}{\stackrel{N}{\sim}}$ & 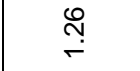 & : & $\frac{m}{0}$ & : & $\stackrel{\stackrel{\leftrightarrow}{\infty}}{\stackrel{\infty}{\infty}}$ & i & $\begin{array}{c}\infty \\
\substack{\infty \\
0}\end{array}$ & ; \\
\hline \multirow{4}{*}{ 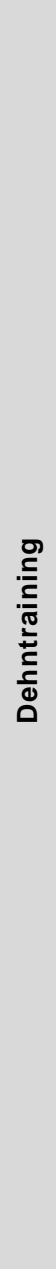 } & 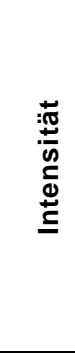 & 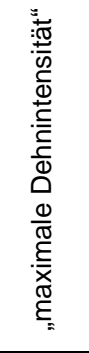 & 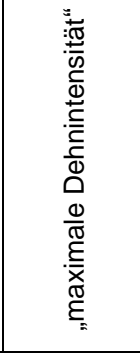 & : & 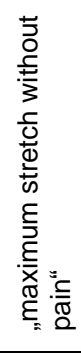 & : & 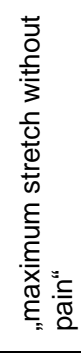 & : & 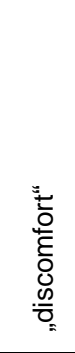 & : \\
\hline & 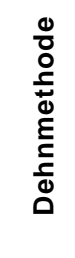 & 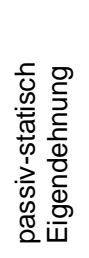 & 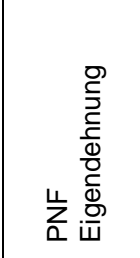 & : & 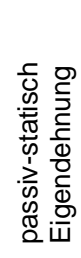 & : & 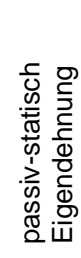 & : & 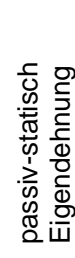 & : \\
\hline & $\begin{array}{l}\overline{\overline{0}} \\
\frac{8}{0} \\
\frac{0}{0} \\
\frac{0}{0}\end{array}$ & 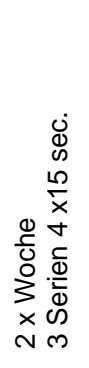 & 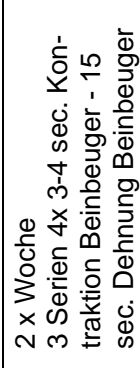 & : & 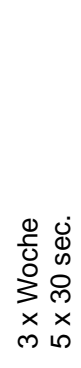 & : & 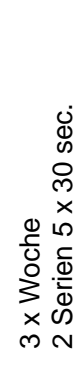 & : & 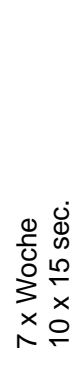 & : \\
\hline & 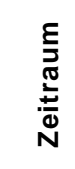 & \multicolumn{3}{|c|}{$\begin{array}{l}5 \\
0 \\
\frac{5}{0} \\
0 \\
3 \\
0\end{array}$} & \multicolumn{2}{|c|}{$\begin{array}{l}\frac{c}{2} \\
0 \\
\frac{1}{0} \\
\sum^{2} \\
\infty\end{array}$} & \multicolumn{2}{|c|}{$\begin{array}{l}\check{D} \\
\frac{1}{0} \\
\frac{1}{0} \\
\vdots \\
\vdots \\
\forall\end{array}$} & \multicolumn{2}{|c|}{\begin{tabular}{l}
\multicolumn{1}{c}{} \\
$\frac{1}{0}$ \\
$\frac{0}{0}$ \\
$\vdots$ \\
$m$
\end{tabular}} \\
\hline \multirow{3}{*}{ 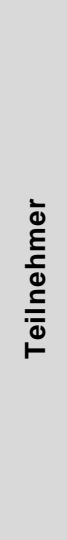 } & = & $\stackrel{\nabla}{ \pm}$ & $\stackrel{\nabla}{\square}$ & 0 & 으 & $\stackrel{ }{\circ}$ & 우 & 우 & $\stackrel{\simeq}{\simeq}$ & $\stackrel{N}{\sim}$ \\
\hline & 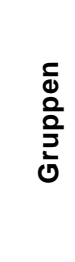 & $\begin{array}{l}\varangle \\
0 \\
00 \\
\frac{0}{2} \\
\frac{2}{0}\end{array}$ & $\begin{array}{l}\infty \\
0 \\
00 \\
\frac{0}{2} \\
\frac{1}{0}\end{array}$ & 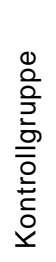 & $\begin{array}{l}\varangle \\
0 \\
00 \\
\frac{0}{2} \\
\frac{2}{0}\end{array}$ & 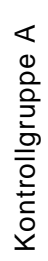 & $\begin{array}{l}\infty \\
0 \\
00 \\
0 \\
\frac{0}{3} \\
0\end{array}$ & $\begin{array}{l}0 \\
0 \\
0 \\
0 \\
0 \\
0 \\
0 \\
\overline{0} \\
0 \\
0 \\
0 \\
0 \\
x\end{array}$ & $\begin{array}{l}\varangle \\
0 \\
00 \\
\frac{0}{2} \\
\frac{3}{0}\end{array}$ & 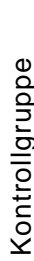 \\
\hline & 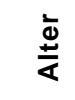 & & 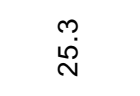 & & $\hat{\bar{N}}$ & 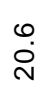 & 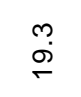 & $\overline{\mathrm{N}}$ & $\stackrel{m}{\stackrel{m}{N}}$ & ڤึ \\
\hline & & & 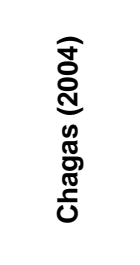 & & & & & & & \\
\hline
\end{tabular}




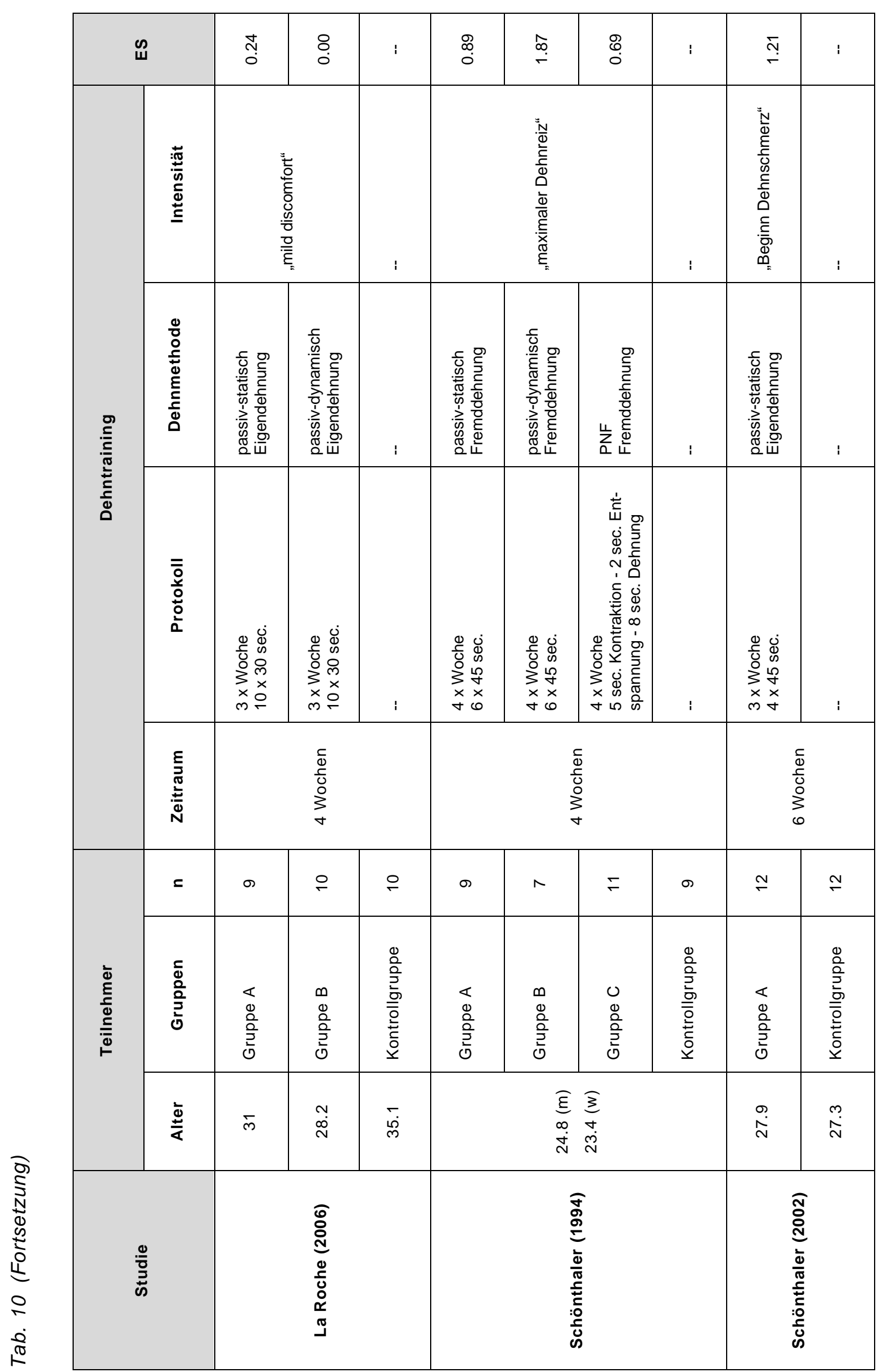




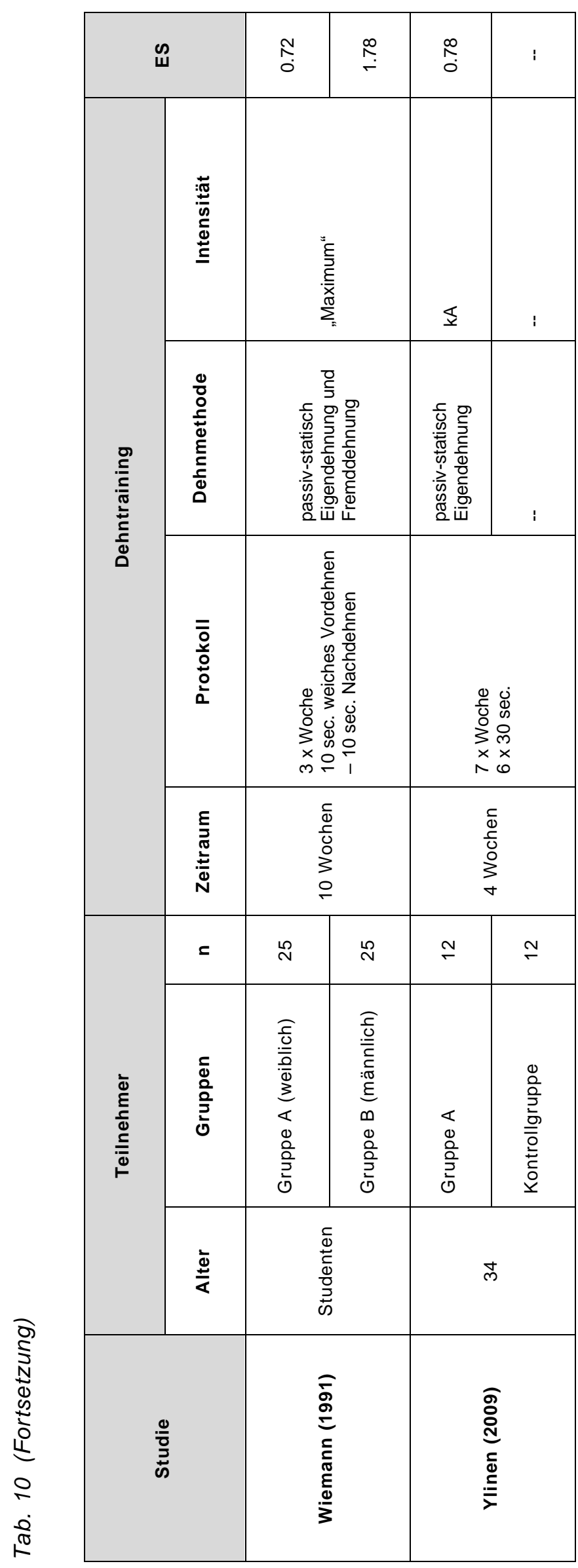




\begin{tabular}{|c|c|c|c|c|c|c|c|c|c|}
\hline \multicolumn{2}{|c|}{ 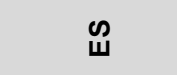 } & $\begin{array}{l}\infty \\
\stackrel{0}{0} \\
0\end{array}$ & : & $\underset{\leftrightarrow}{\stackrel{+}{\circ}}$ & : & 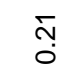 & : & $\frac{m}{0}$ & : \\
\hline \multirow{4}{*}{ 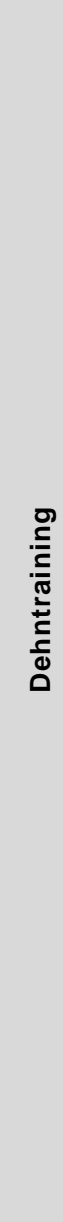 } & $\begin{array}{l}\frac{\pi}{\pi} \\
\frac{\pi}{0} \\
\stackrel{0}{ \pm} \\
\underline{\Xi}\end{array}$ & $\$$ & : & 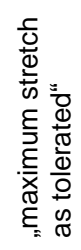 & : & 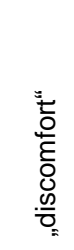 & : & 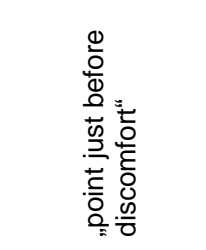 & : \\
\hline & 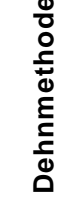 & 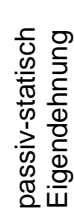 & : & 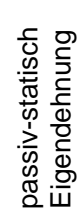 & : & 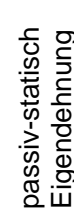 & : & 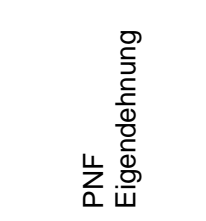 & : \\
\hline & 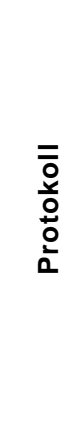 & 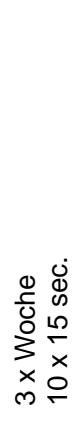 & : & 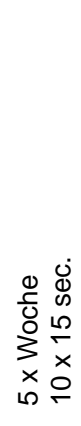 & : & 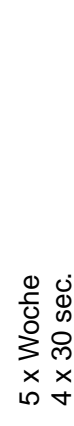 & : & 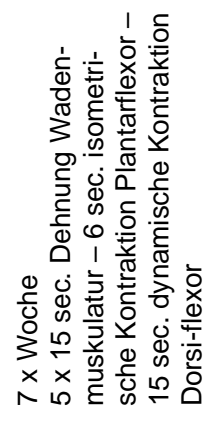 & : \\
\hline & 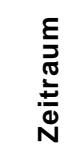 & & \multicolumn{2}{|c|}{$\begin{array}{l}\frac{1}{0} \\
\frac{1}{0} \\
0 \\
3 \\
0\end{array}$} & \multicolumn{2}{|c|}{$\begin{array}{l}\bar{\sigma} \\
\overline{0} \\
0 \\
3 \\
0\end{array}$} & \multicolumn{2}{|l|}{$\begin{array}{l}\bar{\sigma} \\
\bar{\delta} \\
0 \\
3 \\
0\end{array}$} \\
\hline \multirow{3}{*}{ 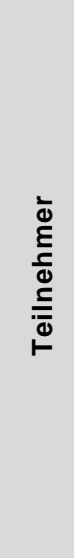 } & $=$ & 음 & $\sigma$ & 0 & $\nabla$ & ㅇ & $\stackrel{\infty}{\sim}$ & ల్ల & Dి \\
\hline & 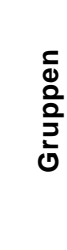 & $\begin{array}{l}\varangle \\
\searrow \\
\frac{0}{2} \\
\frac{2}{2} \\
0 \\
0\end{array}$ & 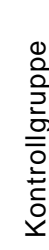 & 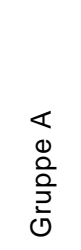 & 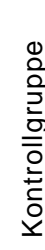 & $\begin{array}{l}\varangle \\
0 \\
\frac{0}{2} \\
\frac{2}{3} \\
0\end{array}$ & 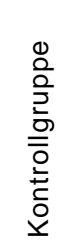 & $\begin{array}{l}\varangle \\
\searrow \\
\frac{0}{2} \\
\frac{2}{2} \\
0 \\
0\end{array}$ & 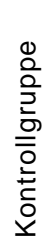 \\
\hline & $\frac{\grave{\Phi}}{\frac{ \pm}{4}}$ & $\bar{M}$ & مٌ & $\stackrel{\sim}{\sim}$ & $\bar{\sim}$ & & & $\overline{\mathrm{N}}$ & $\hat{\bar{N}}$ \\
\hline & & & & & & & & 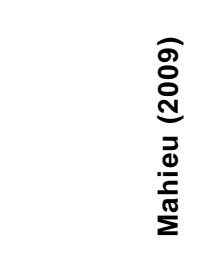 & \\
\hline
\end{tabular}




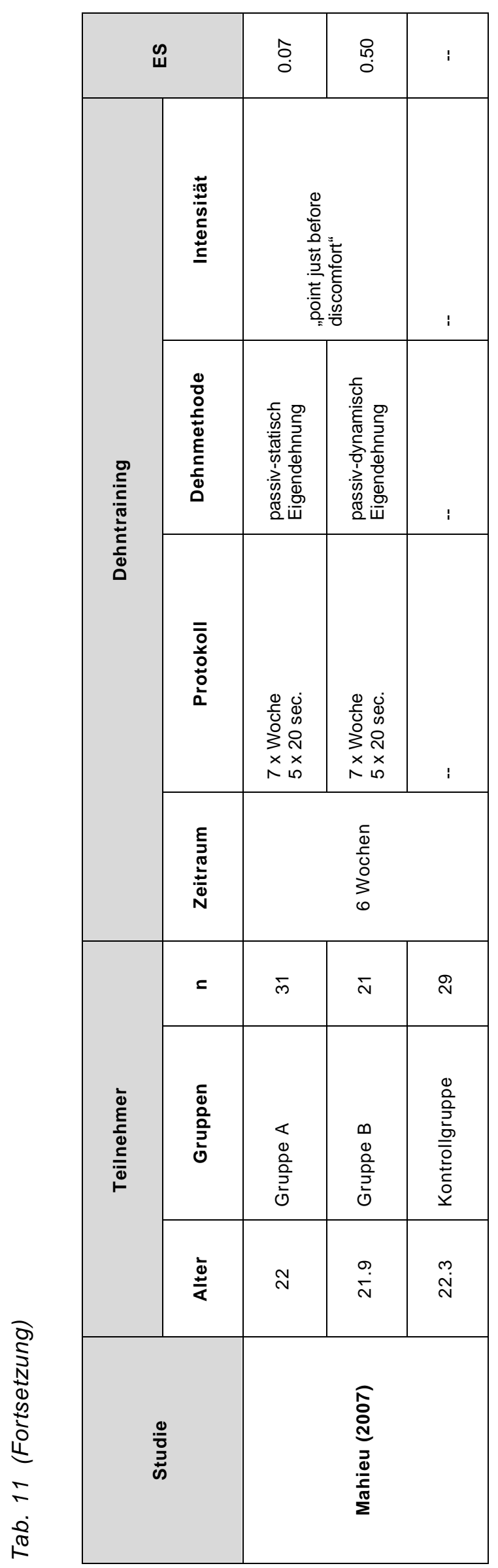




\subsubsection{Maximale Beweglichkeit}

Bei allen 83 Versuchsgruppen konnten Effektstärken zur Veränderung der maximalen Beweglichkeit berechnet werden. Sowohl die jüngeren $\left(E S_{\text {jung }}=1.3 \pm 0.8\right)$ als auch die älteren Erwachsenen $\left(E S_{\text {alt }}=0.8 \pm 0.7\right)$ erreichten nach der Definition von Cohen (1992) einen großen Effekt durch ein Dehntraining auf die maximale Beweglichkeit. Die Effektstärken unterschieden sich nicht signifikant zwischen den Altersgruppen $(t(81)=1.9, p=.06)$.

Die Dehnmethoden verteilten sich zu $12 \%$ auf ein aktiv-statisches Dehnen $\left(n_{V G-j u n g}=9, \quad n_{V G-a l t}=1\right), \quad$ zu $2 \%$ auf ein aktiv-dynamisches Dehnen $\left(n_{V G-j u n g}=2\right)$, zu $65.1 \%$ auf ein passiv-statisches Dehnen ( $n_{V G-j u n g}=46$, $\left.n_{V G-a l t}=8\right)$, zu $6 \%$ auf ein passiv-dynamisches Dehnen $\left(n_{V G-j u n g}=4, n_{V G-a l t}=1\right)$ und zu $14 \%$ auf die PNF-Methoden ( $\left.n_{V G \text {-jung }}=10, n_{V G-a l t}=2\right)$. Bei den Dehnmethoden konnte zwischen den Altersgruppen kein signifikanter Unterschied hinsichtlich der Effektstärken festgestellt werden $(F(1,74)=1.39, p=.24)$.

In $72 \%\left(n_{V G-j u n g}=53, n_{V G-a l t}=7\right)$ aller Versuchsgruppen haben sich die Probanden im Treatment selbst gedehnt (Eigendehnung) und bei $25 \%$ $\left(n_{V G-j u n g}=16, n_{V G-a l t}=5\right)$ wurden die Probanden extern gedehnt (Fremddehnung). Bei $2 \%$ der Versuchsgruppen $\left(n_{V G-j u n g}=2\right)$ wurde sowohl eine Eigenals auch eine Fremddehnung durchgeführt. Die Effektstärken der ROM älterer und jüngerer Erwachsener unterschieden sich unabhängig von der Dehnungsform (Eigen-Fremddehnung) signifikant voneinander $(F(1,77)=6.57, p<.05)$. Die Effektstärken der ROM in Abhängigkeit von der Art der Dehnung (Eigenund Fremddehnung) unterschieden sich unabhängig von der Altersgruppe nicht signifikant voneinander $(F<1.0, p=.8)$. Eine Wechselwirkung zwischen den Faktoren Altersgruppe und Dehnungsform war nicht signifikant $(F(1,77)=3.4$, $p=.07)$. Sowohl bei einer Eigen- als auch bei einer Fremddehnung erreichten die Probanden mittlere bis hohe Effektstärken (Tab. 12). 
Die Dehnintensitäten während eines Trainings wurden zu $33 \%$ als maximal ${ }^{5}$ $\left(n_{V G-j u n g}=24, n_{V G-a l t}=3\right)$ und zu $46 \%$ als submaximal ${ }^{6}\left(n_{V G-j u n g}=33, n_{V G-a l t}=5\right)$ angegeben. Bei $21.7 \%$ der Versuchsgruppen wurden keine Angaben zu den Trainingsintensitäten gemacht $\left(n_{V G-j u n g}=14, n_{V G \text {-alt }}=4\right)$. Die Effektstärken der ROM älterer und jüngerer Erwachsener unterschieden sich unabhängig von der Dehnintensität im Training (maximal-submaximal) nicht signifikant voneinander $(F(1,61)=2.1, p=.15)$. Die Effektstärken der ROM in Abhängigkeit von der Dehnintensität im Training unterschieden sich unabhängig von der Altersgruppe nicht signifikant voneinander $(F(1,61=1.5, p=.23)$. Eine Wechselwirkung zwischen den Faktoren Altersgruppe und Dehnintensität im Training war sign ifikant $(F(1,61)=5.4, p<.05)$. Dies weist daraufhin, dass sich Dehnintensitäten je nach Alter unterschiedlich auswirken. Jüngere profitieren stärker von maximalen Intensitäten, während Ältere eher von submaximalen Intensitäten profitieren. Bei den älteren Erwachsenen wurden mit einer maximalen Dehnintensität geringe und bei submaximaler Dehnintensität große Effektstärken erzielt (Tab. 12).

Die Empfehlungen zur Durchführung eines Dehntrainings hinsichtlich der Dauer einer Muskeldehnung, der Trainingshäufigkeit sowie der Dauer eines Dehntrainings auf altersspezifische Unterschiede werden nachfolgend überprüft. Nach Bandy, Irion und Briggler (1997) sowie Pollock et al. (1998) sollte ein Muskel 10 bis 30 Sekunden und bei älteren Erwachsenen bis zu 60 Sekunden gedehnt werden. Es wurde eine Kategorisierung der Dehnungsdauer der passivstatischen Dehnmethode kleiner/gleich 30 Sekunden und größer als 30 Sekunden vorgenommen. Die Effektstärken der ROM älterer und jüngerer Erwachsener unterschieden sich unabhängig von der Dauer einer Muskeldehnung nicht signifikant voneinander $(F<1, p=.6)$. Die Effektstärken der ROM in Abhängigkeit von der Dauer einer Muskeldehnung unterschieden sich unabhängig von der Altersgruppe nicht signifikant voneinander $(F<1, p=.8)$. Die Wechselwir-

5 Beispiel für maximale Dehnintensität: „maximal tolerable stretch“ (Hayes et al., 2012); „point of maximal tolerance" (LaRoche \& Connolly, 2006)

${ }^{6}$ Beispiel für submaximale Dehnintensität: „point of discomfort“ (Yuktasir \& Kaya, 2009); „maximum stretching tension without pain“ (Batista et al., 2009) 
kung zwischen den Faktoren Altersgruppe und Dauer einer Muskeldehnung war ebenso nicht signifikant $(F(1,50)=2.2, p=.15)$. Sowohl bei kleiner/gleich als auch bei länger als 30-sekündiger Muskeldehnung erreichten die Probanden mittlere bis hohe Effektstärken (Tab. 12).

Im Hinblick auf die Empfehlung, dass ein Dehntraining mindestens zwei- bis dreimal pro Woche durchgeführt werden sollte (Decoster et al., 2005), wurde eine Kategorisierung der Dehnhäufigkeit in kleiner/gleich dreimal pro Woche und größer als dreimal pro Woche vorgenommen. Die Effektstärken der ROM älterer und jüngerer Erwachsener unterschieden sich unabhängig von der Häufigkeit eines Dehntrainings nicht signifikant voneinander $(F(1,79)=3.8$, $p=.06$ ). Die Effektstärken der ROM in Abhängigkeit von der Häufigkeit eines Dehntrainings unterschieden sich unabhängig von der Altersgruppe nicht signifikant voneinander $(F(1,79)=2.5, p=.12)$. Eine Wechselwirkung zwischen den Faktoren Altersgruppe und der Häufigkeit eines Dehntrainings war nicht signifikant $(F<1, p=.91)$. Sowohl bei kleiner/gleich als auch bei mehr als dreimal Dehntraining pro Woche erreichten die Probanden mittlere bis hohe Effektstärken (Tab. 12).

Nach einer Empfehlung des American College of Sports Medicine sollte ein Dehntraining mindestens drei bis vier Wochen lang durchgeführt werden (Garber et al., 2011). Es wurde eine Kategorisierung der Treatmentdauer in kleiner/gleich und größer als vier Wochen vorgenommen. Die Effektstärken der ROM älterer und jüngerer Erwachsener unterschieden sich unabhängig von der Dauer eines Dehntrainings signifikant voneinander $(F(1,79)=4.8, p<.05)$. Die Effektstärken der ROM in Abhängigkeit von der Dauer eines Dehntrainings unterschieden sich unabhängig von der Altersgruppe nicht signifikant voneinander $(F<1, p=.7)$. Die Wechselwirkung zwischen den Faktoren Altersgruppe und Dauer eines Dehntrainings war nicht signifikant $(F(1,79)=1.4, p=.25)$. Sowohl bei einer kürzeren als auch einer länger andauernden Dehnungsintervention erreichten die Probanden mittlere bis hohe Effektstärken (Tab. 12). 
Tab. 12 Ergebnisse der ANOVA und Darstellung der Effektstärken durch die Veränderung der ROM in Abhängigkeit von Merkmalen eines Dehntrainings ( $n s=$ nicht signifikant; ${ }^{*}=$ Dauer einer Muskeldehnung bezogen auf die passiv-statische Dehnmethode) (mod. nach Haab, Martini, et al., 2017)

\begin{tabular}{|c|c|c|c|c|c|c|}
\hline Merkmal & Faktorstufen & $E_{\text {jung }}$ & ES $_{\text {alt }}$ & ANOVA & $\mathbf{F}$ & p \\
\hline \multirow{3}{*}{$\begin{array}{c}\text { (Faktor 1) } \\
\text { Dehnungsform }\end{array}$} & \multirow{2}{*}{ Fremddehnung } & \multirow{2}{*}{$1.7 \pm 1.1$} & \multirow{2}{*}{$0.6 \pm 0.5$} & Alter & 6.6 & $<.05$ \\
\hline & & & & Faktor 1 & $<1$ & ns \\
\hline & Eigendehnung & $1.2 \pm 0.7$ & $1.0 \pm 0.8$ & $\begin{array}{l}\text { Alter x } \\
\text { Faktor } 1\end{array}$ & 3.4 & ns \\
\hline \multirow{3}{*}{$\begin{array}{c}\text { (Faktor } 2) \\
\text { Dehnintensität }\end{array}$} & \multirow{2}{*}{ maximal } & \multirow{2}{*}{$1.4 \pm 1.0$} & \multirow{2}{*}{$0.2 \pm 0.2$} & Alter & 2.1 & ns \\
\hline & & & & Faktor 2 & 1.5 & ns \\
\hline & submaximal & $1.1 \pm 0.7$ & $1.4 \pm 0.7$ & $\begin{array}{l}\text { Alter x } \\
\text { Faktor } 2\end{array}$ & 5.4 & $<.05$ \\
\hline \multirow{3}{*}{$\begin{array}{c}\text { (Faktor 3) } \\
\text { Dauer einer } \\
\text { Muskeldehnung }\end{array}$} & \multirow{2}{*}{$\leq 30 \mathrm{sec}$} & \multirow{2}{*}{$1.4 \pm 0.9$} & \multirow{2}{*}{$0.7 \pm 0.3$} & Alter & $<1$ & ns \\
\hline & & & & Faktor 3 & $<1$ & ns \\
\hline & $>30 \mathrm{sec}$ & $0.8 \pm 0.3$ & $1.2 \pm 1.3$ & $\begin{array}{l}\text { Alter x } \\
\text { Faktor } 3\end{array}$ & 2.2 & ns \\
\hline \multirow{3}{*}{$\begin{array}{c}\text { (Faktor } 4) \\
\text { Häufigkeit eines } \\
\text { Dehntrainings }\end{array}$} & \multirow{2}{*}{$\leq 3$ pro Woche } & \multirow{2}{*}{$1.5 \pm 0.9$} & \multirow{2}{*}{$1.0 \pm 0.6$} & Alter & 3.8 & ns \\
\hline & & & & Faktor 4 & 2.5 & ns \\
\hline & $>3$ pro Woche & $1.1 \pm 0.6$ & $0.6 \pm 0.9$ & $\begin{array}{l}\text { Alter x } \\
\text { Faktor } 4\end{array}$ & $<1$ & ns \\
\hline \multirow{3}{*}{$\begin{array}{c}\text { (Faktor 5) } \\
\text { Dauer eines } \\
\text { Dehntrainings }\end{array}$} & \multirow{2}{*}{$\leq 4$ Wochen } & \multirow{2}{*}{$1.4 \pm 1.1$} & \multirow{2}{*}{$0.5 \pm 0.6$} & Alter & 4.8 & $<.05$ \\
\hline & & & & Faktor 5 & $<1$ & ns \\
\hline & $>4$ Wochen & $1.2 \pm 0.8$ & $1.0 \pm 0.7$ & $\begin{array}{c}\text { Alter } \mathrm{x} \\
\text { Faktor } 5\end{array}$ & 1.4 & ns \\
\hline
\end{tabular}

\subsubsection{Maximale Dehnungsspannung}

Bei 20 Versuchsgruppen konnten Effektstärken zur Veränderung der maximalen Dehnungsspannung berechnet werden. Allerdings untersuchte lediglich eine Studie (Gajdosik et al., 2005) mit einer Versuchsgruppe Dehnungsspan- 
nungsparameter bei älteren Erwachsenen mit mittlerer Effektstärke $\left(E S_{\text {alt }}=0.7\right)$. Ebenso wurde eine mittlere Effektstärke bei jüngeren Erwachsenen berechnet $\left(E S_{j u n g}=0.8 \pm 0.6\right)$. Aufgrund der geringen Anzahl an Studien, die die maximale Dehnungsspannung bei älteren Erwachsenen erhoben haben, entfällt an dieser Stelle ein weitergehender altersspezifischer Vergleich.

\subsection{Diskussion}

Der systematische Review hatte das Ziel, Anpassungserscheinungen durch ein Dehntraining zwischen älteren und jüngeren Erwachsenen zu vergleichen. Es wurden anhand der Deskriptoren in vier Datenbanken 42 Primärstudien gefunden und nach einem a priori festgelegten Kodierschema ausgewertet. Von den 42 Primärstudien beschäftigten sich neun Studien mit 254 älteren Probanden in einem mittleren Lebensalter von $62.7 \pm 12.0$ Jahren und 33 Studien mit 1222 jüngeren Probanden in einem mittleren Lebensalter von $23.1 \pm 2.7$ Jahren. Aufgrund der hohen Anzahl unterschiedlicher Dehnungsinterventionen innerhalb einzelner Studien, erfolgte die Ergebnisdarstellung bezogen auf die einzelnen Versuchsgruppen. Als abhängige Variablen wurde das Erreichen einer maximalen Beweglichkeit $\left[{ }^{\circ}\right]$ sowie die maximale Dehnungsspannung $[\mathrm{N} ; \mathrm{Nm}]$ definiert.

\subsubsection{Maximale Beweglichkeit}

Alle 42 Primärstudien mit 83 Versuchsgruppen untersuchten nach einem Dehntraining die maximale Beweglichkeit. Sowohl bei den älteren als auch bei den jüngeren Erwachsenen wurden große Effektstärken berechnet, welche sich nicht zwischen den Altersgruppen unterschieden. Diese Ergebnisse decken sich mit denen kurzfristiger Dehnungsinterventionen. Ryan et al. (2014) zeigten nach einer kurzfristigen Muskeldehnung bei älteren Erwachsenen ähnliche Zuwachsraten hinsichtlich der maximalen Beweglichkeit wie ihre jüngeren Pendants. Wydra (2008, S. 1312) kam im Rahmen einer Reanalyse von Studien mit jüngeren und älteren Probanden zu dem Ergebnis, dass „,... bei jüngeren und älteren Personen die gleichen Zuwachsraten zu beobachten" sind. Wird die 
Trainingshäufigkeit über den Untersuchungszeitraum der Primärstudien berücksichtigt, dann fällt auf, dass die älteren Probanden im Mittel wesentlich mehr Trainingseinheiten durchführten als die jüngeren Probanden. Auch ist festzustellen, dass die älteren eine große Effektstärke erreichten, die allerdings wesentlich niedriger war als bei jüngeren Erwachsenen. Somit kann bereits festgehalten werden, dass bei einem Dehntraining mit älteren Erwachsenen Effektstärken in der gleichen Größenordnung wie bei jüngeren Erwachsenen hinsichtlich der ROM zu erwarten sind. Allerdings benötigen ältere Personen hierfür mehr Trainingseinheiten.

Die Betrachtung der Dehnungsform (Eigen-Fremddehnung) ergab keinen signifikanten Unterschied zwischen den Altersgruppen. Mit einer Fremddehnung bei älteren Erwachsenen wurden mittlere Effektstärken und mit einer Eigendehnung hohe Effektstärken erreicht. Bei den jüngeren Erwachsenen ergaben beide Dehnungsformen hohe Effektstärken. Die nicht signifikanten geringeren Effektstärken bei der Fremddehnung der älteren Erwachsenen lassen sich möglicherweise damit begründen, dass ältere Erwachsene einer Fremddehnung ängstlicher gegenüberstehen und sich vorsichtshalber nicht maximal dehnen lassen. Glück et al. (2002) untersuchten nach einer singulären Dehnmaßnahme den Unterschied zwischen Eigen- und Fremddehnung auf die maximal zu erreichende Beweglichkeit. Sie kamen zu dem Ergebnis, dass durch direkte Eigendehnung größere Gelenkwinkel als durch eine Fremddehnung erreicht werden und führen dies auf eine direkte kinästhetische Rückmeldung zurück. Da die zu dehnende Person ihre Handlung selbst regulieren kann, entsteht ein Gefühl der Sicherheit. Bei älteren Erwachsenen könnte es ebenfalls sein, dass sie bei einer Eigendehnung das Dehntraining effektiver gestalten können.

Im Rahmen einer Dehnungsintervention ist die Intensität nur schwer zu quantifizieren (Marschall, 1999). Sie ist stark von der individuellen Schmerztoleranzgrenze der Probanden abhängig. Jüngere Erwachsene erreichten sowohl bei maximal als auch bei submaximal durchgeführten Dehnungsinterventionen hohe Effektstärken. Bei älteren Erwachsenen wurden mit einer maximalen Dehn- 
intensität geringe und bei submaximaler Dehnintensität große Effektstärken erzielt. Eine Wechselwirkung zwischen den Faktoren Altersgruppe und Dehnintensität war signifikant. Die Effektstärkenberechnung hinsichtlich der maximalen Dehnintensität älterer Erwachsener beruht auf nur einer Studie von Wydra et al. (1991) mit drei Versuchsgruppen. Bei den Probanden handelte es sich um Patienten ohne orthopädische Beschwerden, die sich aufgrund von internistischen und psychovegetativen Erkrankungen in klinischer Behandlung befanden. Die Probanden wurden fremd gedehnt. Es könnte sein, dass die Probanden keine maximale Dehnung des Therapeuten zuließen und es dementsprechend zu einer geringeren Effektstärke kam. Gajdosik et al. (2005, S. 976) bevorzugen eine submaximale Dehnintensität, da die Autoren der Meinung sind, dass die maximale Beweglichkeit psycho-physiologisch determiniert sei und dementsprechend keinen mechanischen Endpunkt im Sinne einer maximalen Muskellänge darstellt. In keiner Primärstudie wurde versucht, die Intensität, beispielsweise anhand einer Schmerzskala, zu objektivieren. Deshalb kann nicht davon ausgegangen werden, dass sich jeder Proband mit der gleichen Intensität gedehnt hat. Insbesondere bei älteren Erwachsenen kann es sein, dass diese sich vorsichtiger und nicht so schmerzintensiv dehnen wie jüngere Erwachsene. Apostolopoulos, Metsios, Flouris, Koutedakis und Wyon (2015, S. 1) beschreiben in einem Review "... the intensity of stretching is relatively under-researched". Eine Möglichkeit für zukünftige Studien besteht in der Nutzung einer Intensitätsskala für die Muskeldehnung um somit Studien vergleichbarer zu gestalten (Freitas et al., 2015).

Bezüglich unterschiedlicher Handlungsempfehlungen für ein Dehntraining wurden Kategorisierungen hinsichtlich der Dauer einer Muskeldehnung sowie der Häufigkeit und der Dauer eines Dehntrainings vorgenommen. Ältere Erwachsene erreichten eine große Effektstärke bei einer Muskeldehnung länger als 30 Sekunden und eine mittlere Effektstärke bei einer Muskeldehnung kleiner/gleich 30 Sekunden. Dieses Ergebnis entspricht den Empfehlungen von Bandy at al. (1997) und Pollock et al. (1998), dass ein passiv-statisches Dehntraining bei älteren Erwachsenen länger als 30 Sekunden gehalten werden soll und deckt sich mit Ergebnissen von Feland, Myrer, Schultheis, Fellingham und 
Meason (2001). Jüngere Erwachsene erreichten insgesamt hohe Effektstärken. Dieses Ergebnis deckt sich mit einer Studie von Bandy und Irion (1994) mit einer vergleichbaren Altersgruppe. Hier wurde beschrieben, dass eine 30-sekündige Dehnungsdauer ebenso effektiv ist wie eine 60-sekündige Dehnungsdauer.

Decoster et al. (2005) empfehlen ein Dehntraining von zwei- bis dreimal pro Woche. Die älteren und jüngeren Erwachsenen erreichten bei kleiner/gleich dreimal pro Woche eine große Effektstärke. Die Jüngeren erreichten bei mehr als dreimal Dehntraining pro Woche eine große und die Älteren eine mittlere Effektstärke. Dieses Ergebnis lässt vermuten, dass die Trainingshäufigkeit bei mindestens dreimal pro Woche liegen sollte, es aber bei mehr Trainingseinheiten zu keinem signifikanten Mehreffekt kommt. Cipriani, Terry, Haines, Tabibnia und Lyssanova (2012) kamen in ihrer Untersuchung mit vier Versuchsgruppen und unterschiedlichen Trainingsprotokollen zu einem gleichen Ergebnis. Zwei Versuchsgruppen dehnten sich dreimal pro Woche, entweder einmal oder zweimal am Tag, und zwei Versuchsgruppen dehnten sich täglich einmal oder zweimal. In allen vier Versuchsgruppen wurden signifikante Verbesserungen der ROM erzielt und die vier Versuchsgruppen unterschieden sich in ihren Ergebnissen nicht voneinander.

Nach einer Empfehlung des American College of Sports Medicine sollte ein Dehntraining mindestens drei bis vier Wochen lang durchgeführt werden (Garber et al., 2011). Jüngere Erwachsene erreichten sowohl bei kleiner/gleich vier Wochen als auch bei mehr als vier Wochen Dehntraining große Effektstärken. Bei älteren Erwachsenen wurden bei einem Dehntraining kleiner/gleich vier Wochen mittlere Effektstärken und bei einem Dehntraining mehr als vier Wochen große Effektstärken berechnet. Dies bedeutet, dass ältere Erwachsene vermutlich einen längeren Zeitraum für Anpassungserscheinungen an ein Dehntraining benötigen als jüngere Erwachsene. Ältere Erwachsene müssen mehr Trainingseinheiten durchführen, um Effektstärken in der gleichen GröBenordnung zu erreichen wie jüngere Erwachsene. Frankel, Bean und Frontera (2006) bestätigen die Annahme, dass aufgrund altersbedingter Veränderungen Adaptationsprozesse bei älteren Personen langsamer ablaufen. 


\subsubsection{Maximale Dehnungsspannung}

Ryan et al. (2014, S. 6) stellten eine signifikante Korrelation $(r=0.7)$ zwischen der maximalen Beweglichkeit und der maximalen Dehnungsspannung fest. Da die Erreichung einer maximalen Beweglichkeit sehr stark vom subjektiven Schmerzempfinden abhängig ist, ist es sinnvoll, die passive Dehnungsspannung als eine abhängige Variable im Rahmen einer Dehnungsintervention anzusehen (Toft, Espersen, Kålund, Sinkjaer, \& Hornemann, 1989, S. 493). Zwölf Studien mit insgesamt 20 Versuchsgruppen untersuchten neben der maximalen Beweglichkeit zusätzlich die maximale Dehnungsspannung. Nur eine Studie mit einer Versuchsgruppe wurde mit älteren Erwachsenen durchgeführt (Gajdosik et al., 2005). Sowohl die Studie mit den älteren als auch die mit den jüngeren Teilnehmern erreichte bei der Veränderung der maximalen Dehnungsspannung nach einer Dehnungsintervention mittlere Effektstärken. Bei der Studie mit den älteren Erwachsenen handelte es sich um eine passiv-statische Dehnungsintervention an der Wadenmuskulatur. Werden die Effektstärken nur aufgrund einer passiv-statischen Dehnungsintervention der jüngeren Versuchsgruppe an der Wadenmuskulatur mit der Studie von Gajdosik et al. (2005) mit älteren Teilnehmern verglichen, so erreichen die jüngeren Erwachsenen im Mittel eine kleine Effektstärke $\left(E S_{\text {jung }}=0.4 \pm 0.5\right)$ und die älteren Erwachsenen eine mittlere Effektstärke $\left(E S_{\text {alt }}=0.7\right)$. Dieses Ergebnis lässt darauf schließen, dass ältere Erwachsene eher von einer passiv-statischen Dehnung profitieren als jüngere Erwachsene.

\subsubsection{Subanalyse weiterer biomechanischer Parameter}

Neben den für diesen systematischen Review primären abhängigen Variablen (maximale Beweglichkeit und maximale Dehnungsspannung) und deren altersspezifischen Vergleich, wurden in den Primärstudien weitere biomechanische Parameter gefunden. Diese wurden bisher in Dehntrainingsstudien nicht so häufig untersucht, werden aber dennoch im Folgenden dargestellt. Hierbei handelt es sich um muskelelastische Parameter: submaximale Dehnungsspannung, Stiffness und absorbierte passive-elastische Energie. Bisher existieren zu wenige Trainingsstudien mit älteren Erwachsenen, die die Anpassungser- 
scheinungen der genannten muskelelastischen Parameter zwischen jüngeren und älteren Erwachsenen konkret klären könnten. Aus diesem Grund wird anstatt eines altersspezifischen Vergleiches nachfolgend auf den statistischen Vergleich mit einer Kontrollgruppe eingegangen.

Bei sieben Versuchsgruppen wurde die submaximale Dehnungsspannung untersucht. Diese wurde bei einem konstanten Gelenkwinkel (Konrad \& Tilp, 2014; Mahieu et al., 2007, 2009; Wiemann, 1991) und bei einem ersten Dehngefühl (Ylinen et al., 2009) gemessen. Keine Studie untersuchte die submaximale Dehnungsspannung bei älteren Erwachsenen. Es konnte nach einem Dehntraining in keiner dieser Studien eine signifikante Veränderung im Vergleich zu einer Kontrollgruppe festgestellt werden.

Bei vier Versuchsgruppen wurde das Integral unter der Dehnungsspannungskurve (passive-elastische Energie) in einem vorher definierten Gelenkwinkelbereich (Gajdosik et al., 2007, 2005; LaRoche \& Connolly, 2006; Schönthaler \& Ohlendorf, 2002) und bei einer Versuchsgruppe bei Ansteuerung eines konstanten Gelenkwinkels (Schönthaler \& Ohlendorf, 2002) gemessen. Nur eine Studie untersuchte die passiv-elastische Energie bei älteren Erwachsenen (Gajdosik et al., 2005). Bei La Roche und Connolly (2006) sowie Schönthaler und Ohlendorf (2002) kamen die Untersuchungen zu keinem signifikanten Unterschied zwischen einer Interventions- und einer Kontrollgruppe. Hingegen fanden Gajdosik et al. $(2007,2005)$ einen signifikanten Unterschied. Die bisher vorliegenden Studien zeigen keinen eindeutigen Befund, was sicherlich auch daran liegt, dass die passive-elastische Energie unterschiedlich erhoben wurde. Aufgrund dieser Heterogenität sind die Daten nicht unmittelbar miteinander vergleichbar. La Roche und Connolly (2006) untersuchten einen Abschnitt der Fläche unter der Dehnungsspannungskurve in einem mittleren Gelenkwinkelbereich und Gajdosik et al. (2005) die Fläche unter der gesamten Dehnungsspannungskurve. Die Erhebung der passiven-elastischen Energie erfolgt in Abhängigkeit der Beweglichkeit und der Dehnungsspannung. Es werden demnach unterschiedliche Ergebnisse der passiven-elastischen Energie berechnet, je nachdem, ob eine Muskeldehnung bis zu einer submaximalen oder maximalen Bewegungsreichweite durchgeführt wird (Magnusson, 1998). 
Bei acht Versuchsgruppen wurde die Stiffness berechnet (Gajdosik et al., 2007, 2005; Konrad \& Tilp, 2014; LaRoche \& Connolly, 2006; Mahieu et al., 2007, 2009; Schönthaler \& Ohlendorf, 2002). Nur eine Studie untersuchte die Stiffness bei älteren Erwachsenen (Gajdosik et al., 2005). Es konnte nach einem Dehntraining in keiner dieser Studien eine signifikante Veränderung im Vergleich zu einer Kontrollgruppe festgestellt werden.

Die Aussagekraft dieses Reviews beschränkt sich, aufgrund der Einschlusskriterien der Literaturrecherche, auf Personen ohne orthopädische Erkrankungen. Die Beschränkung auf Primärstudien, in denen Untersuchungen an Probanden ohne orthopädische Erkrankungen durchgeführt wurden, war allerdings notwendig, um eine einigermaßen homogene Stichprobe der älteren Erwachsenen zu erhalten. Es ist nicht auszuschließen, dass insbesondere ältere Personen subtile degenerative Erkrankungen aufgewiesen haben könnten.

Abschließend soll noch auf die Effektstärke als Beurteilungsmaß für die praktische Relevanz der Untersuchungsergebnisse eingegangen werden. Die Bewertung der Ergebnisse in den Primärstudien erfolgte anhand der Klassifikation nach Cohen (1992). Zu beachten ist, dass diese Klassifikation lediglich eine Orientierungshilfe darstellt. „Letztlich entscheidet der jeweilige Untersuchungskontext darüber, was als kleiner oder als großer Effekt zu bezeichnen ist" (Bortz \& Döring, 2006, S. 626). Werden die wichtigsten Signifikanztests betrachtet, so kann beispielsweise das Ergebnis einer Varianzanalyse mit einer Effektstärke $>0.4$ als groß interpretiert werden (ebd., S. 606). Wie in einem Beitrag von Fröhlich und Pieter (2009, S. 142) beschrieben „... müssen die Effektstärken je nach Zielgruppe, Setting und Fragestellung unterschiedlich interpretiert werden". Insbesondere die Beweglichkeit stellt bei Personen in höherem Lebensalter eine wichtige Ressource dar, um eine adäquate Alltagsmotorik zu gewährleisten. Aus diesem Grund wird in einem höheren Lebensalter ein Dehntraining zur Verbesserung der Beweglichkeit empfohlen und die praktische Relevanz eines Dehntrainings bei älteren höher eingestuft als bei jüngeren Erwachsenen. 


\subsection{Zusammenfassung}

Mit dem systematischen Review und der anschließenden metaanalytischen Betrachtung wurden Anpassungserscheinungen durch ein Dehntraining bei jüngeren und älteren Erwachsenen anhand von 42 Primärstudien untersucht. Die abhängige Variable wurde zumeist eindimensional über die Erreichung der maximalen Beweglichkeit definiert. Sowohl jüngere als auch ältere Erwachsene erreichten große Effektstärken hinsichtlich der maximalen Beweglichkeit nach einer Dehnungsintervention. Es scheint so zu sein, dass ältere Erwachsene längere Trainingsphasen benötigen sowie eher von einer Eigendehnung und einer submaximalen Dehnintensität profitieren. Des Weiteren sollte die Dehndauer bei älteren Erwachsenen mit einer passiv-statischen Dehnung länger als 30 Sekunden dauern. Wenige Studien untersuchten die Anpassungserscheinungen mehrdimensional im Sinne von zusätzlichen biomechanischen Parametern wie zum Beispiel der maximalen Dehnungsspannung, der Stiffness oder der passiven-elastischen Energie. Nur eine Studie untersuchte die maximale Dehnungsspannung bei älteren im Vergleich zu jüngeren Erwachsenen. Da diese eine Studie mit älteren Erwachsenen ein passiv-statisches Dehntraining durchführte, wurden die Effektstärken der Studien mit jüngeren Erwachsenen, die ebenfalls ein passiv-statisches Dehntraining durchführten, verglichen. Es stellte sich heraus, dass Ältere eine mittlere und Jüngere eine kleine Effektstärke aufweisen. Aufgrund altersabhängiger unterschiedlicher Effektstärken scheinen Ältere besser auf ein passiv-statisches Dehntraining zu reagieren als Jüngere.

Es existieren bisher keine Befunde, in denen ältere und jüngere Probanden unter gleichen Bedingungen ein mehrwöchiges Dehntraining durchführten. Somit fehlt ein konkreter Vergleich von Anpassungserscheinungen zwischen jüngeren und älteren Erwachsenen. Für ältere Erwachsene wird ein Dehntraining empfohlen, wobei diese Empfehlungen auf wenige Studien zurückzuführen sind. Trainingsempfehlungen für ältere Erwachsene auf der Basis von Studien mit jüngeren Erwachsenen sind aufgrund von degenerativen Veränderungen in den Bereichen des Muskel- und Skelett-Systems nicht ohne Weiteres möglich. 
Aufgrund der Vielzahl an unterschiedlichen Dehnungsinterventionen lassen sich Trainingsstudien nur annähernd miteinander vergleichen. Es ist auf die nach wie vor defizitäre Forschungslage bei älteren Erwachsenen hinzuweisen. Derzeit überwiegen Studien mit jüngeren Erwachsenen, die darüber hinaus eher eindimensional ausgerichtet sind. Für ein besseres Verständnis über die Auswirkungen eines langzeitigen Dehntrainings sollten zukünftige Studien eher mehrdimensional ausgerichtet und mit älteren Erwachsenen durchgeführt werden. Dies bedeutet, dass neben der maximalen Beweglichkeit und der maximalen Dehnungsspannung auch die gesamte Dehnungsspannungskurve zur Ableitung einer gelenkwinkelabhängigen Muskelelastizität (Stiffness) oder aber auch die Fläche unter der Dehnungsspannungskurve als Maß für die passivelastische Energie herangezogen werden sollte. 


\section{Altersspezifische Anpassungen an ein Dehntraining: Eine empirische Untersuchung}

Aus dem vorangegangenen systematischen Review wurde ersichtlich, dass bei Trainingsstudien im Bereich der Muskeldehnung zumeist jüngere Probanden rekrutiert wurden. Untersuchungen zur Trainierbarkeit der Muskeldehnfähigkeit bei Personen in höherem Lebensalter fehlen vollständig. Des Weiteren konnte gezeigt werden, dass sich die meisten Trainingsstudien eindimensional primär mit der Messung der Beweglichkeit befassten. Ohne die zusätzliche Betrachtung der Dehnungsspannungskurve lassen sich allerdings keine Rückschlüsse auf die Veränderungen von passiven Eigenschaften der Muskulatur, im Sinne der Muskeldehnfähigkeit, ziehen.

Das Ziel dieser empirischen Untersuchung ${ }^{7}$ ist der Vergleich von Anpassungserscheinungen der ischiocruralen Muskulatur durch ein zehnwöchiges standardisiertes Dehntraining bei jüngeren und älteren Erwachsenen. In Anbetracht der vorangegangenen Literaturrecherche wird davon ausgegangen, dass das Ausgangsniveau zwischen den älteren und den jüngeren Probanden auf einem unterschiedlichen Niveau zugunsten der jüngeren Probanden sein wird, allerdings die Anpassungen an ein Dehntraining in einer ähnlichen Größenordnung liegen werden.

\subsection{Fragestellung}

Es soll die folgende Frage geklärt werden: Besteht ein Unterschied in der Trainierbarkeit der Muskeldehnfähigkeit zwischen jüngeren und älteren Erwachsenen?

7 Diese Untersuchung wurde zum Teil unter dem Titel „The effect of age on hamstring passive properties after a 10-week stretch training" im Journal of Physical Therapy Science im Juni 2017 veröffentlicht (Haab \& Wydra, 2017).

Erste Ergebnisse dieser Untersuchung wurden auf der 10. Jahrestagung der Deutschen Gesellschaft für Biomechanik im März 2017 vorgestellt (Haab, De Borger, \& Wydra, 2017). 


\subsection{Methodik}

Die vorliegende empirische Untersuchung, einschließlich der beschriebenen Vorstudien, entspricht der Deklaration von Helsinki und wurde durch die Ethikkommission der Fakultät 5, Empirische Humanwissenschaften, der Universität des Saarlandes geprüft und genehmigt (Antrag 15-5). Allen Versuchsteilnehmern wurde ein Informationsblatt vorgelegt, in dem der Ablauf der Untersuchung sowie Nutzen und Risiken beschrieben wurden. Die Teilnehmer unterschrieben eine Einverständniserklärung, mit der sie darauf hingewiesen wurden, dass die Teilnahme freiwillig ist und sie zu jeder Zeit von ihrer Teilnahme zurücktreten können. Die Weiterverarbeitung der Teilnehmerdaten sowie die erhobenen Untersuchungsparameter erfolgten anonymisiert, sodass im Nachhinein kein Rückschluss auf einzelne Teilnehmer vorgenommen werden konnte.

\subsubsection{Personenstichprobe}

Der erforderliche Stichprobenumfang wurde a priori mithilfe des Programms G*Power ermittelt (Faul, Erdfelder, Buchner, \& Lang, 2009). Die Festlegung der Stichprobengröße orientierte sich zudem an ähnlich durchgeführten experimentellen Studien (Gajdosik et al., 2005; Schönthaler \& Ohlendorf, 2002). Die Berechnung der Stichprobengröße basierte auf einem $\alpha$-Niveau von $p \leq .05$ und einer Power (1- $\beta$ ) von 0.8. Insgesamt wurden pro Versuchsgruppe (eine Gruppe ältere Erwachsene, eine Gruppe jüngere Erwachsene) zehn auswertbare Probanden benötigt. Um etwaige Drop-Outs zu berücksichtigen, wurden 15 jüngere und 14 ältere Erwachsene rekrutiert. Die jüngere Versuchsgruppe setzte sich aus Sportstudenten im vierten Semester zusammen. Die ältere Versuchsgruppe wurde aus einer Präventivsportgruppe der Universität des Saarlandes rekrutiert. Alle Versuchspersonen waren männlich und führten vor Untersuchungsbeginn kein regelmäßiges Dehntraining durch. Für beide Versuchsgruppen wurden folgende Ausschlusskriterien festgelegt:
a) Schmerzen in Hüft- und/oder Kniegelenk,
b) Schmerzen in der Muskulatur der Oberschenkelrückseite,
c) Gelenkprothese in Hüft- und/oder Kniegelenk, 
d) Rückenschmerzen,

e) aktuelle oder alte Verletzungen an Hüft- und/oder Kniegelenk,

f) aktuelle Verletzungen der Muskulatur an der Oberschenkelrückseite,

g) Sportarten mit hohem Anteil an Gelenkbeweglichkeit.

Weiterhin sollten die Probanden nicht mehr als 60 Minuten pro Tag und nicht mehr als drei Tage pro Woche sportlich aktiv sein. Bei diesem sportlichen Umfang gelten die Probanden in Anlehnung an Hoge et al. (2010) als Freizeitsportler. Die Probanden wurden außerdem darauf hingewiesen, ihre Lebensgewohnheiten während des Untersuchungszeitraumes beizubehalten und auf neue ungewohnte sportliche Aktivitäten zu verzichten. Nach Drop-Out beendeten 12 ältere (Alter: $65.1 \pm 7.9$ Jahre, Größe: $178.8 \pm 6.1 \mathrm{~cm}$, Gewicht: $81.9 \pm$ $8.0 \mathrm{~kg}$ ) und 13 jüngere Probanden (Alter: $24.0 \pm 4.0$ Jahre, Größe: $178.5 \pm$ $7.2 \mathrm{~cm}$, Gewicht: $75.9 \pm 10.1 \mathrm{~kg}$ ) die Untersuchung, die dann letztendlich in die statistischen Analysen eingingen. Die Gründe für den Drop-Out waren bei einem älteren und zwei jüngeren Probanden Krankheit und bei einem älteren Probanden mangelnde Compliance.

\subsubsection{Variablenstichprobe}

Zur Messung der Muskeldehnfähigkeit sind apparative Systeme zur zeitsynchronen Erfassung von Winkel-, Kraft- und EMG-Parameter notwendig. Neben kommerziellen Systemen (bspw. Isokinetische Dynamometer) existieren individuell angefertigte Messsysteme, die eine sensiblere Datenaufnahme gewährleisten (Buckner et al., 2015). Ein Vorteil solcher individuell angefertigten Messsysteme ist die Anpassung und Modifizierung an den aktuellen Forschungsstand. Um quantitative biomechanische und muskelphysiologische Daten zu erheben, wurde in der Vergangenheit eine stationäre Messapparatur entwickelt (Schönthaler et al., 1998), die für diese Untersuchung in modifizierter Form herangezogen wurde. Nachfolgend wird zunächst die modifizierte Messapparatur beschrieben und im Anschluss die damit erhobenen abhängigen Variablen erläutert. 


\subsubsection{Messapparatur}

Die nach Schönthaler et al. (1998) modifizierte Messapparatur wird im Folgenden als apparativer Straight-Leg-Raise-Test (ASLRT) bezeichnet (Abb. 20).

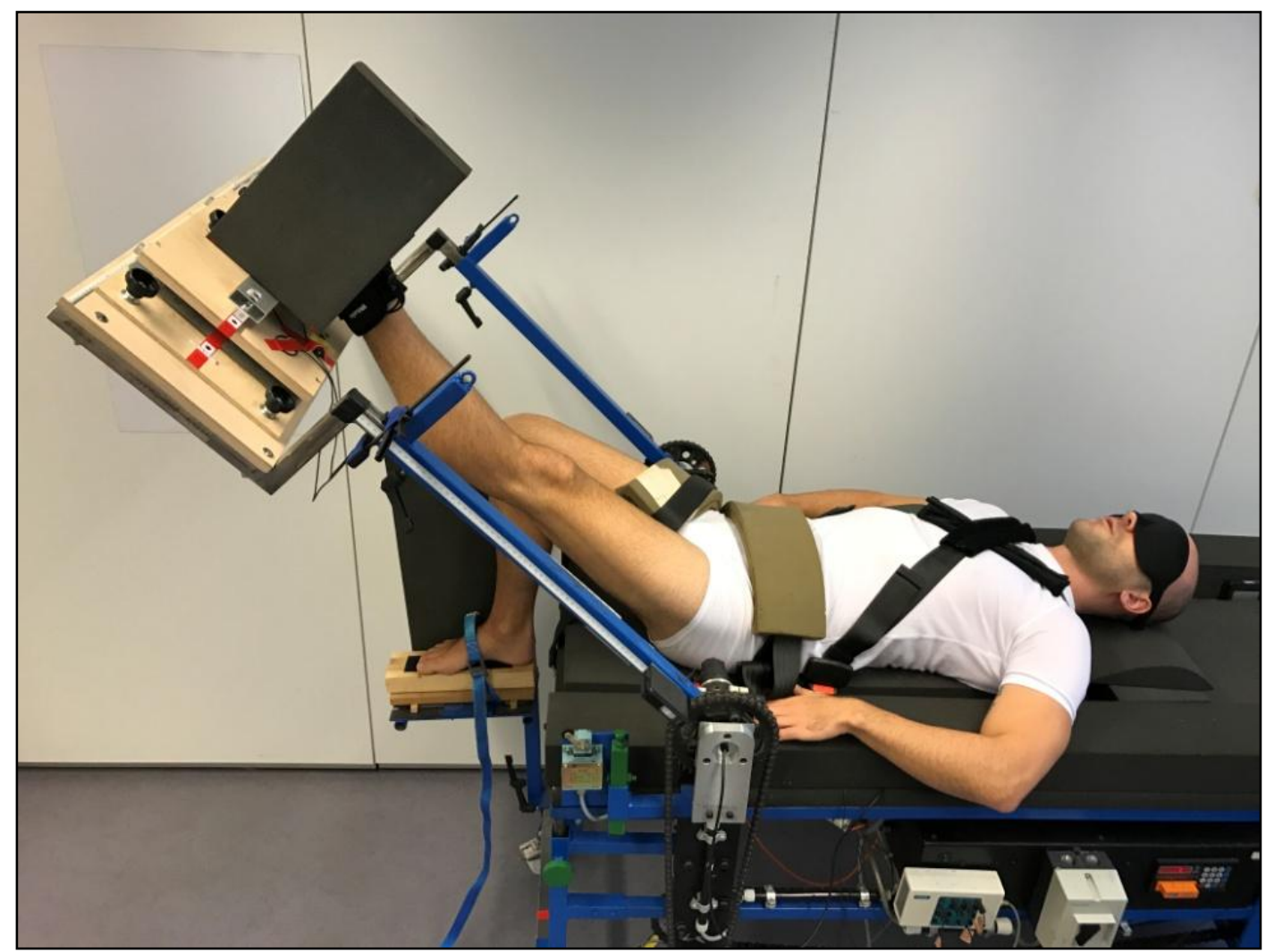

Abb. 20 Apparativer Straight-Leg-Raise-Test (ASLRT) (Haab \& Wydra, 2017, S. 1050)

Der ASLRT lässt eine standardisierte Dehnung der ischiocruralen Muskulatur bei fixiertem Hüft-, Bein- und Oberkörperbereich zu. Hierzu befanden sich die Probanden in Rückenlage auf dem ASLRT und das zu testende Bein wurde, im Kniegelenk gestreckt, auf einem Hebelarm in horizontaler Position abgelegt. Das Gegenbein wurde seitlich angestellt und im Leistenbereich fixiert, um eine Retroversion des Beckens während der Dehnung zu verhindern. Weiterhin wurde das Becken sowie der Oberkörper mit Gurten fixiert (Abb. 21). Um visuelle Ablenkungen auszuschließen, wurden die Augen der Probanden während der Dehnungsprozedur mit einer Schlafmaske abgedeckt. Der Dehnungsvorgang erfolgte über eine indirekte Eigendehnung der ischiocruralen Muskulatur und wurde durch einen Elektromotor, der eine konstante Dehnungsgeschwin- 
digkeit vorgibt, durchgeführt. Der Proband steuerte den Dehnungsvorgang über einen 1-Wege-Joystick (Saia-Burgess Electronics, Schweiz). Wurde der Joystick nach hinten (zum Probanden) bewegt, so wurde der Hebelarm mit einer Geschwindigkeit von $1.5 \% \mathrm{sec}$. nach oben gefahren. Diese langsame Geschwindigkeit sollte dazu führen, dass muskuläre Reflexe nicht aufgrund einer zu hohen Dehnungsgeschwindigkeit ausgelöst werden (Matthews, 1972). Wurde der Joystick losgelassen und befand sich somit in der Mittelstellung, dann blieb der Hebelarm in der jeweiligen Position stehen. Wurde der Joystick nach vorne gedrückt (vom Probanden weg), so bewegte sich der Hebelarm mit einer Geschwindigkeit von $3.75 \% \mathrm{sec}$. wieder herunter in Richtung der Ausgangsposition. Während der Dehnungsprozedur konnte jederzeit in den Dehnungsvorgang eingegriffen und der ASLRT per Notstopp-Schalter ausgeschaltet werden.

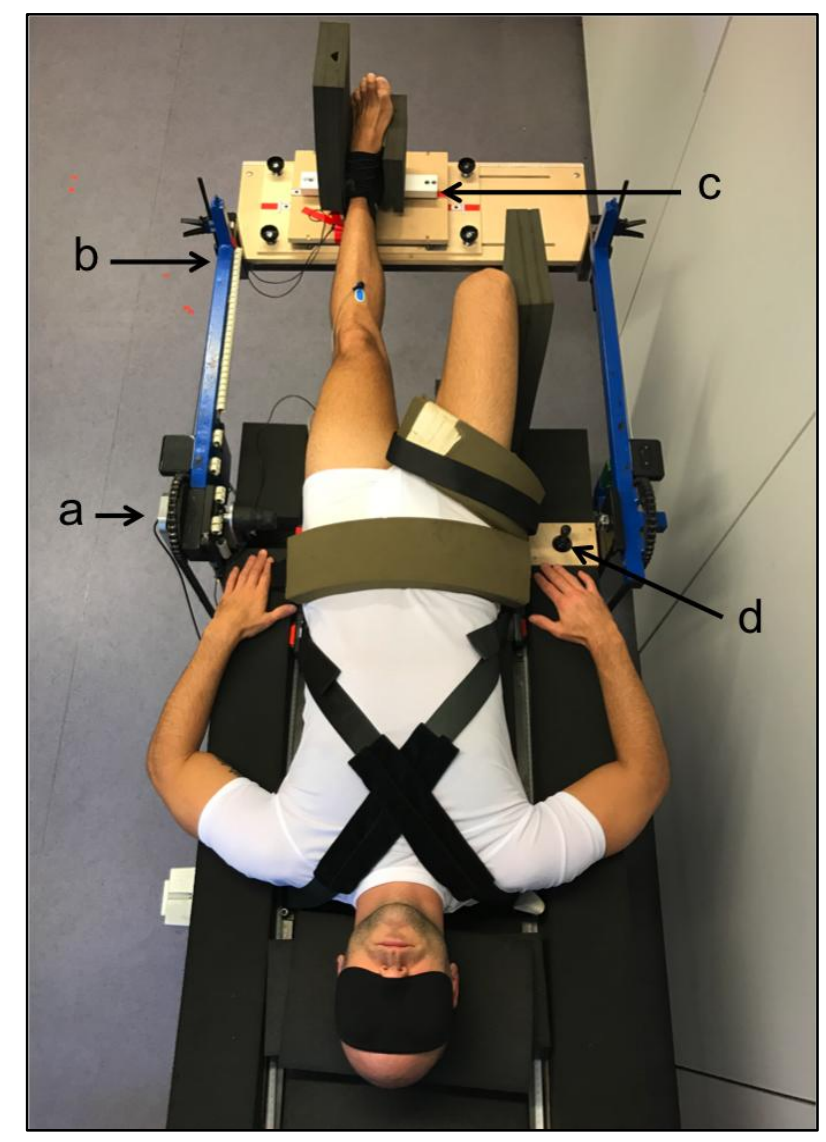

Abb. 21 ASLRT von oben ( $a=$ Potentiometer zur Messung der Beweglichkeit, $b=$ in der Länge verstellbarer Hebelarm, $c=$ Kraftmessplatte zur Messung der Dehnungsspannung, $d$ = Joystick zur Bedienung des Elektromotors) 
Modifiziert wurde die Messapparatur von Schönthaler et al. (1998), indem der Steuerungsvorgang am ASLRT intuitiver durch einen Joystick durchgeführt werden konnte. Vorher wurde das Hoch- und Herunterfahren des Hebelarms durch zwei Druckschalter gesteuert (Schönthaler \& Ohlendorf, 2002, S. 108). Weiterhin wurde zur Messung der Beweglichkeit ein neuer Drehpotentiometer (Biovision, Wehrheim) in der Drehachse des ASLRT verbaut. Neben diesen zwei kleineren Modifikationen wurde ein neues Messsystem zur Erfassung der Dehnungsspannung entwickelt, das im Folgenden als Kraftmessplatte bezeichnet wird. Die Kraftmessplatte wurde am Hebelarm des ASLRT montiert (Abb. 21, c). Die Vorüberlegungen zur Entwicklung einer für diesen Untersuchungsaufbau notwendigen Kraftmessplatte wird im Folgenden erläutert.

Während eines Dehnungsvorganges der ischiocruralen Muskulatur an einem ASLRT kommt es nach Schönthaler et al. (1998) zu einer Longitudinalverschiebung des Beines, da die anatomische Drehachse des Hüftgelenkes nicht mit der apparativen Drehachse des ASLRT übereinstimmt (Abb. 22). Die Longitudinalverschiebung kann das Verhalten des Probanden in der Testsituation erheblich beeinträchtigen und somit Testergebnisse beeinflussen (Nahler, 1998; Schönthaler et al., 1998). In einer Untersuchung von Nahler (1998) wurde deshalb versucht, über die Messung der Schritthöhe plus $8 \mathrm{~cm}$ eine Annäherung an die anatomische Drehachse zu finden, um eine Longitudinalverschiebung des Beines zu minimieren. Aus testökonomischer Sicht erscheint diese Vorgehensweise als ungünstig. Des Weiteren kann es dennoch, je nach anatomischen Voraussetzungen des Hüftgelenkes, zu einer Longitudinalverschiebung kommen. 


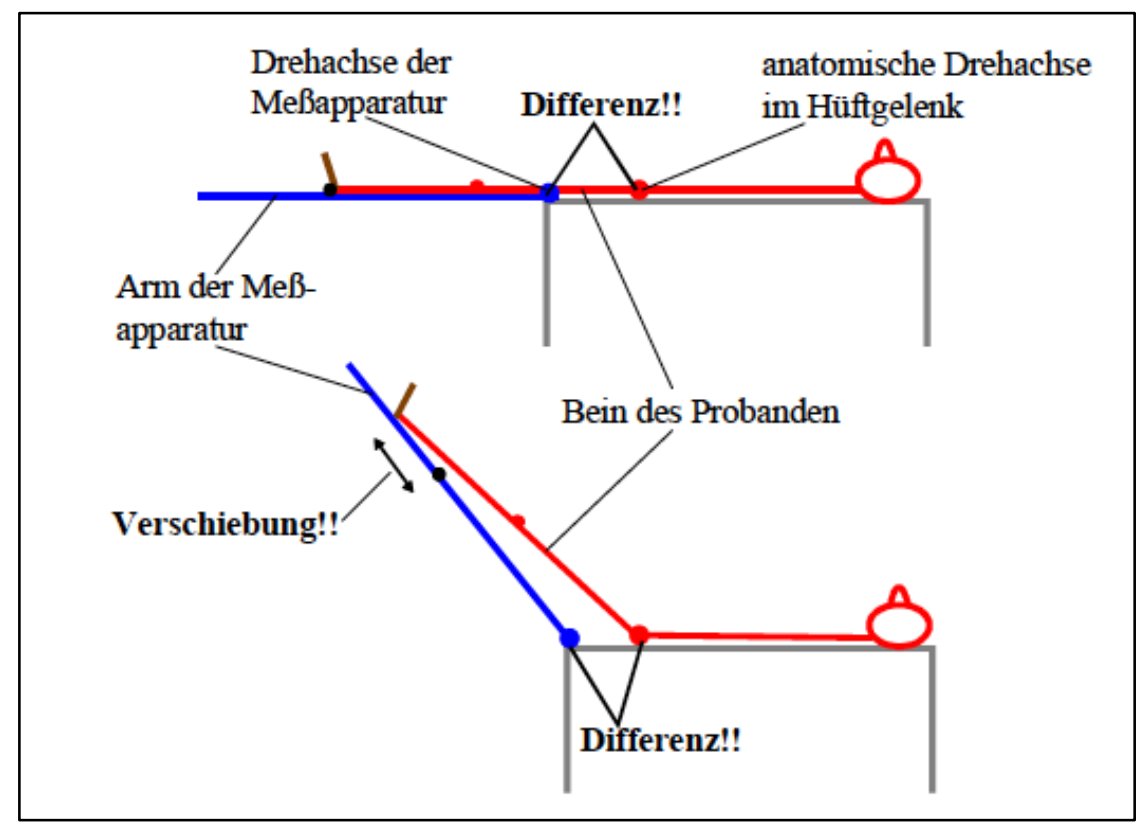

Abb. 22 Schematische Darstellung einer Longitudinalverschiebung während einer Dehnung auf dem ASLRT (Nahler, 1998, S. 6)

Ausgehend von dieser Problematik wurde eine Kraftmessplatte entwickelt, die es ermöglicht, eine Longitudinalverschiebung auszugleichen ohne die Messergebnisse zu beeinflussen. Um eine mögliche Longitudinalverschiebung auszugleichen, wurde die Kraftmessplatte auf Schienen gelagert (Abb. 23).

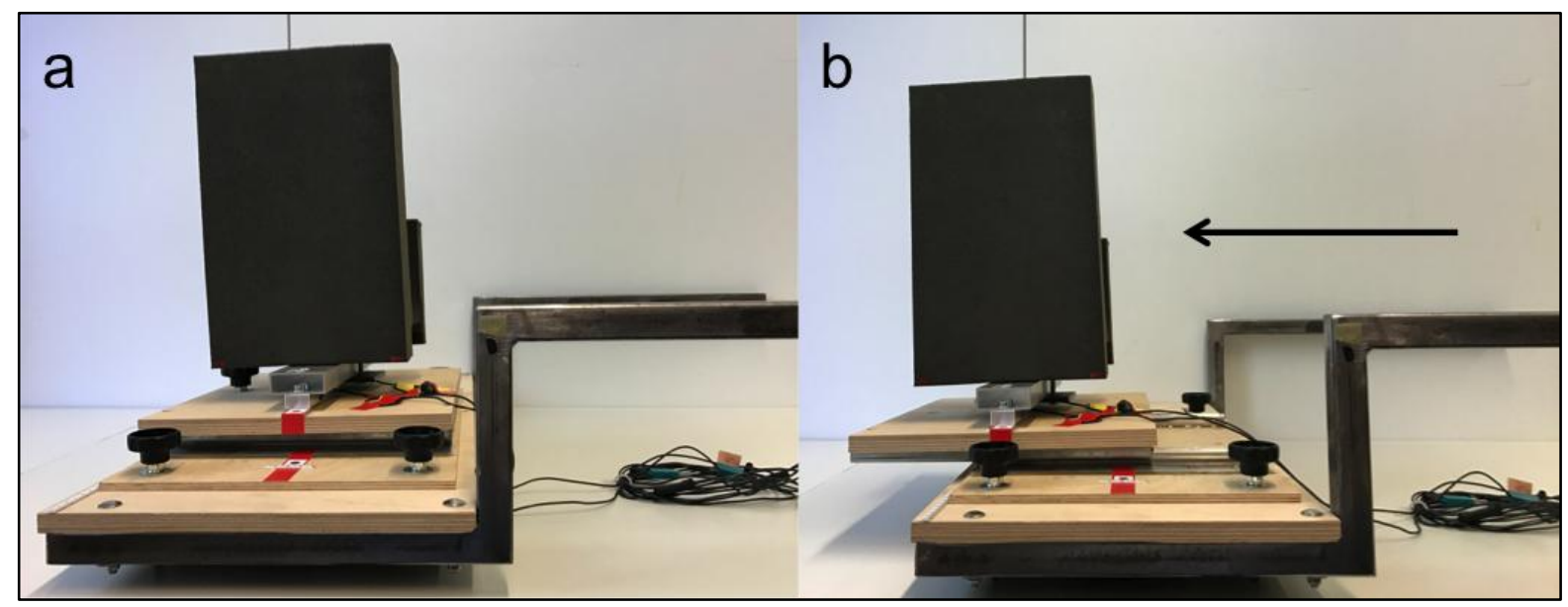

Abb. 23 Kraftmessplatte in Seitenansicht $(a=$ Kraftmessplatte in neutraler Position; $b=$ Kraftmessplatte in ausgefahrenem Zustand bei Ausgleich einer Longitudinalverschiebung) 
Die Kraftmessplatte besteht im Wesentlichen aus einer Grundplatte, die mit dem Hebelarm des ASLRT verbunden ist (Abb. 24). Die Grundplatte ist mit Langlöchern versehen, sodass die Kraftmessplatte seitlich verschiebbar und somit eine individuelle Positionierung des zu testenden Beines gewährleistet ist. Die Kraft wird über zwei seitlich angebrachte Biegebalken (Biovision, Wehrheim) gemessen, die zwischen einer Metallschiene und einem Vierkantrohr montiert sind (Abb. 24, d). Auf dem Vierkantrohr befindet sich eine U-förmige Fußablage, um das zu testende Bein dort abzulegen. Die U-förmige Fußablage ist an den Außenseiten erhöht, um eine Rotation des Beines während der Dehnung zu verhindern (Bohannon, Gajdosik, \& LeVeau, 1985). Die zwei Biegebalken (Kraftaufnehmer) registrieren die vertikal auftretende Gewichtskraft des Beines und die Kraft, die durch eine Dehnungsspannung an der ischiocruralen Muskulatur erzeugt wird. Aufgrund einer möglichen Rotationsbewegung des Beines kann es zu einer höheren Kraftübertragung auf der Außenseite der Kraftmessplatte kommen. Aus diesem Grund messen die Biegebalken zunächst unabhängig voneinander die auftretende Kraft. Aus diesen zwei Kraftwerten wird anschließend der Mittelwert errechnet, der dann zur Erfassung der Dehnungsspannung dient. 


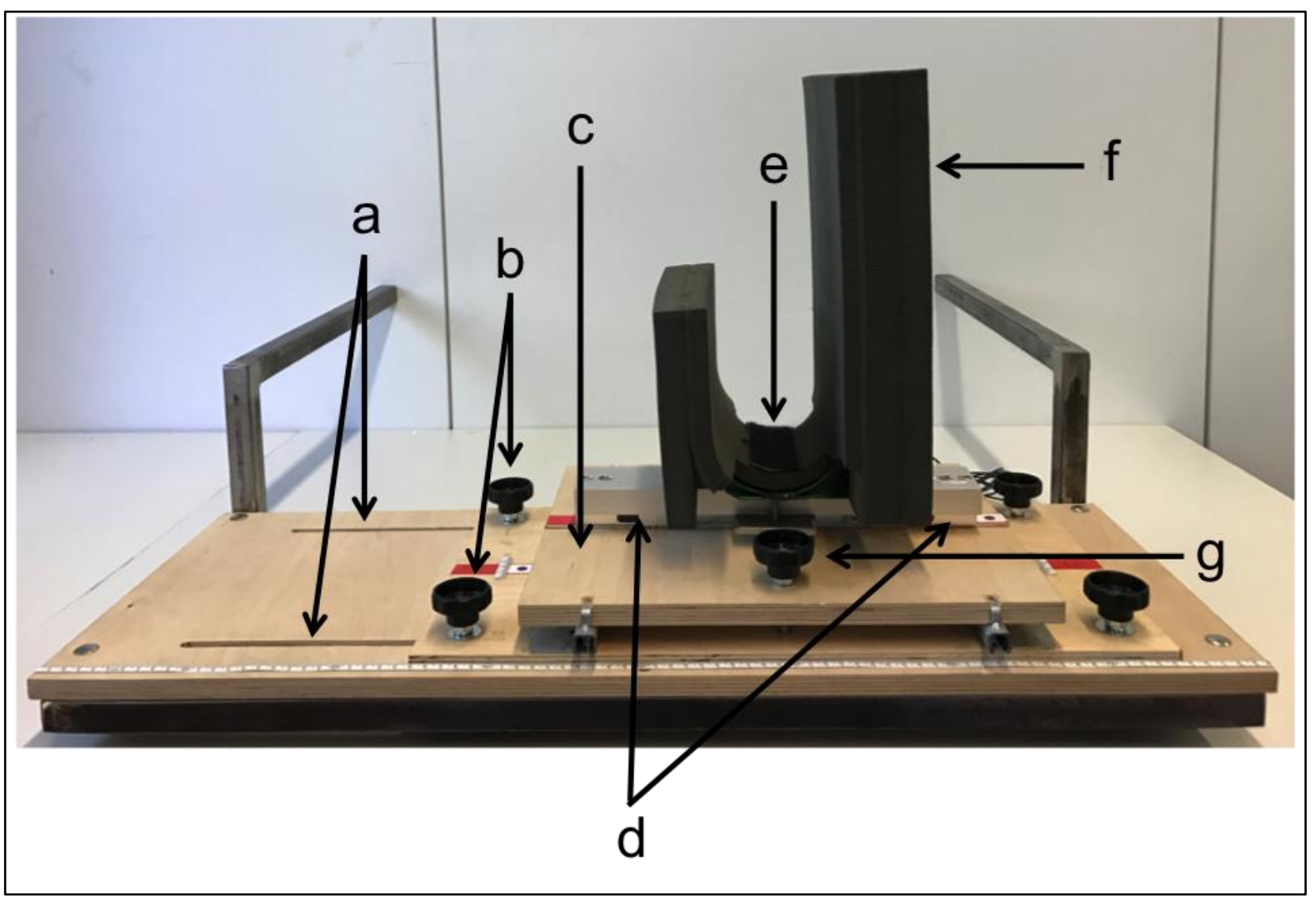

Abb. 24 Auf dem Hebelarm montierte Kraftmessplatte in Vorderansicht ( $a=$ Grundplatte mit Langlöchern zur seitlichen Verschiebung der Kraftmessplatte; $b=$ Schraube zur Fixierung der Kraftmessplatte in den Langlöchern; $c=$ Kraftmessplatte; $d=$ Biegebalken; $e=$ Klettband; $f=U$-förmige Fußablage; $g=$ Schraube zur Transportsicherung)

Um die Dehnungsspannung während einer Dehnungsprozedur zu erheben, wird der Fuß des zu testenden Beines in der U-förmigen Fußablage mittig abgelegt. Der Proband trägt hierfür eine mit Klettband versehene Sprunggelenksbandage (Mueller Sports Medicine Inc., Wisconsin) (Abb. 25). In der U-förmigen Fußablage befindet sich ebenfalls ein Klettband, um ein Verrutschen des Fußes innerhalb der Fußablage während des Dehnungsvorganges zu verhindern (Abb. 24, e). 


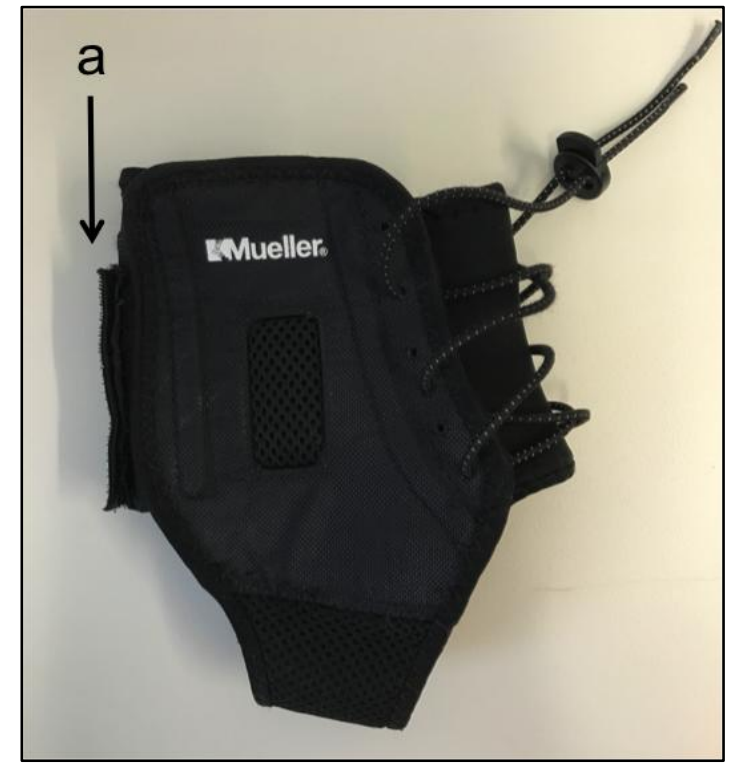

Abb. 25 Sprunggelenksbandage mit eingenähtem Klettband an der Rückseite ( $a=$ Klettband)

In einer Voruntersuchung ${ }^{8}$ wurden zwei Messverfahren zur Erhebung der Dehnungsspannung an der ischiocruralen Muskulatur verglichen (Haab et al., 2015). Hierzu wurden 17 Sportstudenten rekrutiert (Alter: $24.9 \pm 3.6$ Jahre, Größe: $174.6 \pm 8.5 \mathrm{~cm}$, Gewicht: $65.9 \pm 11 \mathrm{~kg}$ ), die nach einer Gewöhnungsphase einen Pre- und nach einer Woche einen Post-Test durchführten. Die Untersuchung wurde mit dem oben beschriebenen apparativen Straight-LegRaise-Test durchgeführt. Die Probanden absolvierten zu beiden Messzeitpunkten in randomisierter Reihenfolge die nachfolgenden zwei Messverfahren. Auf der einen Seite wurde das Verfahren nach Nahler (1998) angewendet, indem die Schritthöhe plus $8 \mathrm{~cm}$ als anatomische Drehachse ohne mögliche Longitudinalverschiebung herangezogen wurde. Auf der anderen Seite wurde die Dehnungsspannung mit der neu entwickelten Kraftmessplatte erhoben. Hier wurde der Trochanter major als anatomische Drehachse herangezogen mit der Möglichkeit, eine etwaige Longitudinalverschiebung des Beines auszugleichen. Gemessen wurde die Dehnungsspannung, berechnet als Drehmoment [Nm], mit beiden Messverfahren bei $5^{\circ}$ und $45^{\circ}$ Hüftflexionswinkel sowie am Punkt

${ }^{8}$ Die Untersuchung zur Evaluation der Kraftmessplatte wurde auf dem 22. Sportwissenschaftlichen Hochschultag der Deutschen Vereinigung für Sportwissenschaft im Oktober 2015 vorgestellt (Haab et al., 2015). 
der maximalen Beweglichkeit $\left(\mathrm{ROM}_{\text {max. }}\right)$. Diese drei Winkelmaße wurden deshalb gewählt, um zu untersuchen, inwieweit die Kraftmessplatte eine sehr geringe Dehnungsspannung, eine submaximale sowie eine maximale Dehnungsspannung erfasst. Untersucht wurde die Reliabilität und die Übereinstimmung beider Messverfahren mittels Konkordanz-Korrelations-Koeffizient $\left(r_{\mathrm{ccc}}\right)$ nach Lin (1992) und der Bland-Altman-Methode. Im Ergebnis konnte gezeigt werden, dass die Reliabilität der Dehnungsspannung bei $5^{\circ}, 45^{\circ}$ sowie bei der maximalen Beweglichkeit nach der Definition von Cohen (1988) als groß einzustufen ist (Tab. 13). Nach der Berechnung des Konkordanz-Korrelationskoeffizienten und der Bland-Altman-Methode stimmen beide Messverfahren hochgradig überein $\left(5^{\circ}=r_{c c c} \quad 0.92, \quad C l \quad\left[\begin{array}{lll}0.81 & 0.97\end{array}\right] ; 45^{\circ}=r_{c c c} \quad 0.95, \quad\left[\begin{array}{lll}C l & 0.87 & 0.98\end{array}\right]\right.$; $\left.R O M_{\text {max. }}=r_{c c c} 0.93, \mathrm{Cl}[0.860 .97]\right)$. Aufgrund dieser Ergebnisse stellt die neu entwickelte Kraftmessplatte ein geeignetes Messverfahren zur Messung der Dehnungsspannung dar.

Tab. 13 Reliabilitätskoeffizienten der Dehnungsspannung unterteilt in die beiden Messverfahren; $n=17$ ( $r=$ Pearson-Korrelationskoeffizient; $\left.{ }^{* *}=p<.01\right)$

\begin{tabular}{|c|c|c|}
\hline \multirow{2}{*}{ Winkelgrad } & \multicolumn{2}{|c|}{ Messverfahren } \\
\cline { 2 - 3 } & mit Longitudinalverschiebung & ohne Longitudinalverschiebung \\
\hline $5^{\circ}$ & $r=0.90^{* \star}$ & $r=0.93^{* *}$ \\
\hline $\mathbf{4 5}^{\circ}$ & $r=0.95^{* \star}$ & $r=0.87^{* *}$ \\
\hline ROM $_{\text {max. }}$ & $r=0.94^{* *}$ & $r=0.94^{* *}$ \\
\hline
\end{tabular}

\subsubsection{Abhängige Variablen}

Insgesamt lassen sich die abhängigen Variablen in biomechanische und neurophysiologische Parameter klassifizieren (Tab. 14), die nachfolgend erklärt werden. Anschließend wird konkret auf die Erhebung (Kap. 4.2.3) sowie die Auswertung (Kap. 4.2.4) der abhängigen Variablen eingegangen. 
Tab. 14 Beschreibung der abhängigen Variablen ( $a=$ biomechanische Parameter, $b=$ neurophysiologische Parameter; Erläuterungen siehe Text)

\begin{tabular}{|l|l|}
\hline \multicolumn{1}{|c|}{ Parameter } & \multicolumn{1}{c|}{ Beschreibung } \\
\hline Maximale Beweglichkeit $\left(\mathrm{ROM}_{\text {max. }}\right)^{\mathrm{a}}$ & Maximal zu erreichender Hüftflexionswinkel ( $\left.{ }^{\circ}\right)$ \\
\hline Maximale Dehnungsspannung $\left(\mathrm{Nm}_{\text {max. }}\right)^{\mathrm{a}}$ & $\begin{array}{l}\text { Maximal zu erreichende Dehnungsspannung beim Erreichen der } \\
\mathrm{ROM}_{\text {max. }}(\mathrm{Nm})\end{array}$ \\
\hline $\mathrm{PES}^{\mathrm{a}}$ & $\begin{array}{l}\text { Passive-elastische Stiffness bei einem Hüftflexionswinkel zwischen } \\
20^{\circ}-50^{\circ}\left(\mathrm{Nm} /{ }^{\circ}\right)\end{array}$ \\
\hline $\mathrm{PEE}^{\mathrm{a}}$ & $\begin{array}{l}\text { Passive-elastische Energie als Fläche unterhalb der Dehnungs- } \\
\text { spannungskurve }\left({ }^{\circ} \mathrm{Nm}\right)\end{array}$ \\
\hline $\mathrm{EMG}_{\text {onset }}^{\mathrm{b}}$ & Absoluter EMG-Anschaltzeitpunkt (Beginn einer Muskelaktivität) ( $\left.{ }^{\circ}\right)$ \\
\hline $\mathrm{EMG}_{\text {onset-\% }}{ }^{\mathrm{b}}$ & Relativer EMG-Anschaltzeitpunkt prozentual von ROM ${ }_{\text {max. }}(\%)$ \\
\hline
\end{tabular}

\subsection{Biomechanische Parameter}

Als primäre abhängige Variable zur Erfassung eines Effektes auf ein Dehntraining dient zumeist die maximale Beweglichkeit. Die Beweglichkeit ist zu einem großen Teil von der Muskeldehnfähigkeit abhängig (Alter, 2004; Klee \& Wiemann, 2005). Dabei scheinen es eher biomechanische Einflussfaktoren in Verbindung mit einer subjektiven Schmerztoleranzgrenze zu sein, die eine maximale Beweglichkeit limitieren (McHugh, Kremenic, Fox, \& Gleim, 1998; Weppler \& Magnusson, 2010). Die maximale Beweglichkeit $\left(\mathrm{ROM}_{\max }\right)$ beschreibt im Rahmen der hier vorliegenden Untersuchung die maximale Beweglichkeit beim Erreichen eines maximalen Hüftflexionswinkel in Rückenlage. Die maximale Dehnungsspannung ( $\left.\mathrm{Nm}_{\max }\right)$ ist definiert als mechanische Spannung der gedehnten Muskulatur in dem maximal zu erreichenden Gelenkwinkel, wobei das Erreichen der maximalen Beweglichkeit mit der maximal zu tolerierenden Dehnungsschmerzwahrnehmung einhergeht (Marshall \& Siegler, 2014).

Die Stiffness stellt die muskelphysiologische Response auf eine Muskeldehnung dar und wird auch als passive-elastische Eigenschaft der Muskulatur be- 
schrieben (Curwin, 2005; Gajdosik et al., 1999). Aus diesem Grund wird die Stiffness im Folgenden als passive-elastische Stiffness (PES) bezeichnet. Die PES errechnet sich aus der Dehnungsspannung sowie der Beweglichkeit und wurde in Anlehnung an Marshall, Cashman und Cheema (2011) zwischen einem Hüftflexionswinkel von $20^{\circ}$ und $50^{\circ}$ berechnet.

Eine weitere passive-elastische Eigenschaft der Muskulatur stellt die absorbierte Energie dar (Magnusson, 1998). Die absorbierte Energie während einer Muskeldehnung errechnet sich aus der Fläche unterhalb der Dehnungsspannungskurve und wird im Folgenden als passive-elastische Energie (PEE) bezeichnet. Die biomechanischen Parameter $\left(\mathrm{ROM}_{\max }, \mathrm{Nm}_{\max }, \mathrm{PES}, \mathrm{PEE}\right)$ sind in Abbildung 26 zusammengefasst.

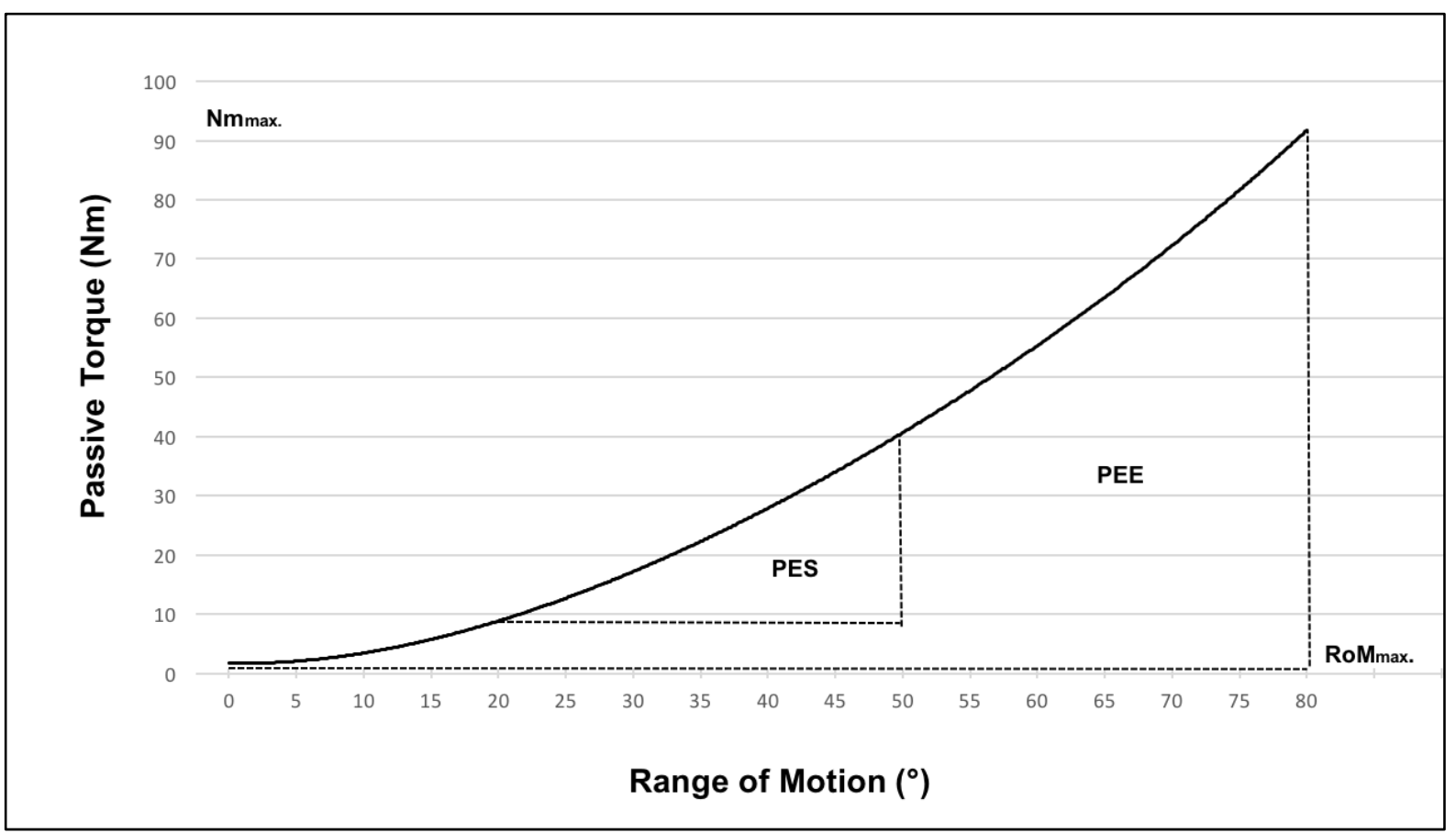

Abb. 26 Beispiel einer Dehnungsspannungskurve während einer Muskeldehnung mit Darstellung der abhängigen Variablen $\left(R O M_{\max }=\right.$ maximale Beweglichkeit, $\mathrm{Nm}_{\max }=$ maximale Dehnungsspannung, $P E S=$ passive-elastische Stiffness zwischen $20^{\circ}$ und $50^{\circ}, P E E=$ passive-elastische Energie als Fläche unterhalb der Kurve) (Haab \& Wydra, 2017, S. 1050) 


\subsection{Neurophysiologische Parameter}

Wird ein Muskel gedehnt, so kann es zu einer physiologischen Reflexantwort kommen, die elektromyographisch gemessen wird (vgl. S. 25). Weicht das elektromyographische Signal (EMG-Signal) während einer Muskeldehnung signifikant von seiner Baseline (Grundrauschen des EMG-Signals) ab, so wird dieser Zeitpunkt als EMG-Anschaltzeitpunkt beschrieben (Abb. 27), der im Folgenden als EMG-Onset bezeichnet wird (Cabido et al., 2016; Hodges \& Bui, 1996). Eine Muskeldehnung wird als passiv (ohne EMG-Onset) bezeichnet, wenn das EMG-Signal nicht signifikant von seiner Baseline abweicht.

In der Vergangenheit wurde berichtet, dass ein EMG-Onset kein limitierender Faktor für die Beweglichkeit darstellt (Magnusson et al., 1997, 1996; McHugh et al., 1998). In neueren Untersuchungen konnte allerdings gezeigt werden, dass es bei Personen mit geringerer Beweglichkeit zu einem früheren EMGOnset kommt als bei Personen mit größerer Beweglichkeit (Blazevich et al., 2012). Ebenso zeigten Ryan et al. (2014) in einem Vergleich zwischen jungen und alten Personen, dass es bei älteren Personen, mit einer geringeren Beweglichkeit, zu einem früheren EMG-Onset kommt als bei jüngeren Personen. Aus den vorangegangenen Überlegungen wurden neben den mechanischen Einflussfaktoren auf die Beweglichkeit ebenso das EMG-Onset als zusätzlicher neurophysiologischer Parameter als abhängige Variable definiert ${ }^{9}$. Der EMGOnset wurde in der hier vorliegenden Untersuchung definiert als EMG-Aktivität, die, in Anlehnung an Blazevich et al. (2012) sowie Hodges und Bui (1996), für $100 \mathrm{~ms}$ drei Standardabweichungen von der Baseline abweicht. Es wurde der absolute EMG-Onset und der relative EMG-Onset erhoben. Der absolute EMGOnset (EMG onset $_{\text {) }}$ wurde definiert als erste signifikante Abweichung des EMG-

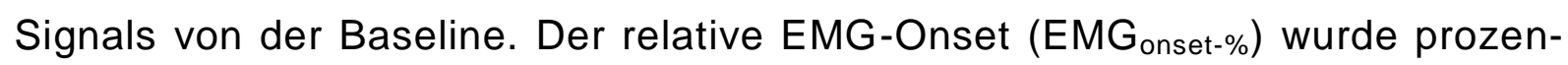
tual von der maximal erreichten Beweglichkeit $\left(R_{0} M_{\text {max. }}\right)$ errechnet (Abb. 27).

9 Die Untersuchung speziell zu den dargestellten EMG-Parametern wurde unter dem Titel „EMG onset after a 10 week stretching intervention. A comparison between older and younger adults" in der Zeitschrift Journal of Exercise Physiology Online im August 2017 veröffentlicht (Haab, Massing, \& Wydra, 2017). 


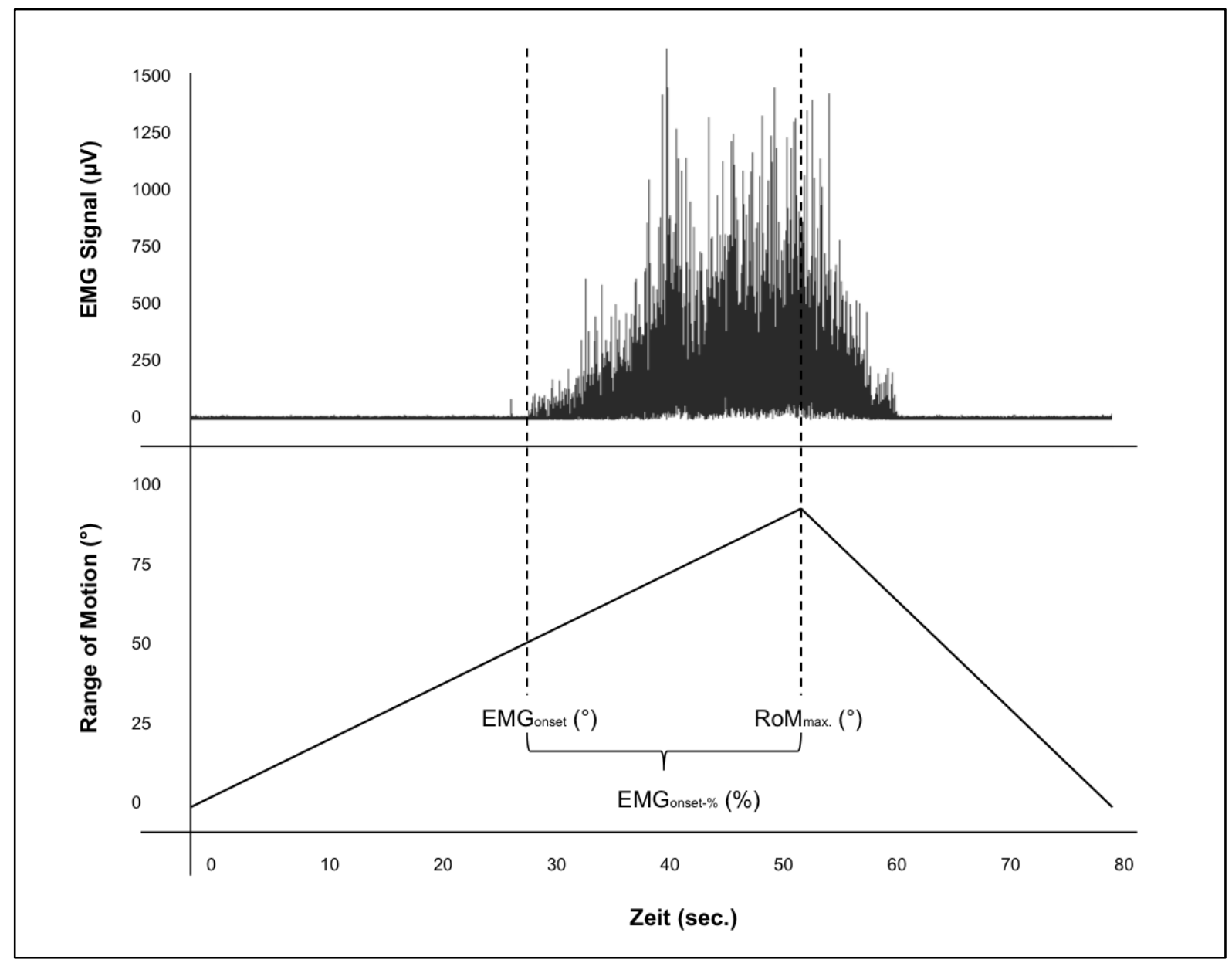

Abb. 27 Beispielhafte Darstellung der EMG-Parameter eines Probanden $\left(R O M_{\max }=\right.$ maximale Beweglichkeit, $E M G_{\text {onset }}=$ Gelenkwinkel beim $E M G$-Onset; $E M G_{\text {onset }-\%}=$ EMG-Onset prozentual von ROM max.) (mod. nach Haab, Massing, \& Wydra, 2017, S. 125)

\subsubsection{Schmerzskala}

Neben den oben beschriebenen abhängigen Variablen (biomechanische und neurophysiologische Parameter) wurde weiterhin das Schmerzempfinden während der maximalen Muskeldehnung erhoben. Wie bereits erwähnt, ist das Erreichen einer maximalen Beweglichkeit stark vom individuellen subjektiven Schmerzempfinden abhängig. Der Dehnungsschmerz wurde über eine $100 \mathrm{~mm}$ Schmerzskala objektiviert (Hawker, Mian, Kendzerska, \& French, 2011). Die Schmerzskala wurde den Probanden direkt nach dem Herunterfahren des $\mathrm{He}$ belarmes, also unmittelbar nach Beendigung der Dehnungsprozedur auf dem ASLRT, gezeigt. Auf einer horizontal verlaufenden $100 \mathrm{~mm}$ langen Linie sollten 
die Probanden ihr Schmerzempfinden bei Erreichen der maximalen Beweglichkeit mit einem vertikalen Strich markieren $(0 \mathrm{~mm}=$ kein Dehnungsschmerz; $100 \mathrm{~mm}=$ maximal zu ertragender Dehnungsschmerz).

\subsubsection{Reliabilität der abhängigen Variablen}

Die Reliabilität der abhängigen Variablen wurde für beide Altersgruppen mit einem Pearson-Korrelationskoeffizienten berechnet. Hierfür wurden in Vortests während der Gewöhnungsphase (vgl. Abb. 34) an zwei separaten Terminen mit einer Woche Abstand die Daten erhoben. In Anlehnung an Cohen (1988) wird die Reliabilität aller abhängigen Variablen als groß bewertet (Tab. 15). Anzumerken ist hier, dass während den Vortests drei ältere und drei jüngere Probanden bei mindestens einem Messzeitpunkt kein EMG-Onset zeigten.

Tab. 15 Reliabilität der abhängigen Variablen $\left(R O M_{\max }=\right.$ maximale Beweglichkeit, $\mathrm{Nm}_{\max }=$ maximale Dehnungsspannung, $P E S=$ passive-elastische Stiffness, $P E E=$ passive-elastische Energie, $E M G_{\text {onset }}=$ Gelenkwinkel beim EMG-Onset; $E M G_{\text {onset } \%}=E M G$-Onset prozentual von $R O M_{\text {max. }}$.

\begin{tabular}{|c|c|c|c|c|}
\hline Abhängige Variablen & Altersgruppe & $\mathbf{n}$ & Korrelationskoeffizient $(r)$ & Signifikanzniveau $(p)$ \\
\hline \multirow{2}{*}{$\mathrm{ROM}_{\max .}\left(^{\circ}\right)$} & jung & 15 & 0.95 & $<.001$ \\
\hline & alt & 14 & 0.83 & $<.001$ \\
\hline \multirow{2}{*}{$\mathrm{Nm}_{\max .}(\mathrm{Nm})$} & jung & 15 & 0.97 & $<.001$ \\
\hline & alt & 14 & 0.84 & $<.01$ \\
\hline \multirow{2}{*}{$\operatorname{PES}\left(\mathrm{Nm} /{ }^{\circ}\right)$} & jung & 15 & 0.88 & $<.001$ \\
\hline & alt & 14 & 0.91 & $<.001$ \\
\hline \multirow{2}{*}{ PEE $\left({ }^{\circ} \mathrm{Nm}\right)$} & jung & 15 & 0.96 & $<.001$ \\
\hline & alt & 14 & 0.89 & $<.001$ \\
\hline \multirow{2}{*}{$\mathrm{EMG}_{\text {onset }}\left({ }^{\circ}\right)$} & jung & 12 & 0.88 & $<.001$ \\
\hline & alt & 11 & 0.77 & $<.05$ \\
\hline \multirow{2}{*}{$\mathrm{EMG}_{\text {onset-\% }}(\%)$} & jung & 12 & 0.79 & $<.001$ \\
\hline & alt & 11 & 0.73 & $<.05$ \\
\hline \multirow{2}{*}{ Schmerzskala $(\mathrm{mm})$} & jung & 15 & 0.74 & $<.01$ \\
\hline & alt & 14 & 0.85 & $<.001$ \\
\hline
\end{tabular}




\subsubsection{Datenerhebung}

Die zeitsynchrone Datenerhebung während der Dehnungsprozedur auf dem ASLRT erfolgte am linken Bein mit der Software DasyLab (National Instruments Ireland Resources Limited, Version 10) über einen 16-Kanal A/D-Wandler (Biovision, Wehrheim) mit einer Frequenz von $1000 \mathrm{~Hz}$. Der A/D-Wandler war per USB-Kabel mit einem Laptop (Toshiba, Satellite Pro L650-156) verbunden. Das Schaltbild zur Erhebung der abhängigen Variablen ist in Abbildung 28 dargestellt. Um sicherzustellen, dass die Datenerhebung nicht durch ein unwillkürliches Beugen des zu testenden Beines verfälscht wurde, wurde der Dehnungsvorgang zusätzlich mit einer Videokamera (Sony, HDR-PJ530E) aufgezeichnet.

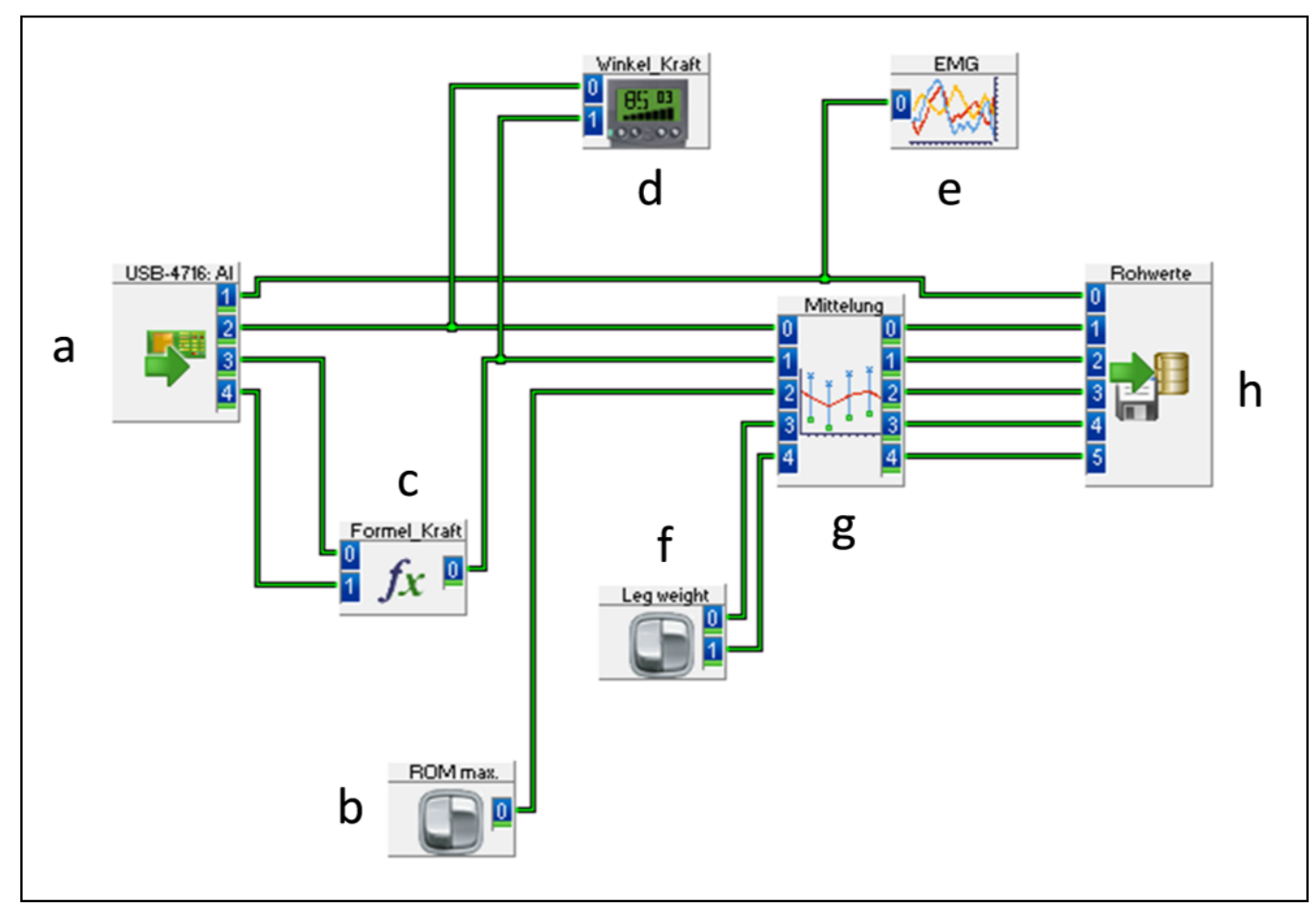

Abb. 28 Schaltbild aus DasyLab zur Datenerhebung (a = USB-Eingang zum Laptop vom $A / D$-Wandler (Kanal $1=$ EMG-Ableitung, Kanal $2=$ Drehpotentiometer, Kanal 3 \& $4=$ Kraftmessplatte), $b=$ Taste (Trigger) zur Aufnahme der maximalen Beweglichkeit, $c=$ Zusammenführen der Kraftwerte aus den zwei Biegebalken der Kraftmessplatte, $d$ = Visualisierung der Kraft (Dehnungsspannung) und der Gelenkwinkel (Beweglichkeit), $e=$ Visualisierung des EMG-Signals, $f=$ Schalter zur Aufnahme der Beingewichtskraft, $g=$ Mittelung der Aufnahmefrequenz der Kraft- und Winkelparameter, $h=$ Speicherung der Rohdaten) 
Die Datenerhebung mit DasyLab erfolgte in zwei Schritten. In einem dritten Schritt wurden anschließend die erhobenen Daten mit MatLab (MathWorks Inc., Version R2014a) ausgewertet (Kapitel 4.2.4).

Sämtliche erhobenen Daten wurden zunächst in einem ersten Schritt als Rohdaten in einem DasyLab-Format (ddf) abgespeichert. Hierbei wurden die Winkel- und Kraftparameter zur Datenreduktion blockweise gemittelt (Abb. 28, g), sodass diese Rohdaten letztlich mit einer Frequenz von $500 \mathrm{~Hz}$ abgespeichert wurden. Das EMG-Signal wurde mit einer Frequenz von $1000 \mathrm{~Hz}$ abgespeichert. Aus den im ersten Schritt abgespeicherten Rohdaten Beweglichkeit [ ${ }^{\circ}$ ], Dehnungsspannung [N] sowie das Roh-EMG-Signal $[\mu \mathrm{V}]$ wurden anschließend die abhängigen Variablen abgeleitet.

Nach der Datenerhebung wurden die Rohdaten in einem zweiten Schritt in DasyLab weiterverarbeitet (Abb. 29). Hierfür wurden die Rohdaten aus der Datenerhebung zunächst eingelesen (Abb. 29, a). Anschließend wurde die gemessene Dehnungsspannung $[\mathrm{N}]$ in ein individuelles Drehmoment $[\mathrm{Nm}]$ umgerechnet und die Dehnungsspannungskurve mit einem Polynom 4. Ordnung geglättet (Abb. 29, c) sowie das Roh-EMG-Signal gefiltert (Abb. 29, b). Die einzelnen Bearbeitungsschritte der Rohdaten werden in den nachfolgenden Kapiteln, getrennt nach Winkel-, Kraft und EMG-Parameter, ausführlich dargestellt. Die im zweiten Schritt weiterverarbeiteten Daten wurden als ASCII-Format abgespeichert, um diese anschließend mit MatLab auswerten zu können (Kapitel 4.2.4). 


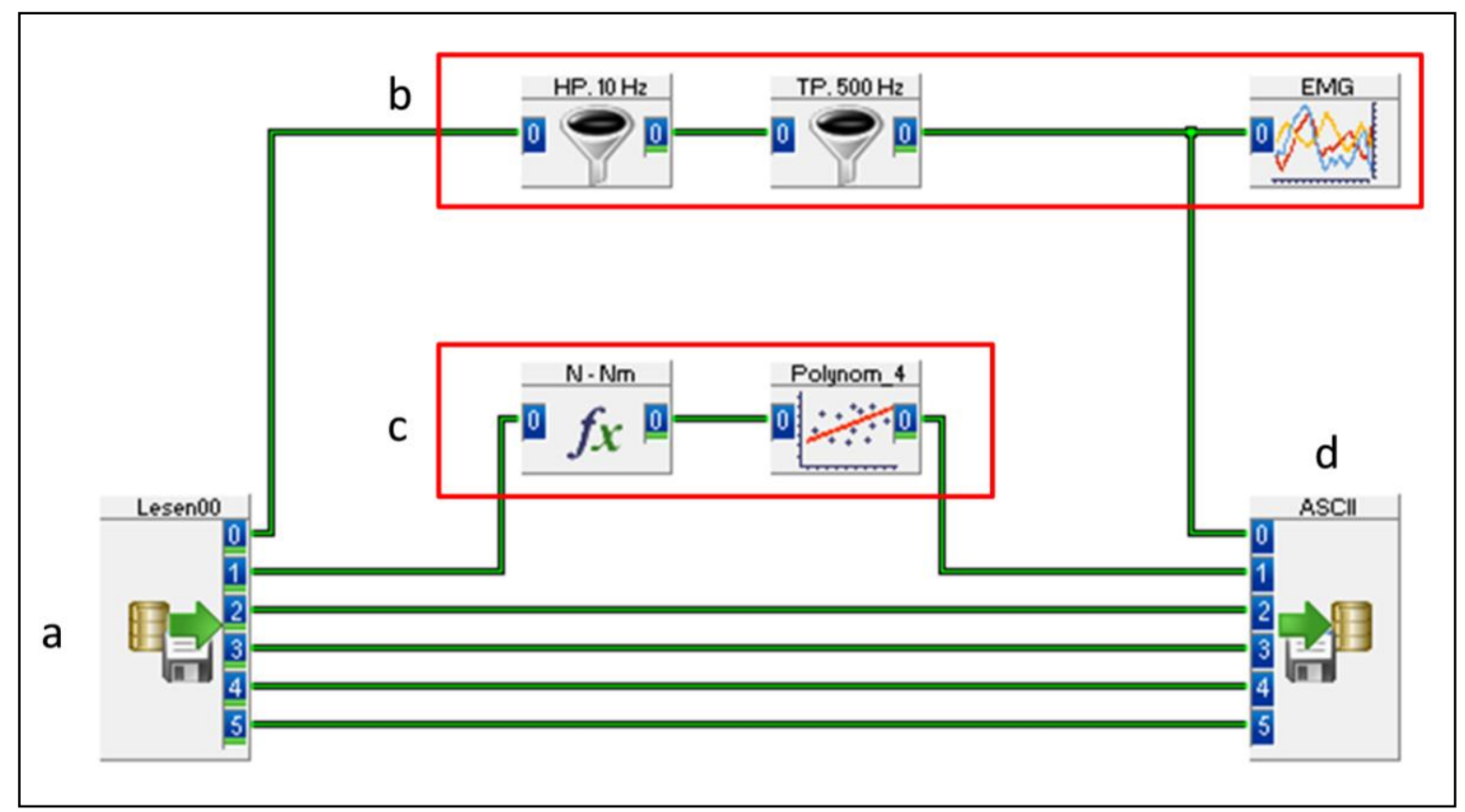

Abb. 29 Schaltbild aus DasyLab zur Weiterverarbeitung der Rohdaten aus der Datenerhebung $(a=$ einlesen der Rohdaten, $b=$ Filterung des EMG-Signals und nochmalige visuelle Überprüfung, $c=$ Umrechnung der Dehnungsspannung von Kraft [N] in ein individuelles Drehmoment [Nm] sowie Glättung der Dehnungsspannungskurve, $d=$ Speicherung der Daten als ASCII-Format)

In den nachfolgenden Kapiteln wird die Erhebung der abhängigen Variablen mit DasyLab und anschließend die Auswertung der Daten mit MatLab ausführlich beschrieben. Die abhängigen Variablen werden hierbei als Winkelparameter $\left(R_{\text {max. }}\right)$, Kraftparameter ( $\mathrm{Nm}_{\text {max. }}$, PES, PEE) und EMG-Parameter (EMG onset, $E M_{\text {onset-\%) }}$ zusammengefasst.

\subsubsection{Erhebung der Winkelparameter}

Die Beweglichkeit wurde durch einen Drehpotentiometer (Biovision, Wehrheim) gemessen, der sich in der Drehachse des ASLRT befand (Abb. 21, a). So war es möglich, die Winkelwerte der Hüftflexion aufzunehmen. Der Proband wurde auf dem ASLRT so positioniert, dass der Trochanter major mit der Drehachse des ASLRT übereinstimmte. An der Drehachse des ASLRT war ein Laserpointer verbaut, mit dem der Trochanter major angepeilt wurde. 
Die Daten der maximalen Beweglichkeit ( $\left.\mathrm{ROM}_{\text {max. }}\right)$ wurden aufgenommen, indem über das DasyLab-Schaltbild eine Taste betätigt und damit der Punkt der maximalen Beweglichkeit markiert wurde (Abb. 30, b). Dieser Trigger wurde dann später in der Weiterverarbeitung der Rohdaten in der ASCII-Datei sichtbar.

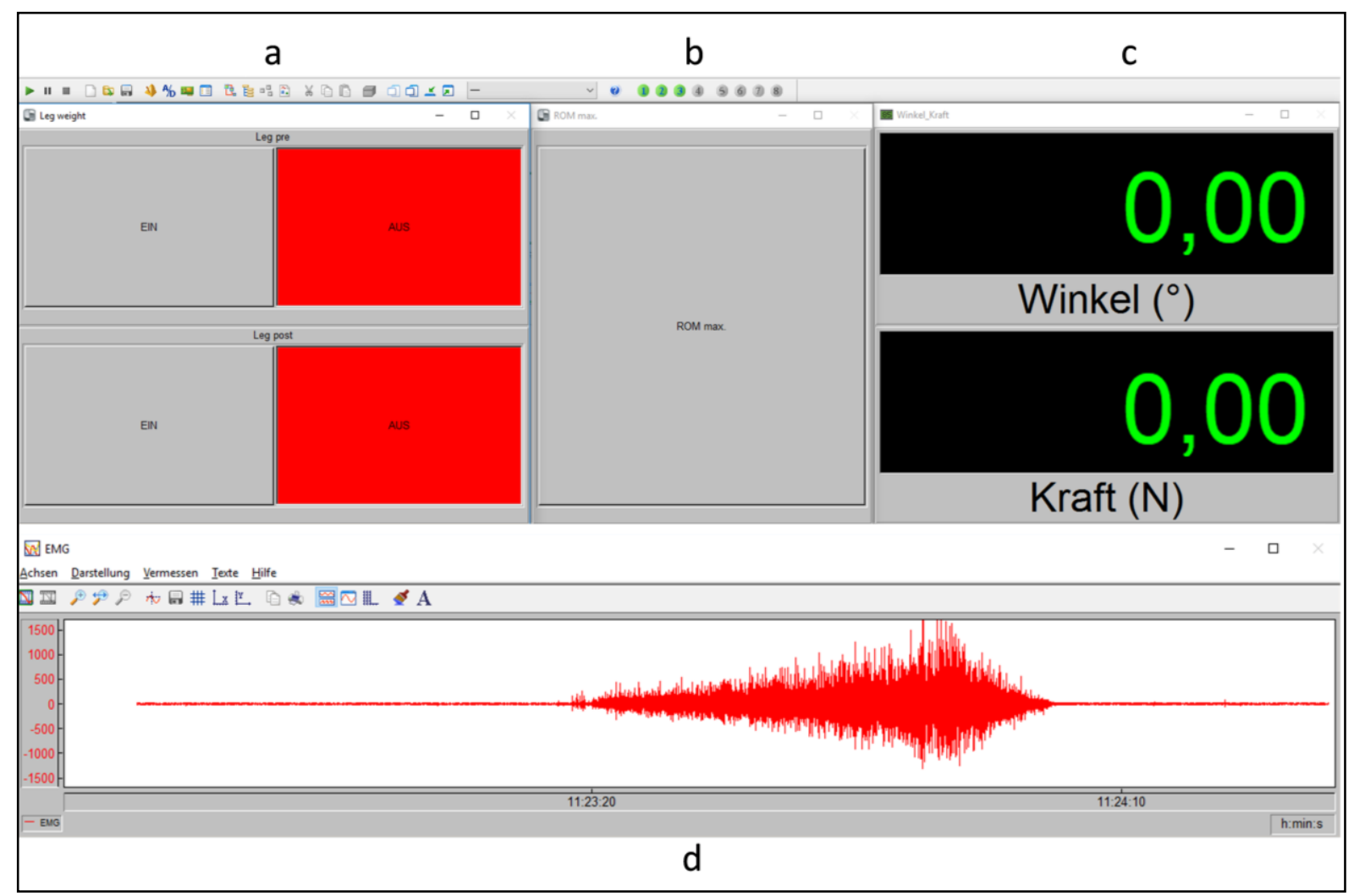

Abb. 30 Steuerung der Datenerhebung in DasyLab (siehe Module aus dem Schaltbild in Abb. 28) (a = Schalter zur Aufnahme der Beingewichtskraft vor und nach einem Dehnungsvorgang, vgl. Abb. 28, $f ; b=$ Taste (Trigger) zur Aufnahme der maximalen Beweglichkeit, vgl. Abb. 28, b; $c=$ Visualisierung der Kraft (Dehnungsspannung) und der Gelenkwinkel (Beweglichkeit), vgl. Abb. 28, $d ; d=$ Visualisierung des EMG-Signals; vgl. Abb. 28, e)

\subsubsection{Erhebung der Kraftparameter}

Die Datenaufnahme der Kraftparameter erfolgte über die Kraftmessplatte und wurde zunächst in Newton [N] gemessen und als Rohwert abgespeichert (Abb. 28, h). Um die Dehnungsspannung zwischen den Probanden vergleichbar zu machen, wurde diese in einem zweiten Schritt an der individuellen Bein- 
länge der Probanden relativiert (Abb. 29, c). Hierfür wurde zu Testbeginn die Beinlänge von Fußboden bis Trochanter major gemessen. Im Nachhinein wurde aus der Multiplikation der Beinlänge und der gemessenen Kraft das Drehmoment $[\mathrm{Nm}]$ als Parameter der Dehnungsspannung berechnet. Die Berechnung der Dehnungsspannung erfolgte bereinigt durch das Beingewicht und der Schwerkraft (vgl. Kapitel 4.2.4.2). Das Beingewicht wurde erfasst, indem der Proband entspannt auf dem ASLRT für fünf Sekunden in horizontaler Lage liegen blieb. Hierdurch wurde die Beingewichtskraft in Anlehnung an Warren, Hardiman und Wolf (1992) gemessen. Im Anschluss an den Dehnungsvorgang, nachdem der Hebelarm wieder in der horizontalen Ausgangslage angekommen war, wurde erneut die Beingewichtskraft gemessen. Diese zweite Messung ist notwendig, da es aufgrund von Flüssigkeitsverschiebungen im Bein, durch den Dehnungsvorgang auf dem ASLRT, zu einer Veränderung der Beingewichtskraft kommt (Klee, 2003; Klee \& Wiemann, 2000). Die Aufzeichnung der Beingewichtskraft erfolgte durch einen Schalter in DasyLab (Abb. 30, a). Zur Glättung der aufgezeichneten Dehnungsspannungskurve wurde ein Polynom 4. Ordnung herangezogen (Abb. 29, c).

Die maximale Dehnungsspannung $\left(\mathrm{Nm}_{\text {max. }}\right)$ wurde aufgenommen, indem über das DasyLab-Schaltbild eine Taste beim Erreichen der maximalen Beweglichkeit betätigt und so der Punkt der maximalen Dehnungsspannung zeitgleich mit der maximalen Beweglichkeit markiert wurde (Abb. 30, b). Dieser Trigger wurde dann später in der Weiterverarbeitung der Rohdaten in der ASCII-Datei sichtbar. Die weiteren Kraftparameter (PES, PEE) wurden anschließend mit MatLab berechnet (Kapitel 4.2.4.2).

\subsubsection{Erhebung der EMG-Parameter}

Bevor eine adäquate Erfassung des EMG-Signals während der Datenaufnahme durchgeführt werden konnte, wurden vorab sämtliche Störungsquellen eruiert. Hierzu wurden in Vortests mittels Frequenzanalysen die unterschiedlichen Störungsquellen auf die Elektromyographie identifiziert. Dazu zählten insbesondere der Elektromotor des ASLRT sowie ein Metallschränkchen, auf dem der Lap- 
top zur Datenaufnahme stand. Weitere gängige Störungsquellen, wie beispielsweise mobile Endgeräte (Handy, Tablet, etc.), wurden während der Datenaufnahme ausgeschaltet. Bei eingeschaltetem Elektromotor wurde das EMG-Signal gestört. Dies war allerdings nur dann der Fall, wenn der Laptop auf dem vorgesehenen Metallschränkchen stand, da es als eine Art Antenne fungierte. Nachdem zwischen Laptop und Metallschränkchen eine Plastikbox gestellt wurde, konnte das Störsignal beseitigt werden. Des Weiteren wurde ein abgeschirmtes USB-Kabel (Dr. Zellmer GmbH, Sankt Augustin) zur Verbindung des A/D-Wandlers mit dem Laptop verwendet. Außerdem musste während der Datenaufnahme das Netzkabel vom Laptop entfernt werden, da es sonst zu einem typischen $50 \mathrm{~Hz}-N e t z b r u m m e n$ (Rauschen) im EMG-Signal kam. Letztendlich konnten sämtliche Störungen im EMG-Signal eliminiert werden.

Während des Dehnungsvorganges mit dem ASLRT wurde die Muskelaktivität per Oberflächen-Elektromyographie (Biovision, Wehrheim) gemessen, um auf der einen Seite sicherzustellen, dass während der Testung die Muskulatur nicht aktiv war, und um auf der anderen Seite eine mögliche Veränderung der Muskelaktivität zu überprüfen. Während der Datenerhebung wurden die EMGSignale um das 5000-fache verstärkt. Die EMG-Elektroden wurden in Anlehnung an De Luca (1997) und Hermens et al. (1999) appliziert. Zunächst wurden die Haare an dem betreffenden Hautareal mit einem Einwegrasierer entfernt. Danach wurde das rasierte Areal mit abrasiver Paste (Everi, Spes Medica) abgerieben und abschließend mit einem alkoholischen Hautantiseptikum (Kodan Tinktur Forte, Schülke \& Mayr) gereinigt. Die EMG-Ableitungen erfolgten auf dem Muskelbauch in Faserlängsrichtung des langen Kopfes von M. biceps femoris mit zwei Einwegelektroden (Ambu, Blue Sensor N) mit einem Interelektrodenabstand von $20 \mathrm{~mm}$ (Abb. 31). Der M. biceps femoris wurde als Repräsentant der ischiocruralen Muskulatur gewählt, da Schönthaler und Ohlendorf (2002, S. 125) zeigen konnten, dass die Mm. semimembranosus und semitendinosus nicht so gut zur Ableitung der Muskelaktivität während einer Dehnung an einem ASLRT geeignet sind. Eine weitere Einwegelektrode wurde als Referenzelektrode an der Schienbeinkante angebracht. 


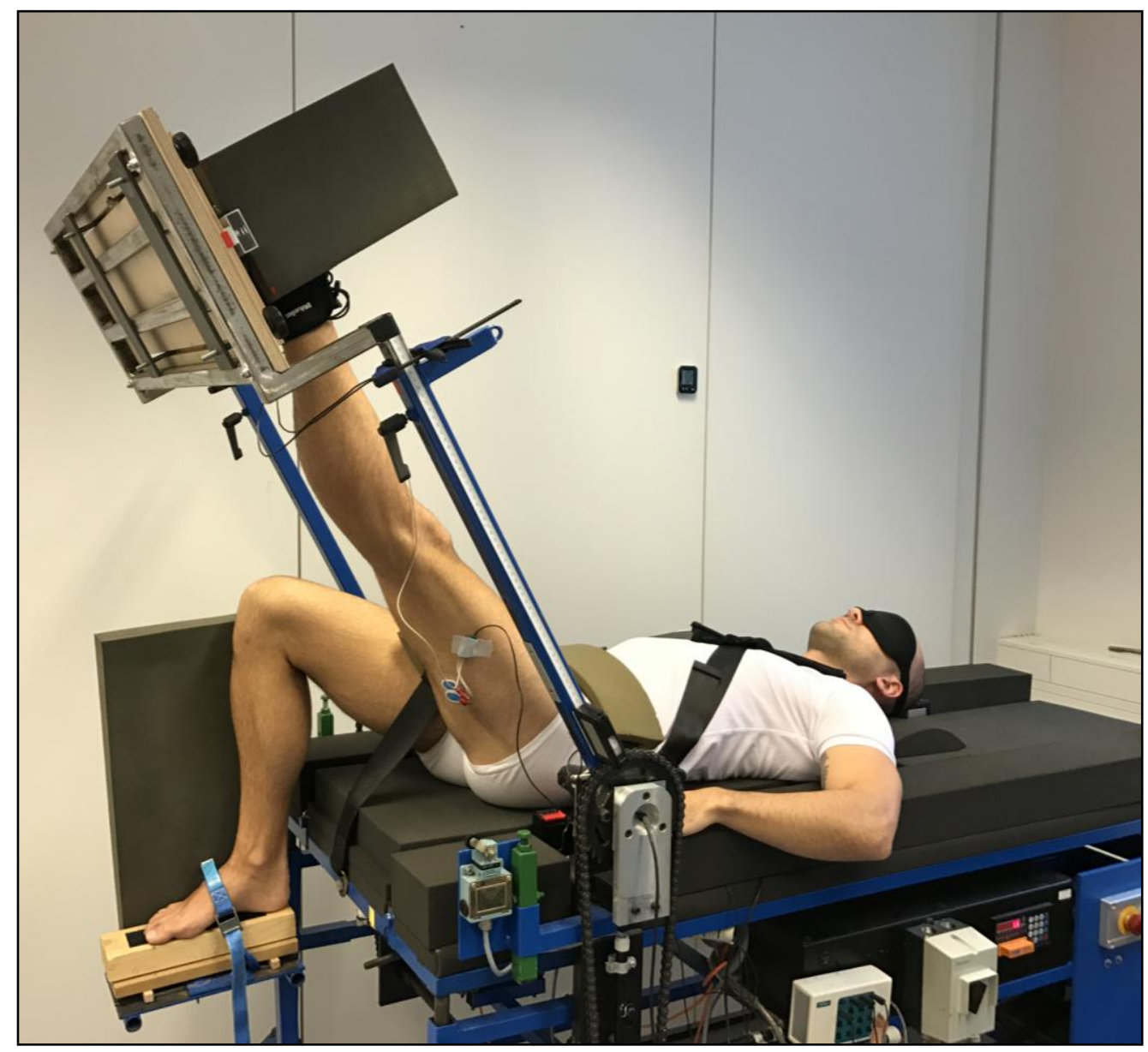

Abb. 31 Proband auf ASLRT mit EMG-Ableitungen an M. biceps femoris (Haab, Massing, \& Wydra , 2017, S. 123)

Die elektromyographischen Signale wurden während der Dehnungsprozedur durchgehend aufgezeichnet und als Rohdaten abgespeichert. Anschließend wurden die EMG-Signale über einen $10 \mathrm{~Hz}$-Hochpass- und einen $500 \mathrm{~Hz}$ Tiefpassfilter sowie einem Butterworth-Filter 4. Ordnung gefiltert und als ASCIIDatei abgespeichert (Abb. 29, b). Die Weiterverarbeitung der gefilterten EMGSignale zur Berechnung des EMG-Onset erfolgte dann mit MatLab (Kapitel 4.2.4.3).

\subsubsection{Datenauswertung}

Zunächst wurde mit dem aufgenommenen Video visuell überprüft, ob das zu testende Bein während des Dehnungsvorganges gebeugt wurde. Dies war in 
keiner Aufnahme der Fall, sodass anschließend die Auswertung der aufgezeichneten Messdaten erfolgte.

Die mit DasyLab aufgenommenen Parameter wurden zur Auswertung zunächst aus dem ASCII-Format in ein Microsoft-Excel-Format (xIsx) übertragen und anschließend in MatLab (MathWorks Inc., Version R2014a) importiert. In den nachfolgenden Kapiteln wird die Weiterverarbeitung der Winkel-, Kraft- und EMG-Parameter ausführlich erläutert. Aus den durch MatLab ausgewerteten Daten ergaben sich die abhängigen Variablen, die letztlich für die abschließenden statistischen Analysen mit SPSS (IBM Corp., Version 23) herangezogen wurden.

\subsubsection{Auswertung der Winkelparameter}

Während der Datenerhebung wurde das Erreichen der maximalen Beweglichkeit $\left(R_{\text {OMax. }}\right)$ mit DasyLab getriggert (Abb. 28, b). Aus den Messdaten des gesamten Dehnungsvorgangs wurden die aufgenommenen Winkelwerte zwischen der Ausgangsposition (Hüfte in Null-Grad-Stellung) bis zu dem getriggerten Signal (Bein in maximaler Hüftflexionsstellung) herausgefiltert. Zur Darstellung der $\mathrm{ROM}_{\max }$. wurde anschließend der Mittelwert der letzten $500 \mathrm{Da}$ tenpunkte errechnet. Die maximale Bewegungsreichweite wurde in MatLab als maximaler Winkelwert $\left[^{\circ}\right]$ berechnet und abschließend zur statistischen Weiterverarbeitung in SPSS übertragen. Der MatLab-Code zur Berechnung der maximalen Beweglichkeit ist im Anhang dargestellt (S. 180).

\subsubsection{Auswertung der Kraftparameter}

$\mathrm{Zu}$ den Kraftparametern gehören die maximale Dehnungsspannung ( $\left.\mathrm{Nm}_{\max }\right)$, die passive-elastische Stiffness (PES) sowie die passive-elastische Energie (PEE) (Abb. 26). Die erhobenen Kraftparameter wurden um das Beingewicht und die Schwerkraft bereinigt. Die Beingewichtskraft wurde vor jedem Dehnungsvorgang innerhalb von fünf Sekunden gemessen. Die Bereinigung der Kraftparameter durch die Beingewichtskraft und die Schwerkraft erfolgte über eine trigonometrische Kosinusfunktion. Des Weiteren mussten die Kraftwerte 
noch zusätzlich um das Gewicht der Kraftmessplatte bereinigt werden. Hierzu wurde das Eigengewicht der am Hebelarm montierten Kraftmessplatte ohne Proband über den gesamten Bewegungsradius eines Dehnungsvorganges gemessen und als Referenzmessung mit Kraft- und Winkelwerten gespeichert. Diese Referenzmessung wurde sodann in Beziehung zu den Messdaten mit den Probanden gesetzt, indem der Koeffizient eines Interpolationspolynoms zwischen der Referenzmessung ohne Proband und der „realen“ Messung mit Proband errechnet wurde (vgl. MatLab-Code zur Berechnung der maximalen Dehnungsspannung, S. 181).

\subsection{Maximale Dehnungsspannung}

Die maximale Dehnungsspannung wurde in MatLab als maximales Drehmoment $[\mathrm{Nm}]$ berechnet. Der MatLab-Code zur Berechnung der maximalen Dehnungsspannung ist im Anhang dargestellt (S. 181).

\subsection{Passive-elastische Stiffness}

Die Auswertung der passiven-elastischen Stiffness (PES) erfolgte, indem zunächst die durch die Beingewichtskraft, die Schwerkraft und die Gewichtskraft der Kraftmessplatte bereinigten Kraftparameter in den MatLab-Code importiert wurden. Die PES wurde zwischen $20^{\circ}$ - und $50^{\circ}$-Hüftflexionswinkel erhoben. Hierfür wurde die mittlere Dehnungsspannung bei einem Hüftflexionswinkel von $20^{\circ}$ und $50^{\circ}$ ermittelt. Um die Kraftwerte für den Hüftflexionswinkel von $20^{\circ} \mathrm{zu}$ erhalten, wurde demnach die mittlere Dehnungsspannung zwischen $19.5^{\circ}$ und $20.5^{\circ}$ berechnet. Für den Hüftflexionswinkel von $50^{\circ}$ wurde die mittlere Dehnungsspannung zwischen $49.5^{\circ}$ und $50.5^{\circ}$ berechnet. Die passive-elastische Stiffness wurde in MatLab als Differenz der Dehnungsspannungswerte $(\Delta \mathrm{Nm})$ dividiert durch die Differenz der Winkelwerte $\left(\Delta^{\circ}\right)$ zwischen $20^{\circ}$ - und $50^{\circ}$ Hüftflexion $\left[\mathrm{Nm} /{ }^{\circ}\right]$ berechnet. Der MatLab-Code zur Berechnung der passivenelastischen Stiffness ist im Anhang dargestellt (S. 182). 


\subsection{Passive-elastische Energie}

Zur Auswertung der passiven-elastischen Energie wurden zunächst wie im vorherigen Kapitel beschrieben die bereinigten Kraftparameter in den MatLabCode importiert. Anschließend wurde die Fläche unterhalb der Dehnungsspannungskurve mit einer Trapezfunktion errechnet. Die passive-elastische Energie wurde in MatLab als Integral der Dehnungsspannungskurve [ $\left.{ }^{\circ} \mathrm{Nm}\right]$ berechnet. Der MatLab-Code zur Berechnung der passiven-elastischen Energie ist im Anhang dargestellt (S. 183).

\subsubsection{Auswertung der EMG-Parameter}

Die Auswertung der EMG-Parameter beinhaltet die Darstellung des absoluten EMG-Onset ( $E_{M G_{\text {onset }}}$ ) sowie das relativ zur maximalen Beweglichkeit errechnete EMG-Onset $\left(\mathrm{EMG}_{\text {onset-\%) }}\right.$. Der MatLab-Code zur Darstellung des EMGOnset wurde in modifizierter Form von Hodges und Bui (1996) übernommen. In der hier vorliegenden Arbeit wurde das EMG-Onset in Anlehnung an Blazevich et al. (2012) sowie Hodges und Bui (1996) definiert als eine Muskelaktivität, die für $100 \mathrm{~ms}$ drei Standardabweichungen von der Baseline abweicht. Zur Berechnung des EMG-Onset wurde das bereits durch DasyLab gefilterte EMGSignal gleichgerichtet. Anschließend musste mit einem Cursor visuell die Baseline vor und nach der sichtbaren Innervationsphase markiert werden (Abb. 32). Durch die gesetzten Markierungen vor der sichtbaren Innervationsphase wurde ein mittleres Grundrauschen des EMG-Signals als Baseline zur Innervationsphase abgegrenzt. Sobald dieses mittlere Grundrauschen, wie oben definiert, für 100 ms um mehr als drei Standardabweichungen anstieg, wurde dieser Zeitpunkt als EMG-Onset festgelegt. Die gesetzten Markierungen nach der sichtbaren Innervationsphase stellten den EMG-Offset dar, der allerdings in dieser Untersuchung nicht weiter berücksichtigt wurde. 


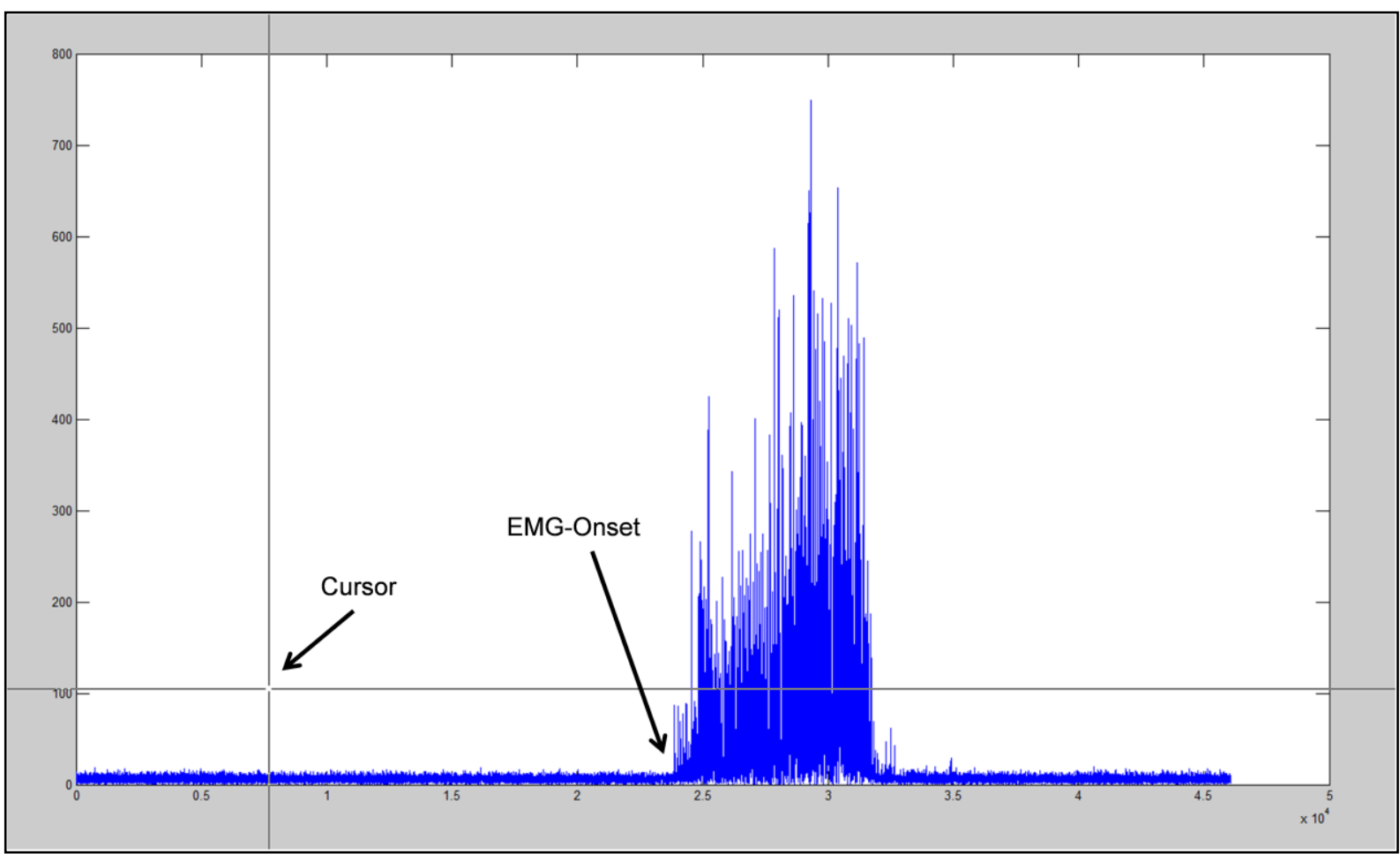

Abb. 32 Beispielhafte Darstellung eines gleichgerichteten EMG-Signals in MatLab eines Probanden zur Erfassung des EMG-Onset (Cursor = zeigt die zu setzende Markierung der Baseline, EMG-Onset = zeigt den ersten Zeitpunkt der Innervationsphase)

Nachdem der absolute EMG-Onset berechnet wurde, konnte anschließend der relative EMG-Onset prozentual von der maximalen Beweglichkeit errechnet werden. Der absolute EMG-Onset wurde in MatLab als Winkelgrad $\left[{ }^{\circ}\right]$ und der relative EMG-Onset in Prozent (\%) berechnet. Der MatLab-Code zur Berechnung der EMG-Parameter ist im Anhang dargestellt (S. 184).

\subsubsection{Treatment}

Die Probanden führten ein zehnwöchiges Dehntraining als statische Eigendehnung (dreimal pro Woche, dreimal 60 Sekunden pro Beinseite) an der ischiocruralen Muskulatur durch. Die Probanden bekamen eine konkrete schriftliche Anweisung, wie die Übung durchgeführt werden sollte (Abb. 33). 


\section{Ausgangsposition:}

Der Oberkörper ist aufrecht. Das Standbein ist gestreckt und das zu dehnende Bein wird ebenfalls gestreckt mit der Ferse auf einer erhöhten Unterlage abgelegt (zum Beispiel auf einem Stuhl). Der Fuß des zu dehnenden Beines soll ganz locker bleiben. Beide Hände werden auf dem zu dehnenden Oberschenkel abgestützt und halten so das Knie gestreckt (linkes Bild). Um besser das Gleichgewicht auf einem Bein zu halten, kann sich an einem Hilfsmittel festgehalten werden (zum Beispiel an einem einem Stock).
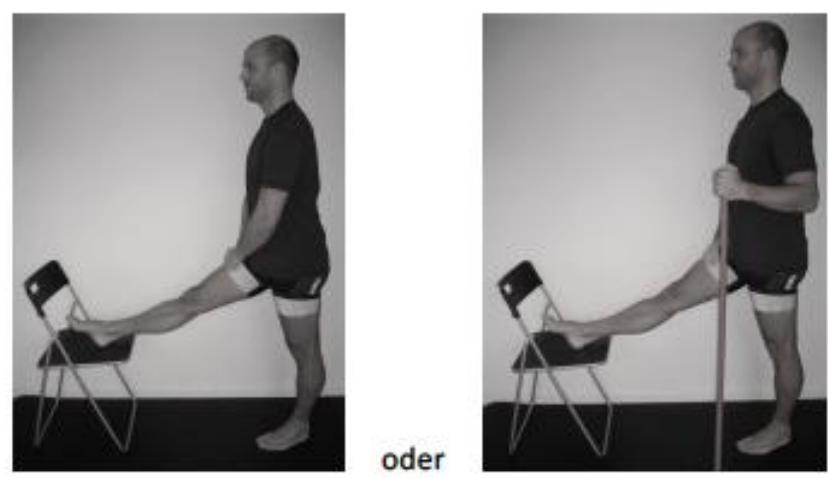

\section{Endposition:}

Der Rücken ist gerade und der Oberkörper wird nach vorne unten bewegt. Der Blick ist auf den vorderen Fuß gerichtet. Beide Hănde werden auf dem zu dehnenden Oberschenkel abgestützt und halten so das Knie gestreckt. Der Oberkörper ist so weit nach vorne gelehnt, bis eine Dehnung in der Oberschenkelrückseite zu spüren ist.

\section{Die Dehnung wird bei beiden Beinen jeweils 3 mal 60 Sekunden gehalten.}
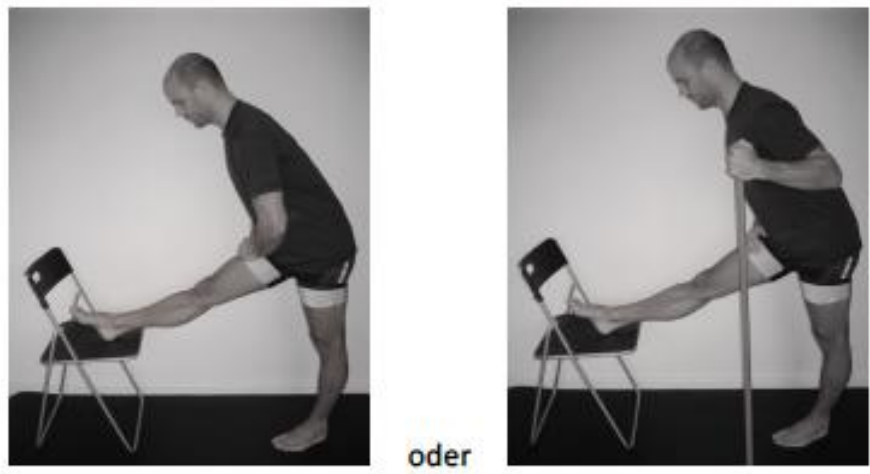

Intensităt:

Die Intensităt der Dehnung in der Endposition wird in der folgenden Tabelle gezeigt. Es sollte eine intensive Dehnung der Oberschenkelrückseite zu spüren sein (Skalenwert 7-8).

\begin{tabular}{|c|c|c|c|c|c|c|c|c|c|c|}
\hline \multirow{3}{*}{ Intensität } & \multicolumn{7}{|c|}{$\begin{array}{l}\text { kein } \\
\text { Dehnungsschmerz }\end{array}$} & \multicolumn{3}{|c|}{$\begin{array}{r}\text { unerträglicher } \\
\text { Dehnungsschmerz }\end{array}$} \\
\hline & 1 & 2 & 3 & 4 & 5 & 6 & 7 & 8 & 9 & 10 \\
\hline & \multicolumn{2}{|c|}{ etwas spürbar } & \multicolumn{2}{|c|}{ wenig intensiv } & \multicolumn{2}{|c|}{ etwas intensiv } & \multicolumn{2}{|c|}{ intensiv } & \multicolumn{2}{|c|}{ sehr intensiv } \\
\hline
\end{tabular}


Die Belastungsparameter orientierten sich an Trainingsempfehlungen für ältere Erwachsene des American College of Sports Medicine (Feland et al., 2001; Garber et al., 2011). Eine Dehnübung sollte laut Garber et al. (2011) zwei- bis viermal wiederholt werden. Feland et al. (2001) kamen zu dem Ergebnis, dass eine Trainingsmaßnahme mit 60-sekündiger Dehnung bei älteren Erwachsenen eine größere Verbesserung der Bewegungsreichweite zur Folge hat, als eine gleiche Trainingsmaßnahme mit 15- oder 30-sekündiger Dehnung. Darüber hinaus orientierten sich die Belastungsparameter an den Ergebnissen des vorausgegangenen systematischen Reviews. Das Treatment beschränkte sich auf eine statische Eigendehnung mit der Begründung, dass für Personen in einem höheren Lebensalter eine statische Eigendehnung einfacher als andere Dehnmethoden auszuführen ist. Außerdem sollte durch eine einfach auszuführende Dehnübung eine höhere Compliance der Probanden erreicht werden. Auch sind die Effekte hinsichtlich einer Verbesserung der Beweglichkeit zwischen den verschiedenen Dehnmethoden vergleichbar (Decoster et al., 2005). Bei der Durchführung der Dehnübung standen die Probanden vor einem Stuhl und legten das zu dehnende Bein gestreckt auf dem Stuhl ab. Sie sollten den Oberkörper so weit nach vorne unten neigen, bis in der Oberschenkelrückseite ein intensiver Dehnungsschmerz zu spüren war, der auf einer 10-stufigen Schmerzskala im Bereich 7 bis 8 kategorisiert wurde (Abb. 33). Die Probanden hatten die Wahl, ob sie sich freistehend dehnen, oder ob sie sich mit einem Stock abstützen, um das Gleichgewicht besser halten zu können. Nach Rücksprache mit den Probanden führten diese die Dehnübung alle freistehend ohne Hilfsmittel aus.

Während des zehnwöchigen Treatments führten die Probanden ein Aktivitätsprotokoll, in dem sie für jede Woche ihr Dehntraining dokumentierten. Das Aktivitätsprotokoll diente in Anlehnung an Cipriani et al. (2003) gleichzeitig zur Überprüfung der Compliance. Die Probanden wurden gebeten, die Dehnübung dreimal pro Woche durchzuführen, wobei immer ein Tag dazwischen Pause sein sollte. Die älteren Erwachsenen kamen ein- bis zweimal pro Woche in die Präventivsportgruppe und dehnten dort unter Aufsicht und mindestens einmal zu Hause. Bei den jüngeren Erwachsenen handelte es sich um Sportstudenten, 
mit denen über den Interventionszeitraum einmal pro Woche ein Treffen vereinbart wurde, um die Trainingsmaßnahme zu überprüfen. Des Weiteren wurden die Probanden gebeten, ihre Lebensgewohnheiten über den Interventionszeitraum nicht zu ändern. Sie sollten demnach keine neuen Sportarten beginnen und auch ihre körperlichen sowie sportlichen Aktivitäten so beibehalten wie es vor dem Interventionszeitraum der Fall war.

\subsubsection{Untersuchungsablauf}

Der Untersuchungszeitraum betrug insgesamt 12 Wochen (Abb. 34). Die ersten zwei Wochen dienten den Probanden an zwei separaten Terminen einer Gewöhnungsphase an die Testsituation. Während diesen Terminen wurde den Probanden ausführlich die Studie erklärt und etwaige Fragen wurden beantwortet. Die Termine wurden außerdem genutzt, um die Reliabilität der abhängigen Variablen zu bestimmen (vgl. S. 111). Anschließend erfolgte die 10-wöchige Trainingsphase.

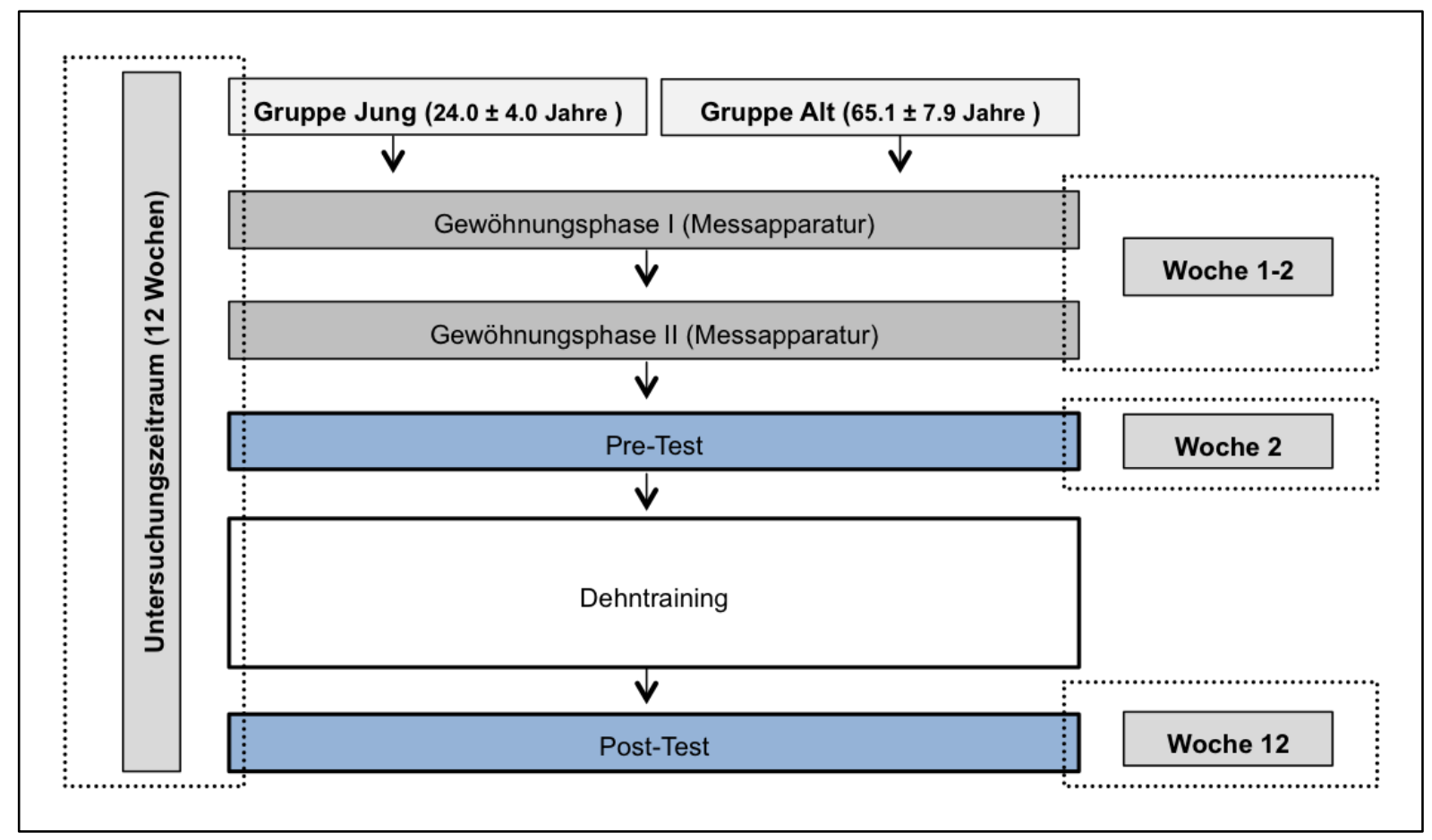

Abb. 34 Zeitlicher Untersuchungsablauf 


\subsubsection{Gewöhnungsphase}

Die Gewöhnungsphase sollte den Probanden dazu dienen, sich mit der Testsituation insgesamt vertraut zu machen und den ASLRT kennenzulernen. Da eine maximale Muskeldehnung insbesondere stark von der subjektiv wahrgenommenen Schmerzempfindung abhängig ist, sollten sich die Probanden innerhalb der zwei Termine möglichst nah an ihre individuelle maximale Beweglichkeit herantasten. Innerhalb der Gewöhnungsphase wurde die Testsituation genauso organisiert, wie sie auch während der Datenaufnahme zu den Testzeitpunkten durchgeführt wurde. Zum ersten Termin der Gewöhnungsphase bekamen die Probanden ein Aufklärungsschreiben ausgehändigt und unterschrieben eine Einverständniserklärung. Weiterhin wurden per Fragebogen die anthropometrischen Parameter und der aktuelle Gesundheitszustand abgefragt. Nachdem die Probanden den Fragebogen ausgefüllt hatten, wurde für die Berechnung des Drehmomentes, als Parameter der Dehnungsspannung, die Beinlänge von Fußboden bis Trochanter major gemessen.

\subsubsection{Testablauf}

Der Ablauf während der Termine zur Datenaufnahme im gesamten Untersuchungszeitraum war zur Gewöhnungsphase sowie zu den Pre- und Post-Tests äquivalent, sodass nachfolgend stellvertretend nur ein Termin beschrieben wird.

Zu Beginn eines jeden Termins wurde in einem Protokoll notiert, ob die Probanden Muskelkater an der Oberschenkelrückseite hatten und ob sie sich in den letzten 48 Stunden gedehnt haben. Anschließend wurde das EMG vorbereitet. Nachdem das EMG-Signal mittels Frequenzanalyse auf Störsignale und das Roh-EMG visuell überprüft wurde, erfolgte ein dreiminütiges Aufwärmen auf einem Fahrradergometer (Ergo-Fit, Ergo 1500 Cycle) mit $70 \mathrm{U} / \mathrm{min}$. bei einem Watt pro Kilogramm Körpergewicht ( $1 \mathrm{~W} / \mathrm{kg} \mathrm{KG}$ ). Die Intensität orientierte sich an dem Leistungsniveau der älteren Versuchsgruppe. Die ältere Versuchsgruppe wurde aus Teilnehmern einer Präventivsportgruppe rekrutiert. Diese absolvieren in jährlichem Abstand eine medizinische Untersuchung, in der ein Belastungs-EKG durchgeführt wird. Bei einigen Probanden stellte eine 
höhere Intensität als $1 \mathrm{~W} / \mathrm{kg}$ Körpergewicht bereits eine kritische Belastung des Herz-Kreislauf-Systems dar. Das Aufwärmen sollte für jeden Probanden einheitlich ablaufen. Aus diesem Grund wurde diese Intensität sowohl für die Untersuchungsgruppe der älteren als auch der jüngeren Erwachsenen gleichermaßen gewählt. Nachdem Aufwärmen erfolgte ein Pre-Stretching (statische Eigendehnung) der Oberschenkelrückseite $(6 \times 10 \mathrm{sec}$. pro Bein) um die Muskulatur in Anlehnung an Gajdosik et al. (2005) auf eine maximale Dehnung vorzubereiten. Das Pre-Stretching wurde mit der gleichen Übung wie im späteren Treatment durchgeführt (vgl. Abb. 33). Knudson (2006) konnte zeigen, dass sich die Stiffness aufgrund einer Erwärmung der Muskulatur beziehungsweise eines Warm-Up-Effektes verändert. Um diesen Effekt zu kontrollieren, führten die Probanden im Rahmen der hier vorliegenden Studie vor der Datenerhebung ein standardisiertes Aufwärmen durch. Außerdem wurde die Raumtemperatur möglichst konstant gehalten (Temperatur Pre-Test: $22.1 \pm 0.9{ }^{\circ} \mathrm{C}$, Temperatur Post-Test: $22.0 \pm 1.2^{\circ} \mathrm{C}$ ). Direkt im Anschluss an das PreStretching wurden die Probanden auf dem ASLRT positioniert (Abb. 21).

Die Probandenpositionierung wurde standardisiert vorgenommen und in einem Testprotokoll festgehalten. Nachdem der Proband auf dem ASLRT positioniert wurde, erfolgte die Datenaufnahme. Hierzu sollte der Proband den Hebelarm zweimal mit einer Geschwindigkeit von $1.5 \% \mathrm{sec}$. hoch- und mit einer Geschwindigkeit von $3.75 \% \mathrm{sec}$. wieder herunterfahren. Die Probanden wurden angehalten, den Hebelarm soweit hochzufahren beziehungsweise die Oberschenkelrückseite so lange zu dehnen, bis keine weitere Dehnung aufgrund eines intensiven Dehnungsschmerzes mehr möglich war. Im Wortlaut wurde den Probanden gesagt: „Bitte fahre den Hebelarm soweit hoch, bis kein weiteres Hochfahren mehr möglich ist. Dann gebe mit der linken Hand ein Zeichen und fahre anschließend den Hebelarm wieder in die Ausgangsposition zurück." Insgesamt sollten die Probanden darauf achten, dass der Hebelarm möglichst durchgängig bewegt wurde. Den Probanden wurde die Dehnintensität beziehungsweise der Dehnungsschmerz visualisiert, indem auf einer Schmerzskala von 0 bis $10 \quad(0=$ kein Dehnungsschmerz; $10=$ unerträglicher Dehnungsschmerz) während der Dehnung eine 8 - 9 (starker Dehnungsschmerz) erreicht werden sollte. Nachdem der Hebelarm in die Ausgangsposition heruntergefah- 
ren wurde, sollte der Proband auf einer $100 \mathrm{~mm}$-Schmerzskala sein subjektives Schmerzempfinden markieren. Außerdem wurde vermerkt, wo der Proband den Dehnungsschmerz primär empfunden hat.

\subsubsection{Hypothesen}

Nachfolgend werden die zu überprüfenden Hypothesen formuliert, die jeweils als Hypothesenpaare dargestellt werden. Hierbei wird die Nullhypothese vorangestellt. Die Hypothesen werden in biomechanische sowie neurophysiologische Parameter unterteilt und fortlaufend durchnummeriert.

\subsubsection{Biomechanische Parameter}

H0.1: Es besteht kein signifikanter Unterschied in der Veränderung der maximalen Beweglichkeit zwischen jüngeren und älteren Erwachsenen durch ein zehnwöchiges standardisiertes Dehntraining.

H1.1: Es besteht ein signifikanter Unterschied in der Veränderung der maximalen Beweglichkeit zwischen jüngeren und älteren Erwachsenen durch ein zehnwöchiges standardisiertes Dehntraining.

H0.2: Es besteht kein signifikanter Unterschied in der Veränderung der maximalen Dehnungsspannung zwischen jüngeren und älteren Erwachsenen durch ein zehnwöchiges standardisiertes Dehntraining.

H1.2: Es besteht ein signifikanter Unterschied in der Veränderung der maximalen Dehnungsspannung zwischen jüngeren und älteren Erwachsenen durch ein zehnwöchiges standardisiertes Dehntraining.

H0.3: Es besteht kein signifikanter Unterschied in der Veränderung der passiven-elastischen Stiffness zwischen jüngeren und älteren Erwachsenen durch ein zehnwöchiges standardisiertes Dehntraining. 
H1.3: Es besteht ein signifikanter Unterschied in der Veränderung der passiven-elastischen Stiffness zwischen jüngeren und älteren Erwachsenen durch ein zehnwöchiges standardisiertes Dehntraining.

H0.4: Es besteht kein signifikanter Unterschied in der Veränderung der passiven-elastischen Energie zwischen jüngeren und älteren Erwachsenen durch ein zehnwöchiges standardisiertes Dehntraining.

H1.4: Es besteht ein signifikanter Unterschied in der Veränderung der passiven-elastischen Energie zwischen jüngeren und älteren Erwachsenen durch ein zehnwöchiges standardisiertes Dehntraining.

\subsubsection{Neurophysiologische Parameter}

H0.5: Es besteht kein signifikanter Unterschied in der Veränderung des absoluten EMG-Onset zwischen jüngeren und älteren Erwachsenen durch ein zehnwöchiges standardisiertes Dehntraining.

H1.5: Es besteht ein signifikanter Unterschied in der Veränderung des absoluten EMG-Onset zwischen jüngeren und älteren Erwachsenen durch ein zehnwöchiges standardisiertes Dehntraining.

H0.6: Es besteht kein signifikanter Unterschied in der Veränderung des relativen EMG-Onset zwischen jüngeren und älteren Erwachsenen durch ein zehnwöchiges standardisiertes Dehntraining.

H1.6: Es besteht ein signifikanter Unterschied in der Veränderung des relativen EMG-Onset zwischen jüngeren und älteren Erwachsenen durch ein zehnwöchiges standardisiertes Dehntraining.

\subsubsection{Statistik}

Die statistischen Berechnungen wurden mit SPSS (IBM Corp., Version 23) durchgeführt. Es erfolgte zunächst eine Überprüfung der Voraussetzung zur Durchführung der statistischen Analysen. Alle abhängigen Variablen waren in- 
tervallskaliert und wurden auf Normalverteilung mittels Kolmogorov-SmirnovTest geprüft. Die abhängigen Variablen sind normalverteilt. Die Homogenität der Varianzen wurde mittels Levene-Test und die Sphärizität mittels MauchlyTest ermittelt.

Unterschiede der abhängigen Variablen zwischen den Altersgruppen im Vortest wurden mit unabhängigen t-Tests berechnet. Wurde eine Varianzheterogenität festgestellt, so wurden die Ergebnisse des t-Tests mit Welch-Korrektur verwendet.

Um den Einfluss des Lebensalters auf die Veränderung der abhängigen Variablen zu bestimmen, wurden zweifaktorielle Varianzanalysen mit Messwiederholung gerechnet. War die Voraussetzung der Sphärizität nicht erfüllt, so wurden die Ergebnisse durch eine Korrektur nach Greenhouse-Geisser verwendet.

Die Berechnung von altersspezifischen Veränderungen der abhängigen Variablen zwischen Pre- und Post-Test erfolgte mittels abhängigen t-Tests.

Das Signifikanzniveau wurde mit einem $\alpha$-Niveau von $p \leq .05$ festgelegt. Die Ergebnisse werden dargestellt als Mittelwert \pm Standardabweichung. Weiterhin werden signifikante Ergebnisse mit Effektstärken (partielles $\eta^{2}$ ) angegeben. Die Interpretation der Effektstärken erfolgte nach Cohen (1988): $\geq .01=$ kleiner Effekt, $\geq .06=$ mittlerer Effekt, $\geq .14=$ großer Effekt.

\subsection{Ergebnisse}

Nachfolgend werden die Ergebnisse der empirischen Untersuchung dargestellt. Zunächst werden die Ergebnisse der ANOVA in deskriptiver Form gezeigt. Anschließend erfolgt die Ergebnisdarstellung getrennt zu den einzelnen abhängigen Variablen. 
Die abhängigen Variablen unterschieden sich zum Vortest nicht signifikant zwischen den Altersgruppen: $\mathrm{ROM}_{\max }(t(23)=1.04, p=.31) ; \mathrm{Nm}_{\max }(t(23)=0.78$, $p=.44) ; \operatorname{PES}(t(23)=0.05, p=.96) ; \operatorname{PEE}(t(23)=1.35, p=.19) ; \quad \mathrm{EMG}_{\text {onset }}$ $\left(t(16)=-0.09, \quad p=.93 ; \quad \mathrm{EMG}_{\text {onset-\% }}(t(16)=-0.43, \quad p=.67), \quad\right.$ Schmerzskala $(t(23)=0.91, p=.37)$. Die Ergebnisse der Varianzanalyse sind in Tabelle 16 dargestellt.

Tab. 16 Ergebnisse der ANOVA und Messwerte zu Pre- und Post-Test $\left(R O M_{\max }=\operatorname{maxi}\right.$ male Beweglichkeit, $N m_{\max }=$ maximale Dehnungsspannung, $P E S=$ passive elastische Stiffness, $P E E=$ passive-elastische Energie, $E M G_{\text {onset }}=$ Gelenkwinkel beim EMG-Onset; $E M G_{\text {onset }-\%}=E M G$-Onset prozentual von $R O M_{\text {max. }}$. ${ }^{* *}=p<.001$ zwischen Pre- und Post-Test, ${ }^{*}=p<.05$ zwischen Pre- und PostTest)

\begin{tabular}{|c|c|c|c|c|c|}
\hline & Jung & Alt & ANOVA & $\mathbf{F}$ & p \\
\hline $\mathrm{ROM}_{\max .}\left({ }^{\circ}\right)$ & $\mathrm{n}=13$ & $\mathrm{n}=12$ & Alter & 1.25 & ns \\
\hline Pre-Test & $93.00 \pm 15.44$ & $86.70 \pm 14.90$ & Zeit & 56.33 & $<.001$ \\
\hline Post-Test & $101.40 \pm 13.01^{* *}$ & $94.72 \pm 15.65^{\star *}$ & Alter $x$ Zeit & $<1.0$ & ns \\
\hline $\mathrm{Nm}_{\max .}(\mathrm{Nm})$ & $n=13$ & $\mathrm{n}=12$ & Alter & $<1.0$ & ns \\
\hline Pre-Test & $103.11 \pm 45.35$ & $90.64 \pm 33.07$ & Zeit & 57.99 & $<.001$ \\
\hline Post-Test & $126.41 \pm 43.11^{\star *}$ & $108.06 \pm 36.11^{* *}$ & Alter $x$ Zeit & 1.21 & ns \\
\hline $\operatorname{PES}\left(\mathrm{Nm} /{ }^{\circ}\right)$ & $\mathrm{n}=13$ & $\mathrm{n}=12$ & Alter & $<1.0$ & ns \\
\hline Pre-Test & $0.62 \pm 0.17$ & $0.67 \pm 0.18$ & Zeit & 4.80 & $<.05$ \\
\hline Post-Test & $0.58 \pm 0.15$ & $0.62 \pm 0.13$ & Alter $x$ Zeit & $<1.0$ & ns \\
\hline PEE ('Nm) & $n=13$ & $\mathrm{n}=12$ & Alter & 1.46 & ns \\
\hline Pre-Test & $3484.84 \pm 1911.97$ & $2874.02 \pm 1348.58$ & Zeit & 30.25 & $<.001$ \\
\hline Post-Test & $4441.72 \pm 1835.88^{* *}$ & $3324.28 \pm 1644.19^{*}$ & Alter $x$ Zeit & 3.92 & ns \\
\hline
\end{tabular}


Tab. 16 (Fortsetzung)

\begin{tabular}{|c|c|c|c|c|c|}
\hline EMG onset $\left({ }^{\circ}\right)^{\#}$ & $\mathrm{n}=10$ & $\mathrm{n}=8$ & Alter & $<1.0$ & ns \\
\hline Pre-Test & $68.66 \pm 17.86$ & $69.19 \pm 5.21$ & Zeit & $<1.0$ & ns \\
\hline Post-Test & $68.84 \pm 18.13$ & $70.67 \pm 4.83$ & Alter $x$ Zeit & $<1.0$ & ns \\
\hline $\mathrm{EMG}_{\text {onset- } \%}(\%)^{\#}$ & $\mathrm{n}=10$ & $n=8$ & Alter & $<1.0$ & ns \\
\hline Pre-Test & $74.33 \pm 16.57$ & $77.30 \pm 11.64$ & Zeit & 8.8 & $<.01$ \\
\hline Post-Test & $68.24 \pm 17.03^{*}$ & $71.79 \pm 6.07$ & Alter $x$ Zeit & $<1.0$ & ns \\
\hline
\end{tabular}

\# Statistische Angaben nur von Probanden, die bei Pre- und Post-Test eine EMG-Aktivität während der Muskeldehnung zeigten (Erläuterungen siehe Text)

\subsubsection{Biomechanische Parameter}

Für $\mathrm{ROM}_{\max }$. konnte ein signifikanter Haupteffekt Zeit mit einem großen Effekt errechnet werden $\left(F(1,23)=56.33, p<.001\right.$, partielles $\left.\eta^{2}=.71\right)$. Es liegt keine signifikante Alter versus Zeit Interaktion vor $(F<1.0, p=.86)$. Die junge Altersgruppe erreichte $z u$ beiden Messzeitpunkten höhere $\mathrm{ROM}_{\text {max. }}$-Werte gegenüber der alten Altersgruppe (Tab. 16). Der Haupteffekt Alter wurde aber nicht signifikant $(F(1,23)=1.25, p=.28)$. Die alte Altersgruppe zeigte eine ähnlich große Veränderung der maximalen Bewegungsreichweite zwischen Pre- und Post-Test wie die junge Altersgruppe (Abb. 35).

Für $\mathrm{Nm}_{\max }$. konnte ein signifikanter Haupteffekt Zeit mit einem großen Effekt errechnet werden $\left(F(1,23)=57.99, p<.001\right.$, partielles $\left.\eta^{2}=.72\right)$. Es liegt keine signifikante Alter versus Zeit Interaktion vor $(F(1,23)=1.21, p=.28)$. Die junge Altersgruppe erreichte zu beiden Messzeitpunkten höhere $\mathrm{Nm}_{\max }$-Werte gegenüber der alten Altersgruppe (Tab. 16). Der Haupteffekt Alter wurde aber nicht signifikant $(F<1.0, p=.34)$. Die junge Altersgruppe steigerte die maximale Dehnungsspannung mehr als die alte Altersgruppe (Abb. 35). 
Für PES konnte ein signifikanter Haupteffekt Zeit mit einem großen Effekt errechnet werden $\left(F(1,23)=4.80, p=<.05\right.$, partielles $\left.\eta^{2}=.19\right)$. Es liegt keine signifikante Alter versus Zeit Interaktion vor $(F<1.0, p=.75)$. Die junge Altersgruppe erreichte zu beiden Messzeitpunkten geringere PES-Werte gegenüber der alten Altersgruppe (Tab. 16). Der Haupteffekt Alter wurde aber nicht signifikant $(F<1.0, p=.51)$. Die alte Altersgruppe verringerte die Stiffness zwischen $20^{\circ}$ - und $50^{\circ}$-Hüftflexionswinkel mehr als die junge Altersgruppe (Abb. 35).

Für PEE konnte ein signifikanter Haupteffekt Zeit mit einem großen Effekt errechnet werden $\left(F(1,23)=30.25, p<.001\right.$, partielles $\left.\eta^{2}=.59\right)$. Es liegt keine signifikante Alter versus Zeit Interaktion vor $(F(1,23)=3.92, p=.06)$. Die junge Altersgruppe erreichte zu beiden Messzeitpunkten höhere PEE-Werte gegenüber der alten Altersgruppe (Tab. 16). Der Haupteffekt Alter wurde aber nicht signifikant $(F(1,23)=1.46, p=.24)$. Die junge Altersgruppe erreichte eine größere prozentuale Veränderung der passiv-elastischen Energie gegenüber der alten Altersgruppe (Abb. 35).

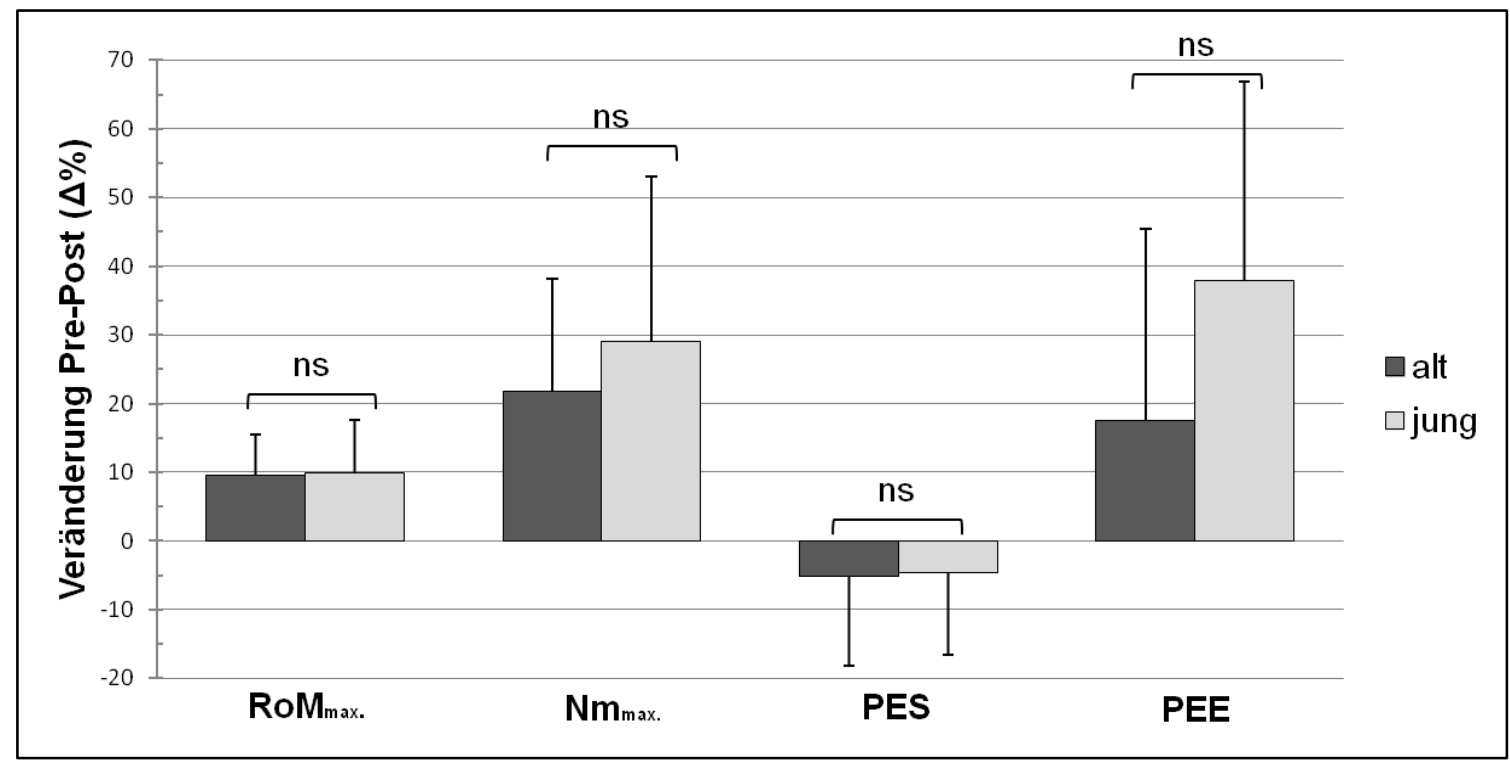


Abb. 35 Prozentuale Veränderung der biomechanischen Parameter zwischen Pre- und Post-Test $($ ROMmax. = maximale Beweglichkeit, Nmmax. = maximale Dehnungsspannung, $P E S=$ passive-elastische Stiffness, $P E E=$ passive-elastische Energie, $n s=$ kein signifikanter Unterschied zwischen der alten und jungen Untersuchungsgruppe)

\subsubsection{Neurophysiologische Parameter}

Für die statistische Analyse der EMG-Parameter ( $E M G_{\text {onset }}$ und $E M G_{\text {onset-\%) }}$ ) wurden nur die Probanden herangezogen, die zum Pre- und Post-Test einen EMG-Onset zeigten. Bei drei jungen und vier alten Probanden zeigte sich mindestens bei einem Messzeitpunkt kein EMG-Onset (Tab. 16).

Für $E M_{\text {onset }}$ konnte kein signifikanter Haupteffekt Zeit errechnet werden $(F<1.0, p=.66)$. Es liegt keine signifikante Alter versus Zeit Interaktion vor $(F<1.0, \quad p=.73)$. Der Haupteffekt Alter wurde nicht signifikant $(F<1.0$, $p=.86)$. Die ältere Altersgruppe zeigte eine größere prozentuale Veränderung des absoluten EMG-Onset gegenüber der jungen Altersgruppe (Abb. 36).

Für $E_{M G_{\text {onset-\% }}}$ konnte ein signifikanter Haupteffekt Zeit mit einem großen Effekt errechnet werden $\left(F(1,16)=8.8, p<.01\right.$, partielles $\left.\eta^{2}=.35\right)$. Es liegt keine signifikante Alter versus Zeit Interaktion vor $(F<1.0, p=.88)$. Die junge Altersgruppe erreichte zu beiden Messzeitpunkten niedrigere $E M G_{\text {onset-\%-Werte }}$ gegenüber der alten Altersgruppe (Tab. 16). Der Haupteffekt Alter wurde aber nicht signifikant $(F<1.0, p=.62)$. Die junge Altersgruppe erreichte eine etwas größere prozentuale Veränderung des relativen EMG-Onset gegenüber der alten Altersgruppe (Abb. 36). 


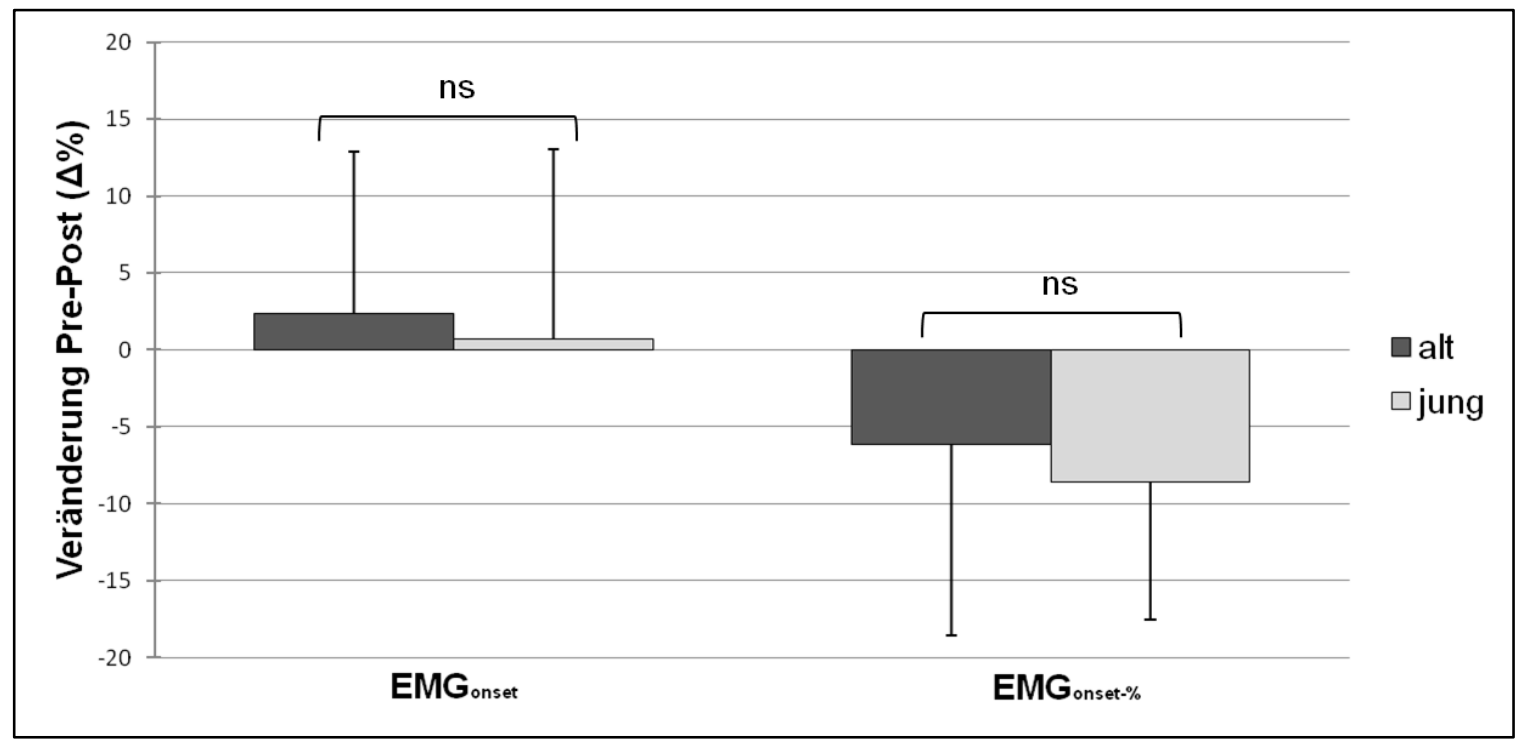

Abb. 36 Prozentuale Veränderung der neurophysiologischen Parameter zwischen Pre- und Post-Test (EMGonset = Gelenkwinkel beim EMG-Onset; EMGonset-\% = EMGOnset prozentual von ROMmax., ns = kein signifikanter Unterschied zwischen der alten und jungen Untersuchungsgruppe)

\subsubsection{Schmerzskala}

Die parallel erhobenen Daten der Schmerzskala ergaben eine signifikante Veränderung bei der jungen Altersgruppe zwischen Vor- und Nachtest von $86 \pm 14 \mathrm{~mm}$ um $9.5 \%$ auf $93 \pm 10 \mathrm{~mm}(t(12)=-2.4, p<.05)$. Bei der alten Altersgruppe veränderten sich die Angaben auf der Schmerzskala nicht signifikant von $82 \pm 10 \mathrm{~mm}$ um $8.6 \%$ auf $88 \pm 5 \mathrm{~mm}(t(11)=-2.04, p=.07)$.

\subsection{Diskussion}

In der empirischen Studie wurden biomechanische und neurophysiologische Anpassungserscheinungen der ischiocruralen Muskulatur durch ein standardisiertes zehnwöchiges Dehntraining bei jüngeren und älteren Erwachsenen untersucht. Nachfolgend werden die Ergebnisse in Bezug zu den Hypothesen getrennt nach biomechanischen und neurophysiologischen Parametern ausführlich diskutiert. 


\subsubsection{Biomechanische Parameter}

In Bezug auf die ersten beiden formulierten Hypothesen im Rahmen der untersuchten abhängigen Variablen kommt die durchgeführte Untersuchung zu dem Ergebnis, dass Hypothese $\mathrm{H} 1.1$ abgelehnt wird: Es besteht kein signifikanter Unterschied in der Veränderung der maximalen Beweglichkeit zwischen jüngeren und älteren Erwachsenen durch ein zehnwöchiges standardisiertes Dehntraining.

Des Weiteren wird die Hypothese H1.2 abgelehnt: Es besteht kein signifikanter Unterschied in der Veränderung der maximalen Dehnungsspannung zwischen jüngeren und älteren Erwachsenen durch ein zehnwöchiges standardisiertes Dehntraining.

Bezüglich der maximalen Beweglichkeit erreichten beide Altersgruppen ähnliche prozentuale Zuwachsraten. Die junge Altersgruppe hingegen erreichte bei der maximalen Dehnungsspannung eine höhere prozentuale Veränderung im Vergleich zur alten Altersgruppe. Kurzfristige Effekte nach einer Dehnung der Wadenmuskulatur bei älteren Personen zeigten bei Ryan et al. (2014), dass im Vergleich zu einer jüngeren Versuchsgruppe sowohl bei der maximalen Beweglichkeit als auch bei der maximalen Dehnungsspannung die ältere Versuchsgruppe stärkere Veränderungen aufwies. Ryan et al. (2014) führten die stärkeren Veränderungen der älteren Probanden auf eine altersabhängige Veränderung des Muskel-Sehnen-Gewebes zurück. Im Rahmen der hier vorliegenden langzeitigen Studie weist das Ergebnis vielmehr darauf hin, dass die junge Altersgruppe besser in der Lage war den Dehnungsschmerz zu tolerieren. Die maximale Beweglichkeit korreliert bei Ryan et al. (2014) mit der maximal zu ertragenden Dehnungsspannung $(r=0.7)$. In der vorliegenden Arbeit wurde der Zusammenhang zwischen der prozentualen Veränderung der maximalen Beweglichkeit und der maximalen Dehnungsspannung mit einem PearsonKorrelationskoeffizienten berechnet. Sowohl für die alte Altersgruppe $\left(r_{\text {alt }}(11)=0.7, p<.05\right)$ als auch für die junge Altersgruppe $\left(r_{\text {jung }}(10)=0.9\right.$, $p<.001)$ errechnete sich nach der Definition von Cohen (1988) ein großer Zusammenhang. Der Korrelationskoeffizient der alten Altersgruppe deckt sich in etwa mit dem Korrelationskoeffizienten nach einer akuten Dehnungsinterventi- 
on bei Ryan et al. (2014). Dennoch zeigte sich bei den jüngeren Erwachsenen ein höherer Zusammenhang zwischen der maximalen Beweglichkeit und der maximalen Dehnungsspannung. Dieses Ergebnis deutet darauf hin, dass die älteren Erwachsenen weniger an ihre individuelle Schmerzgrenze gegangen sind. Dafür spricht auch die Auswertung der $100 \mathrm{~mm}$-Schmerzskala. Die junge Altersgruppe wies höhere Werte auf der Schmerzskala auf und diese veränderten sich, im Gegensatz zur älteren Versuchsgruppe, signifikant zwischen Preund Post-Test.

Bezüglich der untersuchten passiven-elastischen Stiffness wird die Hypothese H1.3 abgelehnt: Es besteht kein signifikanter Unterschied in der Veränderung der passiven-elastischen Stiffness zwischen jüngeren und älteren Erwachsenen durch ein zehnwöchiges standardisiertes Dehntraining.

Die passive-elastische Stiffness beschreibt die muskelphysiologische Response während einer Muskeldehnung. Das Ergebnis zeigt, dass sich die Stiffness bei einem Hüftflexionswinkel zwischen $20^{\circ}$ und $50^{\circ}$ in beiden Altersgruppen verringerte. Die Veränderung wurde allerdings weder zwischen Pre- und PostTest noch zwischen den Altersgruppen signifikant. Ein ähnliches Ergebnis konnte bei einer Untersuchung von LaRoche und Connolly (2006) gezeigt werden, in der die errechnete Stiffness bei jungen Probanden bei einem Hüftflexionswinkel zwischen $50^{\circ}$ und $85^{\circ}$ nach einem vierwöchigen Dehntraining der ischiocruralen Muskulatur zwar verringert wurde, allerdings ohne signifikantem Unterschied zu einer Kontrollgruppe. Eine signifikante Verringerung der Stiffness der ischiocruralen Muskulatur bei einem Hüftflexionswinkel zwischen $20^{\circ}$ und $50^{\circ}$ nach einem vierwöchigen Dehntraining konnten nur Marshall et al. (2011) zeigen. Die Autoren geben hier allerdings zu bedenken, dass in ihrer Studie das Dehntraining einen ungewöhnlich hohen Umfang im Gegensatz zu anderen Studien hatte. Das Dehntraining umfasste insgesamt vier Übungen, die jeweils dreimal 30 Sekunden an fünf Tagen pro Woche durchgeführt wurden. Im Vergleich zu der hier vorliegenden Studie wurde nur eine Übung dreimal wöchentlich für dreimal 60 Sekunden durchgeführt. Bei Gajdosik et al. (2005) erhöhte sich die Stiffness in der letzten Hälfte der gesamten Dehnungsspannungskurve nach einem achtwöchigen Dehntraining der Wadenmuskulatur 
bei alten Probanden. Die Studienlage ist bezüglich der Stiffness uneinheitlich und wird kontrovers diskutiert. Konrad und Tilp (2014, S. 640) begründen dies mit unterschiedlich durchgeführten Studien hinsichtlich der Dehndauer und der Dehnintensität. Des Weiteren wird die Stiffness nicht einheitlich definiert. Oftmals werden standardisierte Winkelwerte der Dehnungsspannungskurve verwendet, die dann allerdings unterhalb der linearen Region der Dehnungsspannungskurve liegen (Knudson, 2006). Auch in der hier vorliegenden Studie wurde ein standardisierter Gelenkwinkelbereich zur Messung der Stiffness festgelegt, der möglicherweise nicht der linearen Region zuzuordnen ist. In zukünftigen Studien sollten daher keine absoluten Gelenkwinkelbereiche auf der Dehnungsspannungskurve gewählt werden, sondern individuelle Werte, die sich möglichst nah an der maximalen Beweglichkeit und somit in der linearen beziehungsweise elastischen Region befinden. Eine Möglichkeit wäre in Anlehnung an Reid und McNair (2004) die letzten $10 \%$ der gesamten Bewegungsreichweite als Stiffness zu definieren.

Bezüglich der untersuchten passiven-elastischen Energie wird die Hypothese H1.4 abgelehnt: Es besteht kein signifikanter Unterschied in der Veränderung der passiven-elastischen Energie zwischen jüngeren und älteren Erwachsenen durch ein zehnwöchiges standardisiertes Dehntraining.

Die Fläche unterhalb der Dehnungsspannungskurve repräsentiert neben der Stiffness einen weiteren elastischen Parameter, nämlich die passive-elastische Energie. Die Veränderung nach dem zehnwöchigen Dehntraining ergab eine signifikante Erhöhung zwischen Pre- und Post-Test bei beiden Altersgruppen. Der Unterschied zwischen den Altersgruppen wurde nicht signifikant. Das bedeutet, dass die Muskulatur in beiden Altersklassen nach einem langzeitigen Dehntraining in der Lage war, mehr passive-elastische Energie zu absorbieren. Die junge Altersgruppe war zu beiden Messzeitpunkten besser in der Lage, passive-elastische Energie zu absorbieren und auch die Veränderungen zwischen Pre- und Post-Test waren bei der jungen Altersgruppe größer. Bei Magnusson et al. (1997) wurden Langstreckenläufer mit normaler und geringerer Flexibilität der ischiocruralen Muskulatur hinsichtlich der Stiffness und der absorbierten Energie während einer Muskeldehnung untersucht. Im Ergebnis 
wurde festgestellt, dass die Muskulatur der Probanden mit geringerer Flexibilität weniger Energie absorbieren konnte und die Stiffness höher war als bei den Probanden mit normaler Flexibilität. Dies entspricht den Ergebnissen in der hier vorliegenden Studie, da auch hier die alte Versuchsgruppe eine geringere passive-elastische Energie sowie eine höhere passive-elastische Stiffness aufwies. Die Verbesserung der passiven-elastischen Energie in der jungen Altersgruppe war höher, dennoch konnte sich auch die alte Altersgruppe signifikant zwischen Pre- und Post-Test verbessern. Die Erhebung der elastischen Eigenschaften (passive-elastische Stiffness und passive-elastische Energie) der Muskulatur erfolgt in Abhängigkeit der Beweglichkeit und der Dehnungsspannung in dem jeweiligen Gelenkwinkel. Diese Abhängigkeit ist bei der Bewertung von Dehntrainingsstudien zu berücksichtigen. Das bedeutet, je höher die Beweglichkeit und die Dehnungsspannung ausgeprägt ist, desto mehr ist die Muskulatur in der Lage passive Energie zu absorbieren.

\subsubsection{Neurophysiologische Parameter}

Die neurophysiologischen Parameter wurden als EMG-Onset erhoben und unterteilen sich in einen absoluten und einen relativen EMG-Onset. Bezüglich der untersuchten EMG-Parameter wird die Hypothese H1.5 abgelehnt: Es besteht kein signifikanter Unterschied in der Veränderung des absoluten EMG-Onset zwischen jüngeren und älteren Erwachsenen durch ein zehnwöchiges standardisiertes Dehntraining.

Des Weiteren wird die Hypothese H1.6 abgelehnt: Es besteht kein signifikanter Unterschied in der Veränderung des relativen EMG-Onset zwischen jüngeren und älteren Erwachsenen durch ein zehnwöchiges standardisiertes Dehntraining.

Der absolute EMG-Onset unterschied sich nicht zwischen den Altersgruppen und veränderte sich nicht signifikant zwischen Pre- und Post-Test. Dieses Resultat stimmt mit Untersuchungen an der Wadenmuskulatur (Blazevich et al., 2014) sowie an der ischiocruralen Muskulatur (Magnusson et al., 1996) nach einem dreiwöchigen Dehntraining überein. Des Weiteren verringerte sich der relative EMG-Onset in beiden Altersgruppen zwischen Pre- und Post-Test. Ein 
unerwartetes Ergebnis ist, dass die Älteren einen späteren relativen EMGOnset im Vergleich zu den Jüngeren aufwiesen. Entgegen den hier vorliegenden Ergebnissen zeigten Ryan et al. (2014) nach einer einmaligen Dehnung der Wadenmuskulatur bei jüngeren und älteren Probanden, dass der relative EMG-Onset bei der älteren Versuchsgruppe früher einsetzte. Die Autoren spekulierten, dass möglicherweise eine höhere Stiffness bei den alten Probanden zu einer früheren Reflexantwort der Muskulatur führte. In der hier vorliegenden Studie wies die ältere Altersgruppe zwar eine höhere Stiffness auf, die allerdings keinen Einfluss auf den relativen EMG-Onset zu haben scheint. Es wird an dieser Stelle auch an die oben beschriebene Diskussion erinnert (vgl. S. 138), dass die Stiffness in Dehntrainingsstudien nicht einheitlich erhoben wird und deshalb die Stiffness bei der Interpretation von Studien differenzierter betrachtet werden muss (Konrad \& Tilp, 2014, S. 640). Bezüglich des EMGOnset wurde in der vorliegenden Studie, im Gegensatz zu Ryan et al. (2014), ein Warm-up und unmittelbar vor der Datenerhebung ein Pre-Stretching durchgeführt. Möglicherweise führte das Warm-up und das Pre-Stretching zu einer akuten Reduzierung der Stiffness in beiden Altersgruppen und beeinflusste so den EMG-Onset. Knudson (2006) beschreibt, dass sich die Stiffness aufgrund eines Warm-up-Effektes der Muskulatur verändern kann. Außerdem führt ein Pre-Stretching unmittelbar vor der Datenerhebung zu einer Verringerung der Dehnungsspannung, die bei Gajdosik et al. (2005, S. 975) als „tissue-forcerelaxation" beschrieben wird. Entgegen der Annahme, dass ein früherer relativer EMG-Onset aufgrund einer höheren Stiffness ausgelöst werden könnte, wird in der hier vorliegenden Studie ein späterer relativer EMG-Onset bei den älteren Probanden damit begründet, dass die jüngeren Probanden zum Preund Post-Test eine größere maximale Beweglichkeit erreichten. Der relative EMG-Onset ist abhängig von der maximalen Beweglichkeit. Die junge Versuchsgruppe erreichte bei Pre- und Post-Test eine höhere maximale Beweglichkeit als die alte Versuchsgruppe. Aufgrund einer geringeren maximalen $\mathrm{Be}-$ weglichkeit der alten Probanden, bei ähnlichem absoluten EMG-Onset in beiden Altersgruppen, kommt es aus diesem Grund zu einem späteren relativen EMG-Onset in der älteren Versuchsgruppe. 
Ähnlich wie in anderen Untersuchungen zeigten nicht alle Probanden einen EMG-Onset während der Muskeldehnung (Blazevich et al., 2012; McHugh et al., 1998; Ryan et al., 2014; Schönthaler \& Ohlendorf, 2002). Dies war sowohl bei der jungen als auch bei der alten Versuchsgruppe der Fall. Möglicherweise war die Dehnungsgeschwindigkeit mit $1.5 \% \mathrm{sec}$. während der passiven Dehnung auf dem apparativen Straight-Leg-Raise-Test zu niedrig, sodass es aufgrund unterschiedlicher Geschwindigkeitssensitivitäten der primären Endigungen der Muskelspindel teilweise zu keiner muskulären Reflexantwort kam. Die primären Endigungen der Muskelspindel reagieren während einer Dehnung sensibel auf die Geschwindigkeit einer Längenänderung (Matthews, 1981; 1964). Toft et al. (1989, S. 490) beschreiben in ihrer Untersuchung: „Stretch reflexes were not elicited since the stretch velocity was low $(<2 \mathrm{deg} / \mathrm{sec})$ ". Eine weitere Möglichkeit wäre, dass Probanden mit einer geringeren Stiffness auch geringere oder eben auch keine nennenswerten EMG-Aktivitäten aufweisen. Abellaneda, Guissard und Duchateau (2009) fanden eine höhere EMG-Aktivität bei Personen mit höherer Stiffness der Wadenmuskulatur gegenüber Personen mit geringerer Stiffness. Interessanterweise wiesen aber bei Magnusson et al. (1997) weder Probanden mit höherer als auch mit geringerer Stiffness der ischiocruralen Muskulatur ein EMG-Onset während eines passiven KneeExtension-Tests auf. Warum bei manchen Probanden ein EMG-Onset während einer Dehnung ausblieb, kann mit dieser Studie nicht eindeutig geklärt werden. Es bleibt festzuhalten, dass in der vorliegenden Studie, in Anlehnung an Magnusson et al. (1997, 1996) und Mc Hugh et al. (1998), der EMG-Onset keinen Einfluss auf die maximale Beweglichkeit bei jungen und alten Erwachsenen hatte.

\subsubsection{Methodendiskussion}

Abschließend wird die Methodik der empirischen Untersuchung kritisch betrachtet und diskutiert.

Es wurde ein quasi-experimentelles Untersuchungsdesign mit Vorher-NachherMessung an zwei Experimentalgruppen ohne Kontrollgruppen durchgeführt. 
Dass die Untersuchung ohne Kontrollgruppen durchgeführt wurde, könnte als Kritikpunkt gesehen werden. Eine Untersuchung mit Kontrollgruppe wird immer dann durchgeführt, wenn sichergestellt werden soll, dass die Wirkung einer Intervention ohne den Einfluss von Störvariablen stattgefunden hat. Nur mit einer Studie im Kontrollgruppendesign kann die interne Validität gewährleistet sein (Pospeschill, 2013, S. 83). Das Anliegen dieser Untersuchung war es, dass zwei Experimentalgruppen die gleiche Intervention durchführten, um die Unterschiede der Auswirkungen miteinander zu vergleichen. Aus diesem Grund wurde auf eine Kontrollgruppe verzichtet. Bei einer zusätzlichen Rekrutierung von zwei Kontrollgruppen hätte sich zudem die Stichprobengröße verdoppelt. Aufgrund der Schwierigkeit, Probanden über zehn Wochen an eine Untersuchung zu binden, wäre eine Verdoppelung der Anzahl der Versuchspersonen, insbesondere ältere Versuchspersonen ohne gesundheitliche Einschränkungen, in dieser Form schwierig gewesen. Aufgrund der Abwesenheit von Kontrollgruppen in der hier durchgeführten Untersuchung kann der Einfluss von möglichen Störvariablen nicht ausgeschlossen werden.

In Anlehnung an Pospeschill (2013, S. 173) sollten bei quasi-experimentellen Untersuchungsdesigns „... zur Steigerung der internen Validität mehr als zwei Messzeitpunkte durchgeführt werden“. Im Rahmen der hier durchgeführten Untersuchung wurde versucht zwei zusätzliche Zwischentests durchzuführen. Allerdings gab es bei der Mehrheit der Probanden Schwierigkeiten mit der Terminabstimmung für die Zwischentests, sodass mehrere Messzeitpunkte für alle Probanden in gleichen Zeitabständen nicht realisiert werden konnten. Aus diesem Grund erfolgte letztlich die Ergebnisdarstellung als Differenz zwischen Vorher- und Nachher-Messung. Mit der Möglichkeit, mehrere Messzeitpunkte über den gesamten Interventionszeitraum auszuwerten, hätte empirisch dargestellt werden können, ob die ältere Versuchsgruppe längere Zeit zur Anpassung an ein Dehntraining benötigt als die jüngere Versuchsgruppe.

Die Pre-Messung im Rahmen des Untersuchungsdesigns erfolgte erst nach einer Gewöhnungsphase. Bezüglich der internen Validität wurde versucht den 
Einfluss eines Testeffektes zu kontrollieren, indem vor der Pre-Messung die Probanden an zwei zusätzlichen Terminen den gesamten Testablauf durchliefen. Aufgrund der komplexen Testsituation mit der apparativen Messapparatur und den zusätzlichen Ableitungen zur Messung der elektromyographischen Signale erschien es sinnvoll, die Probanden an diese nicht alltägliche Testsituation heranzuführen. Diese Vorgehensweise sollte dazu dienen, zur eigentlichen Pre-Messung aussagekräftige Daten der Probanden als Baseline zu erheben. Um hinsichtlich der externen Validität in Anlehnung an Wilkening und Wilkening (1985, S. 16) eine Interaktion zwischen der unabhängigen Variable (Dehnungsintervention) und der Testung zu verhindern, wurden den Probanden bei der Pre-Messung die Messergebnisse nicht mitgeteilt. Dies sollte verhindern, dass Probanden mit etwas schlechteren Beweglichkeitsmaßen während der Interventionsphase beziehungsweise im Post-Test versuchen, bewusst bessere Leistungen zu erzeugen als Probanden die schon ein relativ gutes Ausgangsniveau hatten.

Ein wesentlicher Kritikpunkt bezüglich des Untersuchungsdesigns besteht in einer fehlenden Follow-up-Messung. Mit einer solchen Messung, nach Abschluss der Trainingsintervention, hätte die Stabilität beziehungsweise eine Veränderung der Anpassungseffekte beider Altersklassen zusätzlich erhoben werden können. Nach einer einmaligen Dehnungsintervention konnte gezeigt werden, dass eine Verbesserung der Beweglichkeit über 24 Stunden erhalten bleiben kann (de Weijer, Gorniak, \& Shamus, 2003). Inwiefern weitere biomechanische Anpassungseffekte nach einem langzeitigen Dehnungsprogramm stabil bleiben, beziehungsweise welche zeitlichen Veränderungen sich ergeben, sollte in weiteren Studien untersucht werden.

Ein weiterer Kritikpunkt könnte sein, dass im Rahmen der Datenerhebung nur das linke Bein zur Testung der abhängigen Variablen herangezogen wurde. Die Erhebung der abhängigen Variablen erfolgte an einem apparativen StraightLeg-Raise-Test (ASLRT). Diese Messapparatur lässt die Dehnung und die Datenaufnahme an beiden Beinseiten zu. Allerdings müsste hierzu ein Teil der 
Messapparatur umgebaut und der Proband neu positioniert werden. Aufgrund einer möglichst standardisierten und ökonomischen Testsituation wurde entschieden, nur das linke Bein zu testen. Das linke Bein wurde auch deshalb gewählt, da es während der Datenaufnahme gefilmt werden musste. Da das Bein nicht gegen ein unwillkürliches Beugen fixiert werden konnte, wurde es während der Dehnungsprozedur auf dem ASLRT mit einer Videokamera gefilmt und anschließend wurde visuell überprüft, ob das getestete Bein während der gesamten Dehnungsprozedur gestreckt war. Aus Platzgründen im Untersuchungsraum konnte die Videokamera nur auf der linken Seite aufgebaut werden. Wäre das rechte Bein auf dem ASLRT gedehnt worden, dann würde das Bein vom Hebelarm des ASLRT verdeckt werden und eine optimale Videoaufnahme des zu testenden Beines wäre nicht möglich gewesen.

Als nächster Diskussionspunkt soll auf die Probandenstichprobe eingegangen werden. Beide Untersuchungsgruppen galten als Freizeitsportler und wiesen keine offensichtlichen orthopädischen Einschränkungen auf. Auch gewährleisteten die a priori festgelegten Ein- und Ausschlusskriterien eine möglichst homogene Versuchsgruppe. Das Niveau der abhängigen Variablen zu Untersuchungsbeginn unterschied sich nicht signifikant zwischen den Versuchsgruppen. Allerdings startete die ältere Versuchsgruppe auf einem etwas niedrigeren Leistungsniveau in die Untersuchung. Des Weiteren wurden nur männliche Probanden rekrutiert, sich während der Rekrutierungsphase wesentlich mehr männliche als weibliche Probanden freiwillig zu der Untersuchung meldeten. Um die Untersuchungsgruppen möglichst homogen zu gestalten, wurde entschieden, auf weibliche Probanden zu verzichten. Bezugnehmend auf die rekrutierten Probanden mit den spezifischen Ein- und Ausschlusskriterien, beschränken sich die Untersuchungsergebnisse auf die hier untersuchte Personengruppe.

Die Untersuchung wurde nur mit einer Dehnübung und einer Dehnungsmethode an einer Muskelgruppe durchgeführt. Die ausgewählte Dehnübung sollte einfach und mit möglichst wenigen Freiheitgraden durchführbar sein. Dies soll- 
te die Compliance der Probanden erhöhen und mögliche Fehlerbilder vermeiden. Des Weiteren wurde die statische Eigendehnung ausgewählt, da bezüglich einer Verbesserung der Beweglichkeit diese Dehnmethode mit anderen vergleichbar ist. Die ischiocrurale Muskulatur wurde ausgewählt, da diese Muskelgruppe von beiden Untersuchungsgruppen mit der vorgegebenen Dehnübung ohne externe Hilfe beziehungsweise Hilfsmittel durchzuführen war. Eine andere Möglichkeit wäre gewesen, mehrere Dehnmethoden oder auch Dehnübungen im Rahmen der durchgeführten Intervention zu verbinden. Allerdings wäre dann der Trainingseffekt nicht auf eine konkrete Dehnmethode zurückzuführen. Somit beschränken sich die Ergebnisse nur auf die hier durchgeführte statische Eigendehnung an der ischiocruralen Muskulatur.

Die Positionierung der Oberflächenelektroden zur Ableitung der EMG-Signale während der Muskeldehnung im Pre- und Post-Test könnte einen weiteren Kritikpunkt darstellen. Es konnte nicht gewährleistet werden, dass die Elektroden im Post-Test exakt auf die gleiche Stelle geklebt wurden wie im Pre-Test. Hierzu hätte die Platzierung der Elektroden im Pre-Test mit einem Filzstift markiert und über den gesamten Untersuchungszeitraum von zehn Wochen nachgezeichnet werden müssen. Allerdings kann auch bei dieser Vorgehensweise nicht sichergestellt werden, dass die Markierungen immer exakt nachgezeichnet worden wären. Merletti, Rainoldi und Farina (2001, S. 22) konnten zeigen, dass es bei unterschiedlicher Elektrodenpositionierung zu entsprechend unterschiedlicher EMG-Signalcharakteristik kommt. Im Rahmen der hier durchgeführten empirischen Untersuchung sollte nicht die Signalcharakteristik untersucht werden, sondern ob die Muskulatur während der Dehnungsprozedur ein EMG-Onset aufwies. Aus diesem Grund erscheint die nicht exakt reproduzierte Elektrodenpositionierung vernachlässigbar zu sein. Da der Versuchsleiter im Pre- und Post-Test derselbe war und der Muskelbauch des M. biceps femoris sehr gut zu palpieren ist, kann davon ausgegangen werden, dass die Elektrodenpositionierung dennoch annähernd gleich war. Darüber hinaus war der Interelektrodenabstand bei beiden Messzeitpunkten identisch, da dieser durch die Form der Oberflächenelektroden vorgegeben war. 
Die Intensität während einer Muskeldehnung lässt sich nur schwer quantifizieren und ist aufgrund eines individuellen subjektiven Schmerzempfindens nicht optimal zu standardisieren. Dies stellt einen Kritikpunkt im Rahmen der durchgeführten Intervention dar. Im Vergleich zu anderen Studien, die keine Intensitätsangaben machten (vgl. Björklund et al., 2001; Sainz de Baranda \& Ayala, 2010; Ylinen et al., 2009) oder die Dehnintensität nicht quantifizierten (vgl. Johnson et al., 2014; LaRoche \& Connolly, 2006; Muyor et al., 2012) wurde in dieser Untersuchung versucht, die Dehnintensität über eine 10-stufige Schmerzskala zu operationalisieren. Die Probanden sollten während des zehnwöchigen Dehntrainings in einem Aktivitätsprotokoll notieren, wie intensiv sie die Dehnungen durchgeführt haben. Allerdings lässt sich nicht ausschlieBen, dass die Probanden mit unterschiedlichen Intensitäten gedehnt haben. Insbesondere ist vorstellbar, dass sich die jüngere Versuchsgruppe, aufgrund einer höheren Leistungsbereitschaft, intensiver gedehnt hat als die ältere Versuchsgruppe. Dies gilt neben dem Dehntraining auch für die Datenaufnahme bei Pre- und Post-Test.

Das Dehnprogramm sollte von den Probanden dreimal in der Woche durchgeführt werden. Davon sollte das Training im Idealfall zweimal pro Woche unter Aufsicht und einmal zu Hause durchgeführt werden. Die Probanden hatten die Aufgabe während des Interventionszeitraumes ein Aktivitätsprotokoll zu führen, indem sie den Tag und die Uhrzeit ihres durchgeführten Dehnprogrammes notieren sollten. Dieses Trainingstagebuch wurde im Anschluss an die Untersuchung zusätzlich herangezogen, um die Compliance der Probanden zu überprüfen. Bei der Auswertung der Trainingstagebücher war man letztendlich auf die Richtigkeit der Angaben seitens der Probanden angewiesen. Bei allen Probanden waren die Aufzeichnungen im Trainingstagebuch plausibel und nachvollziehbar. Allerdings kann nicht ausgeschlossen werden, dass ein Proband sich mal nicht gedehnt hat, es aber dennoch im Aktivitätsprotokoll notierte. Aufgrund der langzeitigen Interventionsphase ließ sich das Problem der Compliance aber nicht anders lösen. Zudem kam es vor, dass einzelne Probanden über mehrere Tage im Interventionszeitraum im Urlaub waren. Diese Proban- 
den führten das vorgegebene Dehntraining dann in dieser Zeit ohne Aufsicht weiter und notierten die Durchführung im Aktivitätsprotokoll.

\subsection{Zusammenfassung}

Mit der empirischen Untersuchung wurden Anpassungserscheinungen durch ein zehnwöchiges Dehntraining zwischen jüngeren und älteren Erwachsenen verglichen. Das Hauptergebnis ist, dass die Adaptionen in der jüngeren Versuchsgruppe höher oder ähnlich waren als in der älteren Versuchsgruppe. Es zeigten sich keine signifikanten altersspezifischen Unterschiede.

Bisher existierten keine Untersuchungen, die biomechanische und neurophysiologische Parameter durch ein langzeitiges Dehntraining zwischen jungen und alten Probanden verglichen. Zusammenfassend konnte gezeigt werden, dass sich das Lebensalter negativ auf die maximale Bewegungsreichweite, die maximale Dehnungsspannung, die passive-elastische Stiffness und die passive-elastische Energie der ischiocruralen Muskulatur auswirkt. Die Anpassungserscheinungen durch ein zehnwöchiges Dehntraining waren in beiden Altersgruppen hinsichtlich des Erreichens einer maximalen Beweglichkeit ähnlich. Darüber hinaus zeigten die jüngeren Erwachsenen hinsichtlich der maximalen Dehnungsspannung sowie der passiven-elastischen Energie nicht signifikant größere prozentuale Veränderungen im Vergleich zu den älteren Erwachsenen. Es kann davon ausgegangen werden, dass ältere Personen genauso von einem regelmäßigen Dehntraining profitieren, wie jüngere Personen. Des Weiteren wurde altersspezifisch untersucht, ob der EMG-Onset einen Einfluss auf die maximale Beweglichkeit hat. Der absolute EMG-Onset war bei beiden Altersgruppen ähnlich und blieb über den Interventionszeitraum nahezu unverändert. Der relative EMG-Onset reduzierte sich in beiden Altersgruppen nicht signifikant und trat zu Pre- und Post-Test bei der alten Altersgruppe zu einem späteren Zeitpunkt auf. Da der relative EMG-Onset abhängig von der maximalen Beweglichkeit ist und die junge Altersgruppe zu allen Messzeitpunkten eine höhere Beweglichkeit erreichte, resultiert dies in einem früheren relativen EMG-Onset der jungen Altersgruppe. 
Da beide Probandengruppen als Freizeitsportler definiert wurden, ohne offensichtliche Einschränkungen der Beweglichkeit, sind diese Ergebnisse zunächst nicht auf die Allgemeinbevölkerung zu übertragen. Zukünftige Studien sollten Anpassungserscheinungen an älteren Personen ohne sportlichen Hintergrund und weniger guten Ausgangsbedingungen untersuchen. Außerdem muss bei der Erhebung muskelelastischer Parameter auf eine einheitliche Begriffsdefin ition geachtet werden. Soll beispielsweise die Stiffness gemessen werden, so sollte diese möglichst nah an der individuellen maximalen Beweglichkeit abgetragen werden. Des Weiteren gilt es zu klären, warum bei manchen Probanden während einer Muskeldehnung ein EMG-Onset ausbleibt. 


\section{Schlussfolgerung und Ausblick}

Mit der hier vorliegenden Arbeit wurde das Ziel verfolgt, Anpassungserscheinungen durch ein Dehntraining altersspezifisch zu vergleichen. Hierfür wurde zunächst eine systematische Literaturrecherche mit anschließender metaanalytischer Betrachtung durchgeführt. Des Weiteren wurden erstmals bei älteren Personen, neben der Anpassung der Beweglichkeit, weitere biomechanische und neurophysiologische Eigenschaften der Muskulatur nach einer langzeitigen Trainingsintervention empirisch untersucht und mit einer jüngeren Altersgruppe verglichen. Aus den Ergebnissen der hier durchgeführten Untersuchungen lassen sich für die Planung und Durchführung eines Dehntrainings für ältere Personen folgende Konsequenzen ableiten:

- Im Rahmen der Metaanalyse konnte gezeigt werden, dass sowohl jüngere als auch ältere Erwachsene nach einem langzeitigen Dehntraining von mindestens zwei Wochen Dauer eine große Effektstärke bezüglich des Erreichens der maximalen Beweglichkeit erlangten. In der empirischen Untersuchung zeigten beide Altersgruppen eine ähnliche prozentuale Veränderung der maximalen Beweglichkeit nach einem zehnwöchigen Dehntraining. Sowohl in der Metaanalyse als auch in der empirischen Untersuchung zeigten sich keine altersspezifischen Unterschiede hinsichtlich der Veränderung der maximalen Beweglichkeit. Es konnte somit festgestellt werden, dass ältere Personen in gleichem Maße von einem langzeitigen Dehntraining profitieren wie jüngere Personen.

- Ein Dehntraining lässt sich neben unterschiedlichen Dehnmethoden auch in unterschiedliche Dehnformen wie Eigen- und Fremddehnung unterteilen. Die Ergebnisse der Metaanalyse konnten zeigen, dass jüngere Personen sowohl mit der Eigen- als auch mit der Fremddehnung eine große Effektstärke bezüglich der maximalen Beweglichkeit erreichten. Ältere Personen hingegen erreichten mit einer Eigendehnung eine große Effektstärke und mit einer Fremddehnung eine mittlere Effektstärke. Es 
konnte somit festgestellt werden, dass ältere Personen eher von einer Eigendehnung profitieren.

- Die Dehnintensität ist stark abhängig vom individuellen subjektiven Schmerzempfinden und der damit einhergehenden Schmerztoleranzgrenze. Aus diesem Grund ist die Intensität einer Muskeldehnung nur schwierig zu operationalisieren. Im Rahmen der Metaanalyse wurde in $32.5 \%$ der untersuchten Primärstudien die Intensität als maximal und zu $45.8 \%$ als submaximal angegeben. Bei $21.7 \%$ der untersuchten Studien wurde keine Intensitätsangabe gemacht. In keiner der untersuchten Studien wurde versucht, die Dehnintensität über eine Intensitäts- oder Schmerzskala zu quantifizieren. Im Hinblick auf die in den Primärstudien angegebenen Intensitätsangaben erreichten jüngere Erwachsene sowohl mit einer maximalen als auch mit einer submaximalen Dehnintensität eine große Effektstärke bezüglich des Erreichens der maximalen Beweglichkeit. Ältere Erwachsene erreichten bei einer maximalen Dehnintensität eine niedrige Effektstärke und bei einer submaximalen Dehnintensität eine große Effektstärke. Es konnte somit festgestellt werden, dass ältere Erwachsene eher von einer submaximalen Dehnintensität profitieren.

- Bezüglich bisheriger Empfehlungen zur Gestaltung eines Dehntrainings wurde im Rahmen der Metaanalyse die Dauer einer Muskeldehnung sowie die Dauer eines Dehntrainings altersspezifisch untersucht. Im Hinblick auf die Dauer einer Muskeldehnung, bezogen auf die passivstatische Dehnmethode, erreichten die älteren Erwachsenen bei einer Muskeldehnung kleiner/gleich 30 Sekunden eine mittlere Effektstärke und bei einer Muskeldehnung länger als 30 Sekunden eine große Effektstärke bezüglich des Erreichens der maximalen Beweglichkeit. Jüngere Erwachsene erreichten sowohl bei kleiner/gleich 30 Sekunden als auch bei einer längeren Dehndauer von über 30 Sekunden große Effektstärken. Bezüglich der Dauer eines Dehntrainings wurde festgestellt, dass ältere Erwachsene bei einem Dehntraining von kleiner/gleich vier 
Wochen eine mittlere Effektstärke und bei einem Dehntraining von mehr als vier Wochen eine große Effektstärke erreichten. Jüngere Erwachsene erreichten sowohl bei einem Dehntraining von kleiner/gleich vier Wochen als auch bei länger als vier Wochen Dauer eine große Effektstärke. Es konnte somit festgestellt werden, dass bei älteren Erwachsenen die Dauer eines Dehntrainings länger als vier Wochen betragen sollte. Des Weiteren profitieren ältere Erwachsene eher von einer längeren Dehndauer von über 30 Sekunden.

- Ergänzend zu dem vorherigen Punkt wurde weiterhin die Trainingshäufigkeit im Rahmen der Metaanalyse altersspezifisch untersucht. Aus den untersuchten Primärstudien wurde ersichtlich, dass sowohl die älteren als auch die jüngeren Erwachsenen nach einem langzeitigen Dehntraining große Effektstärken erreichten. Allerdings war die Trainingshäufigkeit bei den älteren wesentlich höher im Vergleich zu den jüngeren Erwachsenen. Wie im vorherigen Punkt bereits angedeutet benötigen ältere Erwachsene mehr Trainingseinheiten um auf eine ähnlich große Effektstärke bezüglich des Erreichens der maximalen Beweglichkeit zu kommen als jüngere Erwachsene.

Die Verbesserung der maximalen Beweglichkeit nach einem langzeitigen Dehntraining lässt sich sowohl bei älteren als auch bei jüngeren Erwachsenen nach den Ergebnissen der empirischen Untersuchung eher auf eine Anpassung des subjektiven Schmerzempfindens zurückführen. Es konnte nicht festgestellt werden, dass die Muskelelastizität in Form der Stiffness einen signifikanten Einfluss auf die Verbesserung der Beweglichkeit hatte. Des Weiteren konnte nicht festgestellt werden, dass eine neurophysiologische Reflexantwort der Muskulatur während einer Muskeldehnung die Veränderung der maximalen Beweglichkeit durch ein langzeitiges Dehntraining beeinflusst.

Bei Studien am Menschen werden funktionelle Eigenschaften der Muskeln von außen erhoben, aus denen dann auf anatomisch-strukturelle Veränderungen geschlossen werden kann (Klee, 2003, S. 2). In einer Übersichtsarbeit be- 
schreiben Weppler und Magnusson (2010) unterschiedliche Theorien, die zu einer Verbesserung der Beweglichkeit aufgrund anatomisch-struktureller Veränderungen führen könnten. Aufgeführt werden plastische und viskoelastische Veränderungen, Vermehrung der Anzahl an Sarkomeren oder eine neuromuskuläre Entspannung. Wie in der hier vorliegenden Arbeit gehen Weppler und Magnusson (2010) davon aus, dass ein Anstieg der maximalen Beweglichkeit nach einem Dehntraining nicht auf anatomisch-strukturelle Veränderungen, sondern eher auf eine Anpassung der Schmerzwahrnehmung zurückführen ist. $\mathrm{Zu}$ diesem Ergebnis kommen auch Marshall und Siegler (2014) sowie Magnusson et al. (1996).

Die hier vorliegenden Ergebnisse sollten als vorläufige Erkenntnisse betrachtet werden. Es sind weitere Untersuchungen mit älteren Erwachsenen nötig, um die hier gezeigten Ergebnisse zu verifizieren. Mit der Metaanalyse wurde der aktuelle Forschungsstand bezüglich eines langzeitigen Dehntrainings bei jüngeren und älteren Erwachsenen dargestellt. Insbesondere im Hinblick auf ältere Erwachsene zeigte sich ein Forschungsdefizit besonders deutlich, da zu wenige empirische Untersuchungen existieren, die Anpassungserscheinungen durch ein langzeitiges Dehntraining über das Maß der maximalen Beweglichkeit hinaus untersuchten. Mit der hier vorliegenden Untersuchung wurde der erste Schritt unternommen, neben der Beweglichkeit weitere biomechanische und neurophysiologische Anpassungserscheinungen durch ein langzeitiges Dehntraining bei älteren Erwachsenen zu erheben. Zukünftige Studien sollten sich vermehrt mit langzeitigen Dehnungsprogrammen bei älteren Personen beschäftigen, um die hier gewonnen Erkenntnisse auszubauen. Interessant wäre zum Beispiel, altersspezifisch zu klären, wie stabil Anpassungserscheinungen nach einem Dehntraining über die Zeit bleiben. 


\section{Literaturverzeichnis}

Abellaneda, S., Guissard, N., \& Duchateau, J. (2009). The relative lengthening of the myotendinous structures in the medial gastrocnemius during passive stretching differs among individuals. Journal of Applied Physiology, 106(1), 169-177.

Allander, E., Björnsson, O.J., Ólafsson, Ó., Sigfússon, N., \& Thorsteinsson, J. (1974). Normal range of joint movements in shoulder, hip, wrist and thumb with special reference to side. A comparison between two populations. International Journal of Epidemiology, 3(3), 253-261.

doi: $10.1093 / \mathrm{ije} / 3.3 .253$

Alter, M. J. (2004). Science of flexibility (3. Aufl.). Champaign, IL: Human Kinetics.

Apostolopoulos, N., Metsios, G.S., Flouris, A.D., Koutedakis, Y., \& Wyon, M.A. (2015). The relevance of stretch intensity and position. A systematic review. Frontiers in Psychology, 6, 1128.

doi: $10.3389 /$ fpsyg.2015.01128

Ayala, F., \& Baranda, A.P.S. (2010). Effect of 3 different active stretch durations on hip flexion range of motion. Journal of Strength and Conditioning Research, 24(2), 430-436.

Ayala, F., Sainz de Baranda, P., De Ste Croix, M., \& Santonja, F. (2013). Comparison of active stretching technique in males with normal and limited hamstring flexibility. Physical Therapy in Sport, 14(2), 98-104.

Baltes, P.B., \& Baltes, M.B. (1994). Gerontologie. Begriff, Herausforderung und Brennpunkte. In P.B. Baltes, J. Mittelstraß, \& U.M. Staudinger (Hrsg.), Alter und Altern. Ein interdisziplinärer Studientext zur Gerontologie (S. 1-34). Berlin: De Gruyter.

Baluktsian, S. (2017). Bewertung von Dehntrainingsstudien im Rahmen eines systematischen Reviews. Unveröffentlichte Bachelorarbeit, Universität des Saarlandes.

Bandy, W.D., \& Irion, J.M. (1994). The effect of time on static stretch on the flexibility of the hamstring muscles. Physical Therapy, 74(9), 842-845. 
Bandy, W.D., Irion, J.M., \& Briggler, M. (1997). The effect of time and frequency of static stretching on flexibility of the hamstring muscles. Physical Therapy, 77(10), 1090-1096.

Batista, L.H., Vilar, A.C., Ferreira de Almeida, J.J., Rebelatto, J.R., \& Salvini, T.F. (2009). Active stretching improves flexibility, joint torque, and functional mobility in older women. American Journal of Physical Medicine \& Rehabilitation, 88(10), 815-822.

Beck, J. (2006). Datenbank zur motorischen Leistungsfähigkeit (SPODAT II). Bewegungstherapie und Gesundheitssport, 22, 251-252.

Beckers, D. (2013). Techniken. In M. Buck, D. Beckers, \& S.S. Adler (Hrsg.), PNF in der Praxis (7., vollst. überab. Aufl., S. 35-58). Berlin, Heidelberg: Springer.

Bertolasi, L., Grandis, D., Bongiovanni, L.G., Zanette, G.P., \& Gasperini, M. (1993). The influence of muscular lengthening on cramps. Annals of Neurology, 33(2), 176-180.

Bianco, P., Mártonfalvi, Z., Naftz, K., Kőszegi, D., \& Kellermayer, M. (2015). Titin domains progressively unfolded by force are homogenously distributed along the molecule. Biophysical Journal, 109(2), 340-345.

Birbaumer, N., \& Schmidt, R. (2003). Biologische Psychologie (5. Aufl.). Berlin, Heidelberg: Springer.

Björklund, M., Hamberg, J., \& Crenshaw, A.G. (2001). Sensory adaptation after a 2-week stretching regimen of the rectus femoris muscle. Archives of Physical Medicine and Rehabilitation, 82(9), 1245-1250.

Blackburn, J.T., Bell, D.R., Norcross, M.F., Hudson, J.D., \& Kimsey, M.H. (2009). Sex comparison of hamstring structural and material properties. Clinical Biomechanics, 24(1), 65-70.

doi: 10.1016/j.clinbiomech.2008.10.001

Blackburn, J.T., Riemann, B.L., Padua, D.A., \& Guskiewicz, K.M. (2004). Sex comparison of extensibility, passive, and active stiffness of the knee flexors. Clinical Biomechanics, 19(1), 36-43.

doi: 10.1016/j.clinbiomech.2003.09.003 
Blazevich, A.J., Cannavan, D., Waugh, C.M., Fath, F., Miller, S.C., \& Kay, A.D. (2012). Neuromuscular factors influencing the maximum stretch limit of the human plantar flexors. Journal of Applied Physiology, 113(9), 1446-1455.

Blazevich, A.J., Cannavan, D., Waugh, C.M., Miller, S.C., Thorlund, J.B., Aagaard, P., \& Kay, A.D. (2014). Range of motion, neuromechanical, and architectural adaptations to plantar flexor stretch training in humans. Journal of Applied Physiology, 117, 452-462.

Bochdansky, T. (1994). Die Messung der Muskulatur mittels oberflächlicher EMG-Ableitung. Möglichkeiten und Grenzen. In L. Zichner, M. Engelhardt, \& J. Freiwald (Hrsg.), Die Muskulatur. Sensibles, integratives und messbares Organ (S. 59-67). Wehr: Ciba-Geigy.

Bohannon, R., Gajdosik, R., \& LeVeau, B.F. (1985). Contribution of pelvic and lower limb motion to increases in the angle of passive straight leg raising. Physical Therapy, 65(4), 474-476.

Borges, M.O., Medeiros, D.M., Minotto, B.B., \& Lima, C.S. (2017). Comparison between static stretching and proprioceptive neuromuscular facilitation on hamstring flexibility. Systematic review and meta-analysis. European Journal of Physiotherapy. doi: 10.1080/21679169.2017.1347708

Bortz, J., \& Döring, N. (2006). Forschungsmethoden und Evaluation für Humanund Sozialwissenschaftler (4. Aufl.). Heidelberg: Springer.

Buckner, S.L., Jenkins, N.D.M., Costa, P.B., Ryan, E.D., Herda, T.J., \& Cramer, J.T. (2015). Comparing passive angle-torque curves recorded simultaneously with a load cell versus an isokinetic dynamometer during dorsiflexion stretch tolerance assessments. Medical Engineering \& Physics, 37(5), 494-498.

Busse, E.W. (1969). Theories of aging. In E.W. Busse \& E. Pfeiffer (Hrsg.), Behavior and adaptation in later life (S. 11-32). Boston: Little Brown.

Butler, D.L., Grood, E.S., Noyes, F.R., \& Zernicke, R.F. (1978). Biomechanics of ligaments and tendons. Exercise and Sport Sciences Reviews, 6, $125-181$. 
Cabido, C., Pessali-Marques, B., Pereira, B., Magalhaes, F., Reis, M., Santos, T., Rodrigues, S., Andrade, A., Peixoto, G., \& Chagas, M. (2016). EMG onset during passive stretching of hamstring. Presentation at $22 n d$ Congress of the European Society of Biomechanics.

Carlson, C., \& Curran, S. (1994). Stretch-based relaxation training. Patient Education and Counseling, 23(1), 5-12.

Carlson, C.R., Collins, F.L., Nitz, A.J., Sturgis, E.T., \& Rogers, J.L. (1990). Muscle stretching as an alternative relaxation training procedure. Journal of Behavior Therapy and Experimental Psychiatry, 21(1), 29-38.

Chagas, M.H., \& Schmidtbleicher, D. (2004). Auswirkungen von Beweglichkeitstraining auf die Bewegungsamplitude, Dehnungsspannung und Dehngrenze nach einer Trainings- und Detrainingsperiode. 1. Teil: Untersuchungsmethodik und Untersuchungsergebnisse. Leistungssport, 34(5), 28-32.

Chan, S.P., Hong, Y., \& Robinson, P.D. (2001). Flexibility and passive resistance of the hamstrings of young adults using two different static stretching protocols. Scandinavian Journal of Medicine \& Science in Sports, 11, 81-86.

Chesworth, B.M., \& Vandervoort, A.A. (1989). Age and passive ankle stiffness in healthy women. Physical Therapy, 69(3), 217-224.

Chleboun, G. (2005). Muscle structure and function. In P. Levangie \& C. Norkin (Hrsg.), Joint structure and function. A comprehensive analysis (4. Aufl., S. 113-139). Philadelphia: F.A. Davis Co.

Chodzko-Zajko, W.J., Proctor, D.N., Fiatarone Singh, M.A., Minson, C.T., Nigg, C.R., Salem, G.J., \& Skinner, J.S. (2009). American College of Sports Medicine position stand. Exercise and physical activity for older adults. Medicine \& Science in Sports \& Exercise, 41(7), 1510-1530.

Ciena, A.P., Luques, I.U., Dias, F.J., Yokomizo de Almeida, S.R., lyomasa, M.M., \& Watanabe, I.-S. (2010). Ultrastructure of the myotendinous junction of the medial pterygoid muscle of adult and aged wistar rats. Micron, 41(8), 1011-1014. 
Cipriani, D., Abel, B., \& Pirrwitz, D. (2003). A comparison of two stretching protocols on hip range of motion. Implications for total daily stretch duration. Journal of Strength and Conditioning Research, 17(2), 274-278.

Cipriani, D.J., Terry, M.E., Haines, M.A., Tabibnia, A.P., \& Lyssanova, O. (2012). Effect of stretch frequency and sex on the rate of gain and rate of loss in muscle flexibility during a hamstring-stretching program. A randomized single-blind longitudinal study. Journal of Strength and Conditioning Research, 26(8), 2119-2129.

Cohen, J. (1988). Statistical power analysis for the behavioral sciences. Hillsdale: Erlbaum.

Cohen, J. (1992). A power primer. Psychological Bulletin, 112(1), 155-159.

Covert, C.A., Alexander, M.P., Petronis, J.J., \& Davis, D.S. (2010). Comparison of ballistic and static stretching on hamstring muscle length using an equal stretching dose. Journal of Strength and Conditioning Research, 24(11), 3008-3014.

Curwin, S. (2005). Joint structure and function. In P.K. Levangie \& C.C. Norkin (Hrsg.), Joint structure and function. A comprehensive analysis (4. Aufl., S. 69-111). Philadelphia, PA: F.A. Davis Co.

Danner, D., \& Schröder, H. (1994). Biologie des Alterns (Ontogenese und Evolution). In P.B. Baltes, J. Mittelstraß, \& U. Staudinger (Hrsg.), Alter und Altern. Ein interdisziplinärer Studientext zur Gerontologie (S. 95-123). Berlin: De Gruyter.

Davis, D.S., Ashby, P.E., McCale, K.L., McQuain, J.A., \& Wine, J.M. (2005). The effectiveness of 3 stretching techniques on hamstring flexibility using consistent stretching parameters. Journal of Strength and Conditioning Research, 19(1), 27-32.

de Araújo, C. (2004). Flexitest. An innovative flexibility assessment method. Champaign, IL: Human Kinetics.

De Luca, C.J. (1997). The use of surface electromyography in biomechanics. Journal of Applied Biomechanics, 13, 135-163. 
De Luca, C.J. (2006). Electromyography. In J.G. Webster (Hrsg.), Encyclopedia of medical devices and instrumentation (2. Aufl., S. 98-109). Hoboken: John Wiley \& Sons, Inc.

de Mareés, H. (2003). Sportphysiologie. Köln: Sport und Buch Strauss.

de Oliveira Medeiros, H.B., de Araújo, D.S.M.S., \& de Araújo, C.G.S. (2013). Age-related mobility loss is joint-specific. An analysis from 6,000 Flexitest results. AGE, 35(6), 2399-2407. doi: 10.1007/s11357-013-9525-Z

de Weijer, V.C., Gorniak, G.C., \& Shamus, E. (2003). The effect of static stretch and warm-up exercise on hamstring length over the course of 24 hours. Journal of Orthopaedic \& Sports Physical Therapy, 33(12), 727-733. doi: 10.2519/jospt.2003.33.12.727

Decoster, L.C., Cleland, J., Altieri, C., \& Russell, P. (2005). The effects of hamstring stretching on range of motion. A systematic literature review. Journal of Orthopaedic \& Sports Physical Therapy, 35(6), 377-387. doi:10.2519/jospt.2005.35.6.377

Dourte, L.M., Kuntz, A.F., \& Soslowsky, L.J. (2008). Twenty-five years of tendon and ligament research. Journal of Orthopaedic Research, 26(10), 1297-1305.

Eckstein, F., Paulsen, F., \& Tilmann, B. (2010). Organe des Bewegungsapparates. In K. Zilles \& B. Tillmann (Hrsg.), Anatomie (S. 75-307). Heidelberg: Springer.

Edouard, P. (2014). Exercise associated muscle cramps. Discussion on causes, prevention and treatment. Science \& Sports, 29(6), 299-305.

Ensink, F.B., Saur, P.M., Frese, K., Seeger, D., \& Hildebrandt, J. (1996). Lumbar range of motion. Influence of time of day and individual factors on measurements. Spine, 21(11), 1339-1343.

Farage, M.A., Miller, K.W., Ajayi, F., \& Hutchins, D. (2012). Design principles to accommodate older adults. Global Journal of Health Science, 4(2), 2-25.

Faul, F., Erdfelder, E., Buchner, A., \& Lang, A.-G. (2009). Statistical power analyses using $G^{*}$ Power 3.1. Tests for correlation and regression analyses. Behaviour Research Methods, 41(4), 1149-1160. 
Feland, J.B., Myrer, J.W., Schulthies, S.S., Fellingham, G.W., \& Measom, G.W. (2001). The effect of duration of stretching of the hamstring muscle group increasing range of motion in people aged 65 years or older. Physical Therapy, 81(5), 1110-1117.

Felson, D.T., Naimark, A., Anderson, J., Kazis, L., Castelli, W., \& Meenan, R.F. (1987). The prevalence of knee osteoarthritis in the elderly. The Framingham Osteoarthritis Study. Arthritis and Rheumatism, 30(8), 914-918.

Ferreira, G.N.T., Teixeira-Salmela, L.F., \& Guimarães, C.Q. (2007). Gains in flexibility related to measures of muscular performance. Impact of flexibility on muscular performance. Clinical Journal of Sport Medicine, 17(4), 276-281.

Ford, G.S., Mazzone, M.A., \& Taylor, K. (2005). The effect of 4 different durations of static hamstring stretching on passive knee-extension range of motion in healthy subjects. Journal of Sport Rehabilitation, 14(2), 95-107.

Franchi, M., Fini, M., Quaranta, M., Pasquale, V., Raspanti, M., Giavaresi, G., Ottani, G., \& Ruggeri, A. (2007). Crimp morphology in relaxed and stretched rat achilles tendon. Journal of Anatomy, 210(1), 1-7.

Frankel, J.E., Bean, J.F., \& Frontera, W.R. (2006). Exercise in the elderly. Research and clinical practice. Clinics in Geriatric Medicine, 22(2), 239-256.

Freitas, S.R., Vaz, J.R., Bruno, P.M., Valamatos, M.J., Andrade, R.J., \& MilHomens, P. (2015). Are rest intervals between stretching repetitions effective to acutely increase range of motion? International Journal of Sports Physiology and Performance, 10(2), 191-197.

doi: 10.1123/ijspp.2014-0192

Freitas, S.R., Vaz, J.R., Gomes, L., Silvestre, R., Hilário, E., Cordeiro, N., Carnide, F., Pezarat-Correira, P., \& Mil-Homens, P. (2015). A new tool to assess the perception of stretching intensity. Journal of Strength and Conditioning Research, 29(9), 2666-2678. 
Freitas, S.R., Vilarinho, D., Vaz, J.R., Bruno, P.M., Costa, P.B., \& Mil-Homens, P. (2015). Responses to static stretching are dependent on stretch intensity and duration. Clinical Physiology and Functional Imaging, 35(6), 478-484. doi: 10.1111/cpf.12186

Freiwald, J. (2009). Optimales Dehnen. Sport - Prävention - Rehabilitation. Balingen: Spitta.

Freiwald, J., Engelhardt, M., \& Reuter, I. (1998). Neuromuskuläre Dysbalance in Medizin und Sport. Ursachen, Einordnung und Behandlung. In L. Zichner, M. Engelhardt, \& J. Freiwald (Hrsg.), Neuromuskuläre Dysbalancen (S. 165-193). Nürnberg: Novatis Pharma Verlag.

Fröhlich, M., Links, L., \& Pieter, A. (2012). Effekte des Krafttrainings. Eine metaanalytische Betrachtung. Schweizerische Zeitschrift für Sportmedizin und Sporttraumatologie, 60(1), 14-20.

Fröhlich, M., \& Pieter, A. (2009). Cohen's Effektstärken als Mass der Bewertung von praktischer Relevanz. Implikationen für die Praxis. Schweizerische Zeitschrift für Sportmedizin und Sporttraumatologie, 57(4), 139-142.

Gajdosik, R.L. (1991). Effects of static stretching on the maximal length and resistance to passive stretch of short hamstring muscles. The Journal of Orthopaedic and Sports Physical Therapy, 14(6), 250-255.

Gajdosik, R.L. (1997). Influence of age on calf muscle length and passive stiffness variables at different stretch velocities. Isokinetics and Exercise Science, 6(3), 163-174. doi: 10.3233/IES-1997-6301

Gajdosik, R.L. (2001). Passive extensibility of skeletal muscle. Review of the literature with clinical implications. Clinical Biomechanics, 16(2), 87-101.

Gajdosik, R.L., Allred, J.D., Gabbert, H.L., \& Sonsteng, B.A. (2007). A stretching program increases the dynamic passive length and passive resistive properties of the calf muscle-tendon unit of unconditioned younger women. European Journal of Applied Physiology, 99(4), 449-454. 
Gajdosik, R.L., Vander Linden, D.W., McNair, P.J., Williams, A.K., \& Riggin, T.J. (2005). Effects of an eight-week stretching program on the passiveelastic properties and function of the calf muscles of older women. Clinical Biomechanics, 20(9), 973-983.

Gajdosik, R.L., Vander Linden, D.W., \& Williams, A.K. (1999). Influence of age on length and passive elastic stiffness characteristics of the calf muscletendon unit of women. Physical Therapy, 79(9), 827-838.

Gallon, D., Rodacki, A.L.F., Hernandez, S.G., Drabovski, B., Outi, T., Bittencourt, L.R., \& Gomes, A.R.S. (2011). The effects of stretching on the flexibility, muscle performance and functionality of institutionalized older women. Brazilian Journal of Medical and Biological Research, 44(3), 229-235.

Garber, C.E., Blissmer, B., Deschenes, M.R., Franklin, B.A., Lamonte, M.J., Lee, I.-M., Nieman, D.C., \& Swain, D.P. (2011). American College of Sports Medicine position stand. Quantity and quality of exercise for developing and maintaining cardiorespiratory, musculoskeletal, and neuromotor fitness in apparently healthy adults. Guidance for prescribing exercise. Medicine \& Science in Sports \& Exercise, 43(7), 1334-1359.

Ghez, C., \& Gordon, J. (1996). Muskeln und Muskelrezeptoren. In E.R. Kandel, J.H. Schwartz, \& T.M. Jessell (Hrsg.), Neurowissenschaften. Eine Einführung (S. 513-525). Heidelberg: Spektrum.

Glück, S. (2005). Beeinflussung der Beweglichkeit durch unterschiedliche physische und psychische Einwirkungen. Unveröffentlichte Dissertation, Universität des Saarlandes.

Glück, S., Schwarz, M., Hoffmann, U., \& Wydra, G. (2002). Bewegungsreichweite, Zugkraft und Muskelaktivität bei eigen- und fremdregulierter Dehnung. Deutsche Zeitschrift für Sportmedizin, 53(3), 66-71.

Godges, J.J., MacRae, P.G., \& Engelke, K.A. (1993). Effects of exercise on hip range of motion, trunk muscle performance, and gait economy. Physical Therapy, 73(7), 468-477. 
González-Ravé, J.M., Sánchez-Gómez, A., \& Santos-García, D.J. (2012). Efficacy of two different stretch training programs (passive vs. proprioceptive neuromuscular facilitation) on shoulder and hip range of motion in older people. Journal of Strength and Conditioning Research, 26(4), 1045-1051.

Gribble, P.A., Guskiewicz, K.M., Prentice, W.E., \& Shields, E.W. (1999). Effects of static and hold-relax stretching on hamstring range of motion using the FlexAbility LE1000. Journal of Sport Rehabilitation, 8(3), 195-208.

Grimston, S.K., Nigg, B.M., Hanley, D.A., \& Engsberg, J.R. (1993). Differences in ankle joint complex range of motion as a function of age. Foot \& Ankle, 14(4), 215-222.

Grosser, M. (1977). Gelenksbeweglichkeit und Aufwärmeffekt. Leistungssport, $7(1), 38-43$.

Haab, T., De Borger, S., \& Wydra, G. (2017). Altersspezifische Unterschiede nach einem zehnwöchigen Dehntraining. In DGfB (Hrsg.), 10. Jahrestagung der Deutschen Gesellschaft für Biomechanik (DGfB). Abstractband. (S. 168). Hannover.

Haab, T., Martini, J., Baluktsian, S., \& Wydra, G. (2017). Altersspezifische Anpassungen an ein Dehntraining. Eine metaanalytische Betrachtung. German Journal of Exercise and Sport Research. doi: 10.1007/s12662-017-0464-1

Haab, T., Massing, M., \& Wydra, G. (2017). EMG onset after a 10 week stretching intervention. A Comparison between older and younger adults. Journal of Exercise Physiology Online, 20(4), 121-131.

Haab, T., Schmid, S., Sahner, D., Fröhlich, M., Ludwig, O., \& Wydra, G. (2015). Ausgleich einer Longitudinalverschiebung des Beines an einem apparativen Straight-Leg-Raise-Test. In T. Könecke, H. Preuß, \& W.I. Schöllhorn (Hrsg.), Moving Minds - Crossing Boundaries in Sport Science (S. 326). Hamburg: Feldhaus.

Haab, T., \& Wydra, G. (2017). The effect of age on hamstring passive properties after a 10-week stretch training. Journal of Physical Therapy Science, 29(6), 1048-1053. doi: 10.1589/jpts.29.1048 
Hamill, J., \& Knutzen, K. (2009). Biomechanical basis of human movement. Philadelphia: Williams \& Wilkins.

Han, H.-I., Choi, H.-S., \& Shin, W.-S. (2016). Effects of hamstring stretch with pelvic control on pain and work ability in standing workers. Journal of Back and Musculoskeletal Rehabilitation, 29(4), 865-871.

doi: 10.3233/BMR-160703

Hansen, K.A., Weiss, J.A., \& Barton, J.K. (2002). Recruitment of tendon crimp with applied tensile strain. Journal of Biomechanical Engineering, 124(1), 72-77.

Harre, D. (1975). Trainingslehre. Berlin: Sportverlag.

Hawker, G.A., Mian, S., Kendzerska, T., \& French, M. (2011). Measures of adult pain. Visual Analog Scale for Pain (VAS Pain), Numeric Rating Scale for Pain (NRS Pain), McGill Pain Questionnaire (MPQ), Short-Form McGill Pain Questionnaire (SF-MPQ), Chronic Pain Grade Scale (CPGS), Short Form-36 Bodily Pain Scale (SF-36 BPS), and Measure of Intermittent and Constant Osteoarthritis Pain (ICOAP). Arthritis Care \& Research, 63(S11), S240-S252. doi: 10.1002/acr.20543

Hayes, B.T., Harter, R.A., Widrick, J.J., Williams, D.P., Hoffman, M.A., \& HicksLittle, C.A. (2012). Lack of neuromuscular origins of adaptation after a long-term stretching program. Journal of Sport Rehabilitation, 21(2), 99-106.

Hedges, L.V. (1981). Distribution theory for Glass's estimator of effect size and related estimators. Journal of Educational and Behavioral Statistics, 6(2), 107-128.

Hedges, L.V, \& Olkin, I. (1985). Statistical methods for meta-analysis. Orlando: Academic Press.

Hermens, H.J., Freriks, B., Merletti, R., Stegeman, D., Blok, J., Rau, G., Disselhorst-Klug, C., \& Hägg, G. (1999). European recommendations for surface ElectroMyoGraphy results of the SENIAM project. Enschede: Roessingh Research and Development.

Hershey, D. (1984). Must we grow old? Cincinnati: Basal Books. 
Higgins, J., \& Green, S. (Hrsg.). (2008). Cochrane handbook for systematic reviews of interventions. The Cochrane Collaboration.

doi: $10.1002 / 9780470712184$

Hodges, P.W., \& Bui, B.H. (1996). A comparison of computer-based methods for the determination of onset of muscle contraction using electromyography. Electroencephalography and Clinical Neurophysiology, 101(6), 511-519.

Hoge, K.M., Ryan, E.D., Costa, P.B., Herda, T.J., Walter, A.A., Stout, J.R., \& Cramer, J.T. (2010). Gender differences in musculotendinous stiffness and range of motion after an acute bout of stretching. Journal of Strength and Conditioning Research, 24(10), 2618-2626.

Holland, G.J., Tanaka, K., Shigematsu, R., \& Nakagaichi, M. (2002). Flexibility and physical functions of older adults. A review. Journal of Aging and Physical Activity, 10(2), 169-206.

Holt, L., Pelham, T., \& Holt, J. (2008). Flexibility. A concise guide. To conditioning, performance enhancement, injury prevention, and rehabilitation. Totowa: Humana Press.

Hooper, A.C. (1981). Length, diameter and number of ageing skeletal muscle fibres. Gerontology, 27(3), 121-126.

Horowits, R., \& Podolsky, R.J. (1987). The positional stability of thick filaments in activated skeletal muscle depends on sarcomere length. Evidence for the role of titin filaments. The Journal of Cell Biology, 105(5), 2217-2223.

Hung, C.T., \& Mow, V.C. (2012). Biomechanics of articular cartilage. In M. Nordin \& V.H. Frankel (Hrsg.), Basic biomechanics of the musculoskeletal system (4. Aufl., S. 60-101). Philadelphia: Lippincott Williams \& Wilkins.

Hutton, R. (1994). Neuromuskuläre Grundlagen des Stretchings. In P. Komi (Hrsg.), Kraft und Schnellkraft im Sport (S. 41-50). Köln: Deutscher Ärzte Verlag.

Huxley, H. E. (2004). Fifty years of muscle and the sliding filament hypothesis. European Journal of Biochemistry, 271(8), 1403-1415. 
Huxley, H., \& Hanson, J. (1954). Changes in the cross-striations of muscle during contraction and stretch and their structural interpretation. Nature, 173(4412), 973-976.

Jami, L. (1992). Golgi tendon organs in mammalian skeletal muscle. Functional properties and central actions. Physiological Reviews, 72(3), 623-666.

Johns, R.J., \& Wright, V. (1962). Relative importance of various tissues in joint stiffness. Journal of Applied Physiology, 17(5), 824-828.

Johnson, A.W., Mitchell, U.H., Meek, K., \& Feland, J.B. (2014). Hamstring flexibility increases the same with 3 or 9 repetitions of stretching held for a total time of $90 \mathrm{~s}$. Physical Therapy in Sport, 15(2), 101-105.

Johnson, E.G., Bradley, B.D., Witkowski, K.R., \& McKee, R.Y. (2007). Effect of a static calf muscle-tendon unit stretching program on ankle dorsiflexion range of motion of older women. Journal of Geriatric Physical Therapy, 30(2), 49-52.

Kannus, P. (2000). Structure of the tendon connective tissue. Scandinavian Journal of Medicine \& Science in Sports, 10(6), 312-320.

Kellermayer, M.S., Smith, S.B., Granzier, H.L., \& Bustamante, C. (1997). Folding-unfolding transitions in single titin molecules characterized with laser tweezers. Science, 276(5315), 1112-1116.

Kerrigan, D.C., Xenopoulos-Oddsson, A., Sullivan, M.J., Lelas, J.J., \& Riley, P.O. (2003). Effect of a hip flexor-stretching program on gait in the elderly. Archives of Physical Medicine and Rehabilitation, 84(1), 1-6.

Kisner, C., \& Colby, L.A. (2012). Therapeutic Exercise. Foundations and Techniques (6. Aufl.). Philadelphia: F.A. Davis Co.

Kjaer, M. (2004). Role of extracellular matrix in adaptation of tendon and skeletal muscle to mechanical loading. Physiological Reviews, 84(2), 649-698.

Klee, A. (2003). Methoden und Wirkungen des Dehnungstrainings. Die Ruhespannungs-Dehnungskurve. Ihre Erhebung beim M.rectus femoris und ihre Veränderung im Rahmen kurzfristiger Treatments. Schorndorf: Hofmann. 
Klee, A., Jöllenbeck, T., \& Wiemann, K. (2000). Messstation zur Erfassung des Dehnungswiderstandes und der Viskosität der ischiocruralen Muskeln und des M. rectus femoris. Ein Vergleich. In K. Nicol \& K. Peikenkamp (Hrsg.), Apparative Biomechanik - Methodik und Anwendungen: 5. Symposium der dvs-Sektion Biomechanik (S. 299-303). Hamburg: Czwalina.

Klee, A., Jöllenbeck, T., \& Wiemann, K. (2002). The significance of titin filaments to resting tension and posture. In Y. Hong (Hrsg.), International Research in Sports Biomechanic (S. 90-97). London: Routledge.

Klee, A., \& Wiemann, K. (2000). Der Einfluss von Flüssigkeitsverschiebungen auf die Bestimmung muskulärer Dehnungsparameter. Deutsche Zeitschrift für Sportmedizin, 51(6), 205-210.

Klee, A., \& Wiemann, K. (2005). Beweglichkeit / Dehnfähigkeit. Schorndorf: Hofmann.

Knott, M., \& Voss, D.E. (1968). Proprioceptive neuromuscular facilitation. New York: Harper \& Row.

Knudsen, A.B., Larsen, M., Mackey, A.L., Hjort, M., Hansen, K.K., Qvortrup, K., Kiaer, M., \& Krogsgaard, M.R. (2015). The human myotendinous junction. An ultrastructural and 3D analysis study. Scandinavian Journal of Medicine \& Science in Sports, 25(1), e116-e123.

Knudson, D. (2006). The biomechanics of stretching. Journal of Exercise Science \& Physiotherapy, 2, 3-12.

Konrad, A., \& Tilp, M. (2014). Increased range of motion after static stretching is not due to changes in muscle and tendon structures. Clinical Biomechanics, 29(6), 636-642.

Krämer, J., \& Grifka, J. (2005). Orthopädie. Heidelberg: Springer.

Krause, F., Wilke, J., Vogt, L., \& Banzer, W. (2016). Intermuscular force transmission along myofascial chains. A systematic review. Journal of Anatomy, 228(6), 910-918. doi: 10.1111/joa.12464

Kubo, K., Kanehisa, H., \& Fukunaga, T. (2002). Effect of stretching training on the viscoelastic properties of human tendon structures in vivo. Journal of Applied Physiology, 92(2), 595-601. doi: 10.1152/japplphysiol.00658.2001 
Kubo, K., Kanehisa, H., Kawakami, Y., \& Fukunaga, T. (2001). Influence of static stretching on viscoelastic properties of human tendon structures in vivo. Journal of Applied Physiology, 90(2), 520-527.

Kuhlman, K., Lehmann, J., Keating, J., Bolles, S., Braun-Porter, L., \& Pastrick, M. (1993). Cervical range of motion in the elderly. Archives of Physical Medicine and Rehabilitation, 74(10), 1071-1079.

doi: 10.1016/0003-9993(93)90064-H

Landis, J.R., \& Koch, G.G. (1977). The measurement of observer agreement for categorical data. Biometrics, 33(1), 159-174.

LaRoche, D.P., \& Connolly, D.A.J. (2006). Effects of stretching on passive muscle tension and response to eccentric exercise. The American Journal of Sports Medicine, 34(6), 1000-1007.

Latash, M., \& Zatsiorsky, V. (1993). Joint stiffness. Myth or reality? Human Movement Science, 12, 653-692.

Law, M., Stewart, D., Pollock, N., Letts, L., Bosch, J., \& Westmorland, M. (1998). Guidelines for critical review form. Quantitative studies. In McMaster University \& Occupational Therapy Evidence-based Practice Research Group (Hrsg.), Zugriff am 23.06.2017. Verfügbar unter http://srsmcmaster.ca/wp-content/uploads/2015/05/Guidelines-for-Critical-ReviewForm-Quantitative-Studies.pdf

Lehmann-Horn, F. (2010). Motorische Systeme. In R.F. Schmidt, F. Lang, \& M. Heckmann (Hrsg.), Physiologie des Menschen mit Pathophysiologie (31. überarb. Aufl., S. 129-162). Heidelberg: Springer.

Lexell, J. (1995). Human aging, muscle mass, and fiber type composition. The Journals of Gerontology. Series A, 50 Spec No, 11-16.

Lexell, J., Taylor, C.C., \& Sjöström, M. (1988). What is the cause of the ageing atrophy? Total number, size and proportion of different fiber types studied in whole vastus lateralis muscle from 15- to 83-year-old men. Journal of the Neurological Sciences, 84(2-3), 275-294.

Lin, L. (1992). Assay validation using the concordance correlation coefficient. Biometrics, 48(2), 599-604. doi: 10.2307/2532314 
Lindel, K. (2010). Muskeldehnung. Grundlagen, Differenzialdiagnostik, therapeutische Dehnungen, Eigendehnungen. Physiotherapie-Basics (2. Aufl.). Berlin, Heidelberg, New York: Springer Medizin.

Linke, W.A. (2000). Stretching molecular springs. Elasticity of titin filaments in vertebrate striated muscle. Histology and Histopathology, 15(3), 799-811.

Liversage, A.D., Holmes, D., Knight, P.J., Tskhovrebova, L., \& Trinick, J. (2001). Titin and the sarcomere symmetry paradox. Journal of Molecular Biology, 305(3), 401-409.

López-Bedoya, J., Vernetta-Santana, M., Robles-Fuentes, A., \& Ariza-Vargas, L. (2013). Effect of three types of flexibility training on active and passive hip range of motion. The Journal of Sports Medicine and Physical Fitness, 53(3), 304-311.

Lundberg, U., Kadefors, R., Melin, B., Palmerud, G., Hassmén, P., Engström, M., \& Elfsberg Dohns, I. (1994). Psychophysiological stress and EMG activity of the trapezius muscle. International Journal of Behavioral Medicine, 1(4), 354-370.

Magnusson, S.P. (1998). Passive properties of human skeletal muscle during stretch maneuvers. A review. Scandinavian Journal of Medicine \& Science in Sports, 8, 65-77.

Magnusson, S.P., Aagaard, P., \& Nielson, J.J. (2000). Passive energy return after repeated stretches of the hamstring muscle-tendon unit. Medicine \& Science in Sports \& Exercise, 32(6), 1160-1164.

Magnusson, S.P., Simonsen, E.B., Aagaard, P., Boesen, J., Johannsen, F., \& Kjaer, M. (1997). Determinants of musculoskeletal flexibility. Viscoelastic properties, cross-sectional area, EMG and stretch tolerance. Scandinavian Journal of Medicine \& Science in Sports, 7, 195-202.

Magnusson, S.P., Simonsen, E.B., Aagaard, P., Sorensen, H., \& Kjaer, M. (1996). A mechanism for altered flexibility in human skeletal muscle. Journal of Physiology, 497(1), 291-298.

Maher, C.G., Sherrington, C., Herbert, R.D., Moseley, A.M., \& Elkins, M. (2003). Reliability of the PEDro scale for rating quality of randomized controlled trials. Physical Therapy, 83(8), 713-721. 
Mahieu, N.N., Cools, A., Wilde, B., Boon, M., \& Witvrouw, E. (2009). Effect of proprioceptive neuromuscular facilitation stretching on the plantar flexor muscle-tendon tissue properties. Scandinavian Journal of Medicine \& Science in Sports, 19(4), 553-560.

Mahieu, N.N., McNair, P., Muynck, M., Stevens, V., Blanckaert, I., Smits, N., \& Witvrouw, E. (2007). Effect of static and ballistic stretching on the muscletendon tissue properties. Medicine \& Science in Sports \& Exercise, 39(3), 494-501.

Marschall, F. (1999). Wie beeinflussen unterschiedliche Dehnintensitäten kurzfristig die Veränderung der Bewegungsreichweite? Deutsche Zeitschrift für Sportmedizin, 50(1), 5-9.

Marshall, P.W.M., Cashman, A., \& Cheema, B. S. (2011). A randomized controlled trial for the effect of passive stretching on measures of hamstring extensibility, passive stiffness, strength, and stretch tolerance. Journal of Science and Medicine in Sport, 14(6), 535-540.

Marshall, P.W.M., \& Siegler, J.C. (2014). Lower hamstring extensibility in men compared to women is explained by differences in stretch tolerance. BMC Musculoskeletal Disorders, 15(223). doi: 10.1186/1471-2474-15-223

Martin, D., Carl, K., \& Lehnertz, K. (2001). Handbuch Trainingslehre (3. unveränd. Aufl.). Schorndorf: Karl Hofmann.

Martini, J. (2016). Ein systematischer Review zur Durchführung und Auswertung von Dehntrainingsstudien. Unveröffentlichte Bachelorarbeit, Universität des Saarlandes.

Maruyama, K., Kimura, S., Ohashi, K., \& Kuwano, Y. (1981). Connectin, an elastic protein of muscle. Identification of "titin" with connectin. Journal of Biochemistry, 89(3), 701-709.

Maruyama, K., Matsubara, S., Natori, R., Nonomura, Y., \& Kimura, S. (1977). Connectin, an elastic protein of muscle. Characterization and Function. Journal of Biochemistry, 82(2), 317-337.

Matthews, P.B.C (1981). Evolving views on the internal operation and functional role of the muscle spindle. The Journal of Physiology, 320, 1-30. 
Matthews, P.B.C. (1964). Muscle spindles and their motor control. Physiological Reviews, 44(2), 219-288.

Matthews, P.B.C. (1972). Mammalian muscle receptors and their central actions. Baltimore: Williams and Wilkins.

McGraw, L.O., \& Wong, S.P. (1996). Forming inferences about some intraclass correlation coefficients. Psychological Methods, 1(1), 30-46.

McHugh, M.P., Kremenic, I.J., Fox, M.B., \& Gleim, G.W. (1998). The role of mechanical and neural restraints to joint range of motion during passive stretch. Medicine \& Science in Sports \& Exercise, 30(6), 928-932.

McNair, P.J., Hewson, D.J., Dombroski, E., \& Stanley, S.N. (2002). Stiffness and passive peak force changes at the ankle joint. The effect of different joint angular velocities. Clinical Biomechanics, 17(7), 536-540.

Medeiros, D.M., Cini, A., Sbruzzi, G., \& Lima, C.S. (2016). Influence of static stretching on hamstring flexibility in healthy young adults. Systematic review and meta-analysis. Physiotherapy Theory and Practice, 32(6), 438-445. doi: 10.1080/09593985.2016.1204401

Merletti, R., Rainoldi, A., \& Farina, D. (2001). Surface electromyography for noninvasive characterization of muscle. Exercise and Sport Science Reviews, 29(1), 20-25.

Miller, K.C., Stone, M.S., Huxel, K.C., \& Edwards, J.E. (2010). Exerciseassociated muscle cramps. Causes, treatment, and prevention. Sports Health, 2(4), 279-283.

Morini, S., Bassi, A., Cerulli, C., Marinozzi, A., \& Ripani, M. (2004). Hip and ankle joints flexibility in young and elderly people. Effect of physical activity in the elderly. Biology of Sport, 21(4), 25-37.

Muanjai, P., Jones, D.A., Mickevicius, M., Satkunskiene, D., Snieckus, A., Rutkauskaite, R., Mickeviciene, D., \& Kamandulis, S. (2017). The effects of 4 weeks stretching training to the point of pain on flexibility and muscle tendon unit properties. European Journal of Applied Physiology. doi: 10.1007/s00421-017-3666-1 
Muyor, J.M., Lopez-Minarro, P.A., \& Casimiro, A.J. (2012). Effect of stretching program in an industrial workplace on hamstring flexibility and sagittal spinal posture of adult women workers. A randomized controlled trial. Journal of Back and Musculoskeletal Rehabilitation, 25(3), 161-169.

Myers, T.W., Frenk, S., \& Hamilton, N. (1997). The "anatomy trains." Journal of Bodywork and Movement Therapies, 1(2), 91-101.

doi: 10.1016/S1360-8592(97)80010-1

Nahler, B. (1998). Annäherung der anatomischen Drehachse des Hüftgelenks an die mechanische Drehachse einer Meßapparatur zur Messung der Beweglichkeit im Hüftgelenk. Unveröffentlichte Diplomarbeit, Universität des Saarlandes.

Neumann, G. (1998). Zur Begriffsbestimmung muskulärer Dysbalancen. In L. Zichner, M. Engelhardt, \& J. Freiwald (Hrsg.), Neuromuskuläre Dysbalancen (S. 9-23). Nürnberg: Novatis Pharma Verlag.

Nonaka, H., Mita, K., Watakabe, M., Akataki, K., Suzuki, N., Okuwa, T., \& Yabe, K. (2002). Age-related changes in the interactive mobility of the hip and knee joints. A geometrical analysis. Gait \& Posture, 15(3), 236-243.

Nordez, A., McNair, P., Casari, P., \& Cornu, C. (2008). Acute changes in hamstrings musculo-articular dissipative properties induced by cyclic and static stretching. International Journal of Sports Medicine, 29(5), 414-418. doi: $10.1055 / \mathrm{s}-2007-964980$

Nuki, G. (1999). Osteoarthritis. A problem of joint failure. Zeitschrift für Rheumatologie, 58(3), 142-147.

Nürnberger, F. (1998). Lokalisation und Funktion von Rezeptoren im GelenkMuskel-Komplex. In L. Zichner, M. Engelhardt, \& J. Freiwald (Hrsg.), Neuromuskuläre Dysbalancen (S. 25-38). Nürnberg: Novatis Pharma Verlag.

Olivier, N., Marschall, F., \& Büsch, D. (2008). Grundlagen der Trainingswissenschaft und -lehre. Schorndorf: Hofmann.

Overend, T.J., Cunningham, D.A., Paterson, D.H., \& Lefcoe, M.S. (1992). Thigh composition in young and elderly men determined by computed tomography. Clinical Physiology, 12(6), 629-640. 
Paul, J., Balakrishnan, P., \& Izham, M. (2014). Comparative effect of static and dynamic stretching exercise to improve flexibility of hamstring muscles among non athletes. International Journal of Physiotherapy, 1(4), 195-199.

Peixoto, G.H., Andrade, A.G., Menzel, H.J.K., Araújo, S.R.S., Pertence, A.E.M., \& Chagas, M.H. (2015). Viscoelastic stress relaxation in the hamstrings before and after a 10-week stretching program. Muscle \& Nerve, 51(5), 761-764. doi: 10.1002/mus.24593

Pereira, D., Peleteiro, B., Araújo, J., Branco, J., Santos, R., \& Ramos, E. (2011). The effect of osteoarthritis definition on prevalence and incidence estimates. A systematic review. Osteoarthritis and Cartilage, 19(11), 1270-1285. doi: 10.1016/j.joca.2011.08.009

Pfeifer, K., Vogt, L., \& Banzer, W. (2003). Kinesiologische Elektromyographie (EMG). Deutsche Zeitschrift für Sportmedizin, 54(11), 331-332.

Politou, A.S., Thomas, D.J., \& Pastore, A. (1995). The folding and stability of titin immunoglobulin-like modules, with implications for the mechanism of elasticity. Biophysical Journal, 69(6), 2601-2610.

Pollock, M.L., Gaesser, G.A., Butcher, J.D., Després, J.P., Dishman, R.K., Franklin, B.A., \& Garber, C.E. (1998). ACSM Position Stand. The recommended quantity and quality of exercise for developing and maintaining cardiorespiratory and muscular fitness, and flexibility in healthy adults. Medicine \& Science in Sports \& Exercise, 30(6), 975-991.

Pospeschill, M. (2013). Empirische Methoden in der Psychologie. München: Ernst Reinhardt, GmbH \& Co KG.

Purslow, P.P. (1989). Strain-induced reorientation of an intramuscular connective tissue network. Implications for passive muscle elasticity. Journal of Biomechanics, 22(1), 21-31.

doi: 10.1016/0021-9290(89)90181-4

Purslow, P.P., \& Delage, J.-P. (2014). Allgemeine Anatomie der Muskelfaszie. In R. Schleip, T. Findley, L. Chaitow, \& P. Huijing (Hrsg.), Lehrbuch Faszien. Grundlagen, Forschung, Behandlung (S. 4-8). München: Urban \& Fischer. 
Rancour, J., Holmes, C.F., \& Cipriani, D.J. (2009). The effects of intermittent stretching following a 4-week static stretching protocol. A rondomized trial. Journal of Strength and Conditioning Research, 23(8), 2217-2222.

Regnier, M., \& Cheng, Y. (2016). Finally, we can relax. A new generation of muscle models that incorporate sarcomere compliance. Biophysical Journal, 110(3), 521-522.

Reid, D.A., \& McNair, P.J. (2004). Passive force, angle, and stiffness changes after stretching of hamstring muscles. Medicine \& Science in Sports \& Exercise, 36(11), 1944-1948.

Rowlands, A.V, Marginson, V.F., \& Lee, J. (2003). Chronic flexibility gains. Effect of isometric contraction duration during proprioceptive neuromuscular facilitation stretching techniques. Research Quarterly for Exercise and Sport, 74(1), 47-51.

Rustenbach, S. (2003). Metaanalyse. Eine anwendungsorientierte Einführung. Bern: Hans Huber.

Ryan, E.D., Herda, T.J., Costa, P.B., Herda, A.A., \& Cramer, J.T. (2014). Acute effects of passive stretching of the plantarflexor muscles on neuromuscular function. The influence of age. Age, 36(4), 9672.

doi: $10.1007 / \mathrm{s} 11357-014-9672-x$

Ryan, E.D., Herda, T.J., Costa, P.B., Walter, A.A., \& Cramer, J.T. (2012). Dynamics of viscoelastic creep during repeated stretches. Scandinavian Journal of Medicine \& Science in Sports, 22(2), 179-184.

doi: $10.1111 / \mathrm{j} .1600-0838.2010 .01285 . x$

Sainz de Baranda, P., \& Ayala, F. (2010). Chronic flexibility improvement after 12 week of stretching program utilizing the ACSM recommendations. Hamstring flexibility. International Journal of Sports Medicine, 31(6), 389-396.

Schiebler, T.H., \& Korf, H.-W. (2007). Anatomie. Histologie, Entwicklungsgeschichte, makroskopische und mikroskopische Anatomie, Topographie (10. Aufl.). Darmstadt: Steinkopff. 
Schleip, R. (2003). Fascial plasticity? A new neurobiological explanation. Part 1. Journal of Bodywork and Movement Therapies, 7(1), 11-19. doi: 10.1016/S1360-8592(02)00067-0

Schnabel, G. (2005). Training der Beweglichkeit. In G. Schnabel, D. Harre, J. Krug, \& A. Borde (Hrsg.), Trainingswissenschaft. Leistung, Training, Wettkampf (S. 280-291). Berlin: Sportverlag Berlin.

Schneider, S., Schmitt, H., Zalewski, M., \& Gantz, S. (2011). Dehnst Du noch oder grübelst Du schon? Aktuelle Daten zu Akzeptanz und Verbreitung von Stretching im Leistungssport. Deutsche Zeitschrift für Sportmedizin, 62(3), 75-78.

Schomacher, J. (2005). Biomechanik der Körperstrukturen. In A. Hüter-Becker \& M. Dölken (Hrsg.), Biomechanik, Bewegungslehre, Leistungsphysiologie, Trainingslehre (2. Aufl., S. 67-127). Stuttgart: Thieme.

Schomburg, E. (1998). Spinale Mechanismen zur Steuerung neuromuskulärer Balance. In L. Zichner, M. Engelhardt, \& J. Freiwald (Hrsg.), Neuromuskuläre Dysbalancen (S. 39-57). Nürnberg: Novatis Pharma Verlag.

Schönthaler, S.R., Ohlendorf, K., Ott, H., Meyer, T., Kindermann, W., \& Schmidtbleicher, D. (1998). Biomechanische und neurophysiologische Parameter zur Erfassung der Dehnbarkeit von Muskel-Sehnen-Einheiten. Deutsche Zeitschrift für Sportmedizin, 49(7/8), 223-230.

Schönthaler, S.R., \& Ott, H. (1994). Auswirkungen verschiedener Dehnmethoden auf die maximale Bewegungsreichweite und die Dehnungsspannung. Messung an der ischiocruralen Muskulatur mit einem computergesteuerten isokinetischen Meßsystem. Unveröffentlichte Diplomarbeit, Universität des Saarlandes.

Schönthaler, S.R., Ott, H., Ohlendorf, K., Nahler, B., Schmidtbleicher, D., \& Kindermann, W. (1998). Determination of the rotation axis of the hip joint flexibility measurement of the hamstrings. International Journal of Sports Medicine, 19(Suppl. 1), S61.

Schönthaler, S.R., \& Ohlendorf, K. (2002). Biomechanische und neurophysiologische Veränderungen nach ein- und mehrfach seriellem passivstatischem Beweglichkeitstraining (1. Aufl). Köln: Sport und Buch Strauss. 
Seifert, T.L. (1991). Determining effect sizes in various experimental designs. Educational and Psychological Measurement, 51(2), 341-347.

Silbernagl, S., \& Despopoulos, A. (2007). Taschenatlas Physiologie. Stuttgart: Thieme.

Smith, C.A. (1994). The warm-up procedure. To stretch or not to stretch. A brief review. The Journal of Orthopaedic and Sports Physical Therapy, 19(1), 12-17.

Smith, J., \& Walker, J.(1983). Knee and elbow range of motion in healthy older individuals. Physical \& Occupational Therapy in Geriatrics, 2(4), 31-38. doi: 10.1080/J148V02N04_04

Sobolewski, E.J., Ryan, E.D., Thompson, B.J., McHugh, M.P., \& Conchola, E.C. (2014). The influence of age on the viscoelastic stretch response. Journal of Strength and Conditioning Research, 28(4), 1106-1112. doi: $10.1519 /$ JSC. 0000000000000326

Spirduso, W.W., Francis, K.L., \& MacRea, P.G. (2005). Physical dimensions of aging. Champaign, IL: Human Kinetics.

Spitzer, R.L., Cohen, J., Fleiss, J.L., \& Endicott, J. (1967). Quantification of agreement in psychiatric diagnosis. Archives of General Psychiatry, 17(1), 83-87.

Stathokostas, L., McDonald, M.W., Little, R.M.D., \& Paterson, D.H. (2013). Flexibility of older adults aged 55-86 years and the influence of physical activity. Journal of Aging Research, 2013, 743843. doi: $10.1155 / 2013 / 743843$

Stock, W.A. (1994). Systematic coding for research synthesis. In H.M. Cooper \& L.V Hedges (Hrsg.), The Handbook of Research Synthesis (S. 125-138). New York: Russell Sage Foundation.

Sun, Y., Stürmer, T., Günther, K., \& Brenner, H. (1997). Inzidenz und Prävalenz der Cox- und Gonarthrose in der Allgemeinbevölkerung. Zeitschrift für Orthopädie und Unfallchirurgie, 135(3), 184-192. doi: $10.1055 / \mathrm{s}-2008-1039578$

Thienes, G. (2000). Beweglichkeitstraining. Grundlagen, Methoden, Leistungssteuerung, Übungen. München: BLV. 
Toft, E., Espersen, G.T., Kålund, S., Sinkjaer, T., \& Hornemann, B.C. (1989). Passive tension of the ankle before and after stretching. The American Journal of Sports Medicine, 17(4), 489-494.

Tricoli, V. (2011). Skeletal muscle physiology. In M. Cardinale, R. Newton, \& K. Nosaka (Hrsg.), Strength and Conditioning. Biological Principles and Practical Applications (S. 3-16). Chichester: Wiley-Blackwell.

Tskhovrebova, L., \& Trinick, J. (2010). Roles of titin in the structure and elasticity of the sarcomere. Journal of Biomedicine \& Biotechnology, 2010, 612482. doi: $10.1155 / 2010 / 612482$

Tskhovrebova, L., Trinick, J., Sleep, J.A., \& Simmons, R.M. (1997). Elasticity and unfolding of single molecules of the giant muscle protein titin. Nature, 387(6630), 308-312.

Van Tulder, M., Malmivaara, A., Esmail, R., \& Koes, B. (2000). Exercise therapy for low back pain. Spine, 25(21), 2784-2796.

Wang, K., McCarter, R., Wright, J., Beverly, J., \& Ramirez-Mitchell, R. (1991). Regulation of skeletal muscle stiffness and elasticity by titin isoforms. A test of the segmental extension model of resting tension. Proceedings of the National Academy of Sciences of the United States of America, 88(16), 7101-7105.

Wang, K., McCarter, R., Wright, J., Beverly, J., \& Ramirez-Mitchell, R. (1993). Viscoelasticity of the sarcomere matrix of skeletal muscles. The titinmyosin composite filament is a dual-stage molecular spring. Biophysical Journal, 64(4), 1161-1177. doi: 10.1016/S0006-3495(93)81482-6

Warren, P.J., Hardiman, P.J., \& Woolf, V.J. (1992). Limb exsanguination. II. The leg: effect of angle of elevation. Annals of the Royal College of Surgeons of England, 74(5), 323-325.

Watt, J.R., Jackson, K., Franz, J.R., Dicharry, J., Evans, J., \& Kerrigan, D.C. (2011). Effect of a supervised hip flexor stretching program on gait in elderly individuals. $P M \& R, 3(4), 324-329$.

Weineck, J. (2007). Optimales Training. Leistungsphysiologische Trainingslehre unter besonderer Berücksichtigung des Kindes- und Jugendtrainings (15. Aufl.). Balingen: Spitta Verlag. 
Weppler, C.H., \& Magnusson, S.P. (2010). Increasing muscle extensibility. A matter of increasing length or modifying sensation? Physical Therapy, 90(3), 438-449.

Whiting, A., Wardale, J., \& Trinick, J. (1989). Does titin regulate the length of muscle thick filaments? Journal of Molecular Biology, 205(1), 263-268.

Wiemann, K. (1991). Beeinflussung muskulärer Parameter durch ein zehnwöchiges Dehnungstraining. Sportwissenschaft, 21(3), 295-306.

Wiemann, K. (1994). Beeinflussung muskulärer Parameter durch unterschiedliche Dehnverfahren. In M. Hostert \& H.-U. Nepper (Hrsg.), Dehnen und Mobilisieren (S. 40-71). Öhringen: Speh-Druck.

Wiemann, K., Klee, A., \& Startmann, M. (1998). Filamentäre Quellen der Muskel-Ruhespannung und die Behandlung muskulärer Dysbalancen. Deutsche Zeitschrift für Sportmedizin, 49(4), 111-118.

Wilkening, F., \& Wilkening, K. (1985). Kriterien von Versuchsplänen (Studieneinheit Versuchsplanung, VERS/4). In W. F. Kugemann \& W. Toman (Hrsg.), Studienmaterielien FIM-Psychologie. Erlangen, Tübingen: DIFF, Universität Erlangen-Nürnberg.

Witvrouw, E., Mahieu, N., Danneels, L., \& McNair, P. (2004). Stretching and injury prevention. An obscure relationship. Sports Medicine, 34(7), 443-449.

Wortman, P.M. (1994). Judging research quality. In H.M. Cooper \& L.V Hedges (Hrsg.), The Handbook of Research Synthesis (S. 97-109). New York: Russell Sage Foundation.

Wydra, G. (2008). Die Trainierbarkeit der Beweglichkeit in der Lebensspanne. Reanalyse vorliegender Studien unter Alternsgesichtspunkten. Pt Zeitschrift für Physiotherapeuten, 60(12), 1312-1331.

Wydra, G. (2009). Entwicklung der Beweglichkeit. In J. Baur, K. Bös, A. Conzelmann, \& R. Singer (Hrsg.), Handbuch Motorische Entwicklung (S. 187-195). Schorndorf: Hofmann.

Wydra, G., Bös, K., \& Karisch, G. (1991). Zur Effektivität verschiedener Dehntechniken. Deutsche Zeitschrift für Sportmedizin, 42(9), 386-400. 
Wydra, G., Glück, S., \& Roemer, K. (1999). Kurzfristige Effekte verschiedener singulärer Muskeldehnungen. Deutsche Zeitschrift für Sportmedizin, 50(1), 10-16.

Ylinen, J., Kankainen, T., Kautiainen, H., Rezasoltani, A., Kuukkanen, T., \& Häkkinen, A. (2009). Effect of stretching on hamstring muscle compliance. Journal of Rehabilitation Medicine, 41(1), 80-84.

Youdas, J.W., Garrett, T.R., Suman, V.J., Bogard, C.L., Hallman, H.O., \& Carey, J.R. (1992). Normal range of motion of the cervical spine. An initial goniometric study. Physical Therapy, 72(11), 770-780.

Young, P., Ferguson, C., Bañuelos, S., \& Gautel, M. (1998). Molecular structure of the sarcomeric Z-disk. Two types of titin interactions lead to an asymmetrical sorting of alpha-actinin. The EMBO Journal, 17(6), 1614-1624.

Young, R., Nix, S., Wholohan, A., Bradhurst, R., \& Reed, L. (2013). Interventions for increasing ankle joint dorsiflexion. A systematic review and meta-analysis. Journal of Foot and Ankle Research, 6(1), 46. doi: 10.1186/1757-1146-6-46

Young, W.B. (2007). The use of static stretching in warm-up for training and competition. International Journal of Sports Physiology and Performance, 2(2), 212-216.

Young, W.B., \& Behm, D.G. (2002). Should static stretching be used during a warm-up for strength and power activities? Strength and Conditioning Journal, 24(6), 33-37.

Yuktasir, B., \& Kaya, F. (2009). Investigation into the long-term effects of static and PNF stretching exercises on range of motion and jump performance. Journal of Bodywork and Movement Therapies, 13(1), 11-21. 
Anhang 


\section{MatLab-Code zur Berechnung der maximalen Beweglichkeit}

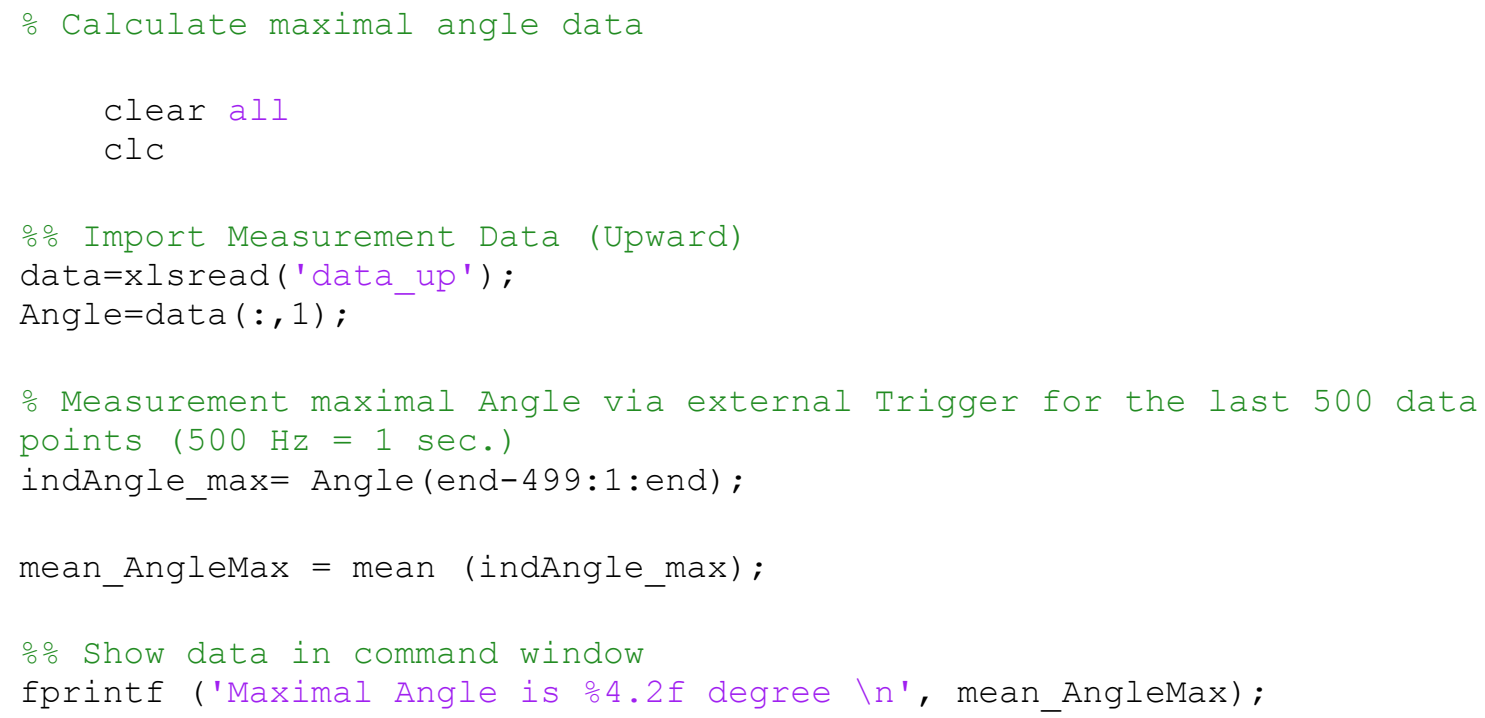




\section{MatLab-Code zur Berechnung der maximalen Dehnungsspannung}

o Calculate gravity-correction data

- Calculate maximal angle and maximal torque data

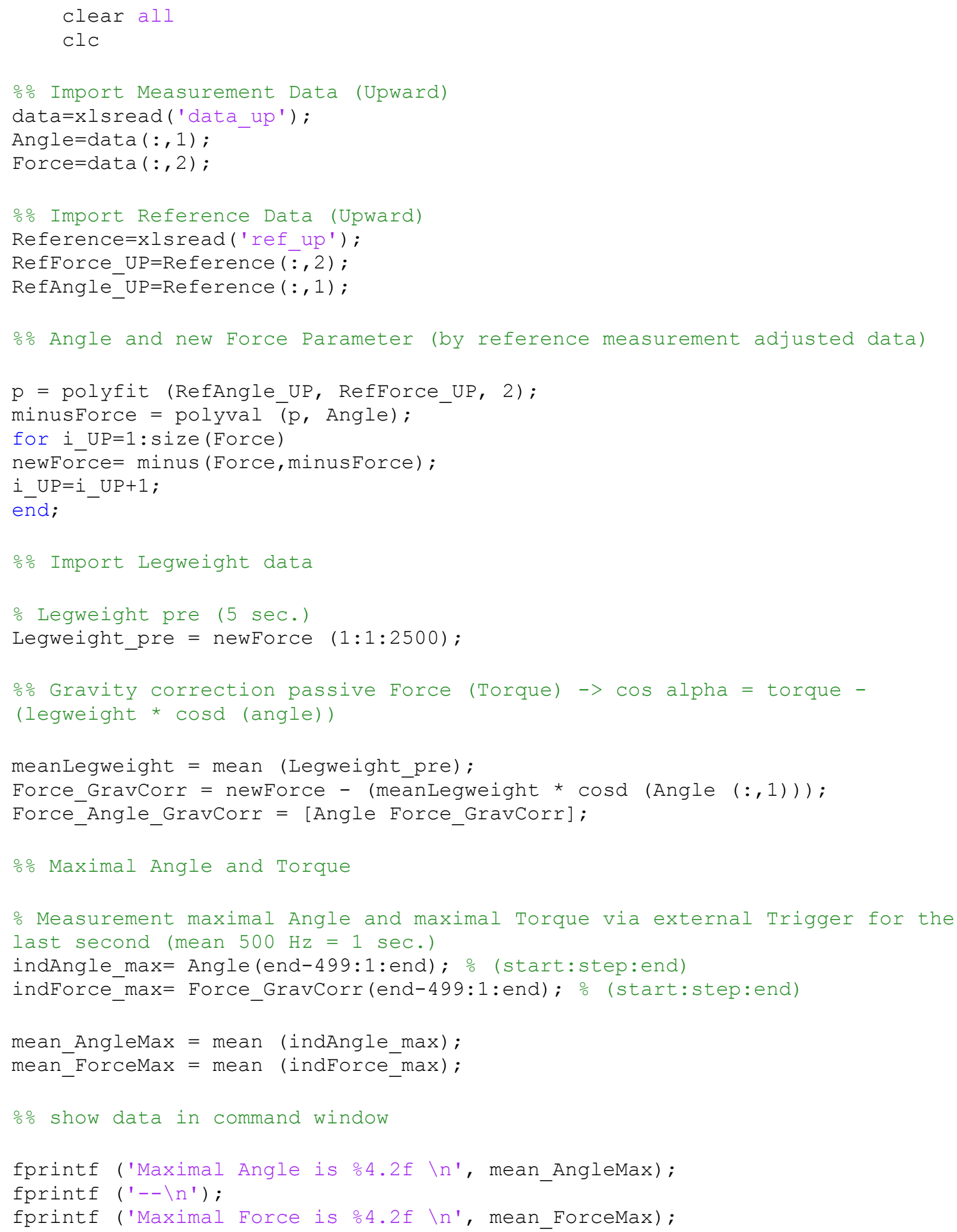




\section{MatLab-Code zur Berechnung der passiven-elastischen Stiffness}

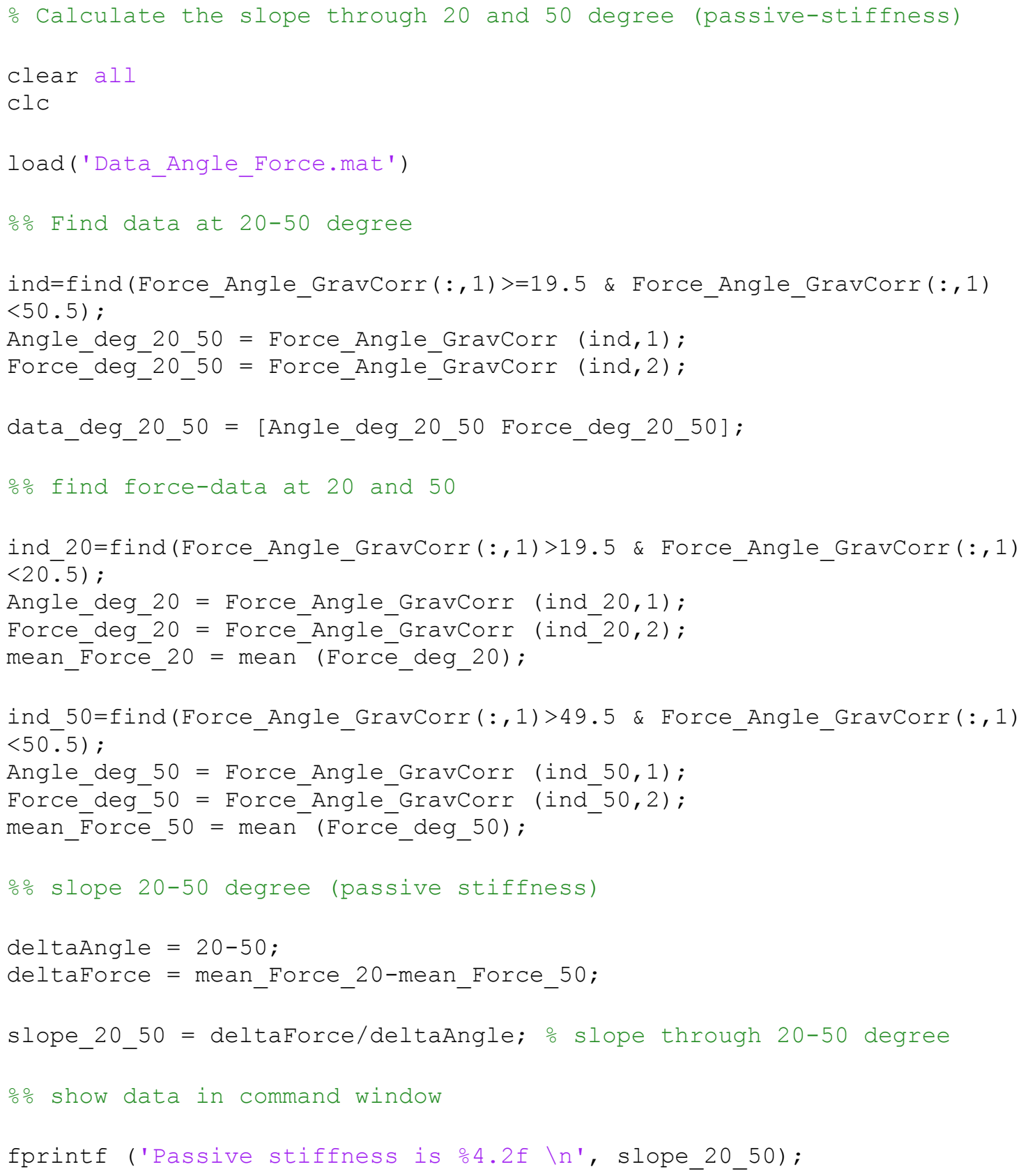




\section{MatLab-Code zur Berechnung der passiven-elastischen Energie}

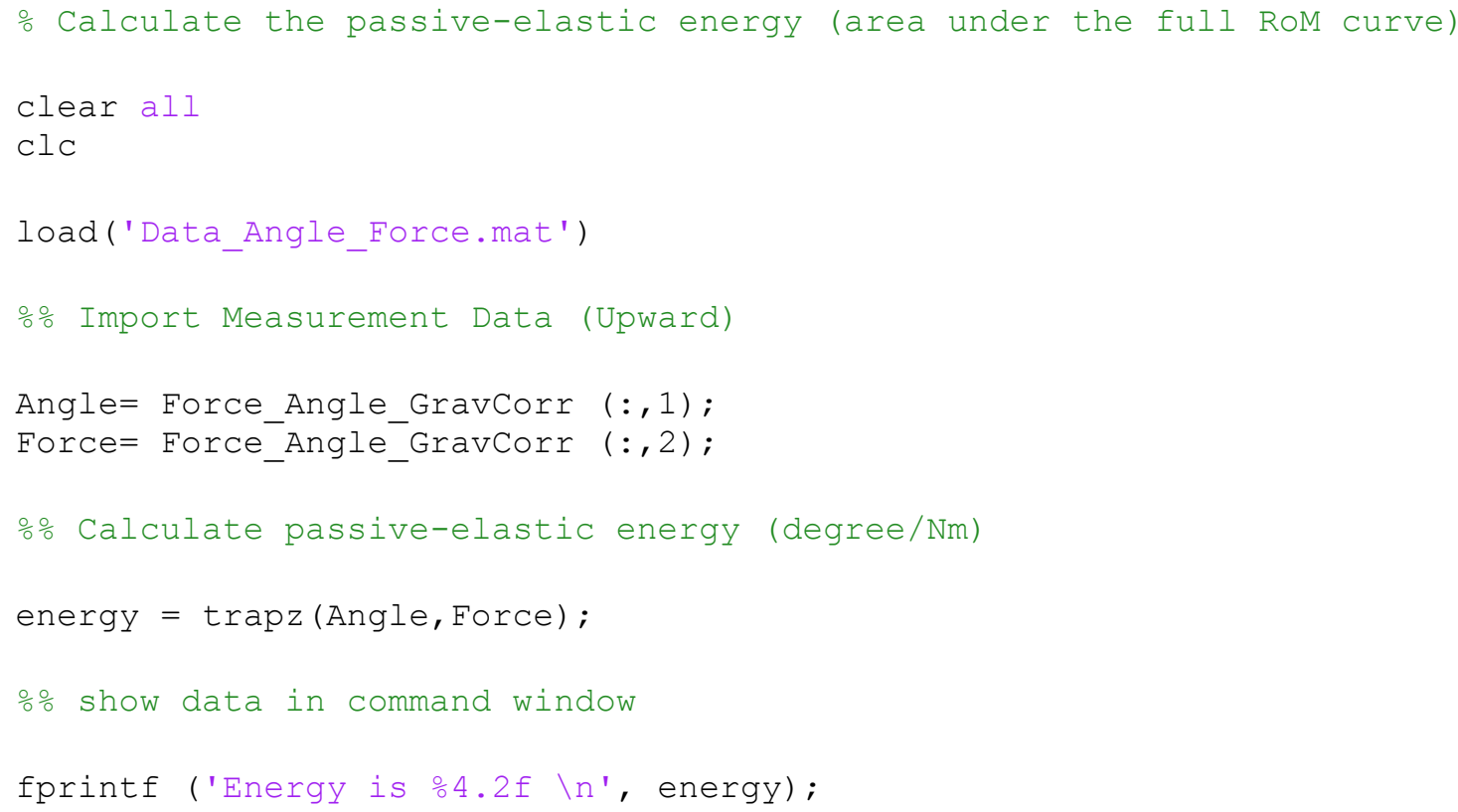




\section{MatLab-Code zur Berechnung der EMG-Parameter}

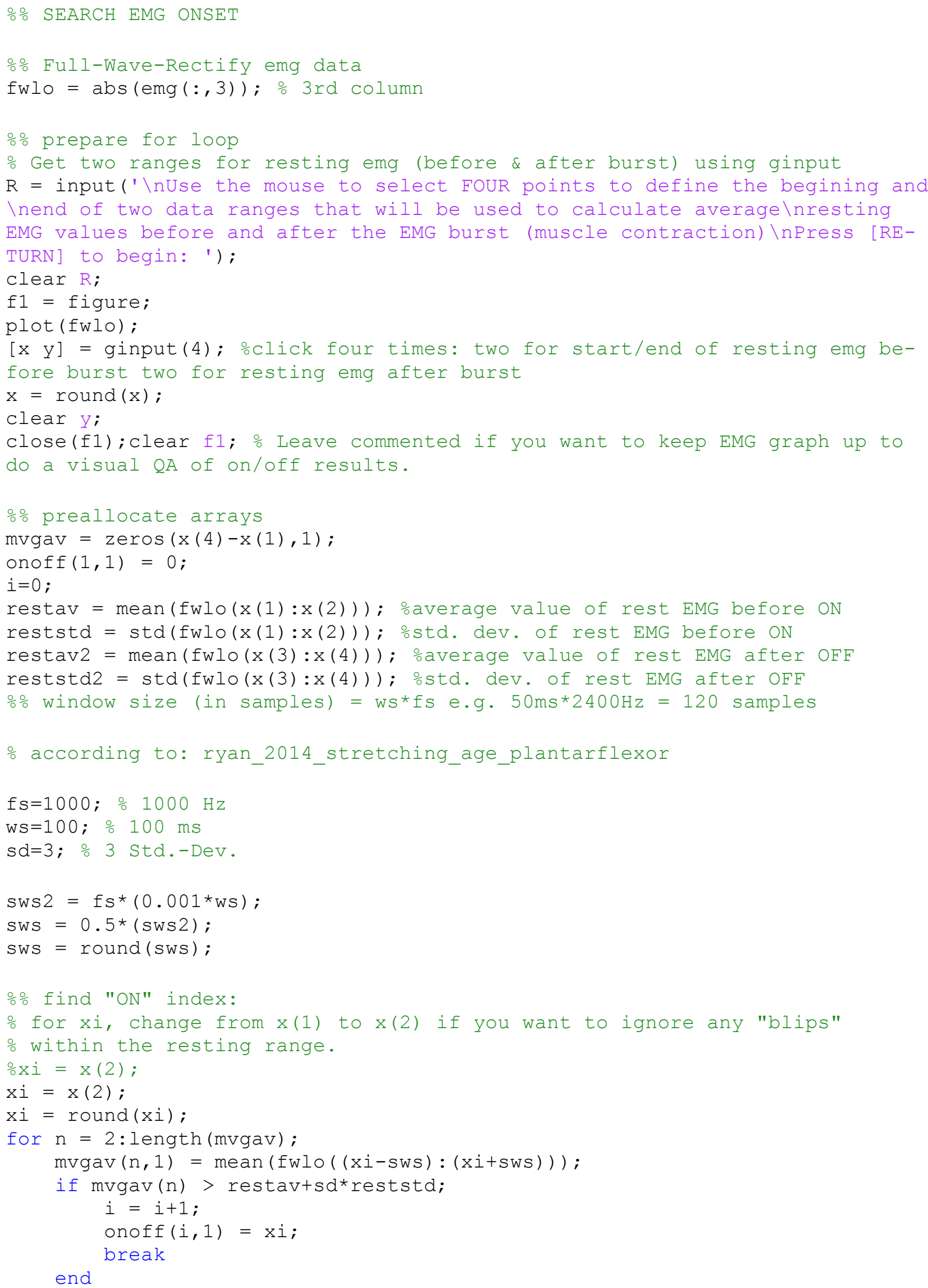


end

$$
x i=x i+1
$$

- Data summary

emg_on angle $=\operatorname{emg}(x i, 1)$;

emg_on_torque $=\operatorname{emg}(\mathrm{xi}, 2)$;

응 EMG-Onset percentage from ROMmax.

load ('Data_Angle_Force.mat') ;

p_emg $=(100 /$ mean_AngleMax $) *$ emg_on_angle;

응 show data in command window

fprintf ('Angle at EMG onset is \%4.2f degree \n', emg_on_angle); fprintf ('-- \n');

fprintf ('Percentage EMG onset from ROM max. is o4.2f \n', p_emg); 